\title{
Ascaroside Signaling in the Bacterivorous Nematode Caenorhabditis remanei Encodes the Growth Phase of its Bacterial Food Source
}

Franziska Dolke, ${ }^{1}$ Chuanfu Dong, ${ }^{1}$ Siva Bandi, ${ }^{2}$ Christian Paetz, ${ }^{3}$ Gaétan Glauser,${ }^{4}$ and Stephan H. von Reuss ${ }^{* 1,2,4}$

1: Department of Bioorganic Chemistry, Max Planck Institute for Chemical Ecology, Hans-Knöll Straße 8, D-07745 Jena, Germany. 2: Laboratory for Bioanalytical Chemistry, Institute of Chemistry, University of Neuchâtel, Avenue de Bellevaux 51, CH-2000 Neuchâtel, Switzerland.

3: Research Group Biosynthesis / NMR, Max Planck Institute for Chemical Ecology, Hans-Knöll Straße 8, D-07745 Jena, Germany. 4: Neuchatel Platform for Analytical Chemistry (NPAC), University of Neuchâtel, Avenue de Bellevaux 51, CH-2000 Neuchâtel, Switzerland.

*stephan.vonreuss@unine.ch

SUPPORTING INFORMATION

Pages

Experimental procedures

$\mathrm{S} 2-\mathrm{S} 19$

Supporting Tables S1 - S6

$S 20-S 26$

Supporting Figures S1 - S21

$S 27-S 80$

Supporting NMR spectra (Figures S22 - S124)

$\mathrm{S} 83-\mathrm{S} 198$

References

S199 


\section{EXPERIMENTAL PROCEDURES}

Organisms. Escherichia coli OP50 was obtained from the Caenorhabditis Genetics Center (CGC) at the University of Minnesota. The E. coli $\Delta c f a:: k a n$ knockout mutant strain JW1653-1 from the Keio collection ${ }^{[1]}$ was obtained from the E. coli Genetic Stock Center (CGSC) at Yale University. Caenorhabditis remanei PB4641 (USA), C. remanei PB228 (USA), C. remanei SB146 (Germany), C. remanei JU1082 (Japan), C. latens JU724 (China), C. latens VX88 (China), C. elegans N2 (UK), C. elegans AB1 (Australia), C. nigoni JU1422 (India), C. briggsae AF16 (India), C. sinica JU727 (China), C. tropicalis JU1373 (La Réunion), C. wallacei JU1904 (Indonesia), C. doughertyi JU1771 (India), C. brenneri PB2801 (Costa Rica), C. japonica DF5081 (Japan), C. afra JU1286 (Ghana), C. portoensis EG4788 (Portugal), C. virilis JU1968 (France), and C. monodelphis JU1667 (Germany) were obtained from the Caenorhabditis Genetics Center (CGC). C. remanei wildtype isolates MY726 (Germany) and MY2231 (Germany) were kindly provided by Prof. Hinrich Schulenburg (CAU Kiel, Germany).

Cultivation of Escherichia coli. E. coli OP50 and E. coli $\Delta$ cfa were cultivated on Lysogeny broth (LB) agar at $37^{\circ} \mathrm{C}$. Liquid cultures were grown overnight $(12-14 \mathrm{~h})$ in liquid LB medium at $37^{\circ} \mathrm{C}$ and $200 \mathrm{rpm}$. Bacterial cells were harvested by centrifugation at $5000 \mathrm{~g}$ for $10 \mathrm{~min}$, washed with M9 buffer, and stored at $4{ }^{\circ} \mathrm{C}$ until used as food for nematode cultures.

Preparation of Caenorhabditis exometabolome extracts for comprehensive ascaroside screening. Wild-type isolates of fifteen Caenorhabditis species (Figure S12) including C. remanei PB4641, C. latens VX88, C. elegans N2 and C. elegans AB1, C. nigoni JU1422, C. briggsae AF16, C. sinica JU727, C. tropicalis JU1373, C. wallacei JU1904, C. doughertyi JU1771, C. brenneri PB2801, C. japonica DF5081, C. afra JU1286, C. portoensis EG4788, C. virilis JU1968, and C. monodelphis JU1667 were cultivated at $23^{\circ} \mathrm{C}$ on NGM agar seeded with E. coli OP50. Mixed stage nematodes from five $10 \mathrm{~cm}$ plates collected in M9 buffer served as inoculums for liquid cultures grown in $100 \mathrm{~mL} \mathrm{~S}$-medium at $23^{\circ} \mathrm{C}$ and $150 \mathrm{rpm}$. Concentrated E. coli OP50 bacteria pellet from an overnight culture in LB medium at $37^{\circ} \mathrm{C}$ and $200 \mathrm{rpm}$ was provided as food from days 1-7, after which the cultures were starved for 7 days. After 14 days, nematodes were separated by centrifugation $\left(5 \mathrm{~min}\right.$ at $5000 \mathrm{~g}$ ), frozen at $-80^{\circ} \mathrm{C}$ and lyophilized. The filtered supernatant representing the exometabolome was frozen at $-80^{\circ} \mathrm{C}$, lyophilized, and extracted with $3 \times 100 \mathrm{~mL}$ methanol for $12 \mathrm{~h}$ each. The combined extract was filtered, concentrated to dryness under reduced pressure at $40{ }^{\circ} \mathrm{C}$, and reconstituted in $1 \mathrm{~mL}$ methanol. Aliquots were analyzed by HPLC-ESI-HR-MS, HPLC-ESI-HR-MS/MS, HPLC-MS/MS precursor ion scanning for $m / z$ 73.0, and HPLC-ESI-(-)-MS ${ }^{n}$.

Quantification of fatty acid ascarosides in exometabolome extracts of Caenorhabditis remanei and Caenorhabditis latens. Five wild-type isolates of Caenorhabditis remanei including the reference strain PB4641 (USA) as well as PB228 (USA), JU1082 (Japan), SB146 (Germany), MY726 (Germany), and MY2231 (Germany) and two wild-type isolates of Caenorhabditis latens including the reference strain VX88 (China) as well as JU724 (China) (previously described as C. remanei ${ }^{[2]}$ but now identified as $C$. latens) $)^{[3]}$ were cultivated at $23^{\circ} \mathrm{C}$ on $\mathrm{NGM}$ agar seeded with E. coli OP50. Mixed stage nematodes from five $10 \mathrm{~cm}$ plates collected in $\mathrm{M} 9$ buffer served as inoculums for liquid cultures grown in $100 \mathrm{~mL} \mathrm{~S}$-medium at $23^{\circ} \mathrm{C}$ and $150 \mathrm{rpm}$. Concentrated 
E. coli OP50 bacteria pellet from an overnight culture in LB medium at $37^{\circ} \mathrm{C}$ and $200 \mathrm{rpm}$ was provided as food from days 1-7, after which the cultures were starved for 7 days. After 14 days, nematodes were separated by centrifugation $\left(5 \mathrm{~min}\right.$ at $5000 \mathrm{~g}$ ), frozen at $-80^{\circ} \mathrm{C}$ and lyophilized. The filtered supernatant representing the exometabolome was frozen at $-80{ }^{\circ} \mathrm{C}$, lyophilized, and extracted with $3 \times 100 \mathrm{~mL}$ methanol for $12 \mathrm{~h}$ each. The combined extract was filtered, concentrated to dryness under reduced pressure at $40{ }^{\circ} \mathrm{C}$, and reconstituted in $1 \mathrm{~mL}$ methanol. Aliquots were analyzed by HPLC-ESI-HR-MS, HPLC-ESI-HR-MS/MS, HPLC-MS/MS precursor ion scanning for $m / z$ 73.0, and HPLC-ESI-(-)-MS ${ }^{n}$. Quantification of fatty acid ascarosides was performed by integration of signals for $[\mathrm{M}-\mathrm{H}]^{-}$in extracted ion chromatograms from HPLC-ESI$(-)-H R-M S$ analysis. For each strain three biological replicates were analyzed and data shown in Figure S11 represent the calculated mean \pm SEM.

Isolation of fatty acid ascarosides from the Caenorhabditis remanei exometabolome extract. Fatty acid ascaroside asc-C4-cyC11 (fasc\#1, 3) was isolated from $1.5 \mathrm{~L}$ of a C. remanei PB4641 liquid culture grown at $23^{\circ} \mathrm{C}$ for 14 days as described for the small-scale cultures. The filtered culture supernatant was frozen at $-80^{\circ} \mathrm{C}$, lyophilized, and the powdered residue extracted with $3 \times 200 \mathrm{ml}$ methanol for $12 \mathrm{~h}$ each. The filtered extract was concentrated to dryness under reduced pressure at $40{ }^{\circ} \mathrm{C}$ to afford the exometabolome extract. Fatty acid ascaroside asc-C4$\mathrm{C} 12: 1$ (fasc\#2, 4), asc-C4-C10:1 (fasc\#3, 5), as well as the diversity of additional minor fatty acid ascaroside components (Figure S6) were enriched from the combined exometabolome extracts of various C. remanei isolates (PB4641, PB228, JU1082, SB146, MY726, MY2231) corresponding to a total amount of $1.6 \mathrm{~L}$ of liquid culture medium. The exometabolome extracts were adsorbed onto $2 \mathrm{~g}$ of celite and fractionated by solid phase extraction on $5 \mathrm{~g} \mathrm{RP}-\mathrm{C} 18$ (Chromabond, Macherey-Nagel) using increasing concentrations of methanol in water as eluent to afford 10 fractions ( 0 to $100 \%$, in $10 \%$ steps, $10 \mathrm{ml}$ each) that were concentrated to dryness under reduced pressure. Aliquots of $10 \mu \mathrm{l}$ were analyzed by HPLC-ESI-(-)-HR-MS and fractions shown to contain target components (eluted with $70-80 \%$ methanol) were subsequently submitted to semi-preparative HPLC using an Agilent HP-1100 HPLC instrument equipped with a Grom-Sil 120 ODS-4 HE column $(250 \times 8 \mathrm{~mm}, 5 \mu \mathrm{m})$ coupled to a Gilson 206 Abimed fraction collector. A flow rate of $2 \mathrm{ml} / \mathrm{min}$ with gradient elution was used, starting at $3 \%$ acetonitrile in $0.5 \%$ aqueous acetic acid for 3 minutes, followed by a linear increase to $100 \%$ acetonitrile with $0.5 \%$ acetic acid within 30 minutes. Aliquots of $10 \mu \mathrm{l}$ were analyzed by HPLC-ESI-(-)-HR-MS as described before. Fractions containing the fatty acid ascaroside target compounds were concentrated under reduced pressure, dried in high vacuum overnight, and analyzed by HPLC-ESI-HR-MS/MS (Table S6), HPLC-ESI-(-)-MS ${ }^{n}$ (Table S5, Figure S9), and NMR spectroscopy (Table S1).

(3'R,3S,4S,7S)-7-[(3'-[(3',,6''-Dideoxy-a-L-arabino-hexopyranosyl)-oxy]-butanoyl)oxy]-3,4cis-methylenedecanoic acid (fasc\#1, asc-C4-cyC11 (3)) isolated as a yellowish oil from the C. remanei PB4641 exometabolome $(\sim 100 \mu \mathrm{g})$, for ${ }^{1} \mathrm{H}$ and ${ }^{13} \mathrm{C}$ NMR data see Table S1; HR-MS obsd. $m / z 415.2343[\mathrm{M}-\mathrm{H}]^{-}$, calcd. 415.2337 for $\mathrm{C}_{21} \mathrm{H}_{35} \mathrm{O}_{8}, \Delta=1.5 \mathrm{ppm}$.

(3Z)-(3'R)-7-[(3'-[(3', 6"'-Dideoxy-a-L-arabino-hexopyranosyl)-oxy]-butanoyl)oxy]-dodec-3enoic acid (fasc\#2, asc-C4-C12:1 (4)) isolated as a yellowish oil from pooled exometabolome extracts of various $C$. remanei strains $(\sim 40 \mu \mathrm{g})$, for ${ }^{1} \mathrm{H}$ and ${ }^{13} \mathrm{C}$ NMR data see Table S1; HR-MS obsd. $m / z 429.2504[\mathrm{M}-\mathrm{H}]^{-}$, calcd. 429.2494 for $\mathrm{C}_{22} \mathrm{H}_{37} \mathrm{O}_{8}, \Delta=2.5 \mathrm{ppm}$. 
(3Z)-(3'R)-7-[(3'-[(3", 6"'-Dideoxy-a-L-arabino-hexopyranosyl)-oxy]-butanoyl)oxy]-dec-3enoic acid (fasc\#3, asc-C4-C10:1 (5)) partially enriched as a yellowish oil from pooled exometabolome extracts of various $C$. remanei strains ( $\sim \mathrm{\mu g})$, HR-MS obsd. $\mathrm{m} / \mathrm{z} 401.2181$ [M $\mathrm{H}^{-}$, calcd. $\mathrm{m} / \mathrm{z} 401.2181$ for $\mathrm{C}_{20} \mathrm{H}_{33} \mathrm{O}_{8}, \Delta=0.1 \mathrm{ppm}$.

Isolation of fatty acid ascarosides from the Caenorhabditis latens exometabolome extract. Fatty acid ascarosides were isolated from $1.2 \mathrm{~L}$ liquid culture medium of the C. Iatens (C. sp. n23) reference strain $\mathrm{VX} 88^{[3]}$ grown at $23^{\circ} \mathrm{C}$ for 14 days. The filtered culture supernatant was frozen at $-80^{\circ} \mathrm{C}$, lyophilized, and the powdered residue extracted with $3 \times 200 \mathrm{ml}$ methanol for $12 \mathrm{~h}$ each. The filtered extract was concentrated to dryness under reduced pressure and the resulting exometabolome extract was adsorbed onto $2 \mathrm{~g}$ of celite and fractionated by solid phase extraction on $5 \mathrm{~g} \mathrm{RP}-\mathrm{C} 18$ (Chromabond, Macherey-Nagel) using increasing concentrations of methanol in water as eluent to afford 10 fractions ( 0 to $100 \%$, in $10 \%$ steps, $10 \mathrm{ml}$ each) that were concentrated to dryness under reduced pressure. Aliquots of $10 \mu \mathrm{l}$ were analyzed by HPLC-ESI(-)-HR-MS and fractions shown to contain target components were subsequently submitted to semi-preparative HPLC using an Agilent HP-1100 HPLC instrument equipped with a Grom-Sil 120 ODS-4 HE column $(250 \times 8 \mathrm{~mm}, 5 \mu \mathrm{m})$ coupled to a Gilson 206 Abimed fraction collector. A flow rate of $2 \mathrm{ml} / \mathrm{min}$ with gradient elution was used, starting at $3 \%$ acetonitrile in $0.5 \%$ aqueous acetic acid for 3 minutes, followed by a linear increase to $100 \%$ acetonitrile with $0.5 \%$ acetic acid within 30 minutes. Aliquots of $10 \mu \mathrm{l}$ were analyzed by HPLC-ESI-(-)-HR-MS as described before. Fractions containing the fatty acid ascaroside target compounds were concentrated under reduced pressure, dried in high vacuum overnight, and analyzed by HPLC-ESI-HR-MS/MS (Table S6), HPLC-ESI-(-)-MSn (Table S5, Figure S9), and NMR spectroscopy (Table S4).

7-[(3'-[(3",,6"'-Dideoxy-a-L-arabino-hexopyranosyl)-oxy]-butanoyl)oxy]-octanoic acid (fasc\#4, asc-C4-C8 (7)) isolated as a yellowish oil from the C. latens VX88 exometabolome ( 40 $\mu \mathrm{g}$ ), for ${ }^{1} \mathrm{H}$ and ${ }^{13} \mathrm{C}$ NMR data see Table S4; HR-MS obsd. $\mathrm{m} / \mathrm{z} 375.2031$ [M - H] ${ }^{-}$, calcd. 375.2024 for $\mathrm{C}_{18} \mathrm{H}_{31} \mathrm{O}_{8}, \Delta=1.9 \mathrm{ppm}$.

7-[(3'-[(3",6'"-Dideoxy-a-L-arabino-hexopyranosyl)-oxy]-butanoyl)oxy]-hexanoic acid (fasc\#5, asc-C4-C6) isolated as a yellowish oil from the $C$. latens VX88 exometabolome $(\sim 15 \mu \mathrm{g})$, for ${ }^{1} \mathrm{H}$ and ${ }^{13} \mathrm{C}$ NMR data see Table S4; HR-MS obsd. $\mathrm{m} / \mathrm{z} 347.1717$ [M - H] $]^{-}$, calcd. 347.1711 for $\mathrm{C}_{16} \mathrm{H}_{27} \mathrm{O}_{8}, \Delta=1.7 \mathrm{ppm}$.

Preparation of methyl 7-hydroxy-cis-3,4-methylenedecanoate (9) from asc-C4-cyC11 (3). A solution of $\sim 75 \mu \mathrm{g}$ asc-C4-cyC11 (fasc\#1, 3, $180 \mathrm{nmol}$ ) from C. remanei PB4641 in $500 \mu \mathrm{L} \mathrm{CD}_{3} \mathrm{OD}$ was treated with $5 \mu \mathrm{l} 40 \% \mathrm{NaOD}$ in $\mathrm{D}_{2} \mathrm{O}(50 \mu \mathrm{mol}) .{ }^{1} \mathrm{H}$ NMR spectra were recorded over several days until hydrolysis of the ester bond was complete. The solution was acidified with $10 \mu \mathrm{l}$ acetic acid and concentrated to dryness under reduced pressure at $40{ }^{\circ} \mathrm{C}$ to afford a mixture of 7 hydroxy-cis-3,4-methylenedecanoic acid (8) and asc-C4 (19). The residue was dissolved in $1 \mathrm{ml}$ of a methanol / toluene mixture $(1: 1, \mathrm{v} / \mathrm{v})$ and treated with $90 \mu \mathrm{l} 2 \mathrm{M}$ (trimethylsilyl) diazomethane in diethyl ether $(180 \mu \mathrm{mol})$. After stirring for $1 \mathrm{~h}$, excess reagent was quenched by addition of 30 $\mu \mathrm{l}$ acetic acid $(525 \mu \mathrm{mol})$. The solution was concentrated under reduced pressure and the residue chromatographed on silica gel using a stepwise gradient of ethyl acetate in hexane (0 to $100 \%$ in $10 \%$ steps). Using GC-EIMS analysis the product was traced to the $30 \%$ ethyl acetate fraction, 
which was concentrated to dryness under reduced pressure to afford approximately $20 \mu \mathrm{g}$ methyl 7-hydroxy-cis-3,4-methylenedecanoate $(9,90 \mathrm{nmol}, 50 \%$ yield over two steps) as a yellowish oil.

Methyl (3S,4S,7S)-7-hydroxy-3,4-cis-methylenedecanoic acid (9). $\left.{ }^{1} \mathrm{H} \mathrm{NMR} \mathrm{(400} \mathrm{MHz,} \mathrm{CDCl}_{3}\right)$ ס: $-0.13(1 \mathrm{H}, \mathrm{m}), 0.74(1 \mathrm{H}, \mathrm{m}), 0.83(1 \mathrm{H}, \mathrm{m}) 0.78-0.90(2 \mathrm{H}, \mathrm{m}), 0.93(3 \mathrm{H}, \mathrm{t}, \mathrm{J}=7.1 \mathrm{~Hz}), 1.12(1 \mathrm{H}$, $\mathrm{m}), 1.38(2 \mathrm{H}, \mathrm{m}), 1.42(2 \mathrm{H}, \mathrm{m}), 1.54(2 \mathrm{H}, \mathrm{m}), 2.34(2 \mathrm{H}, \mathrm{dd}, \mathrm{J}=7.5 \mathrm{~Hz}, \mathrm{~J}=3.0 \mathrm{~Hz}), 3.63(1 \mathrm{H}, \mathrm{m})$, $3.70(3 \mathrm{H}, \mathrm{s})$; HR-MS obsd. $\mathrm{m} / \mathrm{z} 237.1468[\mathrm{M}+\mathrm{Na}]^{+}$, calcd. $m / z 237.1461$ for $\mathrm{C}_{12} \mathrm{H}_{23} \mathrm{NaO}_{3}, \Delta=3.0$ ppm.

Comparative NMR analysis of diastereoisomeric MTPA esters ((R)-MTPA-10 \& (S)-MTPA10). A solution of $\sim 10 \mu \mathrm{g}$ methyl (7S)-hydroxy-cis-3,4-methylene decanoate (9) in $30 \mu \mathrm{l}$ chloroform- $\mathrm{d}_{1}$ was treated with $2.1 \mu \mathrm{l}$ pyridine- $\mathrm{d}_{5}$ ("100\%" D) and $0.5 \mu \mathrm{l}(S)$ - or $(R)$-MTPA chloride in $5 \mu \mathrm{l} \mathrm{chloroform}-\mathrm{d}_{1}$ (ratio of alcohol / MTPA chloride / pyridine $=\mathrm{ca} .1 / 50 / 500$ ) to afford the corresponding $(R)$ - or $(S)$-MTPA esters (10), respectively. After $1 \mathrm{~h}$ at room temperature the ${ }^{1} \mathrm{H}$ NMR, dqf-COSY and HSQC spectra were recorded. In addition, 1D-selective TOCSY spectra (SELTOCSY) were recorded using irradiation of the terminal methyl protons with 80 ms mixing time, the acyloxymethine protons with $30 \mathrm{~ms}$ mixing time, or the cis-configurated cyclopropyl methylene protons with 30 and 60 ms mixing times. Data were analyzed using the Topspin 3.2 (Bruker Biospin) and MNova 9.0 (Mestrelab Research) software and $\Delta \delta^{S R}\left(=\delta_{S}-\delta_{R}\right)$ values were calculated (Table S2).

Palladium-catalyzed hydrogenation of fatty acid ascarosides. C. remanei PB4641 exometabolome fractions enriched with fatty acid ascarosides (fasc) in $20 \mu \mathrm{l}$ methanol (obtained by RP-C18-solid phase extraction and HPLC fractionation of the culture supernatant extract as described) were diluted with $500 \mu \mathrm{l}$ methanol, treated with $10 \mathrm{mg} \mathrm{Pd} / \mathrm{C}(10 \%, \mathrm{w} / \mathrm{w})$ and hydrogen gas bubbled through the vigorously stirred mixture. After $3 \mathrm{~h}$ the mixtures were filtered over cotton, concentrated under reduced pressure, and the residues taken up in $20 \mu \mathrm{l}$ methanol. Comparative HPLC-ESI-(-)-HR-MS/MS and HPLC-ESI-(-)-MS ${ }^{n}$ analysis using the untreated exometabolome fraction as control facilitated the discrimination of a fatty acid ascaroside series carrying inert cyclopropyl fatty acid residues from those with unsaturated fatty acid residues (Figure S7).

Palladium-catalyzed deuterogenation of asc-C4-C12:1 (4). To confirm the assignment of fragment ion signals observed upon multiple stage mass spectrometry $\left(\mathrm{MS}^{n}\right)$ of fatty acid ascarosides (Figure S9), the 3,4-dideutero-derivative of asc-C4-C12 was prepared by $\mathrm{Pd} / \mathrm{C}$ catalyzed deuterogenation of asc-C4-C12:1 (fasc\#2, 4) the structure of which was identified by NMR spectroscopy (Table S1). A fraction enriched in asc-C4-C12:1 (fasc\#2, 4) in $10 \mu \mathrm{l}$ methanol (obtained by RP-C18-solid phase extraction and HPLC fractionation of the C. remanei culture supernatant extract as described) was diluted with $500 \mu \mathrm{l}$ methanol, treated with $10 \mathrm{mg} \mathrm{Pd} / \mathrm{C}$ $(10 \%, w / w)$ and deuterium gas bubbled through the vigorously stirred mixture. After $3 \mathrm{~h}$ the mixture was filtered over cotton, concentrated under reduced pressure, and the residue taken up in $10 \mu \mathrm{l}$ methanol for HPLC-ESI-(-)-HR-MS/MS and HPLC-ESI-(-)-MS ${ }^{n}$ analysis. Comparative $\mathrm{MS}^{n}$ analysis of asc-C4-C12 and [3,4-D $]$-asc-C4-C12, obtained by hydrogenation and deuterogenation of asc-C4-C12:1 (fasc\#2, 4), respectively, facilitated the assignment of fragment ion signals (Figure S8). 
(3'R)-7-[(3'-[(3', 6''-Dideoxy- $\alpha$-L-arabino-hexopyranosyl)-oxy]-butanoyl)oxy]-3,4-dideuterododecanoic acid ([3,4- $\left.\mathrm{D}_{2}\right]$-asc-C4-C12, $\left[\mathrm{D}_{2}\right]$-fasc\#9) by deuterogenation of asc-C4-C12:1 (fasc\#2, 4) from C. remanei PB4641: HPLC-ESI-(-)-HR-MS/MS: obsd. m/z 433.2609 [M - H] ${ }^{-}$, calcd. $\mathrm{m} / \mathrm{z} 433.2619$ for $\mathrm{C}_{22} \mathrm{H}_{37} \mathrm{D}_{2} \mathrm{O}_{8} ; \Delta=3.7 \mathrm{ppm}$; obsd. $\mathrm{m} / \mathrm{z} 285.2041$ [M $\left.-\mathrm{C}_{6} \mathrm{H}_{13} \mathrm{O}_{4}\right]^{-}$, calcd. $\mathrm{m} / \mathrm{z}$ 285.2040 for $\mathrm{C}_{16} \mathrm{H}_{25} \mathrm{D}_{2} \mathrm{O}_{6} ; \Delta=-0.1 \mathrm{ppm}$; for HPLC-MS : see Figure S8.

(3'R)-7-[(3'-[(3',,6''-Dideoxy-a-L-arabino-hexopyranosyl)-oxy]-butanoyl)oxy]-dodecanoic acid (asc-C4-C12, fasc\#9) by hydrogenation of asc-C4-C12:1 (fasc\#2, 4) from C. remanei PB4641: HPLC-ESI-(-)-HR-MS/MS: obsd. $\mathrm{m} / \mathrm{z} 431.2651$ [M - H] $]^{-}$, calcd. $\mathrm{m} / \mathrm{z} 431.2650$ for $\mathrm{C}_{22} \mathrm{H}_{39} \mathrm{O}_{8} ; \Delta=-0.1 \mathrm{ppm}$; obsd. $\mathrm{m} / \mathrm{z} 285.1913\left[\mathrm{M}-\mathrm{C}_{6} \mathrm{H}_{13} \mathrm{O}_{4}\right]^{-}$, calcd. $\mathrm{m} / \mathrm{z} 283.1915$ for $\mathrm{C}_{16} \mathrm{H}_{27} \mathrm{O}_{6} ; \Delta$ $=0.6$ ppm; for HPLC-MS ${ }^{n}$ : see Figure S8.

Osmium tetroxide mediated cis-1,2-dihydroxylation of fatty acid ascarosides. C. remanei exometabolome fractions enriched in fatty acid ascarosides in $10 \mu \mathrm{l}$ methanol (obtained by RPC18-solid phase extraction and HPLC fractionation of the culture supernatant extract as described) were diluted with $500 \mu \mathrm{l}$ methanol and treated with $10 \mu \mathrm{l}$ osmium tetroxide solution $(2.5 \%, w / v)$ in tert-butanol. After stirring for $3 \mathrm{~h}$ the mixtures were treated with $10 \mathrm{mg} \mathrm{Na}_{2} \mathrm{~S}_{2} \mathrm{O}_{3}$ and $50 \mu \mathrm{L}$ water and stirred for $1 \mathrm{~h}$. The mixtures were concentrated under reduced pressure and the residues dissolved in $200 \mu \mathrm{L}$ water and absorbed on a preconditioned solid phase extraction column (50 mg C18-hydra, Chromabond, Macherey-Nagel). The columns were washed with $1 \mathrm{ml}$ water and products were eluted with $1 \mathrm{ml}$ methanol, concentrated under reduced pressure, and the residues taken up in $10 \mu \mathrm{l}$ methanol for HPLC-ESI-(-)-HR-MS/MS and HPLC-ESI-(-)-MS ${ }^{n}$ analysis (Figure S10).

7-[(3'-[(3',,6"'-Dideoxy-a-L-arabino-hexopyranosyl)-oxy]-butanoyl)oxy]-3,4-dihydroxydecanoic acid (asc-C4-3,4-(OH) ${ }_{2}-\mathrm{C} 10$ ) from $\mathrm{OsO}_{4}$ dihydroxylation of asc-C4-C10:1 (fasc\#3, 5) from C. remanei PB4641: HPLC-ESI-(-)-HR-MS/MS: obsd. $\mathrm{m} / \mathrm{z} 435.2243[\mathrm{M}-\mathrm{H}]^{-}$, calcd. $\mathrm{m} / \mathrm{z}$ 435.2236 for $\mathrm{C}_{20} \mathrm{H}_{35} \mathrm{O}_{10} ; \Delta=-1.6 \mathrm{ppm}$; obsd; $\mathrm{m} / \mathrm{z} 287.1503$ [M $\left.-\mathrm{C}_{6} \mathrm{H}_{13} \mathrm{O}_{4}\right]^{-}$, calcd. $\mathrm{m} / \mathrm{z} 287.1500$

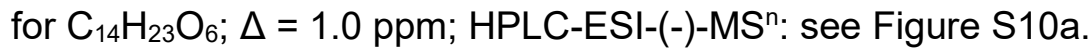

7-[(3'-[(3','6"'-Dideoxy-a-L-arabino-hexopyranosyl)-oxy]-butanoyl)oxy]-3,4-dihydroxydodecanoic acid (asc-C4-3,4-(OH) $)_{2}-\mathrm{C12}$ ) from $\mathrm{OsO}_{4}$ dihydroxylation of asc-C4-C12:1 (fasc\#2, 4) from C. remanei PB4641: HPLC-ESI-(-)-HR-MS/MS: obsd. m/z 463.2552 [M - H] ${ }^{-}$, calcd. $\mathrm{m} / \mathrm{z} 463.2549$ for $\mathrm{C}_{22} \mathrm{H}_{39} \mathrm{O}_{10} ; \Delta=-0.8$ ppm; obsd. $\mathrm{m} / \mathrm{z} 315.1816$ [M $\left.-\mathrm{C}_{6} \mathrm{H}_{13} \mathrm{O}_{4}\right]^{-}$, calcd. $\mathrm{m} / \mathrm{z}$ 315.1813 for $\mathrm{C}_{16} \mathrm{H}_{27} \mathrm{O}_{6} ; \Delta=0.8 \mathrm{ppm}$; HPLC-ESI-(-)-MS : see Figure $\mathrm{S} 10 \mathrm{~b}$.

9-[(3'-[(3','6"'-Dideoxy-a-L-arabino-hexopyranosyl)-oxy]-butanoyl)oxy]-3,4-dihydroxytetradecanoic acid (asc-C4-5,6-(OH) $\left.)_{2}-\mathrm{C} 14\right)$ from $\mathrm{OsO}_{4}$ dihydroxylation of asc-C4-C14:1 (fasc\#16) from C. remanei PB4641: HPLC-ESI-(-)-HR-MS/MS: obsd. m/z 491.2848 [M - H] $]^{-}$, calcd. $\mathrm{m} / \mathrm{z} 491.2862$ for $\mathrm{C}_{24} \mathrm{H}_{43} \mathrm{O}_{10} ; \Delta=2.8$ ppm; obsd. $\mathrm{m} / \mathrm{z} 243.2113\left[\mathrm{M}-\mathrm{C}_{6} \mathrm{H}_{13} \mathrm{O}_{4}\right]^{-}$, calcd. $\mathrm{m} / \mathrm{z}$ 243.2126 for $\mathrm{C}_{18} \mathrm{H}_{31} \mathrm{O}_{6} ; \Delta=3.9$ ppm; HPLC-ESI-(-)-MS : see Figure S10c.

Analysis of sex-specific fatty acid ascaroside production. Young adults of $C$. remanei strain PB4641 from non-starved and non-crowded $6 \mathrm{~cm}$ NGM agar plates seeded with E. coli OP50 were sorted by sex using a "worm pick" made out of platinum iridium wire $(0.25 \mathrm{~mm}$ diameter $)$ and transferred to $6 \mathrm{~cm}$ peptone-free NGM agar plates. After checking once again for uniformity, 
exactly 150 male or female nematodes were transferred to $150 \mu \mathrm{S}$ S-medium supplemented with $1 \%$ E. coli OP50 (v/v) at $1 \mathrm{worm} / \mu \mathrm{l}$, and cultivated at $23{ }^{\circ} \mathrm{C}$ and $150 \mathrm{rpm}$. After $12 \mathrm{~h}$, worms and excess bacteria were removed by centrifugation $(5000 \mathrm{~g}$ for $10 \mathrm{~min}$ ) and the media supernatant was filtered over cotton, frozen at $-20^{\circ} \mathrm{C}$, lyophilized, and extracted with $2 \times 200 \mu$ methanol for $6 \mathrm{~h}$. Combined extracts were filtered over cotton, concentrated to dryness, and the residues dissolved in $200 \mu \mathrm{l}$ deionized water and absorbed on a preconditioned solid phase extraction cartridge (100 mg C18ec, Chromabond, Macherery Nagel). After washing with $2 \times 500 \mu l$ deionized water, nematode metabolites were eluted with $2 \times 500 \mu \mathrm{l}$ methanol. The solutions were concentrated to dryness under reduced pressure and the residues taken up in $60 \mu \mathrm{l}$ methanol for UHPLC-ESI-(-)-HR-MS/MS analysis (Figure S13).

Analysis of $\boldsymbol{C}$. remanei cyclopropyl fatty acid metabolism using $\boldsymbol{E}$. coli $\Delta c f a$. Mixed stage C. remanei strain PB4641 was cultivated on NGM agar plates seeded with either E. coli OP50 as control or the $E$. coli $\Delta c f a$ mutant for one week. Worms from five $10 \mathrm{~cm}$ plates each were collected in S-medium and transferred into $100 \mathrm{ml} \mathrm{S}$-medium supplemented with either $5 \mathrm{ml}$ concentrated E. coli OP $50,5 \mathrm{ml}$ concentrated $E$. coli $\Delta c f a$, or $5 \mathrm{ml}$ concentrated $E$. coli $\Delta c f a$ supplemented with synthetic potassium ( \pm )-cis-9,10-methylenehexadecanoate (cyC17, 25, $16.8 \mu \mathrm{mol})$. Cultures were grown at $20^{\circ} \mathrm{C}$ and $180 \mathrm{rpm}$. After 14 days, worms were removed by centrifugation (5000 g for $10 \mathrm{~min}$ ), washed once with $\mathrm{M} 9$ buffer, frozen at $-20^{\circ} \mathrm{C}$, lyophilized, and submitted for GC-MS analysis of their fatty acid methyl ester (FAME) composition (Figure S17). The media supernatant was filtered, frozen at $-20^{\circ} \mathrm{C}$, lyophilized, and extracted with $2 \times 50 \mathrm{ml}$ methanol. Extracts were filtered, concentrated to dryness at $40{ }^{\circ} \mathrm{C}$ under reduced pressure, and the residues submitted to NMR analysis. Aliquots of $10 \mu \mathrm{l}$ were submitted for comparative analysis by HPLC-ESI-(-)-HRMS (Figure S20). For each condition three biological replicates were analyzed and data shown in Figure $2 \mathrm{c}$ represent the calculated mean \pm SEM.

Isolation of cyclopropyl fatty acid catabolites. Exometabolome extracts from a $1.5 \mathrm{~L}$ Caenorhabditis remanei strain PB4641 culture or a $1.5 \mathrm{~L}$ Caenorhabditis briggsae strain AF16 culture (prepared as previously described) $)^{[4]}$ were fractionated by solid phase extraction on $5 \mathrm{~g}$ RP-C18 (Chromabond, Macherey Nagel) using a stepwise gradient of aqueous methanol (0 to $100 \%$ in $10 \%$ steps) as eluent. Fractions were concentrated under reduced pressure, dissolved in $500 \mu \mathrm{l} \mathrm{CD} \mathrm{CD}_{3} \mathrm{OD}$, and analyzed by ${ }^{1} \mathrm{H}$ NMR spectroscopy. Fractions rich in cis-cyclopropylfatty acid derivatives as indicated by characteristic signals around $\delta_{H}-0.20-0.10 \mathrm{ppm}$ were subsequently fractionated by either solid phase extraction on $500 \mathrm{mg} \mathrm{RP}-\mathrm{C} 18 \mathrm{ec}$ (Chromabond, Macherey Nagel) using a stepwise gradient of aqueous methanol as eluent ( 0 to $100 \%$ in $10 \%$ steps), 500 mg normal phase silica (Chromabond, Macherey Nagel) using a stepwise gradient of ethyl acetate in hexane (0 to $100 \%$ in $10 \%$ steps) as eluent, or semipreparative RP-C18-HPLC as described above.

cis-3,4-methylenedecanoic acid $(\operatorname{cyC} 11,26)$ isolated as a yellowish oil from the $C$. remanei PB4641 exometabolome ( 150 $\mu \mathrm{g})$; ${ }^{1} \mathrm{H}$ NMR $\left(400 \mathrm{MHz}, \mathrm{CD}_{3} \mathrm{OD}\right) \mathrm{\delta}:-0.01(1 \mathrm{H}$, ddd, $\mathrm{J}=9.6, \mathrm{~J}=\mathrm{J}$ $=4.5 \mathrm{~Hz}), 0.84(1 \mathrm{H}, \mathrm{ddd}, \mathrm{J}=9.6, \mathrm{~J}=8.1 \mathrm{~Hz}, 4.5 \mathrm{~Hz}), 0.93(1 \mathrm{H}, \mathrm{m}), 1.05(3 \mathrm{H}, \mathrm{t}, \mathrm{J}=7.1 \mathrm{~Hz}), 1.21$ $(1 \mathrm{H}, \mathrm{m}), 1.22(1 \mathrm{H}, \mathrm{m}), 1.36(1 \mathrm{H}, \mathrm{m}), 1.42-1.55(5 \mathrm{H}, \mathrm{m}), 1.46(1 \mathrm{H}, \mathrm{m}), 1.52(2 \mathrm{H}, \mathrm{m}), 2.36(1 \mathrm{H}$, dd, J = 16.3 Hz, J = 7.6 Hz), $2.44(1 \mathrm{H}, \mathrm{dd}, \mathrm{J}=16.3 \mathrm{~Hz}, \mathrm{~J}=7.7 \mathrm{~Hz}) ; \mathrm{HR}-\mathrm{MS}$ obsd. $\mathrm{m} / \mathrm{z} 183.1386$ $[\mathrm{M}-\mathrm{H}]^{-}$, calcd. $\mathrm{m} / \mathrm{z} 183.1391$ for $\mathrm{C}_{11} \mathrm{H}_{19} \mathrm{O}_{2}, \Delta=2.4 \mathrm{ppm}$. 
cis-3,4-methylenedecanedioic acid (cyC11DA, 27) isolated as a yellowish oil from the C. remanei PB4641 exometabolome $(\sim 125 \mu \mathrm{g}) ;{ }^{1} \mathrm{H}$ NMR $\left(400 \mathrm{MHz}, \mathrm{CD}_{3} \mathrm{OD}\right) \delta$ : $-0.13(1 \mathrm{H}$, ddd, J $=9.6, \mathrm{~J}=\mathrm{J}=4.5 \mathrm{~Hz}), 0.71(1 \mathrm{H}, \mathrm{ddd}, \mathrm{J}=9.6, \mathrm{~J}=8.1 \mathrm{~Hz}, 4.5 \mathrm{~Hz}), 0.81(1 \mathrm{H}, \mathrm{m}), 1.07(1 \mathrm{H}, \mathrm{m}), 1.22$ $(1 \mathrm{H}, \mathrm{m}), 1.38(1 \mathrm{H}, \mathrm{m}), 1.61(2 \mathrm{H}, \mathrm{m}), 2.26(1 \mathrm{H}, \mathrm{dd}, \mathrm{J}=16.4 \mathrm{~Hz}, \mathrm{~J}=7.6 \mathrm{~Hz}), 2.28(2 \mathrm{H}, \mathrm{t}, \mathrm{J}=7.5$ $\mathrm{Hz}), 2.32(1 \mathrm{H}, \mathrm{dd}, \mathrm{J}=16.4 \mathrm{~Hz}, \mathrm{~J}=7.4 \mathrm{~Hz})$; HR-MS obsd. $\mathrm{m} / \mathrm{z} 213.1130[\mathrm{M}-\mathrm{H}]^{-}$, calcd. $\mathrm{m} / \mathrm{z}$ 213.1132 for $\mathrm{C}_{11} \mathrm{H}_{17} \mathrm{O}_{4}, \Delta=1.2 \mathrm{ppm}$.

cis-3,4-methyleneoctanedioic acid (cyC9DA, 28) isolated as a yellowish oil from the $C$. remanei PB4641 exometabolome ( 465 $\mu \mathrm{g})$; ${ }^{1} \mathrm{H}$ NMR $\left(400 \mathrm{MHz}, \mathrm{CD}_{3} \mathrm{OD}\right) \delta:-0.10(1 \mathrm{H}$, ddd, $\mathrm{J}=9.4 \mathrm{~J}=\mathrm{J}$ $=4.5, \mathrm{~Hz}), 0.74(1 \mathrm{H}, \mathrm{ddd}, \mathrm{J}=9.4, \mathrm{~J}=8.4 \mathrm{~Hz}, 4.5 \mathrm{~Hz}), 0.83(1 \mathrm{H}, \mathrm{m}), 1.09(1 \mathrm{H}, \mathrm{m}), 1.24(1 \mathrm{H}, \mathrm{m})$, $1.46(1 \mathrm{H}, \mathrm{m}), 1.71(2 \mathrm{H}, \mathrm{m}), 2.26(1 \mathrm{H}, \mathrm{dd}, \mathrm{J}=16.3 \mathrm{~Hz}, \mathrm{~J}=7.4 \mathrm{~Hz}), 2.33(2 \mathrm{H}, \mathrm{t}, \mathrm{J}=7.6 \mathrm{~Hz}), 2.33$ $(1 \mathrm{H}, \mathrm{dd}, \mathrm{J}=16.3 \mathrm{~Hz}, \mathrm{~J}=7.3 \mathrm{~Hz}) ;{ }^{13} \mathrm{C}$ NMR (100 MHz, CD $\left.{ }_{3} \mathrm{OD}\right) \delta: 11.3,12.7,16.3,26.4,29.3$, 34.6, 34.8, 177.6; HR-MS obsd. $m / z 185.0819$ [M - H] $]^{-}$, calcd. $m / z 185.0819$ for $\mathrm{C}_{9} \mathrm{H}_{13} \mathrm{O}_{4}, \Delta=0.3$ ppm.

cis-3,4-methylenehexanedioic acid (cyC7DA, 29) isolated as a yellowish oil from the C. remanei PB4641 exometabolome $(\sim 825 \mu \mathrm{g}) ;{ }^{1} \mathrm{H}$ NMR $\left(\mathrm{CD}_{3} \mathrm{OD}\right) \delta: 0.04\left(1 \mathrm{H}, \mathrm{dt}{ }^{2} \mathrm{~J}=11.0 \mathrm{~Hz}\right.$, $5.7 \mathrm{~Hz}), 0.827(1 \mathrm{H}, \mathrm{dt} \mathrm{J}=5.0 \mathrm{~Hz}, \mathrm{~J}=8.5 \mathrm{~Hz}) 1.18(2 \mathrm{H}, \mathrm{m}), 2.27(2 \mathrm{H}, \mathrm{dd}, \mathrm{J}=16.5, \mathrm{~J}=7.1 \mathrm{~Hz})$, $2.36(2 \mathrm{H}, \mathrm{dd}, \mathrm{J}=16.3 \mathrm{~Hz}, \mathrm{~J}=7.2 \mathrm{~Hz})$; HR-MS obsd. $\mathrm{m} / \mathrm{z} 157.0513[\mathrm{M}-\mathrm{H}]^{-}$, calcd. $\mathrm{m} / \mathrm{z} 157.0506$ for $\mathrm{C}_{7} \mathrm{H}_{9} \mathrm{O}_{4}, \Delta=4.0 \mathrm{ppm}$.

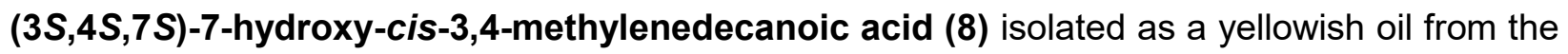
C. remanei PB4641 exometabolome $(\sim 20 \mu \mathrm{g}) ;{ }^{1} \mathrm{H}$ NMR $\left(400 \mathrm{MHz}, \mathrm{CD}_{3} \mathrm{OD}\right) \mathrm{\delta}:-0.16(1 \mathrm{H}, \mathrm{m}), 0.66$ $(1 \mathrm{H}, \mathrm{m}), 0.76(1 \mathrm{H}, \mathrm{m}), 0.93(3 \mathrm{H}, \mathrm{t}, \mathrm{J}=7.5 \mathrm{~Hz}), 1.12(1 \mathrm{H}, \mathrm{m}), 1.20-1.60(8 \mathrm{H}, \mathrm{m}), 2.15(1 \mathrm{H}, \mathrm{dd}, \mathrm{J}$ $=15.2 \mathrm{~Hz}, \mathrm{~J}=7.7 \mathrm{~Hz}), 2.26(1 \mathrm{H}, \mathrm{dd}, \mathrm{J}=15.1 \mathrm{~Hz}, \mathrm{~J}=6.8 \mathrm{~Hz}), 3.57(1 \mathrm{H}, \mathrm{m}) ; \mathrm{HR}-\mathrm{MS}$ obsd. $\mathrm{m} / \mathrm{z}$ 199.1339 [M - H] $]^{-}$, calcd. $m / z 199.1340$ for $\mathrm{C}_{11} \mathrm{H}_{19} \mathrm{O}_{3}, \Delta=0.2 \mathrm{ppm}$.

10-hydroxy-cis-3,4-methylenedecanoic acid isolated as a colorless oil from the $C$. remanei PB4641 exometabolome ( 20 $\mu \mathrm{g})$; ${ }^{1} \mathrm{H}$ NMR (400 MHz, $\left.\mathrm{CD}_{3} \mathrm{OD}\right) \delta:-0.17(1 \mathrm{H}, \mathrm{m}), 0.65(1 \mathrm{H}, \mathrm{m})$, $0.75(1 \mathrm{H}, \mathrm{m}), 0.92(1 \mathrm{H}, \mathrm{m}), 1.12(1 \mathrm{H}, \mathrm{m}), 1.14(3 \mathrm{H}, d, \mathrm{~J}=6.1 \mathrm{~Hz}), 1.20-1.60(8 \mathrm{H}, \mathrm{m}), 2.09(1 \mathrm{H}$, dd, J = 15.0 Hz, J = 7.5 Hz), $2.25(1 \mathrm{H}, \mathrm{dd}, \mathrm{J}=14.9 \mathrm{~Hz}, \mathrm{~J}=6.9 \mathrm{~Hz}), 3.71(1 \mathrm{H}, \mathrm{m})$; HR-MS obsd. $\mathrm{m} / \mathrm{z} 199.1343[\mathrm{M}-\mathrm{H}]^{-}$, calcd. $\mathrm{m} / \mathrm{z} 199.1340$ for $\mathrm{C}_{11} \mathrm{H}_{19} \mathrm{O}_{3}, \Delta=1.5 \mathrm{ppm}$.

Preparation of fatty acid methyl esters (FAMEs). $10 \mathrm{mg}$ of the lyophilized $E$. coli bacterial pellet or C. remanei worm pellet were treated with $1 \mathrm{ml}$ methanolic $2.5 \%$ sulfuric acid $(\mathrm{v} / \mathrm{v})$ and incubated at $70{ }^{\circ} \mathrm{C}$ for $1 \mathrm{~h}$. After returning to room temperature $1.5 \mathrm{ml}$ water and $200 \mu \mathrm{l}$ hexane were added, the mixture vortexed for $30 \mathrm{~s}$, and centrifuged at $200 \mathrm{~g}$ for $1 \mathrm{~min}$. The top organic layer containing the fatty acid methyl esters was collected for GC-EIMS analysis (Figures S17 and S19).

Gas Chromatography Electron Ionization Mass Spectrometry (GC-EIMS). Separation of volatiles and acquisition of their electron ionization mass spectra was performed using a Trace GC 2000 series (Thermo Scientific) equipped with a Zebron ZB-5 Guardian column (15 m, 0.25 $\mathrm{mm}$ ID, $0.25 \mu \mathrm{m}$ film thickness; with $10 \mathrm{~m}$ guardian end) coupled to a single quadrupole ThermoQuest Trace MS (Finnigan). Helium was used as the carrier gas at a flow rate of $1 \mathrm{ml} / \mathrm{min}$. A temperature program starting at $130{ }^{\circ} \mathrm{C}$ for $5 \mathrm{~min}$, followed by a linear gradient of $+10{ }^{\circ} \mathrm{C} / \mathrm{min}$ to $350^{\circ} \mathrm{C}$ was applied. A total volume of $1 \mu \mathrm{l}$ was injected using a 10:1 split ratio and an injector 
temperature of $250{ }^{\circ} \mathrm{C}$. Electron ionization (El, $70 \mathrm{eV}$ ) mass spectra were acquired from $\mathrm{m} / \mathrm{z}$ 35650 amu. Data were analyzed with the Xcalibur 3.1 software (Thermo Fisher Scientific).

Determination of Enantiomeric Excess by enantioselective Gas Chromatography. Enantioselective gaschromatography for the separation of chiral volatiles (Figures S1 and S2) and determination of their enantiomeric excess was performed using a Trace GC Ultra (Thermo Scientific) equipped with a heptakis-(2,3,6-tri-O-methyl)- $\beta$-cyclodextrin capillary column (25 $\mathrm{m} \mathrm{x}$ $0.25 \mathrm{~mm}$ ID, HYDRODEX $\beta-P M$, Macherey Nagel) or a heptakis-(2,3-di-O-methyl-6-O-tertbutyldimethylsilyl)- $\beta$-cyclodextrin capillary column (25 m x $0.25 \mathrm{~mm}$ ID, HYDRODEX $\beta-6 T B D M$, Macherey Nagel) coupled to a quadrupole ion trap ITQ 900 mass spectrometer (Thermo Scientific) for the acquisition of their electron ionization (El, $70 \mathrm{eV}$ ) mass spectra. Helium was used as the carrier gas at $1 \mathrm{~mL} / \mathrm{min}$. A total volume of $1 \mu \mathrm{l}$ was injected using a $10: 1$ split ratio and an injector temperature of $250{ }^{\circ} \mathrm{C}$. Separation conditions were optimized using the corresponding racemates. Data were analyzed with the Xcalibur 3.1 software (Thermo Fisher Scientific).

High Performance Liquid Chromatography - Electrospray lonization - High ResolutionTandem Mass Spectrometry (HPLC-ESI-HRMS/MS). HPLC-ESI-HRMS/MS analysis of crude nematode exometabolome extracts and exometabolome fractions was performed using a Dionex UltiMate 3000 HPLC instrument coupled to a Bruker Maxis high resolution qTOF mass spectrometer equipped with an electrospray ionization (ESI) unit operated in positive or negative mode. Chromatographic separations were achieved using an Agilent ZORBAX Eclipse XDB-C18 column ( $250 \times 3 \mathrm{~mm}, 5 \mu \mathrm{m}$ particle diameter) with a flow rate of $400 \mu \mathrm{l} / \mathrm{min}$ and gradient elution starting at $3 \%$ acetonitrile in $0.5 \%$ aqueous acetic acid for 5 minutes followed by a linear increase to $100 \%$ acetonitrile with $0.5 \%$ acetic acid within 35 minutes. Data were analyzed with the Compass DataAnalysis 4.3 software (Bruker Daltonics).

High Performance Liquid Chromatography - Electrospray Ionization - Tandem Mass Spectrometry Precursor lon Scanning. HPLC-MS/MS precursor ion scanning for $m / z 73.1$ was performed using an Agilent 1260 HPLC instrument (Agilent Technologies) coupled to an API5000 Triple Quadrupole LC/MS/MS mass spectrometer (AB Sciex, Darmstadt) equipped with an electrospray ionization (ESI) unit operated in negative mode. A CID energy of -34 units was applied. Chromatographic separations were achieved using an Agilent ZORBAX Eclipse XDBC18 column ( $50 \times 4.6 \mathrm{~mm}, 1.8 \mu \mathrm{m}$ particle diameter) (Agilent Technologies) with a flow rate of 1.1 $\mathrm{ml} / \mathrm{min}$ and gradient elution starting at $5 \%$ acetonitrile in $0.05 \%$ aqueous formic acid followed by a linear in-crease to $95 \%$ acetonitrile with $0.05 \%$ formic acid within 10 minutes. Data were analyzed with the Analyst 1.6 software (AB Sciex).

High Performance Liquid Chromatography - Electrospray lonization - Multipe Stage Mass Spectrometry (HPLC-ESI-(-)-MS ${ }^{n}$ ). HPLC-ESI-(-)-MS ${ }^{n}$ analyses were carried out using an Agilent HP1100 HPLC system coupled to a Finnigan LTQ (Thermo Electron Corp, Dreieich, Germany) equipped with an electrospray ionization (ESI) unit operated in negative mode. CID energies ranging from -30 to -60 units were applied. Chromatographic separations were achieved using an Agilent ZORBAX Eclipse XDB-C18 column ( $250 \times 3 \mathrm{~mm}, 5 \mu \mathrm{m}$ particle diameter) with a flow rate of $400 \mu \mathrm{l} / \mathrm{min}$ and gradient elution starting at $3 \%$ acetonitrile in $0.5 \%$ aqueous acetic acid 
for 5 minutes followed by a linear increase to $100 \%$ acetonitrile with $0.5 \%$ acetic acid within 35 minutes. Data were analyzed with the Xcalibur 3.1. software (Thermo Fisher Scientific). Data are shown in Table S5 and Figures S9-S10.

Ultra High Performance Liquid Chromatography-Electrospray lonization-High ResolutionTandem Mass Spectrometry (UHPLC-ESI-HRMS/MS). UHPLC-ESI-HRMS/MS analysis of crude nematode exometabolome extracts and exometabolome fractions was performed using an Acquity UHPLC instrument coupled to a Synapt G2 QTOF mass spectrometer (Waters, Milford, $M A, U S A)$ ) equipped with an electrospray ionization (ESI) probe operated in positive or negative mode. Chromatographic separations were achieved using a Waters Acquity UHPLC HSS T3 column (100 x $2.1 \mathrm{~mm}, 1.8 \mu \mathrm{m}$ particle diameter) with a flow rate of $500 \mu \mathrm{l} / \mathrm{min}$ and gradient elution starting at $100 \%$ aqueous formic acid $(0.05 \%)$ followed by a linear increase to $100 \%$ acetonitrile with $0.05 \%$ formic acid within 10 minutes. Data were analyzed with the MassLynx 4.1 software (Waters).

Nuclear Magnetic Resonance (NMR) spectroscopy. Standard NMR spectra (Figures S22S122) were recorded in $\mathrm{CD}_{3} \mathrm{OD}(99.8 \mathrm{D}$ or $99.95 \mathrm{D})$ or $\mathrm{CDCl}_{3}(99.8 \mathrm{D})$ at $400 \mathrm{MHz}$ for ${ }^{1} \mathrm{H}$ and 100 $\mathrm{MHz}$ for ${ }^{13} \mathrm{C}$ using a Bruker Avance III HD 400 instrument equipped with a $5 \mathrm{~mm}$ BBFO probe (MPICE) or Bruker Avance II 400 instrument equipped with a $5 \mathrm{~mm}$ BBFO probe (NPAC, UniNE), or at $700 \mathrm{MHz}$ for ${ }^{1} \mathrm{H}$ and $175 \mathrm{MHz}$ for ${ }^{13} \mathrm{C}$ using a Bruker Avance III HD 700 instrument equipped with a $1.7 \mathrm{~mm} \mathrm{TCl}$ microcryoprobe (MPICE). Residual solvent signals were used as internal standard with ${ }^{1} \mathrm{H}$ at $3.31 \mathrm{ppm}$ and ${ }^{13} \mathrm{C}$ at $49.05 \mathrm{ppm}$ for $\mathrm{CD}_{3} \mathrm{OD}$ or ${ }^{1} \mathrm{H}$ at $7.26 \mathrm{ppm}$ and ${ }^{13} \mathrm{C}$ at 77.16 ppm for $\mathrm{CDCl}_{3}$. Two-dimensional homonuclear double quantum filtered (dqf)-COSY spectra were recorded using phase cycling for coherence selection. For the isolated compounds a total of 32 scans were acquired using a time domain of 8k in F2 (acquisition time of $1.2 \mathrm{~s}$ ) and 512 increments in F1. For two-dimensional heteronuclear HSQC spectra 96 scans were acquired using a time domain of $1 \mathrm{k}$ in $\mathrm{F} 2$ and 256 increments in F1. Spectra were zero-filled to $8 \mathrm{k} \times 4 \mathrm{k}$ (COSY) or $4 \mathrm{k} \times 2 \mathrm{k}$ (HSQC) prior to Fourier transformation, phased manually, and baseline corrected using the Topspin 3.2 (Bruker Biospin) and MNova 9.0 (Mestrelab Research) software.

Optical rotation measurements. Optical rotation was measured using a Jasco P1020 Polarimeter at $598 \mathrm{~nm}$ with a Na lamp using a cell path length of $100 \mathrm{~mm}$. Each sample was measured ten times. The solutions of samples were prepared in HPLC grade chloroform.

Holding assay to evaluate nematode retention. Nematode preference for environments conditioned with known amounts of fatty acid ascarosides was measured using a modified retention assay. ${ }^{[4,5]}$ In a $6 \mathrm{~cm}$ Petri dish filled with $6 \mathrm{ml}$ peptone-free NGM agar, circular scoring regions of $9 \mathrm{~mm}$ diameter $\left(64 \mathrm{~mm}^{2}\right)$ were marked. Next, $1 \mu \mathrm{l}$ of $10 \%$ aqueous methanol (as solvent control) or fatty acid ascaroside solutions of asc-C4-cyC11 (fasc\#1, 3), asc-C4-C12:1 (fasc\#2, 4), or asc-C4-C10:1 (fasc\#3, 5) isolated from C. remanei PB4641, synthetic asc-C4 (ascr\#11, 19), or semisynthetic (3S,4S,7S)-7-hydroxy-cis-3,4-methylenedecanoic acid (8) obtained by hydrolysis of asc-C4-cyC11 (fasc\#1, 3), in biologically relevant concentration ranging from $10 \mathrm{pM}$ to $10 \mu \mathrm{M}$ dissolved in $10 \%$ aqueous methanol were placed in the center of the scoring areas onto the agar and left to dry for 5 minutes. Young adult nematodes from non-starved and non-crowded $6 \mathrm{~cm}$ NGM agar plates seeded with E. coli OP50 were sorted by sex and transferred to peptone-free 
NGM agar without food for approximately 30 min before being used for the assay to minimize the amount of concomitant bacteria. Individual worms (up to 3) were placed into the center of the conditioned scoring region and the time required for the nematodes to leave the scoring region was recorded. Nematodes were defined to have left the scoring area when no part of the nematode was still within the circular boundary. A total number of 20 worms per condition were analyzed and experiments were repeated on two separate days with comparable results. A oneway analysis of variance (ANOVA) with Dunnett's post-test was performed using the SPSS software (IBM Corp. Released 2013. IBM SPSS Statistics for Windows, Version 21.0. Armonk, NY: IBM Corp.) to evaluate if the presence of ascarosides significantly affected the mean times nematode spent in scoring regions.

\section{Total synthesis of fasc\#1 (asc-C4-cyC11)}

Synthesis of 5-O-tert-butyldimethylsilyloxy-pent-2-yn-1-ol.

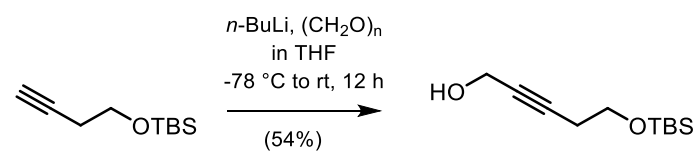

Under argon atmosphere a solution of $18.5 \mathrm{~g}$ of 4-O-tert-butyldimethylsilyloxy-1-butyne (100 $\mathrm{mmol}$ ) in $220 \mathrm{~mL}$ dry tetrahydrofuran at $-78{ }^{\circ} \mathrm{C}$ was treated with $50 \mathrm{~mL}$ of $2 \mathrm{M} n$-butyllithium solution $\left(100 \mathrm{mmol}\right.$ ) in pentane over the course of $2 \mathrm{~h}$. After stirring at $-78{ }^{\circ} \mathrm{C}$ for $45 \mathrm{~min}, 16 \mathrm{~g}$ paraformaldehyde (533 mmol) was added in one portion and the mixture was allowed to warm to room temperature. After stirring overnight, the reaction was quenched with $50 \mathrm{~mL}$ saturated aqueous $\mathrm{NH}_{4} \mathrm{Cl}$ solution, filtered, and extracted with diethyl ether. The organic layer was dried over $\mathrm{Na}_{2} \mathrm{SO}_{4}$, filtered, and concentrated under reduced pressure. Vacuum distillation (bp. $94{ }^{\circ} \mathrm{C}$ at $0.016 \mathrm{mbar}$ ) afforded $11.5 \mathrm{~g} \mathrm{5-O-tert-butyldimethylsilyloxy-pent-2-yn-1-ol}(53.6 \mathrm{mmol}, 54 \%$ yield) as a colorless oil. ${ }^{1} \mathrm{H}$ NMR $\left(400 \mathrm{MHz} \mathrm{CDCl}_{3}\right): \delta(\mathrm{ppm}) 0.05(6 \mathrm{H}, \mathrm{s}), 0.88(9 \mathrm{H}, \mathrm{s}), 2.17(1 \mathrm{H}$, s), $2.41(2 \mathrm{H}, \mathrm{tt}, \mathrm{J}=7.1 \mathrm{~Hz}, \mathrm{~J}=2.0 \mathrm{~Hz}), 3.70(2 \mathrm{H}, \mathrm{t}, \mathrm{J}=7.1 \mathrm{~Hz}), 4.21(2 \mathrm{H}, \mathrm{t}, \mathrm{J}=2.0 \mathrm{~Hz}) ;{ }^{13} \mathrm{C}\left\{{ }^{1} \mathrm{H}\right\}$ NMR (100 MHz, $\left.\mathrm{CDCl}_{3}\right)$ : $\delta$ (ppm) -5.2, 18.4, 23.2, 26.0, 51.3, 62.0, 79.7, 83.3.

\section{Synthesis of (Z)-5-O-tert-butyldimethylsilyloxy-pent-2-en-1-ol.}

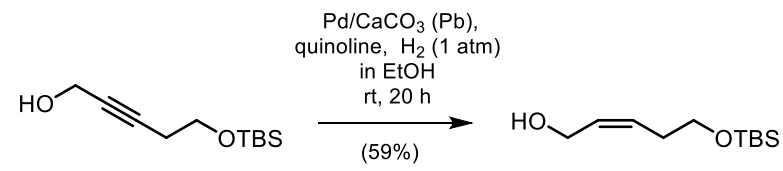

A solution of $11.5 \mathrm{~g}$ 5-O-tert-butyldimethylsilyloxy-pent-2-yn-1-ol $(53.6 \mathrm{mmol})$ in $40 \mathrm{ml}$ ethanol were treated with $1.7 \mathrm{~g}$ quinoline $(13.4 \mathrm{mmol}), 200 \mathrm{mg}$ Lindlar-catalyst $(2 \mathrm{~mol} \%)$ and $56 \mathrm{mg}$ potassium hydroxide $(1 \mathrm{mmol}, 2 \mathrm{~mol} \%)$. The resulting mixture was purged with hydrogen and stirred under 1 atm hydrogen atmosphere for $20 \mathrm{~h}$. After filtration over celite the solvent was removed under reduced pressure. Column chromatography on silica gel using $20 \%$ ethyl acetate in hexane (v/v) afforded $6.8 \mathrm{~g}(\mathrm{Z})$-5-O-tert-butyldimethylsilyloxy-pent-2-en-1-ol (31.4 mmol, $59 \%$ yield) as a colorless oil. $\left.{ }^{1} \mathrm{H} \mathrm{NMR} \mathrm{(400} \mathrm{MHz,} \mathrm{CDCl}_{3}\right): \delta(\mathrm{ppm}) 0.05(6 \mathrm{H}, \mathrm{s}), 0.88(9 \mathrm{H}$, $\mathrm{s}), 2.21(1 \mathrm{H}, \mathrm{s}), 2.33(2 \mathrm{H}, \mathrm{tdd}, \mathrm{J}=\mathrm{J}=6.6 \mathrm{~Hz}, \mathrm{~J}=1.3 \mathrm{~Hz}), 3.63(1 \mathrm{H}, \mathrm{t}, \mathrm{J}=6.2 \mathrm{~Hz}), 4.13(2 \mathrm{H}$, dd, $\mathrm{J}=6.7 \mathrm{~Hz}, \mathrm{~J}=1.2 \mathrm{~Hz}), 5.56\left(1 \mathrm{H}, \mathrm{dtt}, \mathrm{J}_{\mathrm{z}}=10.9 \mathrm{~Hz}, \mathrm{~J}=7.9 \mathrm{~Hz}, \mathrm{~J}=1.3 \mathrm{~Hz}\right), 5.80\left(1 \mathrm{H}, \mathrm{dtt}, \mathrm{J}_{\mathrm{z}}=10.9\right.$ 
$\mathrm{Hz}, \mathrm{J}=6.8 \mathrm{~Hz}, \mathrm{~J}=1.3 \mathrm{~Hz}) ;{ }^{13} \mathrm{C}\left\{{ }^{1} \mathrm{H}\right\} \operatorname{NMR}\left(100 \mathrm{MHz}, \mathrm{CDCl}_{3}\right): \delta(\mathrm{ppm})-5.3,18.6,26.1,31.0,58.2$, $62.4,129.8,131.0$.

Synthesis of (-)-(2S,3S)-5-O-tert-butyldimethylsilyloxy-cis-2,3-methylenepentan-1-ol (12).

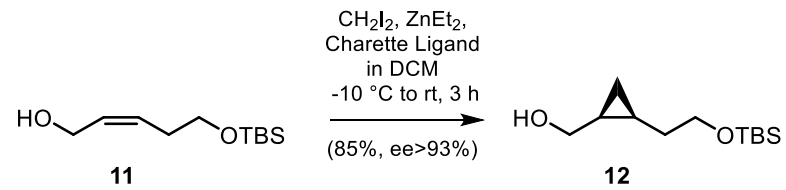

(This reactions is not to be scaled up). ${ }^{[6]}$ Under argon atmosphere a solution of $10 \mathrm{~mL} 1 \mathrm{M}$ diethyl zinc $(10 \mathrm{mmol})$ in hexane in $50 \mathrm{~mL}$ dry dichloromethane at $-10{ }^{\circ} \mathrm{C}$ was treated with $3 \mathrm{~mL}$

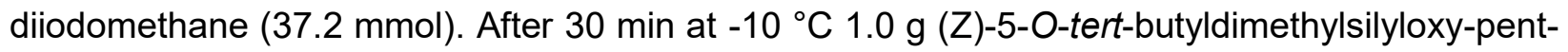
2-en-1-ol (11, $4.6 \mathrm{mmol})$ was added and after another $10 \mathrm{~min} 1.6 \mathrm{~g}$ of the $(R, R)-(+)-N, N, N^{\prime}, N^{\prime}$-tetramethyltartaric acid diamide $(5 \mathrm{mmol})$ in $1 \mathrm{~mL}$ dry dichloromethane was added. The reaction was allowed to return to room temperature and after $3 \mathrm{~h}$ the reaction was quenched by addition of $1 \mathrm{~mL}$ saturated aqueous $\mathrm{NH}_{4} \mathrm{Cl}$ solution, dried over $\mathrm{Na}_{2} \mathrm{SO}_{4}$, filtered, and concentrated under reduced pressure. Column chromatography on silica gel using $20 \%$ ethyl acetate in hexane (v/v) afforded $0.9 \mathrm{~g}(-)-(2 S, 3 S)-5-O-t e r t-b u t y l d i m e t h y l s i l y l o x y-c i s-2,3-$ methylenepentan-1-ol (12, $3.9 \mathrm{mmol}, 85 \%$ yield) as a colorless oil. Enantioselective gas chromatography using heptakis(2,3-O-dimethyl-6-O-tert-butyldimethylsilyl)- $\beta$-cyclodextrin as chiral phase showed an enantiomeric excess of ee $>93 \%$ (Figure S1). ${ }^{1} \mathrm{H} \mathrm{NMR}\left(400 \mathrm{MHz}, \mathrm{CDCl}_{3}\right)$ : $\delta(\mathrm{ppm})-0.10(1 \mathrm{H}, \mathrm{m}), 0.087(3 \mathrm{H}, \mathrm{s}), 0.093(3 \mathrm{H}, \mathrm{s}), 0.64(1 \mathrm{H}, \mathrm{ddd}, \mathrm{J}=\mathrm{J}=8.4 \mathrm{~Hz}, \mathrm{~J}=4.8 \mathrm{~Hz})$, $0.75(1 \mathrm{H}, \mathrm{m}), 0.91(9 \mathrm{H}, \mathrm{s}), 1.22(1 \mathrm{H}, \mathrm{m}), 1.45(1 \mathrm{H}, \mathrm{m}, \mathrm{J}=7.4 \mathrm{~Hz}, \mathrm{~J}=10.0 \mathrm{~Hz}), 1.80(1 \mathrm{H}, \mathrm{ddd}, \mathrm{J}=$ 14.7, J = 7.4 Hz, J = 3.8), 3.21 (1H, ddd, J = 12.0, J = $10.6 \mathrm{~Hz}, \mathrm{~J}=2.2 \mathrm{~Hz}), 3.68(1 \mathrm{H}, \mathrm{ddd}, \mathrm{J}=\mathrm{J}$ $=10.2, \mathrm{~J}=3.6), 3.81(1 \mathrm{H}, \mathrm{m}), 3.85(1 \mathrm{H}, \mathrm{dd}, \mathrm{J}=9.7 \mathrm{~Hz}, \mathrm{~J}=5.5 \mathrm{~Hz}) ;{ }^{13} \mathrm{C}\left\{{ }^{1} \mathrm{H}\right\} \mathrm{NMR}(100 \mathrm{MHz}$, $\left.\mathrm{CDCl}_{3}\right): \delta(\mathrm{ppm})-5.4,-5.3,7.6,13.7,18.6,18.8,26.2,30.8,62.6,64.6$; $[\alpha]^{24.3}=-30.9^{\circ}(\mathrm{c}=1$ $\left.\mathrm{mg} / \mathrm{mL}, \mathrm{CHCl}_{3}\right)$.

\section{Synthesis of (2S,3S)-5-O-tert-butyldimethylsilyloxy-cis-2,3-methylenepentanal (13).}

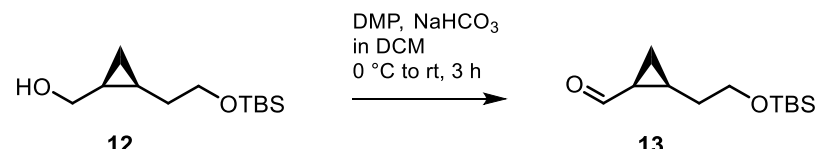

Under argon atmosphere a solution of $276 \mathrm{mg}$ Dess-Martin-Periodinane $(65 \mu \mathrm{mol})$ and $20 \mathrm{mg}$ of sodium hydrogencarbonate $(220 \mu \mathrm{mol})$ in $1 \mathrm{~mL}$ dry dichloromethane at $0{ }^{\circ} \mathrm{C}$ was treated with 10 $\mathrm{mg}$ of (2S,3S)-5-O-tert-butyldimethylsilyloxy-cis-2,3-methylenepentan-1-ol (12, $43 \mu \mathrm{mol})$ in $1 \mathrm{~mL}$ dry dichloromethane and allowed to return to room temperature. After $3 \mathrm{~h}$ the reaction was quenched with $200 \mu \mathrm{L}$ saturated aqueous sodium thiosulfate solution, dried over $\mathrm{MgSO}_{4}$, filtered, and concentrated under reduced pressure. The resulting (2S,3S)-5-O-tert-butyldimethylsilyloxycis-2,3-methylenepentanal (13) was immediately used for the next step without further purification. ${ }^{1} \mathrm{H}$ NMR $\left(400 \mathrm{MHz}, \mathrm{CDCl}_{3}\right): \delta(\mathrm{ppm}) 0.05(6 \mathrm{H}, \mathrm{s}), 0.89(9 \mathrm{H}, \mathrm{s}), 1.21(2 \mathrm{H}, \mathrm{m}), 1.60$ $(1 \mathrm{H}, \mathrm{m}), 1.71(1 \mathrm{H}, \mathrm{m}), 1.81(1 \mathrm{H}, \mathrm{m}), 1.91(1 \mathrm{H}, \mathrm{m}), 3.65(2 \mathrm{H}, \mathrm{m}), 9.41(1 \mathrm{H}, d, \mathrm{~J}=5.2 \mathrm{~Hz}) ;{ }^{13} \mathrm{C}\left\{{ }^{1} \mathrm{H}\right\}$ $\operatorname{NMR}\left(100 \mathrm{MHz}, \mathrm{CDCl}_{3}\right): \delta(\mathrm{ppm})-5.2,14.2,18.5,21.9,26.1,27.4,31.2,63.1,201.6$. 
Synthesis of (-)-(3R,4S)-6-O-tert-butyldimethylsilyloxy-cis-3,4-methylene-1-hexene (14).

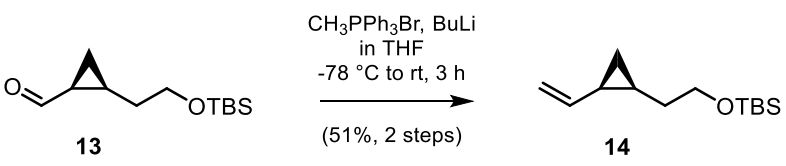

Under argon atmosphere a suspension of $350 \mathrm{mg}(1.0 \mathrm{mmol})$ methyltriphenylphosphonium bromide in $25 \mathrm{~mL}$ dry tetrahydrofuran at $-78{ }^{\circ} \mathrm{C}$ was treated with $350 \mu \mathrm{L}(0.87 \mathrm{mmol})$ of a $2.5 \mathrm{M}$ $n$-butyl lithium solution in hexane. After stirring at $-78{ }^{\circ} \mathrm{C}$ for $30 \mathrm{~min}$ the clear yellow solution was treated with $90 \mathrm{mg}(2 S, 3 S)-5-O-t e r t-b u t y l d i m e t h y l s i l y l o x y-2,3-c i s-m e t h y l e n e p e n t a n a l ~(13,0.39$ $\mathrm{mmol}$ ) in $500 \mu \mathrm{L}$ dry tetrahydrofuran. The reaction was stirred at $-78{ }^{\circ} \mathrm{C}$ for $1 \mathrm{~h}$ and then warmed to room temperature for an additional $2 \mathrm{~h}$. The reaction was quenched by addition of $1 \mathrm{~mL}$ saturated aqueous $\mathrm{NH}_{4} \mathrm{Cl}$ solution. The organic phase was separated, dried over $\mathrm{Na}_{2} \mathrm{SO}_{4}$, and concentrated under reduced pressure. Column chromatography on silica gel using $1 \%$ diethyl ether in pentane (v/v) afforded $45.3 \mathrm{mg}(-)-(3 S, 4 R)-6$-O-tert-butyldimethylsilyloxy-cis-3,4methylene-1-hexene $\left(14,0.20 \mathrm{mmol}, 51 \%\right.$ over two steps) as a colorless oil. ${ }^{1} \mathrm{H}$ NMR $(400 \mathrm{MHz}$, $\left.\mathrm{CDCl}_{3}\right): \delta(\mathrm{ppm}) 0.05(6 \mathrm{H}, \mathrm{s}), 0.26(1 \mathrm{H}, \mathrm{m}), 0.87(1 \mathrm{H}, \mathrm{m}), 0.90(9 \mathrm{H}, \mathrm{s}), 1.02(1 \mathrm{H}, \mathrm{m}), 1.29(1 \mathrm{H}, \mathrm{m})$, $1.52(2 \mathrm{H}, \mathrm{m}), 3.66(2 \mathrm{H}, \mathrm{m}), 4.97(1 \mathrm{H}, \mathrm{dd}, \mathrm{J}=10.3 \mathrm{~Hz}, \mathrm{~J}=1.6 \mathrm{~Hz}), 5.11(1 \mathrm{H}, \mathrm{dd}, \mathrm{J}=17.1 \mathrm{~Hz}, \mathrm{~J}=$ $1.9 \mathrm{~Hz}), 5.56(1 \mathrm{H}, \mathrm{dt}, \mathrm{J}=17.1 \mathrm{~Hz}, \mathrm{~J}=9.4 \mathrm{~Hz}) ;{ }^{13} \mathrm{C}\left\{{ }^{1} \mathrm{H}\right\} \mathrm{NMR}\left(100 \mathrm{MHz}, \mathrm{CDCl}_{3}\right): \delta(p p m)-5.1$, $12.4,15.5,18.5,19.5,26.2,32.6,63.4,114.3,138.4 ;[\alpha]^{20}=-21.0^{\circ}$ (c = 2.6 mg/mL, $\mathrm{CHCl}_{3}$ ).

\section{Synthesis of $( \pm)-1-h e x e n-3-y l$ acetate $(( \pm)-15)$.}

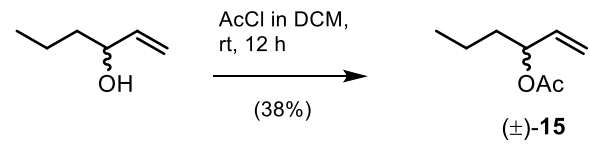

Under argon atmosphere a solution of $100 \mathrm{mg}$ racemic $( \pm)-1$-hexen-3-ol $(1 \mathrm{mmol})$ in $2 \mathrm{~mL}$ dry dichloromethane at $0{ }^{\circ} \mathrm{C}$ was treated with $85 \mu \mathrm{L}(1.2 \mathrm{mmol})$ acetyl chloride. After stirring at room temperature for $12 \mathrm{~h}$ the solution was concentrated under reduced pressure. Column chromatography on silica gel using $7 \%$ ethyl acetate in hexane yielded $54 \mathrm{mg}( \pm)$-1-hexen-3-yl acetate $(( \pm)-15,0.38 \mathrm{mmol}, 38 \%$ yield $)$ as a colorless oil.

\section{Synthesis of (-)-(3S)-1-hexen-3-yl acetate ((-)-15).}

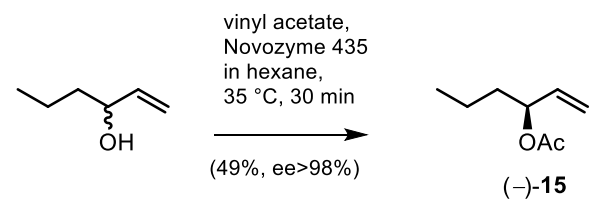

A solution of $100 \mathrm{mg}$ racemic $( \pm)$-1-hexen-3-ol $(1 \mathrm{mmol})$ in $2 \mathrm{~mL}$ hexane was treated with $100 \mu \mathrm{L}$ vinyl acetate and $20 \mathrm{mg}$ immobilized Candida antarctica lipase B (Novozym 435). The mixture was stirred for $30 \mathrm{~min}$ at $35{ }^{\circ} \mathrm{C}$. After filtration, the solution was concentrated under reduced pressure and purified by column chromatography on silica gel using $5 \%$ ethyl acetate in hexane (v/v) to afford $35 \mathrm{mg}(-)-(3 S)-1-$ hexen-3-yl acetate ((-)-15, $25 \mathrm{mmol}, 49 \%$ yield) with an enantiomeric excess of ee $>98 \%$ as determined by enantioselective GC analysis using a chiral 
heptakis-(2,3,6-tri-O-methyl)- $\beta$-cyclodextrin column (25 m x $0.25 \mathrm{~mm}$ ID, HYDRODEX $\beta$-PM, Macherey Nagel, Figure S2). ${ }^{1} \mathrm{H}$ NMR $\left(400 \mathrm{MHz}, \mathrm{CDCl}_{3}\right): \delta(\mathrm{ppm}) 0.90(3 \mathrm{H}, \mathrm{m}), 1.32(2 \mathrm{H}, \mathrm{m})$, $1.56(2 \mathrm{H}, \mathrm{m}), 2.03(3 \mathrm{H}, \mathrm{s}), 5.13(1 \mathrm{H}, \mathrm{m}), 5.20(1 \mathrm{H}, \mathrm{m}), 5.21(1 \mathrm{H}, \mathrm{m}), 5.75(1 \mathrm{H}, \mathrm{m}) ;{ }^{13} \mathrm{C}\left\{{ }^{1} \mathrm{H}\right\} \mathrm{NMR}$ $\left(100 \mathrm{MHz}_{\mathrm{CDCl}}\right): \delta$ (ppm) 13.9, 18.4, 21.3, 36.4, 74.7, 116.5, 136.8, 170.4.

Synthesis of $(E)-(3 S, 4 S, 7 S)-1-0$-tert-butyldimethylsilyloxy-cis-3,4-methylenedec-5-en-7-yl acetate (16).

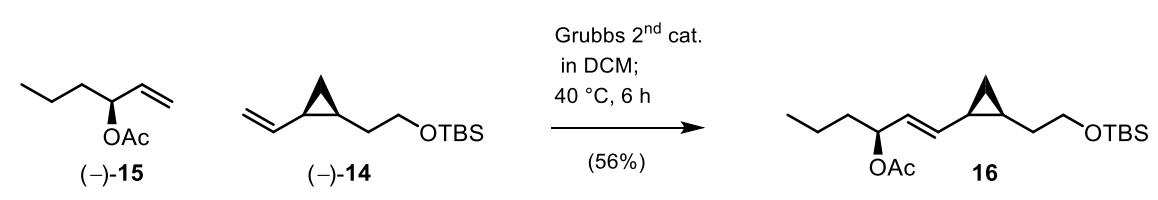

Under argon atmosphere a solution of $30 \mathrm{mg}(-)-(3 S, 4 R)-6-O-t e r t-b u t y l d i m e t h y l s i l y l o x y-c i s-$ 3,4-methylene-1-hexene (14, $132 \mu \mathrm{mol})$ and $94 \mathrm{mg}(-)-(3 S)-1-$ hexen-3-yl acetate (15) (662 $\mu \mathrm{mol})$ in $1 \mathrm{~mL}$ dichloromethane at reflux was treated with $11 \mathrm{mg}$ Grubbs $2^{\text {nd }}$ generation catalyst $(13 \mu \mathrm{mol})$. After stirring at reflux for $6 \mathrm{~h}$ the reaction mixture was concentrated under reduced pressure. Column chromatography on silica gel using $10 \%$ ethyl acetate in hexane (v/v) afforded $25 \mathrm{mg}(E)$-(3S,4S,7S)-1-O-tert-butyldimethylsilyloxy-cis-3,4-methylenedec-5-en-7-yl acetate $\left(16,73 \mathrm{mmol}, 56 \%\right.$ yield) as a colorless oil. ${ }^{1} \mathrm{H}$ NMR $\left(400 \mathrm{MHz}, \mathrm{CDCl}_{3}\right): \delta(\mathrm{ppm}) 0.04(6 \mathrm{H}$, s), $0.26(1 \mathrm{H}, \mathrm{m}), 0.89(9 \mathrm{H}, \mathrm{s}), 0.89(1 \mathrm{H}, \mathrm{m}) 0.91(3 \mathrm{H}, \mathrm{t}, \mathrm{J}=6.9 \mathrm{~Hz}), 1.03(1 \mathrm{H}, \mathrm{m}), 1.30(2 \mathrm{H}, \mathrm{m})$, $1.43(1 \mathrm{H}, \mathrm{ddd}, \mathrm{J}=16.7 \mathrm{~Hz}, \mathrm{~J}=8.4 \mathrm{~Hz}, \mathrm{~J}=5.4 \mathrm{~Hz}), 1.52(3 \mathrm{H}, \mathrm{m}), 1.61(3 \mathrm{H}, \mathrm{m}), 2.02(3 \mathrm{H}, \mathrm{s}), 3.63$ $(2 \mathrm{H}, \mathrm{td}, \mathrm{J}=6.8 \mathrm{~Hz}, \mathrm{~J}=2.4 \mathrm{~Hz}), 5.19(1 \mathrm{H}, \mathrm{q}, \mathrm{J}=6.9 \mathrm{~Hz}), 5.43(1 \mathrm{H}, \mathrm{dd}, \mathrm{J}=15.2 \mathrm{~Hz}, \mathrm{~J}=8.4 \mathrm{~Hz})$ $5.50(1 \mathrm{H}, \mathrm{dd}, \mathrm{J}=15.2 \mathrm{~Hz}, \mathrm{~J}=7.3 \mathrm{~Hz}) ;{ }^{13} \mathrm{C} \mathrm{NMR}\left(100 \mathrm{MHz}, \mathrm{CDCl}_{3}\right)$ : $\delta$ (ppm) -5.1, 14.0, 15.6, 18.2, $18.7,21.6,26.1(3), 32.7,36.9,63.3,75.1,126.6,128.6,134.5,170.6$.

Synthesis of $\quad(3 S, 4 S, 7 S)-1-0$-tert-butyldimethylsilyloxy-cis-3,4-methylenedecan-7-yl acetate (17).

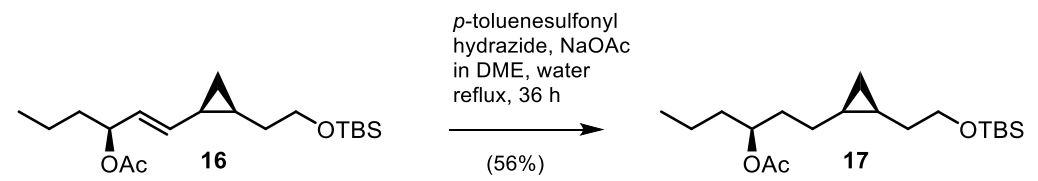

A solution of $5.0 \mathrm{mg}(E)$-(3S,4S,7S)-1-O-tert-butyldimethylsilyloxy-cis-3,4-methylenedec-5-en-7$\mathrm{yl}$ acetate $(16,14.7 \mu \mathrm{mol})$ and $25 \mathrm{mg} p$-toluenesulfonylhydrazide $(150 \mu \mathrm{mol})$ in $500 \mu \mathrm{L} 1$,2dimethoxyethane at reflux was treated with $20 \mathrm{mg}$ sodium acetate $(240 \mu \mathrm{mol})$ in $200 \mu \mathrm{L}$ water. After $36 \mathrm{~h}$ at reflux the reaction was concentrated under reduced pressure. Column chromatography on silica gel using $10 \%$ ethyl acetate in hexane (v/v) afforded $2.7 \mathrm{mg}(3 S, 4 S, 7 S)$ 1-O-tert-butyldimethylsilyloxy-cis-3,4-methylenedecan-7-yl acetate (17, 7.9 $\mu \mathrm{mol}, 56 \%$ yield) as a colorless oil. ${ }^{1} \mathrm{H}$ NMR $\left(400 \mathrm{MHz}, \mathrm{CDCl}_{3}\right): \delta(\mathrm{ppm})-0.27(1 \mathrm{H}, \mathrm{td}, \mathrm{J}=5.4 \mathrm{~Hz}, \mathrm{~J}=4.4 \mathrm{~Hz}), 0.06(6 \mathrm{H}$, s), $0.60(1 \mathrm{H}, \mathrm{m}), 0.71(2 \mathrm{H}, \mathrm{m}), 0.90(9 \mathrm{H}, \mathrm{s}), 0.90(3 \mathrm{H}, \mathrm{t}, \mathrm{J}=7.3 \mathrm{~Hz}), 1.18(1 \mathrm{H}, \mathrm{m}), 1.34(4 \mathrm{H}, \mathrm{m})$, $1.50(2 \mathrm{H}, \mathrm{m}), 1.64(2 \mathrm{H}, \mathrm{m}), 2.03(3 \mathrm{H}, \mathrm{s}), 3.67(2 \mathrm{H}, \mathrm{td}, \mathrm{J}=7.0 \mathrm{~Hz}, \mathrm{~J}=1.2 \mathrm{~Hz}), 4.91(1 \mathrm{H}, \mathrm{m}) ;{ }^{13} \mathrm{C}$ $\operatorname{NMR}\left(100 \mathrm{MHz}, \mathrm{CDCl}_{3}\right): \delta$ (ppm) $-5.1,10.7,12.6,14.1,15.3,18.6,18.7,21.4,24.7,26.2,32.2$, 34.6, 36.4, 63.9, 74.2, 171.0 . 
Synthesis of (3S,4S,7S)-1-O-tert-butyldimethylsilyloxy-cis-3,4-methylenedecan-7-ol (18).
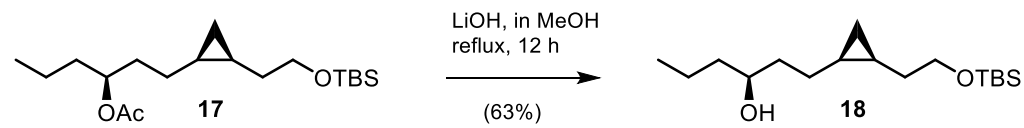

A solution of $10 \mathrm{mg}$ (3S,4S,7S)-1-O-tert-butyldimethylsilyloxy-cis-3,4-methylenedecan-7-yl acetate $(17,29.2 \mu \mathrm{mol})$ in $2 \mathrm{ml}$ methanol was treated with $5 \mu \mathrm{L}$ of a saturated aqueous lithium hydroxide solution and stirred at reflux overnight. After adjusting the $\mathrm{pH}$ at 7 with acetic acid the solution was concentrated under reduced pressure. Column chromatography on silica gel using $15 \%$ ethyl acetate in hexane ( $/ \mathrm{v})$ afforded $5.5 \mathrm{mg}(3 S, 4 S, 7 S)-1-O-t e r t-b u t y l d i m e t h y l s i l y l o x y-c i s-$ 3,4-methylenedecan-7-ol $\left(\mathbf{1 8}, 18.3 \mu \mathrm{mol}, 63 \%\right.$ yield) as a colorless oil. ${ }^{1} \mathrm{H} \mathrm{NMR}\left(400 \mathrm{MHz}, \mathrm{CDCl}_{3}\right)$ : $\delta(\mathrm{ppm})-0.25(1 \mathrm{H}, \mathrm{m}), 0.06(6 \mathrm{H}, \mathrm{s}), 0.61(1 \mathrm{H}, \mathrm{m}), 0.73(2 \mathrm{H}, \mathrm{m}), 0.90(9 \mathrm{H}, \mathrm{s}), 0.93(3 \mathrm{H}, \mathrm{t}, \mathrm{J}=7.0$ $\mathrm{Hz}), 1.32(2 \mathrm{H}, \mathrm{m}), 1.43(6 \mathrm{H}, \mathrm{m}), 1.55(1 \mathrm{H}, \mathrm{m}), 1.68(1 \mathrm{H}, \mathrm{m}), 3.65(1 \mathrm{H}, \mathrm{s}), 3.69(2 \mathrm{H}, \mathrm{t}, \mathrm{J}=6.7 \mathrm{~Hz})$; ${ }^{13} \mathrm{C}$ NMR $\left(100 \mathrm{MHz}, \mathrm{CDCl}_{3}\right): \delta$ (ppm) $-5.1(2), 10.7,12.6,14.1,15.3,18.6,18.7,21.4,24.7,26.1$ (3), 32.2, 34.6, 36.4, 63.9, 74.2, 171.0; ESI-HRMS: obsd. $\mathrm{m} / \mathrm{z}=323.2381[\mathrm{M}+\mathrm{Na}]^{+}$, calcd. $\mathrm{m} / \mathrm{z}=$ 323.2377 for $\mathrm{C}_{17} \mathrm{H}_{36} \mathrm{NaO}_{2} \mathrm{Si}, \Delta=1.2 \mathrm{ppm}$.

Synthesis of (3R)-methyl 3-[(3,6-dideoxy- $\alpha$-L-arabino-hexopyranosyl)oxy]-butanoate.
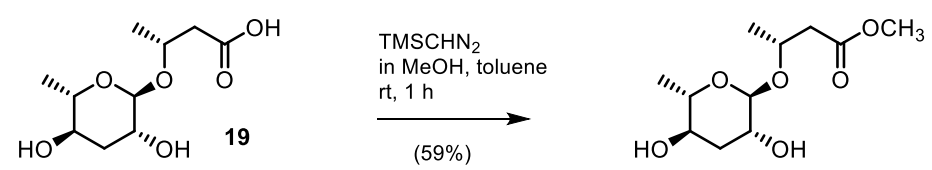

A solution of $62.2 \mathrm{mg}(3 R)-3-[(3,6-$ dideoxy- $\alpha$-L-arabino-hexopyranosyl)oxy]-butanoic acid (ascC4, ascr\#11, 19, $265 \mu \mathrm{mol}$ ) (obtained in 8 steps from L-rhamnose and ethyl (3R)hydroxybuytyrate as previously described $)^{[7]}$ in $1 \mathrm{~mL}$ of a $1: 1$ mixture $(\mathrm{v} / \mathrm{v})$ of methanol and toluene was treated with $250 \mu \mathrm{L}$ of a $2 \mathrm{M}$ (trimethylsilyl)diazomethane solution (500 $\mu \mathrm{mol})$ in diethyl ether. After stirring for $1 \mathrm{~h}$ excess reagent was quenched by addition of $30 \mu \mathrm{L}$ acetic acid (500 $\mu \mathrm{mol})$ and the solution concentrated under reduced pressure. Column chromatography on silica gel using $10 \%$ methanol in dichloromethane afforded $37.8 \mathrm{mg}(3 R)$-methyl 3-[(3,6-dideoxy- $\alpha$-Larabino-hexo-pyranosyl)oxy]-butanoate $\left(152 \mu \mathrm{mol}, 59 \%\right.$ yield) as a colorless oil. ${ }^{1} \mathrm{H}$ NMR (400 $\left.\mathrm{MHz}, \mathrm{CD}_{3} \mathrm{OD}\right): \delta(\mathrm{ppm}) 1.20(6 \mathrm{H}, \mathrm{m}), 1.70(1 \mathrm{H}, \mathrm{m}), 1.92(1 \mathrm{H}, \mathrm{td}, \mathrm{J}=12.8 \mathrm{~Hz}, \mathrm{~J}=3.7 \mathrm{~Hz}), 2.52$ $(2 \mathrm{H}, \mathrm{m}), 3.52(2 \mathrm{H}, \mathrm{m}), 3.68(3 \mathrm{H}, \mathrm{s}), 3.70(1 \mathrm{H}, \mathrm{br} s), 4.25(1 \mathrm{H}, \mathrm{m}), 4.65(1 \mathrm{H}, \mathrm{s}) ;{ }^{13} \mathrm{C}\left\{{ }^{1} \mathrm{H}\right\} \mathrm{NMR}(100$ $\left.\mathrm{MHz}, \mathrm{CD}_{3} \mathrm{OD}\right): \delta$ (ppm) 18.0, 19.2, 35.8, 43.2, 52.1, 68.2, 69.76, 69.79, 71.2, 97.5, 173.5.

\section{Synthesis of (3R)-methyl 3-[(2,4-di-O-tert-butyldimethylsilyloxy-3,6-dideoxy-a-L-arabino-} hexopyranosyl)oxy]-butanoate.
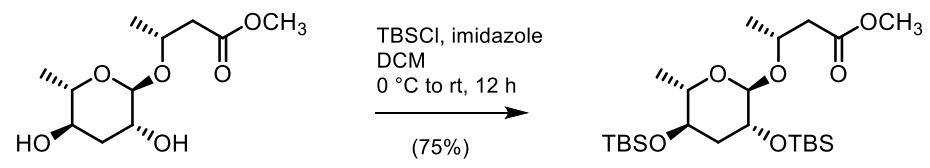

A solution of $20 \mathrm{mg}(3 R)$-methyl 3-[(3,6-dideoxy-a-L-arabino-hexopyranosyl)oxy]-butanoate (81 $\mu \mathrm{mol})$ in $2 \mathrm{ml}$ dry dichloromethane at $0{ }^{\circ} \mathrm{C}$ was treated with $72 \mathrm{mg}$ imidazole $(0.4 \mathrm{mmol})$ and 61 $\mathrm{mg}$ tert-butyldimethylsilyl chloride $(0.4 \mathrm{mmol})$. After stirring at room temperature for $12 \mathrm{~h}$ the 
reaction was washed with $3 \times 2 \mathrm{ml}$ water, dried over $\mathrm{Na}_{2} \mathrm{SO}_{4}$ and concentrated under reduced pressure. Column chromatography on silica gel using $5 \%$ methanol in dichloromethane afforded $28.6 \quad \mathrm{mg} \quad(3 R)$-methyl 3-[(2,4-di-O-tert-butyldimethylsilyloxy-3,6-dideoxy- $\alpha$-L-arabinohexopyranosyl) oxy]-butanoate $\left(60 \mu \mathrm{mol}, 75 \%\right.$ yield) as a colorless oil. ${ }^{1} \mathrm{H}$ NMR $(400 \mathrm{MHz}$, $\left.\mathrm{CD}_{3} \mathrm{OD}\right): \delta(\mathrm{ppm}) 0.08(12 \mathrm{H}, \mathrm{m}), 0.90(9 \mathrm{H}, \mathrm{s}), 0.92(9 \mathrm{H}, \mathrm{s}), 1.16(3 \mathrm{H}, \mathrm{d}, \mathrm{J}=6.2 \mathrm{~Hz}), 1.18(3 \mathrm{H}, \mathrm{d}$, $\mathrm{J}=6.2 \mathrm{~Hz}), 1.70(1 \mathrm{H}, \mathrm{ddd}, \mathrm{J}=13.0 \mathrm{~Hz}, \mathrm{~J}=10.6 \mathrm{~Hz}, \mathrm{~J}=2.6 \mathrm{~Hz}), 1.81(1 \mathrm{H}, \mathrm{ddd}, \mathrm{J}=12.8 \mathrm{~Hz}, \mathrm{~J}=$ $3.8 \mathrm{~Hz}, \mathrm{~J}=3.8 \mathrm{~Hz}), 2.51(2 \mathrm{H}, \mathrm{d}, \mathrm{J}=6.7 \mathrm{~Hz}), 3.52(1 \mathrm{H}, \mathrm{dq}, \mathrm{J}=8.9 \mathrm{~Hz}, \mathrm{~J}=6.2 \mathrm{~Hz}), 3.64$ (1H, ddd, $\mathrm{J}=10.5 \mathrm{~Hz}, \mathrm{~J}=9.6 \mathrm{~Hz}, \mathrm{~J}=4.4 \mathrm{~Hz}), 3.67(3 \mathrm{H}, \mathrm{s}), 3.78(1 \mathrm{H}, \mathrm{br} \mathrm{s}), 4.24(1 \mathrm{H}, \mathrm{tq}, \mathrm{J}=6.4 \mathrm{~Hz}, \mathrm{~J}=6.3$ $\mathrm{Hz}), 4.55(1 \mathrm{H}, \mathrm{s}) ;{ }^{13} \mathrm{C}\left\{{ }^{1} \mathrm{H}\right\} \mathrm{NMR}\left(100 \mathrm{MHz}, \mathrm{CD}_{3} \mathrm{OD}\right): \delta(\mathrm{ppm})-4.8(2),-4.5,-3.8,18.5,18.8,18.9$, $19.1,26.26,37.8,43.2,52.1,69.5,70.0,71.1,71.3,97.4,173.5$.

Synthesis of (3R)-[(2,4-di-O-tert-butyldimethylsilyloxy-3,6-dideoxy-a-L-arabino-hexopyranosyl)oxy]-butanoic acid (20).<smiles>COC(=O)C[C@H](C)O[C@H]1O[C@H](C)[C@@H](O[13CH3])C[C@H]1O[SbH3]</smiles>
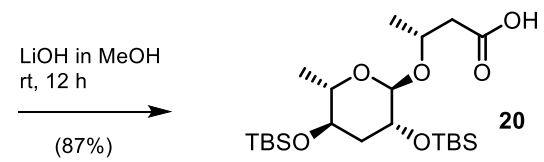

A solution of $10 \mathrm{mg}(3 R)$-methyl 3-[(2,4-di-O-tert-butyldimethylsilyloxy-3,6-dideoxy-a-L-arabinohexopyranosyl)oxy]-butanoate $(21 \mu \mathrm{mol})$ was dissolved in $500 \mathrm{~mL}$ methanol and treated with 15 $\mu \mathrm{L}$ of a saturated aqueous lithium hydroxide solution. The mixture was stirred for $12 \mathrm{~h}$ at room temperature, the $\mathrm{pH}$ adjusted to 6-7 with acetic acid, and the solution concentrated under reduced pressure. Column chromatography on silica gel using $5 \%$ methanol in dichloromethane with $0.5 \%$ acetic acid afforded $8 \mathrm{mg}(3 R)$-[(2,4-di-O-tert-butyldimethylsilyloxy-3,6-dideoxy-a-L-arabinohexopyranosyl)oxy]-buta-noic acid $\left(20,17.3 \mu \mathrm{mol}, 87 \%\right.$ yield) as a colorless oil. ${ }^{1} \mathrm{H}$ NMR (400 $\left.\mathrm{MHz}, \mathrm{CD}_{3} \mathrm{OD}\right): \delta(\mathrm{ppm}) 0.08(12 \mathrm{H}, \mathrm{m}), 0.89(9 \mathrm{H}, \mathrm{s}), 0.92(9 \mathrm{H}, \mathrm{s}), 1.17(3 \mathrm{H}, \mathrm{d}, \mathrm{J}=4.7 \mathrm{~Hz}), 1.19$ $(3 \mathrm{H}, \mathrm{d}, \mathrm{J}=5.1 \mathrm{~Hz}), 1.80(2 \mathrm{H}, \mathrm{m}), 2.44(1 \mathrm{H}, \mathrm{m}), 2.50(1 \mathrm{H}, \mathrm{m}), 3.66(2 \mathrm{H}, \mathrm{m}), 3.80(1 \mathrm{H}, \mathrm{br} \mathrm{s}), 4.24$ $(1 \mathrm{H}, \mathrm{tq}, \mathrm{J}=10.4 \mathrm{~Hz}, \mathrm{~J}=6.2 \mathrm{~Hz}), 4.56(1 \mathrm{H}, \mathrm{s}) ;{ }^{13} \mathrm{C}\left\{{ }^{1} \mathrm{H}\right\} \mathrm{NMR}\left(100 \mathrm{MHz}, \mathrm{CD}_{3} \mathrm{OD}\right): \delta(\mathrm{ppm})-4.81$, $-4.79,-4.4,-3.9,18.5,18.8,18.9,19.2,26.3,26.29$ (3), 37.96 (3), 43.4, 69.8, 70.2, 71.3, 71.5, 97.8, 175.2; ESI-HRMS: obsd. $m / z=461.2768[\mathrm{M}-\mathrm{H}]$; , calcd. $m / z=461.2760$ for $\mathrm{C}_{22} \mathrm{H}_{45} \mathrm{O}_{6} \mathrm{Si}_{2}, \Delta$ $=1.7 \mathrm{ppm}$.

Synthesis of (3'R,3S,4S,7S)-7-[(3'-[(2,4-di-O-tert-butyldimethylsilyloxy-3',6"'-dideoxy-a-Larabino-hexopyranosyl)-oxy]-butanoyl)oxy]-3,4-cis-methylene-1-O-tert-butyldimethylsilyloxy decane (21).
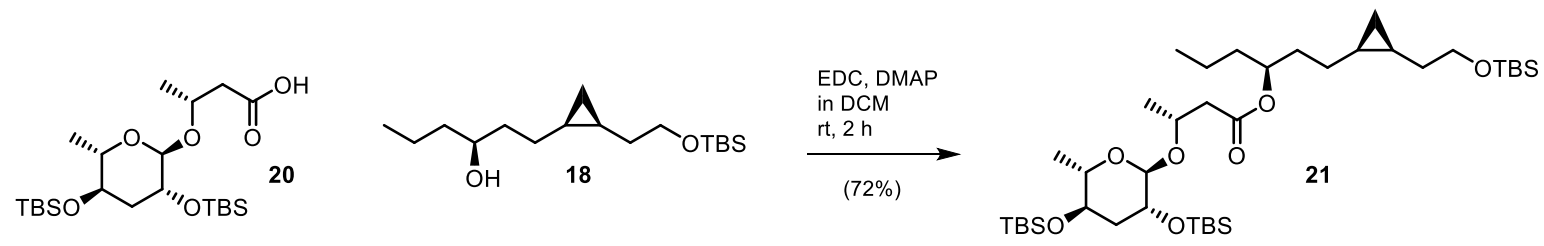

A solution of $3.0 \mathrm{mg}$ (3R)-[(2,4-di-O-tert-butyldimethylsilyloxy-3,6-dideoxy- $\alpha$-L-arabinohexopyranosyl)oxy]-butanoic acid (20) $(6.5 \mu \mathrm{mol})$ in $500 \mu \mathrm{L}$ dry dichloromethane was treated with $2 \mathrm{mg}$ 4-dimethylaminopyridine (DMAP, $16.4 \mu \mathrm{mol}$ ) and $2 \mathrm{mg} 3$-(ethyliminomethylene-amino)- $N, N$ dimethylpropan-1-amine hydrochloride (EDC, $10.4 \mu \mathrm{mol})$. After stirring for $10 \mathrm{~min}, 2.2 \mathrm{mg}$ of 
(3S,4S,7S)-1-O-tert-butyldimethylsilyloxy-cis-3,4-methylenedecan-7-ol (18, $7.3 \mu \mathrm{mol})$ in $300 \mu \mathrm{L}$ dry dichloromethane was added. After stirring for $2 \mathrm{~h}$, the reaction mixture was concentrated under reduced pressure. Column chromatography on silica gel using $5 \%$ ethyl acetate in hexane (v/v) afforded $2.6 \mathrm{mg}$ (3'R,3S,4S,7S)-7-[(3'-[(2,4-di-O-tert-butyldimethylsilyloxy-3",6"'-dideoxy- $\alpha$ L-arabino-hexopyranosyl)-oxy]-butanoyl)oxy]-3,4-cis-methylene-1-O-tert-butyldimethylsilyloxy decane $\left(21,3.5 \mu \mathrm{mol}, 54 \%\right.$ yield) as a colorless oil. ${ }^{1} \mathrm{H} \mathrm{NMR}\left(400 \mathrm{MHz}, \mathrm{CDCl}_{3}\right): \delta(\mathrm{ppm})-0.28$ $(1 \mathrm{H}, \mathrm{m}), 0.05(18 \mathrm{H}, \mathrm{m}), 0.60(1 \mathrm{H}, \mathrm{m}), 0.71(2 \mathrm{H}, \mathrm{m}), 0.87(9 \mathrm{H}, \mathrm{s}), 0.88(9 \mathrm{H}, \mathrm{s}), 0.90(3 \mathrm{H}, \mathrm{t}, \mathrm{J}=$ $3.9 \mathrm{~Hz}) 0.90(9 \mathrm{H}, \mathrm{s}), 1.18(3 \mathrm{H}, \mathrm{d}, \mathrm{J}=5.7 \mathrm{~Hz}), 1.19(3 \mathrm{H}, \mathrm{d}, \mathrm{J}=5.3 \mathrm{~Hz}), 1.33(6 \mathrm{H}, \mathrm{m}), 1.51(2 \mathrm{H}$, $\mathrm{m}), 1.64(4 \mathrm{H}, \mathrm{m}), 1.75(2 \mathrm{H}, \mathrm{m}), 2.40(1 \mathrm{H}, \mathrm{m}), 2.62(1 \mathrm{H}, \mathrm{m}), 3.60-3.67(4 \mathrm{H}, \mathrm{m}) 3.75(1 \mathrm{H}, \mathrm{s} \mathrm{br})$, $4.25(1 \mathrm{H}, \mathrm{m}), 4.56(1 \mathrm{H}, \mathrm{s}), 4.93(1 \mathrm{H}, \mathrm{m}) ;{ }^{13} \mathrm{C}$ NMR $\left\{{ }^{1} \mathrm{H}\right\}\left(100 \mathrm{MHz}, \mathrm{CDCl}_{3}\right): \delta(\mathrm{ppm})-5.09(2)$, $-4.80,-4.71,-4.54,-4.06,1.17,10.8,12.6,14.2,15.2,18.1,18.2,18.3,18.6,18.7,19.0,24.8$, 25.93 (3), 25.95 (3), 26.16 (3), 32.2, 34.6, 36.3, 37.0, 42.8, 68.2, 68.9, 70.1, 70.3, 74.2, 96.8, 171.2; ESI-HRMS: obsd. $m / z=745.5294\left[\mathrm{M}+\mathrm{H}^{+}\right.$, calcd. $m / z=745.5285$ for $\mathrm{C}_{39} \mathrm{H}_{81} \mathrm{O}_{7} \mathrm{Si}_{3}, \Delta=$ $1.2 \mathrm{ppm}$.

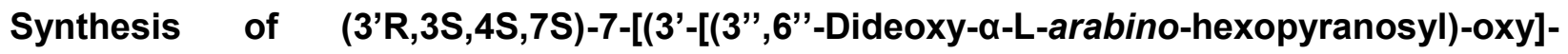
butanoyl)oxy]-3,4-cis-methylene-1-decanol (22).
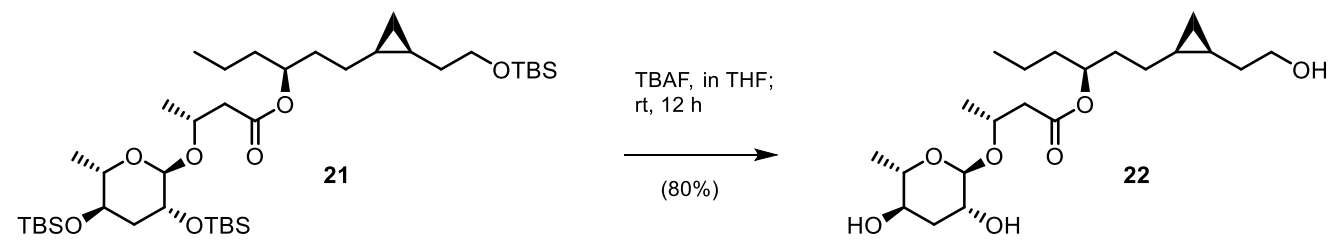

A solution of $1.0 \mathrm{mg}\left(3^{\prime} R, 3 S, 4 S, 7 S\right)-7-\left[\left(3^{\prime}-[(2,4-d i-O-t e r t-b u t y l d i m e t h y l s i l y l o x y-3 ", 6\right.\right.$ ''-dideoxy-a-Larabino-hexopyranosyl)-oxy]-butan-oyl)oxy]-3,4-cis-methylene-1-O-tert-butyldimethylsilyloxy decane $(\mathbf{2 1}, 1.3 \mu \mathrm{mol})$ in $500 \mu \mathrm{L}$ dry tetrahydrofuran was treated with $2 \mu \mathrm{L} 1.0 \mathrm{M}$ tetrabutyl ammonium fluoride (TBAF) solution in tetrahydrofuran $(2 \mu \mathrm{mol})$. After stirring for $12 \mathrm{~h}$ the solution was concentrated under reduced pressure. Column chromatography using $5 \%$ methanol in dichloromethane (v/v) afforded $0.4 \mathrm{mg} \quad\left(3^{\prime} R, 3 S, 4 S, 7 S\right)-7-\left[\left(3^{\prime}-\left[\left(3^{\prime \prime}, 6^{\prime \prime}\right.\right.\right.\right.$-dideoxy- $\alpha$-L-arabinohexopyranosyl)-oxy]-butanoyl)oxy]-3,4-cis-methylene-1-decanol (22, $1.0 \mu \mathrm{mol}$, yield $80 \%)$ as a colorless oil (that still contained some tetrabutylammonium fragments as impurity). ${ }^{1} \mathrm{H}$ NMR (400 $\left.\mathrm{MHz}, \mathrm{CD}_{3} \mathrm{OD}\right): \delta(\mathrm{ppm})-0.24(1 \mathrm{H}, \mathrm{m}), 0.65(1 \mathrm{H}, \mathrm{m}), 0.77(2 \mathrm{H}, \mathrm{m}), 0.93(3 \mathrm{H}, \mathrm{t}, \mathrm{J}=7.3 \mathrm{~Hz}), 1.21$ $(3 \mathrm{H}, \mathrm{d}, \mathrm{J}=6.5 \mathrm{~Hz}), 1.22(3 \mathrm{H}, \mathrm{d}, \mathrm{J}=6.5 \mathrm{~Hz}), 1.24(2 \mathrm{H}, \mathrm{m}), 1.26(2 \mathrm{H}, \mathrm{m}), 1.36(2 \mathrm{H}, \mathrm{m}), 1.55(2 \mathrm{H}$, $\mathrm{m}), 1.68(2 \mathrm{H}, \mathrm{m}), 1.73(1 \mathrm{H}, \mathrm{m}), 1.92(1 \mathrm{H}, \mathrm{dt}, \mathrm{J}=13.3 \mathrm{~Hz}, \mathrm{~J}=3.8 \mathrm{~Hz}), 2.52(2 \mathrm{H}, \mathrm{m}), 3.51(1 \mathrm{H}$, ddd, $\mathrm{J}=4.9 \mathrm{~Hz}, \mathrm{~J}=9.2 \mathrm{~Hz}, \mathrm{~J}=11.0 \mathrm{~Hz}), 3.61(1 \mathrm{H}, \mathrm{m}), 3.71(1 \mathrm{H}, \mathrm{s} \mathrm{br}), 4.23(1 \mathrm{H}, \mathrm{m}), 4.67(1 \mathrm{H}, \mathrm{s}), 4.92$ $(1 \mathrm{H}, \mathrm{m}) ;{ }^{13} \mathrm{C}$ NMR $\left(100 \mathrm{MHz}, \mathrm{CD}_{3} \mathrm{OD}\right): \delta$ (ppm) $11.1,13.4,13.8,15.9,17.7,19.0,25.6,32.6,32.7$, 35.8, 37.0, 43.4, 67.9, 69.5, 69.5, 71.1, 75.3, 98.1; ESI-HRMS: obsd. $\mathrm{m} / \mathrm{z}=425.2517[\mathrm{M}+\mathrm{Na}]^{+}$, calcd. $m / z=425.2510$ for $\mathrm{C}_{21} \mathrm{H}_{38} \mathrm{NaO}_{7}, \Delta=1.6 \mathrm{ppm}$. 
Synthesis of $\quad\left(3^{\prime} R, 3 S, 4 S, 7 S\right)-7-\left[\left(3^{\prime}-\left[\left(3^{\prime \prime}, 6^{\prime \prime}-\right.\right.\right.\right.$ Dideoxy-a-L-arabino-hexopyranosyl)-oxy]butanoyl)oxy]-3,4-cis-methylenedecanoic acid (fasc\#1, 3).
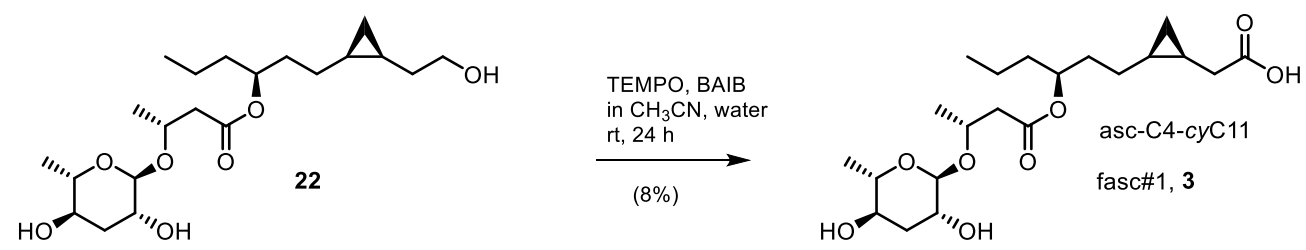

A solution of $0.5 \mathrm{mg}\left(3^{\prime} R, 3 S, 4 S, 7 S\right)-7-\left[\left(3^{\prime}-\left[\left(3^{\prime \prime}, 6\right.\right.\right.\right.$ '-dideoxy- $\alpha$-L-arabino-hexopyranosyl)-oxy]butanoyl)oxy]-3,4-cis-methylene-1-decanol $(22,1.2 \mu \mathrm{mol})$ in $400 \mu \mathrm{L}$ aqueous $50 \%$ acetonitrile was treated with $0.2 \mathrm{mg}(2,2,6,6$-tetramethylpiperidin-1-yl)oxyl (TEMPO) $(1.3 \mu \mathrm{mol})$ and $1.2 \mathrm{mg}$ bis(acetoxy)-iodobenzene (BAIB, $3.6 \mathrm{mmol}$ ) in $50 \mu \mathrm{l}$ water. After stirring for $12 \mathrm{~h}$ another $0.2 \mathrm{mg}$ TEMPO $(1.3 \mu \mathrm{mol})$ and $1.2 \mathrm{mg}$ BAIB $(3.6 \mathrm{mmol})$ in $50 \mu \mathrm{l}$ were added. After stirring for $12 \mathrm{~h}$ the solution was concentrated under reduced pressure. The residue was fractionated by column chromatography on silica gel using $5 \%$ methanol in dichloromethane, followed by solid phase extraction on $100 \mathrm{mg} \mathrm{RP}-\mathrm{C} 18 \mathrm{ec}$ (Chromabond, Macherey Nagel) using a stepwise increase of methanol in water as eluent ( 0 to $100 \%$ in $10 \%$ steps), followed by preparative HPLC to afford approximately $40 \mu \mathrm{g} \quad\left(3^{\prime} R, 3 S, 4 S, 7 S\right)-7-\left[\left(3^{\prime}-\left[\left(3^{\prime \prime}, 6^{\prime \prime}\right.\right.\right.\right.$-dideoxy- $\alpha$-L-arabino-hexopyranosyl)-oxy]butanoyl)oxy]-3,4-cis-methylenedecanoic acid $(3,95 \mu \mathrm{mol}, 8 \%$ yield) identical to the natural product isolated from $C$. remanei based on comparison of the chromatographic behavior on reverse phase C18 HPLC (Figure S3), mass spectrometric fragmentation upon HR-MS (Figure S4a), and HR-MSMS (Figure S4b), as well as comparison of NMR data (Figures S5a \& S5b). For ${ }^{1} \mathrm{H}$ and ${ }^{13} \mathrm{C}$ NMR data see Table S3; ESI-HRMS: obsd. $\mathrm{m} / \mathrm{z}=425.2517[\mathrm{M}+\mathrm{H}]^{+}$, calcd. $\mathrm{m} / \mathrm{z}=$ 425.2510 for $\mathrm{C}_{21} \mathrm{H}_{38} \mathrm{NaO}_{7}, \Delta=1.6 \mathrm{ppm}$.

Synthesis of methyl palmitoleate.
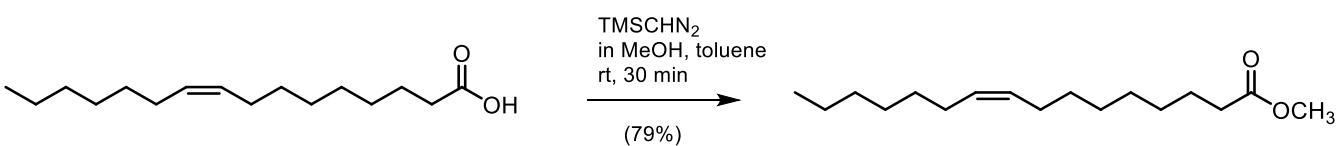

A solution of $509 \mathrm{mg}$ palmitoleic acid (16:1(n7); $2 \mathrm{mmol})$ in $1 \mathrm{ml}$ toluene and $1 \mathrm{ml}$ methanol at 0 ${ }^{\circ} \mathrm{C}$ was treated with $2 \mathrm{ml}$ of a $2 \mathrm{M}$ trimethylsilyl diazomethane solution $(4 \mathrm{mmol})$ in diethyl ether. After $30 \mathrm{~min}$, excess reagent was quenched by addition of acetic acid and the solution was concentrated under reduced pressure. Column chromatography on silica gel (hexanedichloromethane $7: 1)$ afforded $421 \mathrm{mg}$ methyl palmitoleate as a colorless oil $(1.57 \mathrm{mmol}, 79 \%$ yield). ${ }^{1} \mathrm{H}$ NMR $\left(400 \mathrm{MHz}, \mathrm{CDCl}_{3}\right): \delta(\mathrm{ppm}) 0.87(3 \mathrm{H}, t, \mathrm{~J}=7.0 \mathrm{~Hz}), 1.30(16 \mathrm{H}, m), 1.61(2 \mathrm{H}, m)$, $2.00(4 \mathrm{H}, m), 2.28(2 \mathrm{H}, t, \mathrm{~J}=7.6 \mathrm{~Hz}), 3.64(3 \mathrm{H}, s), 5.33(2 \mathrm{H}, m) ;{ }^{13} \mathrm{C}\left\{{ }^{1} \mathrm{H}\right\} \mathrm{NMR}\left(100 \mathrm{MHz}, \mathrm{CDCl}_{3}\right)$ : $\delta$ (ppm) 14.2, 22.8, 25.1, 27.3, 27.4, 29.1, 29.20, 29.25, 29.26, 29.79, 29.85, 31.9, 34.2, 51.5, 129.8, 130.1, 174.3; GC-EIMS $(70 \mathrm{eV}) \mathrm{m} / \mathrm{z}=236\left[\mathrm{M}-\mathrm{HOCH}_{3}\right]$.

\section{Synthesis of ( \pm )-methyl cis-9,10-methylenehexadecanoate.}

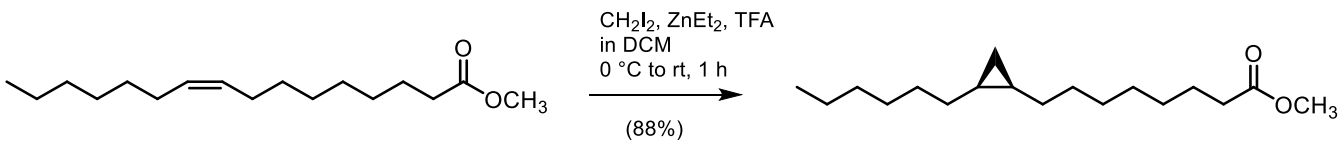


To $4 \mathrm{ml}$ dry dichloromethane under argon was added $4 \mathrm{ml} 1.0 \mathrm{M}$ diethylzinc in $n$-hexane (4 mmol). The solution was cooled to $0{ }^{\circ} \mathrm{C}$ and a solution of $308 \mu \mathrm{l}$ trifluoracetic acid $(4 \mathrm{mmol})$ in $2 \mathrm{ml}$ dichloromethane was added drop wise over the course of $10 \mathrm{~min} .{ }^{[8]}$ After stirring for $20 \mathrm{~min}$ a solution of $322 \mu \mathrm{l}$ diiodomethane $(4 \mathrm{mmol})$ in $2 \mathrm{ml}$ dichloromethane was added. After stirring for $20 \mathrm{~min}$ a solution of $415 \mathrm{mg}$ methyl palmitoleate $(1.54 \mathrm{mmol})$ in $2 \mathrm{ml}$ dichloromethane was added and the ice bath removed. After stirring for 60 min the reaction mixture was quenched with $10 \mathrm{ml}$ $0.1 \mathrm{M}$ hydrochloric acid and the layers separated. The aqueous layer was extracted with $2 \times 10$ $\mathrm{ml}$ dichloromethane. Combined organic layers were washed with $10 \%$ aqueous $\mathrm{NaHCO}_{3}, \mathrm{H}_{2} \mathrm{O}$, brine, and dried over $\mathrm{Na}_{2} \mathrm{SO}_{4}$, filtered, and concentrated in under reduced pressure to afford racemic $385 \mathrm{mg}$ methyl cis-9,10-methyleneoctadecanoate $(1.36 \mathrm{mmol}, 88 \%$ yield $)$ as a colorless oil. ${ }^{1} \mathrm{H}$ NMR $\left(400 \mathrm{MHz} \mathrm{CDCl}_{3}\right): \delta(\mathrm{ppm})-0.34(1 \mathrm{H}, \mathrm{m}), 0.55(1 \mathrm{H}, \mathrm{m}), 0.64(2 \mathrm{H}, \mathrm{m}), 0.88(3 \mathrm{H}, \mathrm{t}, \mathrm{J}$ $=6.9 \mathrm{~Hz}), 1.13(2 \mathrm{H}, \mathrm{m}), 1.27-1.36(18 \mathrm{H}, \mathrm{m}), 1.62(2 \mathrm{H}, \mathrm{m}), 2.30(2 \mathrm{H}, \mathrm{t}, \mathrm{J}=7.6 \mathrm{~Hz}), 3.66(3 \mathrm{H}, \mathrm{s}$, $\left.\mathrm{OCH}_{3}\right) ;{ }^{13} \mathrm{C}\left\{{ }^{1} \mathrm{H}\right\}$ NMR $\left(100 \mathrm{MHz}, \mathrm{CDCl}_{3}\right): \delta(\mathrm{ppm}) 10.9,14.1,15.8,15.8,22.7,25.0,28.7,28.8$, 29.2, 29.4, 29.4, 29.5, 30.2, 30.2, 32.0, 34.1, 51.3, 174.1; GC-EIMS (70 eV) m/z = 250 [M $\mathrm{HOCH}_{3}$.

\section{Synthesis of ( \pm )-cis-9,10-methylenehexadecanoic acid (25).}

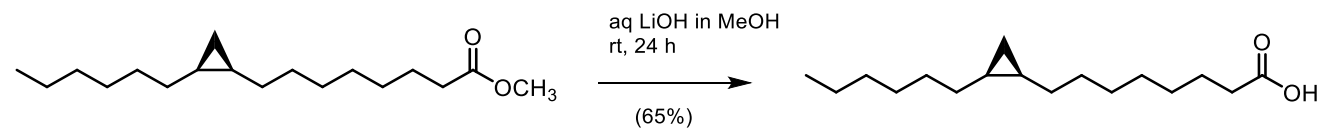

A solution of $385 \mathrm{mg}$ methyl cis-9,10-methyleneoctadecanoate $(1.36 \mathrm{mmol})$ in $5 \mathrm{ml}$ tetrahydrofuran was treated with $325 \mathrm{mg}$ lithium hydroxide $(13.6 \mathrm{mmol})$ and $1 \mathrm{ml}_{2} \mathrm{O}$ and stirred at RT for $24 \mathrm{~h}$ during which a white precipitate formed after several hours. The mixture was acidified by addition of $1.2 \mathrm{ml}$ acetic acid $(21 \mathrm{mmol})$ and concentrated under reduced pressure. The residue was extracted with dichloromethane and the organic phase dried over $\mathrm{Na}_{2} \mathrm{SO}_{4}$, filtered, and concentrated to afford $239 \mathrm{mg}$ cis-9,10-methyleneoctadecanoic acid $(0.89 \mathrm{mmol}$, $65 \%)$ as a white solid. ${ }^{1} \mathrm{H}$ NMR $\left(400 \mathrm{MHz} \mathrm{CDCl}_{3}\right): \delta(\mathrm{ppm})-0.33(1 \mathrm{H}, \mathrm{m}), 0.56(1 \mathrm{H}, \mathrm{ddd}, \mathrm{J}=3.9$ $\mathrm{Hz}, \mathrm{J}=7.6 \mathrm{~Hz}, \mathrm{~J}=8.6 \mathrm{~Hz}), 0.64(2 \mathrm{H}, \mathrm{m}), 0.89(3 \mathrm{H}, \mathrm{t}, \mathrm{J}=7.0 \mathrm{~Hz}), 1.14(2 \mathrm{H}, \mathrm{m}), 1.25-1.41(18 \mathrm{H}$, $\mathrm{m}), 1.63(2 \mathrm{H}, \mathrm{m}), 2.34(2 \mathrm{H}, \mathrm{t}, 7.3 \mathrm{~Hz}), 11.6(1 \mathrm{H}, \mathrm{s}) ;{ }^{13} \mathrm{C}\left\{{ }^{1} \mathrm{H}\right\} \mathrm{NMR}\left(100 \mathrm{MHz}, \mathrm{CDCl}_{3}\right): \delta(\mathrm{ppm}) 11.1$, 14.2, 15.89, 15.93, 22.8, 24.9, 28.8, 28.9, 29.3, 29.46, 29.51, 29.59, 30.2, 30.3, 32.1, 34.4, 180.8; ESI-HRMS: obsd. $m / z 267.2338[\mathrm{M}-\mathrm{H}]^{-}$, calcd. $\mathrm{m} / \mathrm{z} 267.2330$ for $\mathrm{C}_{17} \mathrm{H}_{31} \mathrm{O}_{2} ; \Delta=3.0$ ppm.

Preparation of potassium ( $\mathbf{\pm}$ )-cis-9,10-methylenehexadecanoate. A solution of $134.2 \mathrm{mg}$ cis9,10-methylenehexadecanoic acid (cyC17, 25, $0.5 \mathrm{mmol}$ ) in $2.5 \mathrm{ml}$ ethanol was treated with 500 $\mu \mathrm{l}$ of a $1 \mathrm{M}$ aqueous potassium hydroxide solution. After stirring for $1 \mathrm{~h}$ at RT the solution was concentrated under reduced pressure and the residue dissolved in $10 \mathrm{ml}$ autoclaved water to give a $50 \mathrm{mM}$ stock solution of potassium cis-9,10-methylenehexadecanoate. 


\section{Supporting Tables}

Table S1: $700 \mathrm{MHz}$ NMR data (in $\mathrm{CD}_{3} \mathrm{OD}$ ) of asc-C4-cyC11 (fasc\#1, 3) and asc-C4-C12:1 (fasc\#2, 4) isolated from C. remanei strain PB4641 ( $\delta[\mathrm{ppm}]$ from ${ }^{1} \mathrm{H}$ NMR, dqf-COSY, HSQC, and $\mathrm{HMBC}$ data, multiplicity, $J[\mathrm{~Hz}]$, ${ }^{*}$ chemical shift strongly $\mathrm{pH}$ dependent (Figure $\mathrm{S} 5 \mathrm{c}$ )).

\begin{tabular}{|c|c|c|c|c|}
\hline \multirow[b]{2}{*}{ position } & \multicolumn{2}{|c|}{ asc-C4-cyC11 (fasc\#1, 3) } & \multicolumn{2}{|c|}{ asc-C4-C12:1 (fasc\#2, 4) } \\
\hline & ${ }^{1} \mathrm{H}$ & ${ }^{13} \mathrm{C}$ & ${ }^{1} \mathrm{H}$ & ${ }^{13} \mathrm{C}$ \\
\hline 1 & $4.67 \mathrm{~s}$ & 97.2 & $4.67 s$ & 97.3 \\
\hline 2 & 3.72 s.br & 69.5 & 3.71 s.br & 69.5 \\
\hline $\begin{array}{l}3 a \\
3 b\end{array}$ & $\begin{array}{c}1.74 d d d 13.2,11.5,3.2 \\
1.92 d t 13.5,3.9\end{array}$ & 35.5 & $\begin{array}{c}1.74 \text { ddd } 13.1,11.2,3.5 \\
1.92 d t 13.2,3.7\end{array}$ & 35.7 \\
\hline 4 & 3.51 ddd $11.4,9.7,4.6$ & 68.0 & $3.51 d d d 11.1,9.1,4.2$ & 68.1 \\
\hline 5 & $3.62 d q 9.2,6.5$ & 70.9 & $3.64 d q 9.0,6.3$ & 71.0 \\
\hline 6 & $1.18 d 6.6$ & 17.8 & $1.22 d 6.4$ & 18.4 \\
\hline 1 ' & - & 172.5 & - & 172.5 \\
\hline 2'a & $2.58 d d 15.1,7.3$ & 43.1 & $2.60 d d 15.1,7.0$ & 43.2 \\
\hline 2 'b & $2.49 d d 15.2,5.7$ & & $2.50 d d 15.2,5.9$ & \\
\hline $3^{\prime}$ & $4.23 \mathrm{~m}$ & 69.3 & $4.23 \mathrm{~m}$ & 69.4 \\
\hline 4' & $1.21 d 6.6$ & 18.8 & $1.22 d 6.4$ & 18.4 \\
\hline 1" & - & 181.2 & - & 178.3 \\
\hline 2"a & $2.24^{*} d d 15.5,7.4$ & 37.2 & $2.97 d 7.2$ & 35.9 \\
\hline $2 " b$ & $2.11^{*} d d 15.4,7.7$ & & & \\
\hline 3" & 1.12 ddd $11.1,11.1,5.2$ & 13.5 & $5.62 \mathrm{~m}$ & 125.3 \\
\hline 4" & $0.76 \mathrm{~m}$ & 16.1 & $5.45 \mathrm{~m}$ & 131.2 \\
\hline $5 " a$ & $1.25 \mathrm{~m}$ & 25.5 & $2.10 \mathrm{~m}$ & 24.1 \\
\hline $5 " \mathrm{~b}$ & $1.39 \mathrm{~m}$ & & & \\
\hline 6" & $1.67 \mathrm{~m}$ & 35.2 & $1.63 \mathrm{~m}$ & 34.5 \\
\hline 7" & $4.92 \mathrm{~m}$ & 75.3 & $4.89 \mathrm{~m}$ & 75.2 \\
\hline 8" & $1.55 \mathrm{~m}$ & 37.0 & $1.58 \mathrm{~m}$ & 34.8 \\
\hline 9" & $1.38 \mathrm{~m}$ & 19.7 & $1.34 \mathrm{~m}$ & 25.9 \\
\hline 10" & $0.92 t 7.5$ & 14.0 & $1.58 \mathrm{~m}$ & 32.7 \\
\hline $\begin{array}{l}11 " \mathrm{a} \\
11 " \mathrm{~b}\end{array}$ & $\begin{array}{l}0.67 d t 4.4,8.5 \\
-0.16 \mathrm{~m}\end{array}$ & 11.0 & $1.33 \mathrm{~m}$ & 23.1 \\
\hline $12 "$ & - & - & $0.91 t 7.0$ & 14.1 \\
\hline
\end{tabular}


Table S2: ${ }^{1} \mathrm{H}$ NMR data of $(S)$ - and (R)-MTPA esters of methyl (3S,4S,7S)-7-hydroxy-cis-3,4methylene decanoate (prepared using $(R)$ - and (S)-MTPA chloride, respectively) derived from SELTOCSY, dqf-COSY and HSQC spectra $\left({ }^{1} \mathrm{H}: 700 \mathrm{MHz},{ }^{13} \mathrm{C} 175 \mathrm{MHz}\right.$; in $\mathrm{CDCl}_{3}$ with $6.5 \%$ pyridine- $d_{5}$ ).

\begin{tabular}{|c|c|c|c|}
\hline & $\delta_{\mathrm{H}}(\mathrm{S})-\operatorname{MTPA-X}(\mathbf{1 0 a})$ & $\delta_{\mathrm{H}}(R)-\operatorname{MTPA}-\mathrm{X}(\mathbf{1 0 b})$ & $\Delta \delta^{S R}\left(=\delta_{S}-\delta_{R}\right)$ \\
\hline $\mathrm{OCH}_{3}$ & 3.563 & 3.569 & -0.006 \\
\hline 1 & - & - & - \\
\hline 2 anti & 2.172 & 2.134 & +0.038 \\
\hline $2_{\text {syn }}$ & 2.162 & 2.076 & +0.086 \\
\hline 3 & 1.016 & 0.979 & +0.037 \\
\hline 4 & 0.701 & 0.639 & +0.062 \\
\hline $5_{\text {syn }}$ & 1.063 & 0.907 & +0.156 \\
\hline 5 anti & 1.320 & 1.214 & +0.106 \\
\hline $6_{s y n}$ & 1.674 & 1.582 & +0.092 \\
\hline 6 anti & 1.608 & 1.582 & +0.026 \\
\hline 7 & 5.030 & 5.027 & +0.003 \\
\hline $8_{\text {syn }}$ & 1.491 & 1.573 & -0.082 \\
\hline 8 anti & 1.424 & 1.482 & -0.058 \\
\hline 9 anti & 1.160 & 1.264 & -0.104 \\
\hline $9_{\text {syn }}$ & 1.104 & 1.264 & -0.160 \\
\hline 10 & 0.754 & 0.827 & -0.073 \\
\hline $11_{\text {cis }}$ & -0.264 & -0.339 & +0.075 \\
\hline 11 trans & 0.639 & 0.598 & +0.041 \\
\hline
\end{tabular}


Table S3: Comparison of $700 \mathrm{MHz}$ NMR data (in $\mathrm{CD}_{3} \mathrm{OD}$ ) of synthetic fasc\#1 (3) and the natural product isolated from the exometabolome extract of $C$. remanei PB4641 $\left(\delta[\mathrm{ppm}]\right.$ from ${ }^{1} \mathrm{H}$ NMR, dqf-COSY, HSQC, and HMBC data, multiplicity, $J[\mathrm{~Hz}]$, " chemical shift strongly $\mathrm{pH}$ dependent (Figure S5c)).

\begin{tabular}{|c|c|c|c|c|}
\hline \multirow[b]{2}{*}{ position } & \multicolumn{2}{|c|}{ natural asc-C4-cyC11 (fasc\#1, 3) } & \multicolumn{2}{|c|}{ synthetic asc-C4-cyC11 (fasc\#1, 3) } \\
\hline & ${ }^{1} \mathrm{H}$ & ${ }^{13} \mathrm{C}$ & ${ }^{1} \mathrm{H}$ & ${ }^{13} \mathrm{C}$ \\
\hline 1 & $4.67 \mathrm{~s}$ & 97.2 & $4.66 s$ & 97.1 \\
\hline 2 & 3.72 s.br & 69.5 & 3.71 s.br & 69.3 \\
\hline $3 a$ & $1.74 d d d 13.2,11.5,3.2$ & 35.5 & $1.73 d d d 13.0,11.7,3.0$ & 35.3 \\
\hline $3 b$ & $1.92 d t 13.5,3.9$ & & $1.92 d t 13.2,3.4$ & \\
\hline 4 & $3.51 d d d 11.4,9.7,4.6$ & 68.0 & $3.51 d d d 11.0,9.2,4.6$ & 67.8 \\
\hline 5 & $3.62 d q 9.2,6.5$ & 70.9 & $3.64 d q 9.3,6.2$ & 70.8 \\
\hline 6 & $1.18 d 6.6$ & 17.8 & $1.22 d 6.3$ & 17.7 \\
\hline $1^{\prime}$ & - & 172.5 & - & nd \\
\hline 2'a & $2.58 d d 15.1,7.3$ & 43.1 & $2.58 d d 15.3,7.3$ & 43.0 \\
\hline $2^{\prime} b$ & $2.49 d d 15.2,5.7$ & & $2.47 d d 15.3,6.1$ & \\
\hline 3 ' & $4.23 \mathrm{~m}$ & 69.3 & $4.23 \mathrm{~m}$ & 69.1 \\
\hline 4 & $1.21 d$ & 18.8 & $1.21 d 6.3$ & 18.1 \\
\hline 1" & - & 181.2 & - & nd \\
\hline 2"a & $2.24^{*} d d 15.5,7.4$ & 37.2 & $2.28^{*} d d 15.1,6.8$ & 36.9 \\
\hline 2"b & $2.11^{*} d d 15.4,7.7$ & & $2.13^{*} d d 15.3,7.8$ & \\
\hline 3" & $1.12 d d d 11.1,11.1,5.2$ & 13.5 & $1.12 \mathrm{~m}$ & 13.2 \\
\hline 4" & $0.76 \mathrm{~m}$ & 16.1 & $0.78 \mathrm{~m}$ & 15.7 \\
\hline 5"a & $1.25 \mathrm{~m}$ & 25.5 & $1.25 \mathrm{~m}$ & 25.5 \\
\hline $5 " \mathrm{~b}$ & $1.39 \mathrm{~m}$ & & $1.39 \mathrm{~m}$ & \\
\hline 6" & $1.67 \mathrm{~m}$ & 35.2 & $1.67 \mathrm{~m}$ & 35.0 \\
\hline 7" & $4.92 \mathrm{~m}$ & 75.3 & $4.93 \mathrm{~m}$ & 75.1 \\
\hline 8" & $1.55 \mathrm{~m}$ & 37.0 & $1.55 \mathrm{~m}$ & 36.8 \\
\hline 9" & $1.38 \mathrm{~m}$ & 19.7 & 1.38 & 19.5 \\
\hline $10 "$ & $0.92+7.5$ & 14.0 & $0.92+7.3$ & 13.9 \\
\hline $\begin{array}{l}11 " \mathrm{a} \\
11 " \mathrm{~b}\end{array}$ & $\begin{array}{c}0.67 d t 4.4,8.5 \\
-0.16 m\end{array}$ & 11.0 & $\begin{array}{c}0.67 d t 4.7,8.6 \\
-0.15 m\end{array}$ & 10.8 \\
\hline
\end{tabular}


Table S4: $700 \mathrm{MHz}$ NMR data of fasc\#4 (asc-C4-C8, 7) and fasc\#5 (asc-C4-C6) (in $\mathrm{CD}_{3} \mathrm{OD}$ ) isolated from $C$. latens strain VX88 $\left(\delta[\mathrm{ppm}]\right.$ from ${ }^{1} \mathrm{H}$ NMR, dqf-COSY, HSQC, and HMBC data multiplicity, $J[\mathrm{~Hz}]$, ${ }^{*}$ chemical shift strongly $\mathrm{pH}$ dependent).

\begin{tabular}{|c|c|c|c|c|}
\hline \multirow[b]{2}{*}{ position } & \multicolumn{2}{|c|}{ asc-C4-C8 (fasc\#4) } & \multicolumn{2}{|c|}{ asc-C4-C6 (fasc\#5) } \\
\hline & ${ }^{1} \mathrm{H}$ & ${ }^{13} \mathrm{C}$ & ${ }^{1} \mathrm{H}$ & ${ }^{13} \mathrm{C}$ \\
\hline 1 & $4.66 s$ & 97.2 & $4.67 s$ & 97.2 \\
\hline 2 & 3.71 s.br & 69.6 & 3.72 s.br & 69.5 \\
\hline $\begin{array}{l}3 a \\
3 b\end{array}$ & $\begin{array}{c}1.75 d d d 13.2,11.5,3.2 \\
1.92 d t 13.5,3.9\end{array}$ & 35.7 & $\begin{array}{c}1.76 d d d 13.4,11.6,3.0 \\
1.95 d t 13.2,3.6\end{array}$ & 35.6 \\
\hline 4 & 3.49 ddd 11.4, 9.7, 4.6 & 68.1 & $3.52 d d d 11.1,9.6,4.5$ & 68.1 \\
\hline 5 & $3.64 d q 9.2,6.5$ & 71.0 & $3.63 d q 9.5,6.1$ & 70.9 \\
\hline 6 & $1.22 d 6.4$ & 17.8 & $1.22 d \mathrm{~J} 6.3$ & 17.8 \\
\hline $1^{\prime}$ & - & 172.1 & - & 172.1 \\
\hline $\begin{array}{l}2 ' a \\
\text { 2'b }\end{array}$ & $\begin{array}{l}2.58 d d 15.1,7.3 \\
2.45 d d 15.2,5.7\end{array}$ & 43.2 & $\begin{array}{l}2.58 d d 15.1,7.0 \\
2.46 d d 15.2,5.9\end{array}$ & 43.2 \\
\hline 3 & $4.22 \mathrm{~m}$ & 69.3 & $4.22 \mathrm{~m}$ & 69.2 \\
\hline $4^{\prime}$ & $1.21 d 6.2 \mathrm{~Hz}$ & 19.0 & $1.20 d \mathrm{~J} 6.4 \mathrm{~Hz}$ & 18.9 \\
\hline $1 "$ & - & 178.1 & - & 175.4 \\
\hline $\begin{array}{l}\text { 2"a } \\
2 " b\end{array}$ & $\begin{array}{l}2.49^{*} d d 15.5,7.4 \\
2.38^{*} d d 15.4,6.3\end{array}$ & 43.3 & $2.53^{*} d 7.2$ & 43.5 \\
\hline $3 "$ & $5.26 \mathrm{~m}$ & 74.0 & $5.25 \mathrm{~m}$ & 72.5 \\
\hline 4" & $1.63 \mathrm{~m}$ & 35.0 & $1.62 m$ & 37.3 \\
\hline $5 "$ & $1.35 \mathrm{~m}$ & 25.8 & $1.34 \mathrm{~m}$ & 26.2 \\
\hline 6" & $1.31 \mathrm{~m}$ & 32.6 & $0.94 t 7.3$ & 14.2 \\
\hline 7" & $1.33 \mathrm{~m}$ & 23.3 & --- & --- \\
\hline 8" & $0.90 t 6.9$ & 14.1 & --- & --- \\
\hline
\end{tabular}


Table S5a: HPLC-ESI-(-)-MS ${ }^{n}$ data of saturated fatty acid ascarosides from C. remanei and C. latens. See Figure S6a for structures and Figures $\mathrm{S} 9 \mathrm{a}-\mathrm{S} 9 \mathrm{~h}$ for $\mathrm{MS}^{\mathrm{n}}$ spectra.

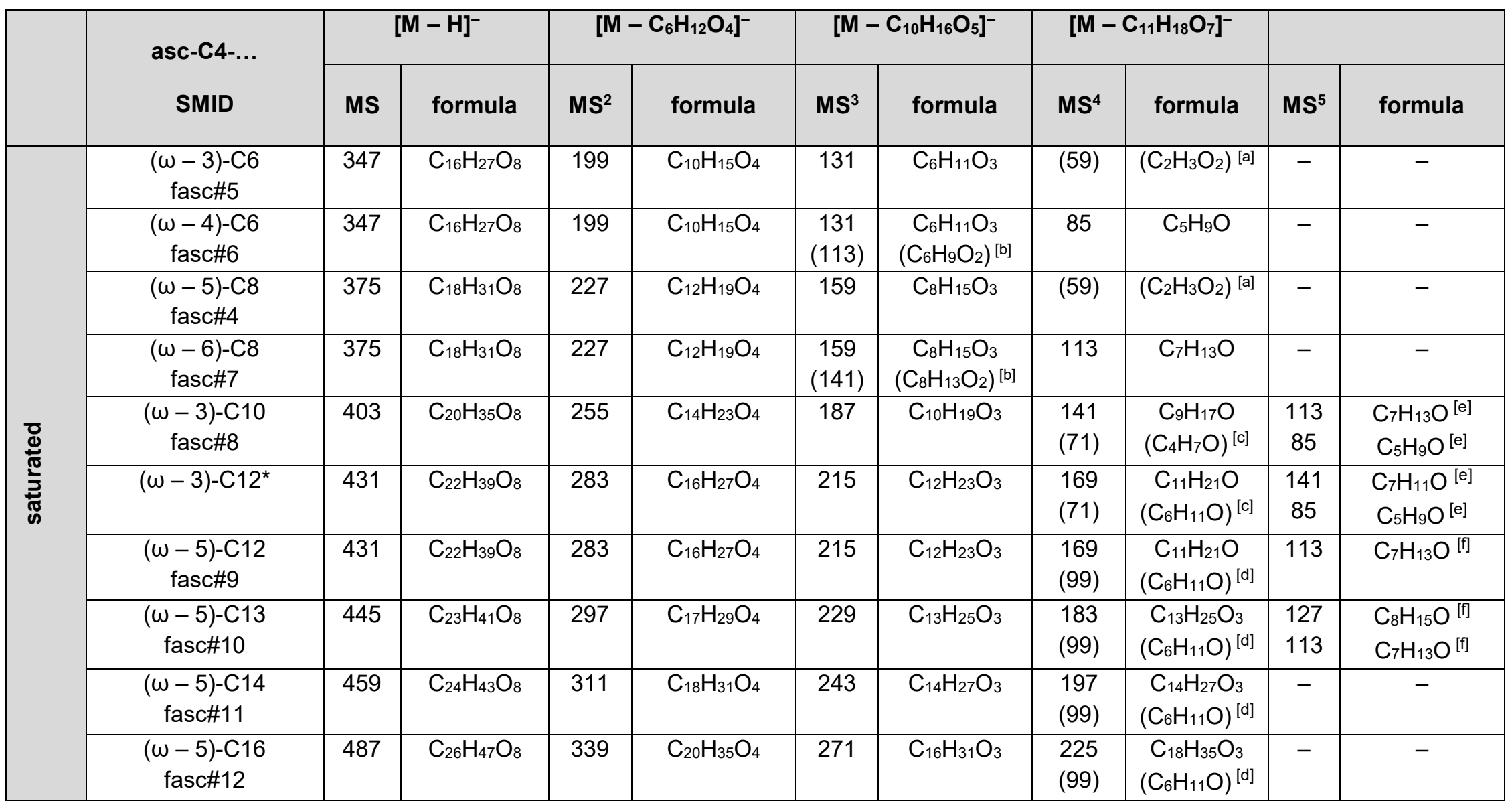

[a] dominating acetate fragment at $\mathrm{m} / \mathrm{z} 59$ indicates 3-hydroxyfatty acids (confirmed by NMR analysis); [b] dominating loss of water indicates 2-hydroxyfatty acids; [c] MS ${ }^{4}$ fragment ion at $m / z 71$ indicates ( $\left.\omega-3\right)$-linkage; [d] $\mathrm{MS}^{4}$ fragment ion at $m / z 99$ indicates $(\omega-$ 5)-linkage; [e] MS ${ }^{5}$ fragment ions indicate $(\omega-3)$-linkage; [f] $\mathrm{MS}^{5}$ fragment ions indicate $(\omega-5)$-linkage; * semisynthetic compound from the hydrogenation of $(\omega-3)$-linked asc-C4-C12:2 (fasc\#18). 
Table S5b: HPLC-ESI-(-)-MS ${ }^{n}$ data of unsaturated and cyclopropyl fatty acid ascarosides from C. remanei and C. latens. See Figures S6b - S6c for structures and Figures S9i - S9s for $\mathrm{MS}^{\mathrm{n}}$ spectra.

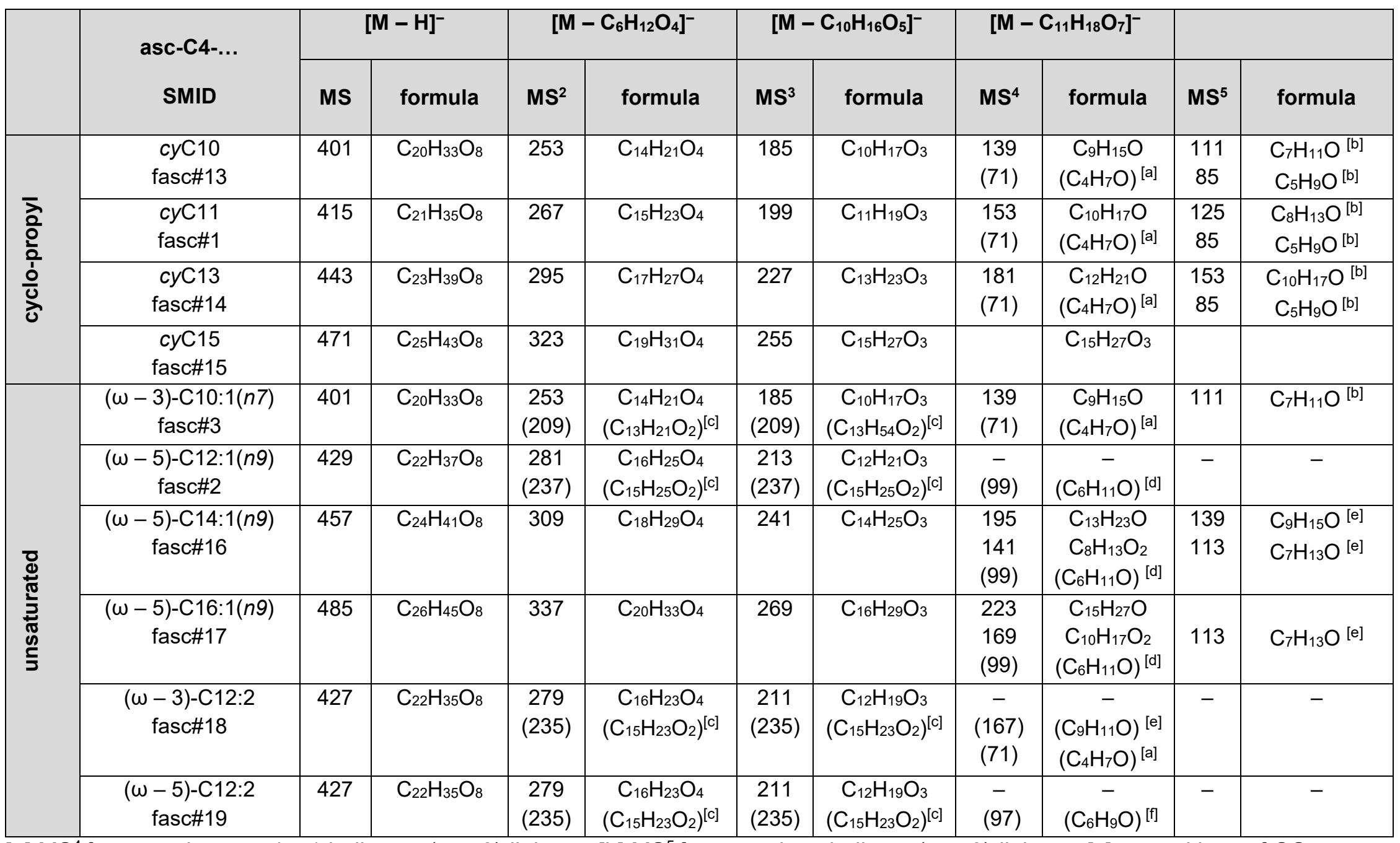

[a] $\mathrm{MS}^{4}$ fragment ion at $\mathrm{m} / \mathrm{z} 71$ indicates $(\omega-3)$-linkage; [b] MS ${ }^{5}$ fragment ions indicate $(\omega-3)$-linkage; [c] neutral loss of $\mathrm{CO}_{2}$ indicates $\Delta^{3}$ double bond; [d] MS ${ }^{4}$ fragment ion at $\mathrm{m} / \mathrm{z} 99$ indicates $(\omega-5)$-linkage; [f] $\mathrm{MS}^{4}$ fragment ion at $\mathrm{m} / \mathrm{z} 97$ indicates $(\omega-5)$ linkage with unsaturated pentenyl chain; $[\mathrm{e}] \mathrm{MS}^{5}$ fragment ions indicates $(\omega-5)$-linkage. 
Table S6: HPLC-ESI-(-)-HRMS-MS/MS data of fatty acid ascarosides from C. remanei and C. latens. See figures S6a-c for structures.

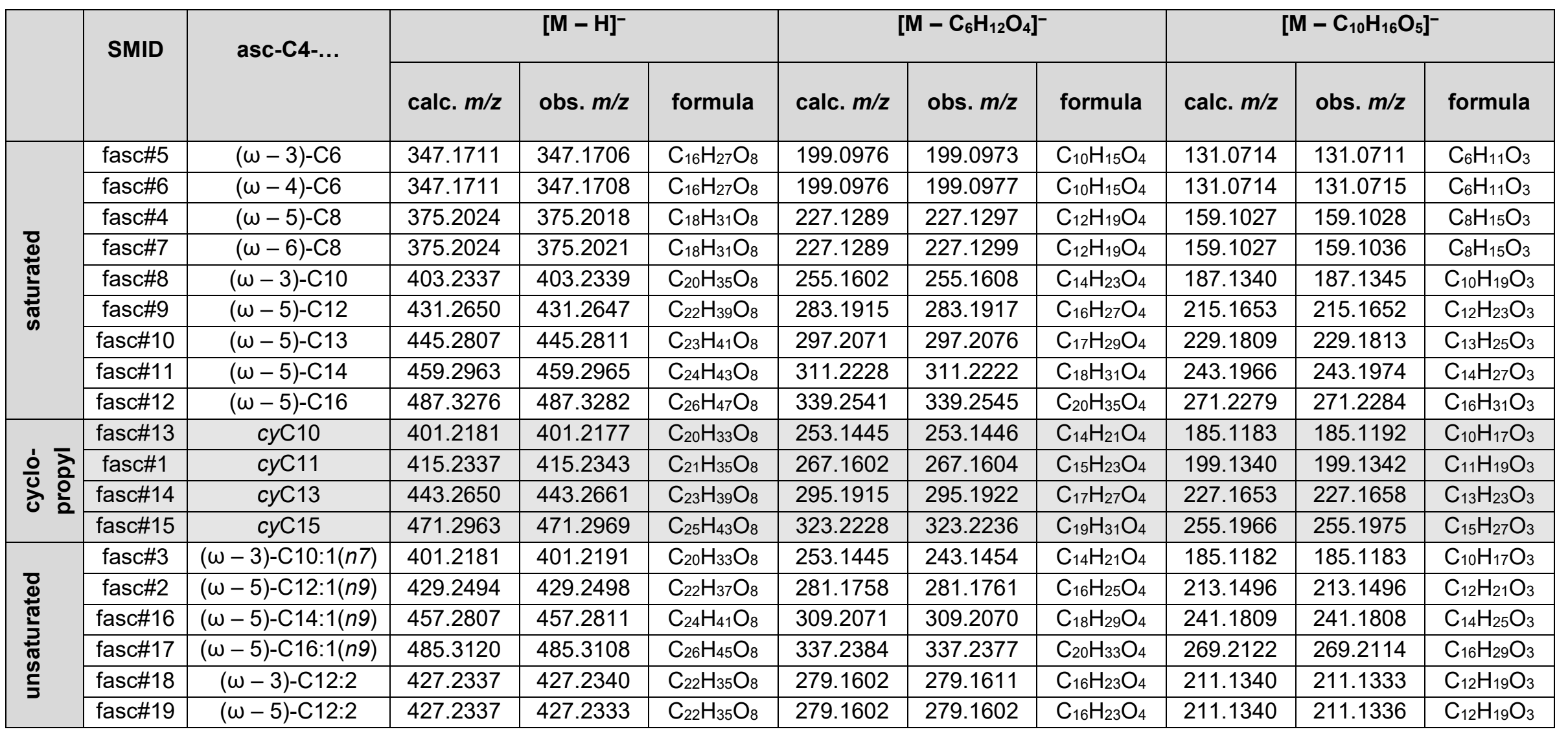




\section{Supporting Figures}

Figure S1: enantioselective gas chromatography using heptakis(2,3-O-dimethyl-6-O-tertbutyldimethylsilyl)- $\beta$-cyclodextrin as chiral phase: a) separation of racemic $( \pm)-5-0$-tertbutyldimethylsilyloxy-cis-2,3-methylenepentan-1-ol (11); b) analysis of (-)-(2S,3S)-5-O-tertbutyldimethylsilyloxy-cis-2,3-methylenepentan-1-ol (11) with ee $=94 \%$; c) co-injection of $a+b$ $(1: 1)$.
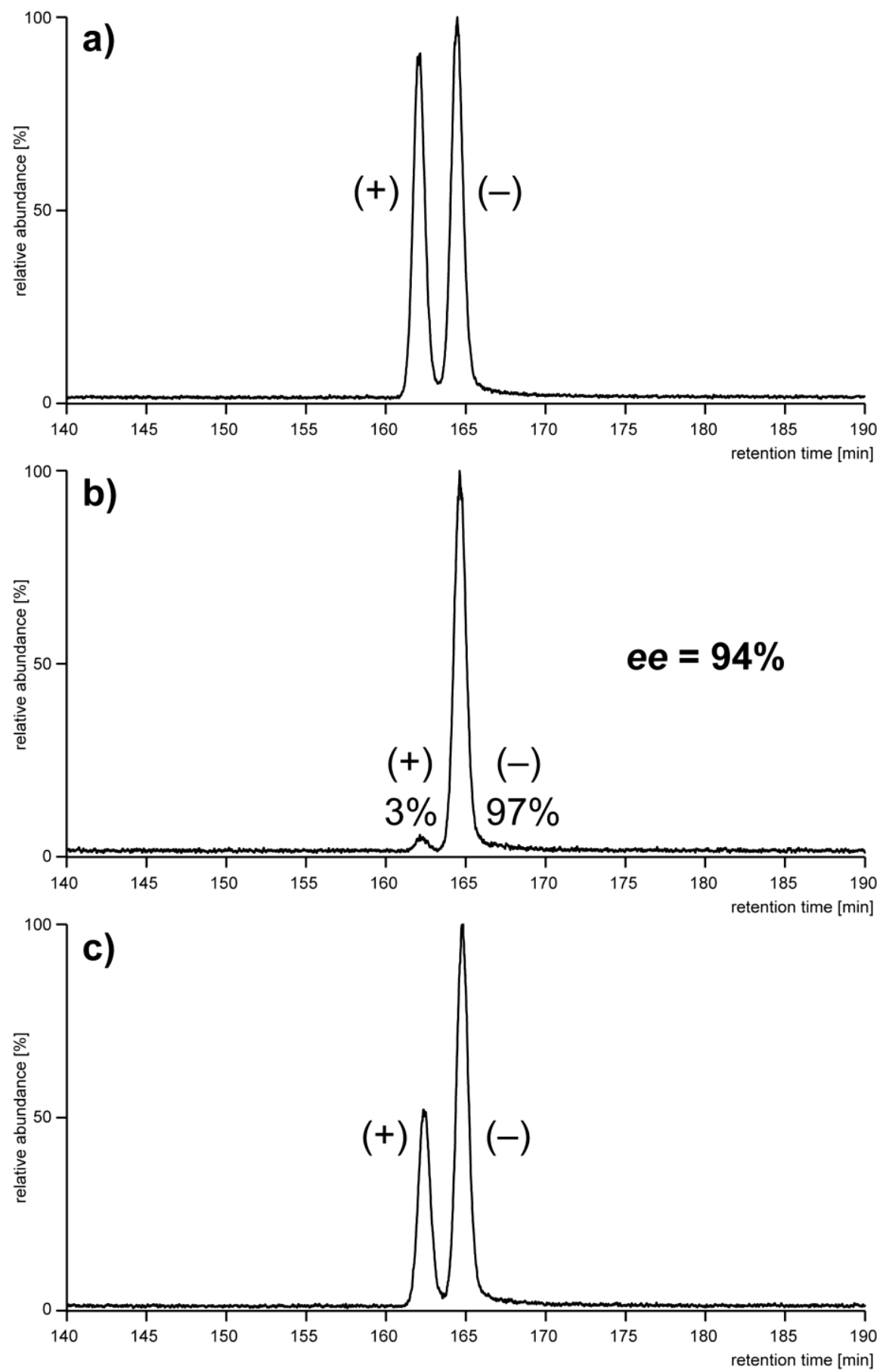
Figure S2: enantioselective gas chromatography using heptakis(2,3,6-tri-O-methyl)- $\beta$ cyclodextrin as chiral phase: a) separation of racemic $( \pm)-1-$ hexen-3-yl acetate $(( \pm)-15)$; b) analysis of $(-)-(3 S)-1$-hexen-3-yl acetate $((-)-15)$ with ee $=98 \%$; $)$ co-injection of $a+b(1: 10)$.
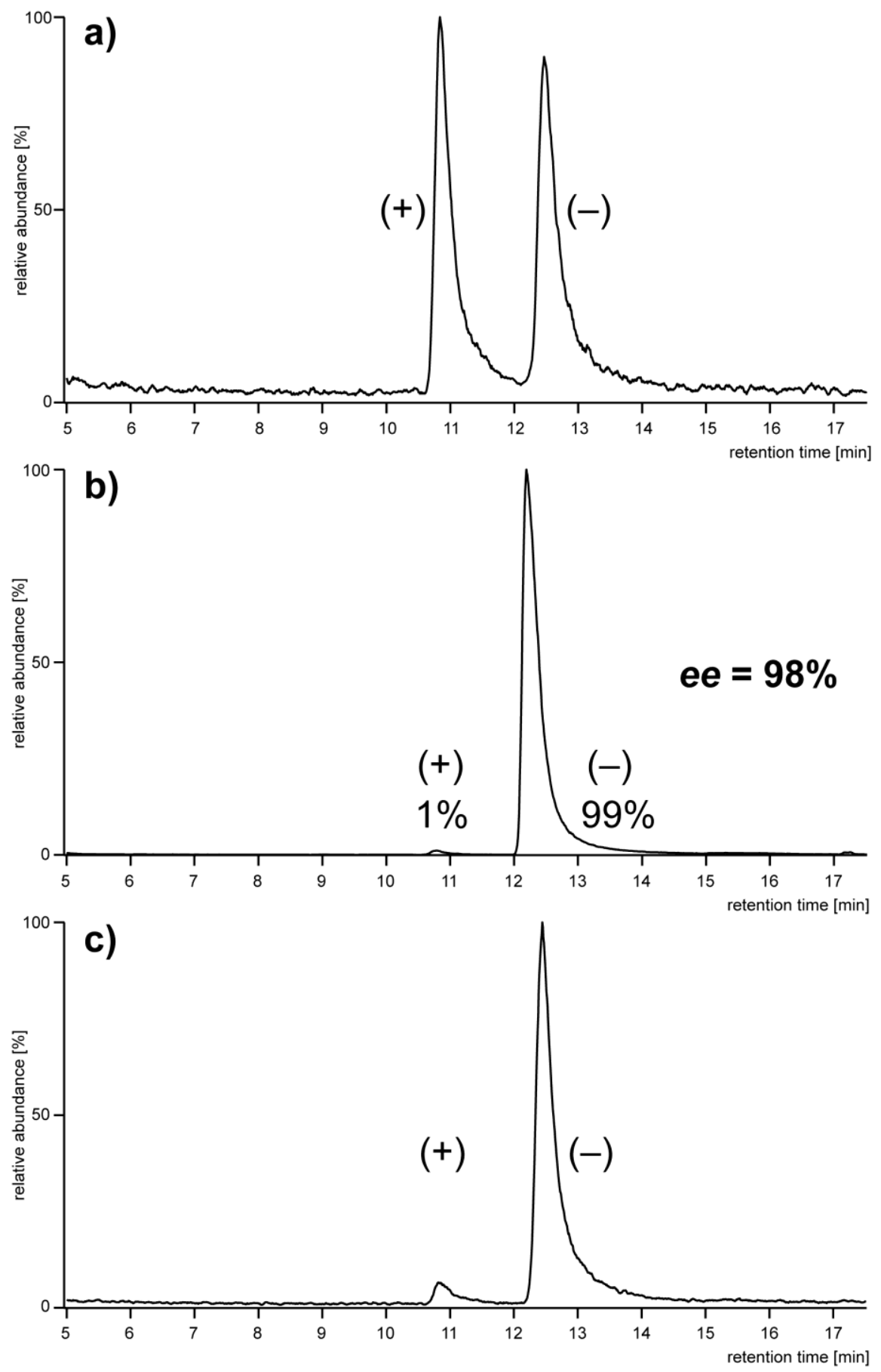


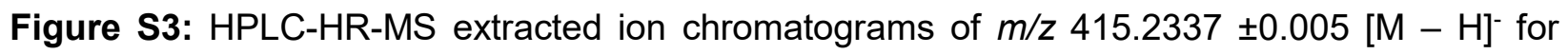
natural asc-C4-cyC11 (fasc\#1, 3) from C. remanei PB4641 (A) and the synthetic product (B).
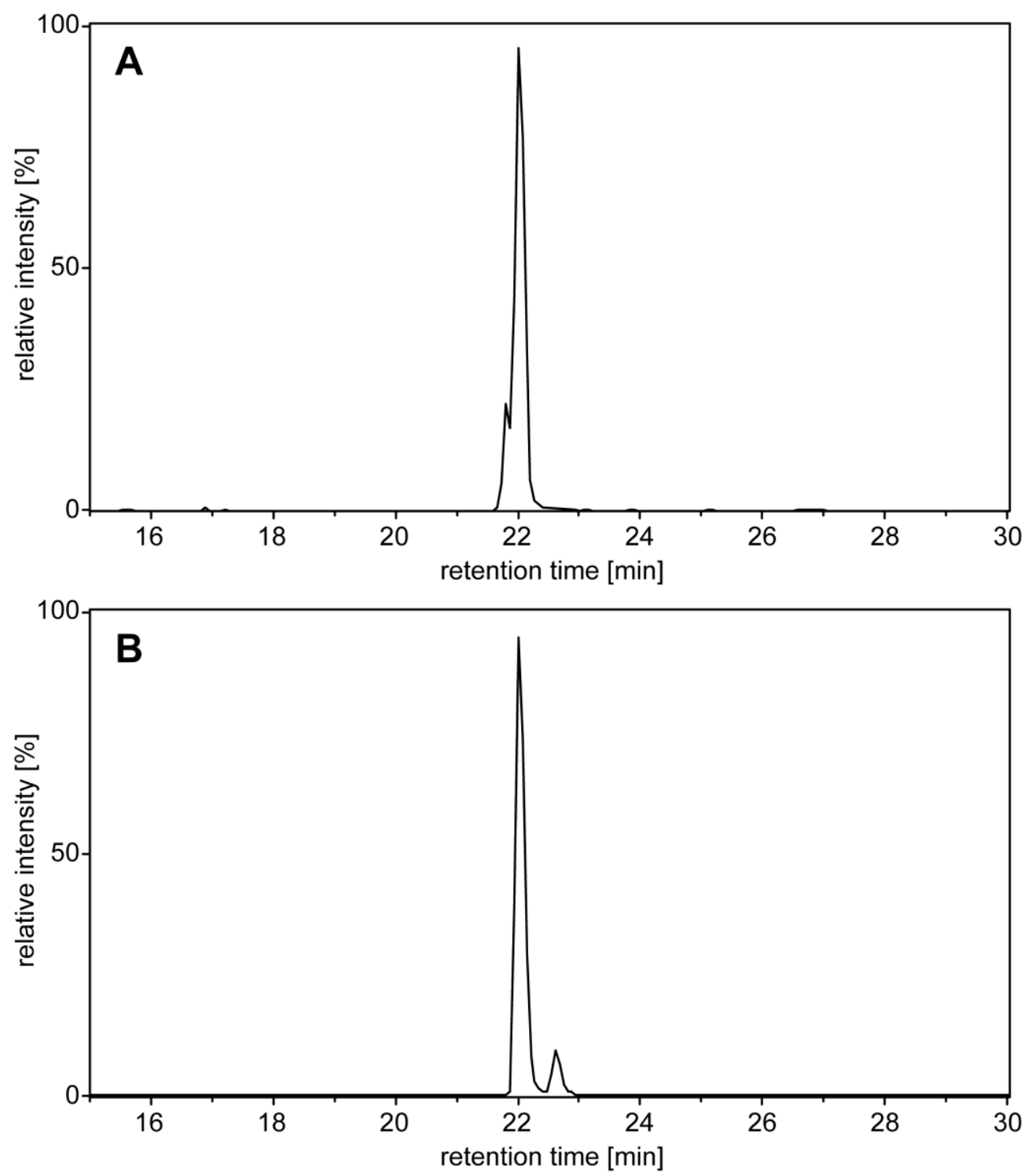
Figure S4a: HPLC-ESI-(-)-HR-MS spectra of natural asc-C4-cyC11 (fasc\#1, 3) isolated from C. remanei PB4641 (A, C) and the synthetic product (B, D).
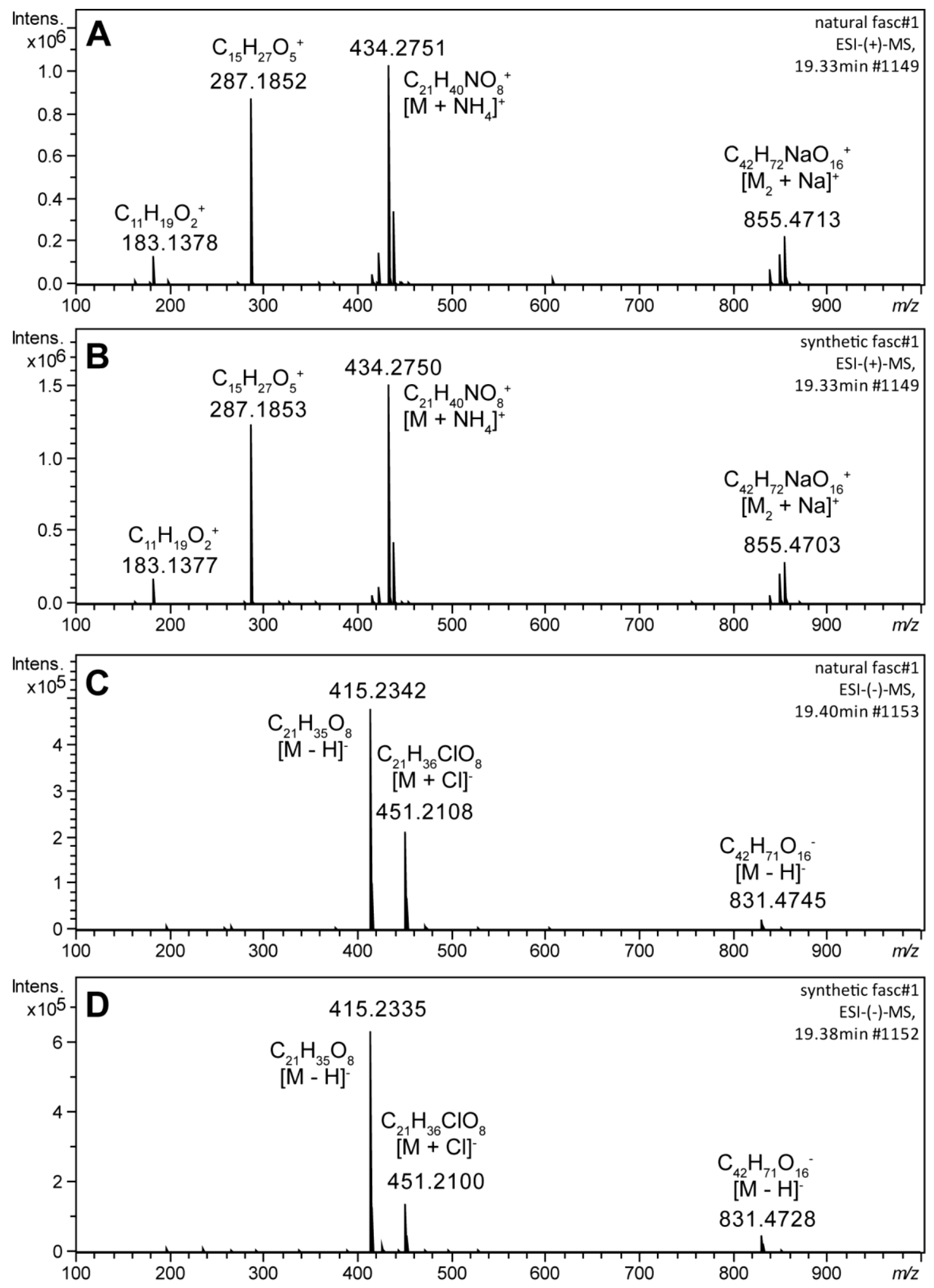
Figure S4b: HPLC-ESI-(+)-HR-MS/MS spectra of natural asc-C4-cyC11 (fasc\#1, 3) isolated from C. remanei PB4641 (A, C) and the synthetic product (B, D).
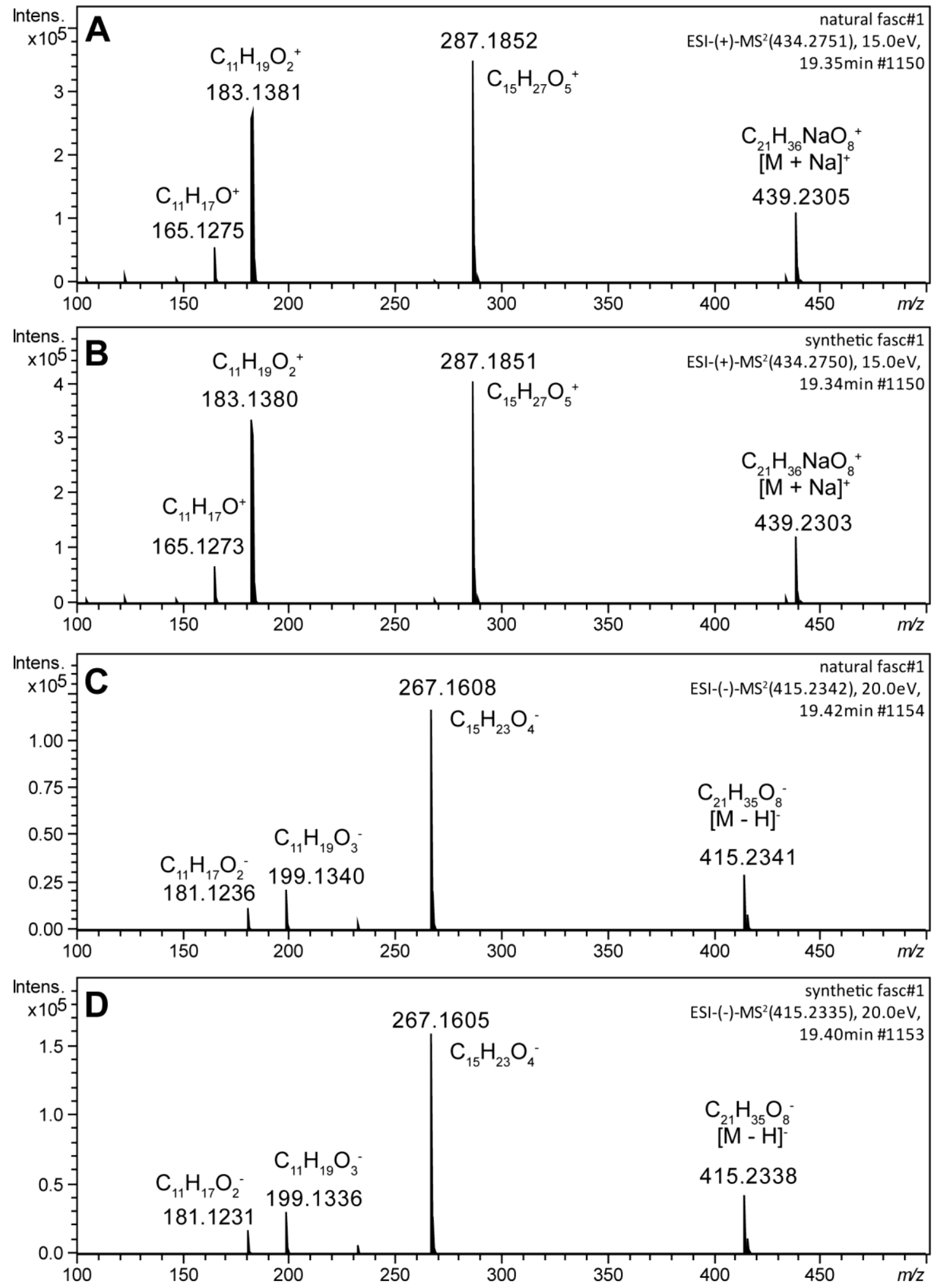
Figure S5a: Upfield sections of $400 \mathrm{MHz}{ }^{1} \mathrm{H}$ NMR spectra of $(\mathbf{A})$ natural asc-C4-cyC11 (fasc\#1, 3) isolated from $C$. remanei PB4641 and (B) the synthetic product in $\mathrm{CD}_{3} \mathrm{OD}$. Chemical shifts of the $\alpha$-methylene protons of the fatty acid moiety are highly variable as shown in Figure S5c.

A

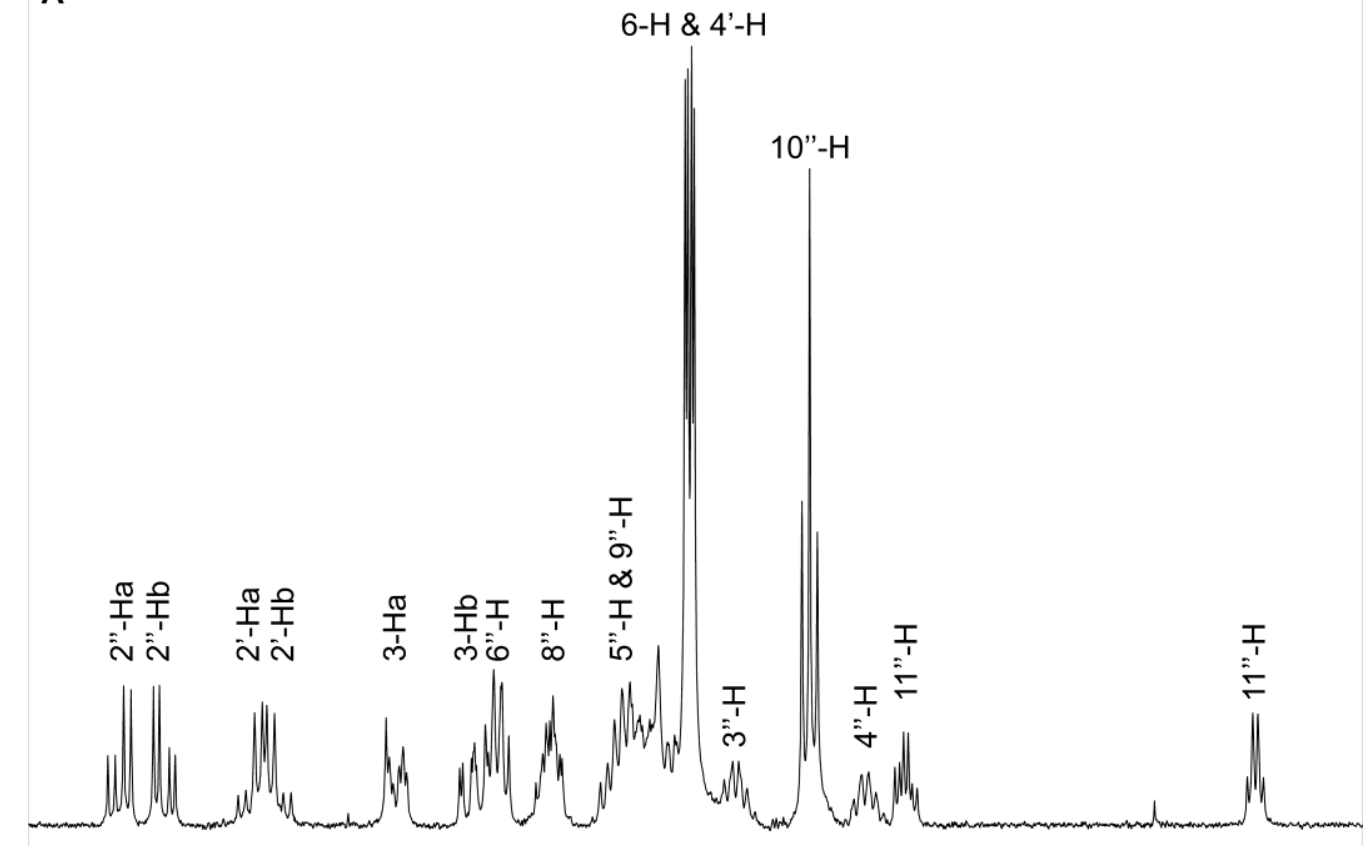

$\begin{array}{lllllllllllllllllllllllllllllllllllllllllllll}2.7 & 2.6 & 2.5 & 2.4 & 2.3 & 2.2 & 2.1 & 2.0 & 1.9 & 1.8 & 1.7 & 1.6 & 1.5 & 1.4 & 1.3 & 1.2 & 1.1 & 1.0 & 0.9 & 0.8 & 0.7 & 0.6 & 0.5 & 0.4 & 0.3 & 0.2 & 0.1 & 0.0 & -0.1 & -0.2 & -0.3\end{array}$

B

6-H \& 4'-H

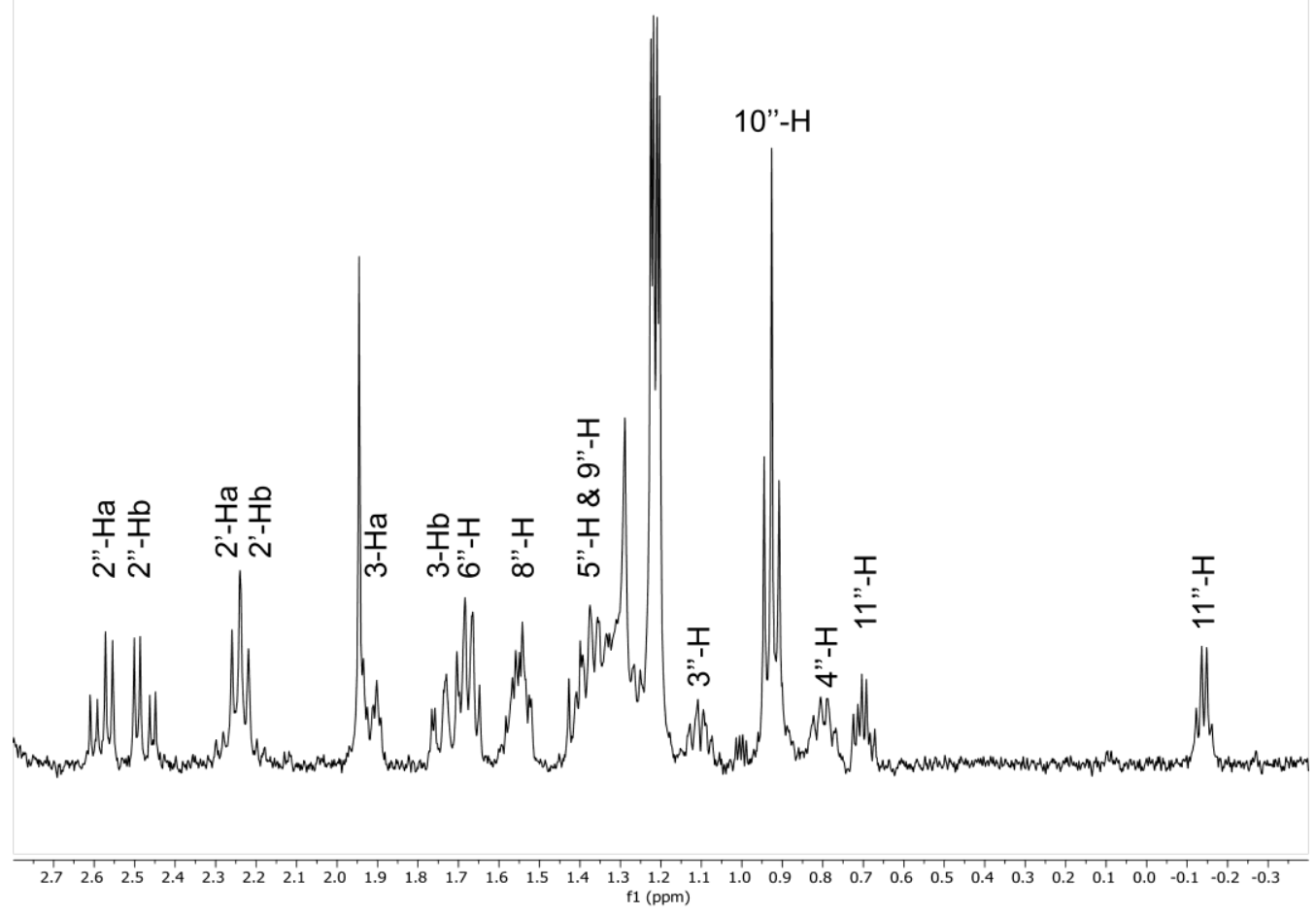


Figure S5b: Downfield sections of $400 \mathrm{MHz}{ }^{1} \mathrm{H}$ NMR spectra of (A) natural asc-C4-cyC11 $\left(\right.$ fasc\#1, 3) isolated from $C$. remanei PB4641 and $(B)$ the synthetic product in $\mathrm{CD}_{3} \mathrm{OD}$.

A

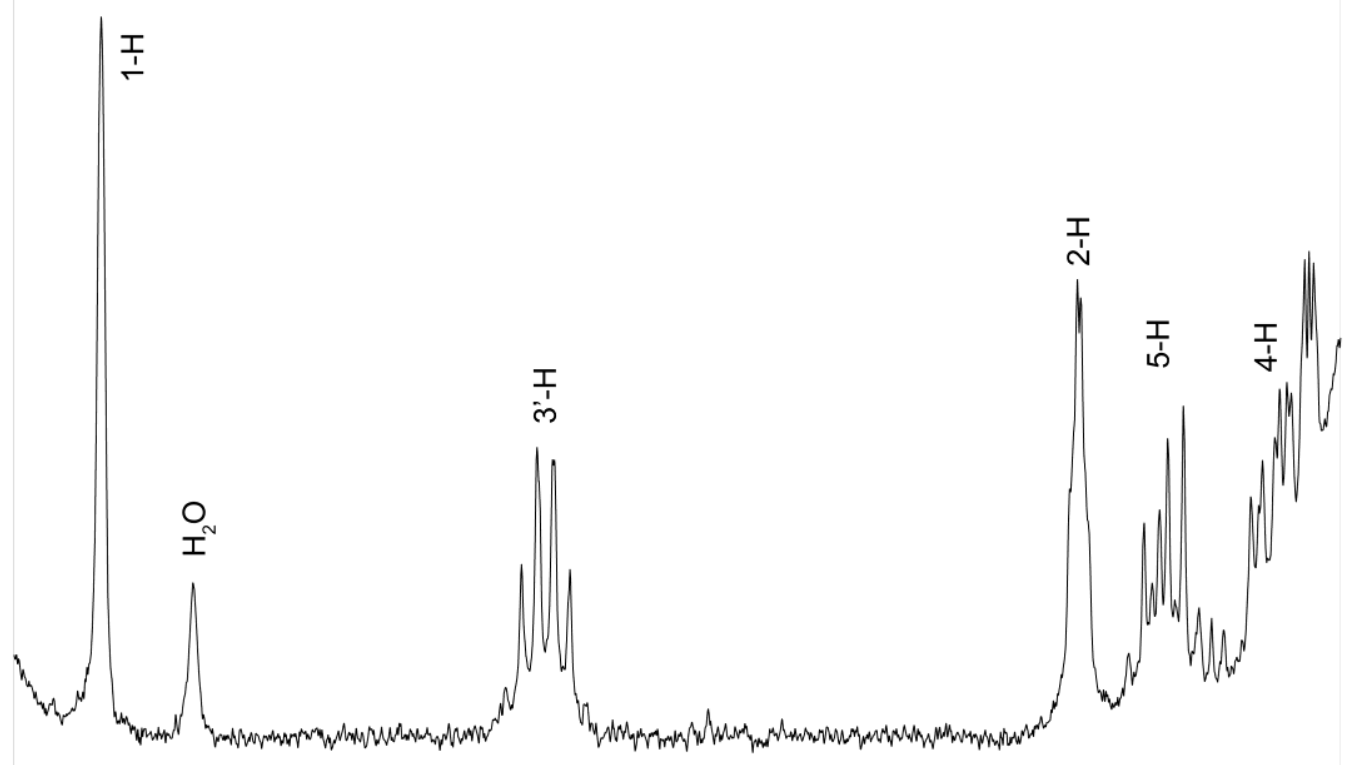

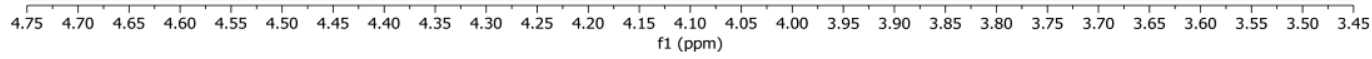

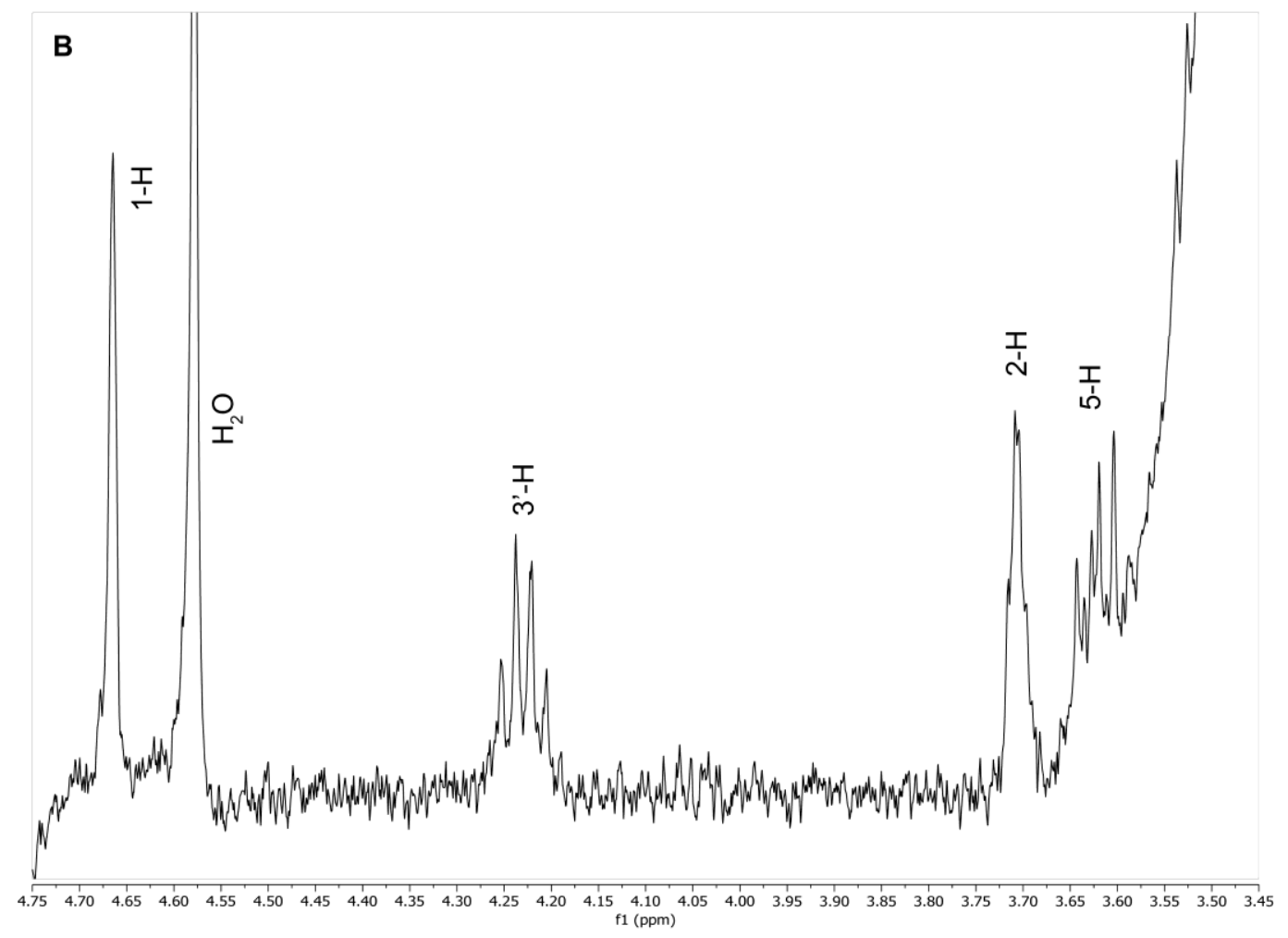


Figure S5c: Comparative analysis of ${ }^{1} \mathrm{H}$ NMR spectra $\left(400 \mathrm{MHz}, \mathrm{CD}_{3} \mathrm{OD}\right.$, sections from $2.00-$ $2.75 \mathrm{ppm}$ ) showing both $\alpha-$ methylene groups of (A) synthetic asc-C4-cyC11 (fasc\#1, 3), (B-E) natural asc-C4-cyC11 (fasc\#1, 3) isolated from C. remanei PB4641 by solid phase extraction on RP-C18 and preparative HPLC, and (F) the isolated natural asc-C4-cyC11 (fasc\#1, 3) after addition of equimolar amounts of sodium deuteroxide, reveals a strong $\mathrm{pH}$ dependence of the chemical shifts of the a-methylene protons of the cis-3,4-methylenedecanoic acid moiety (2'-Ha and $\left.2^{\prime}-\mathrm{Hb}\right)$.
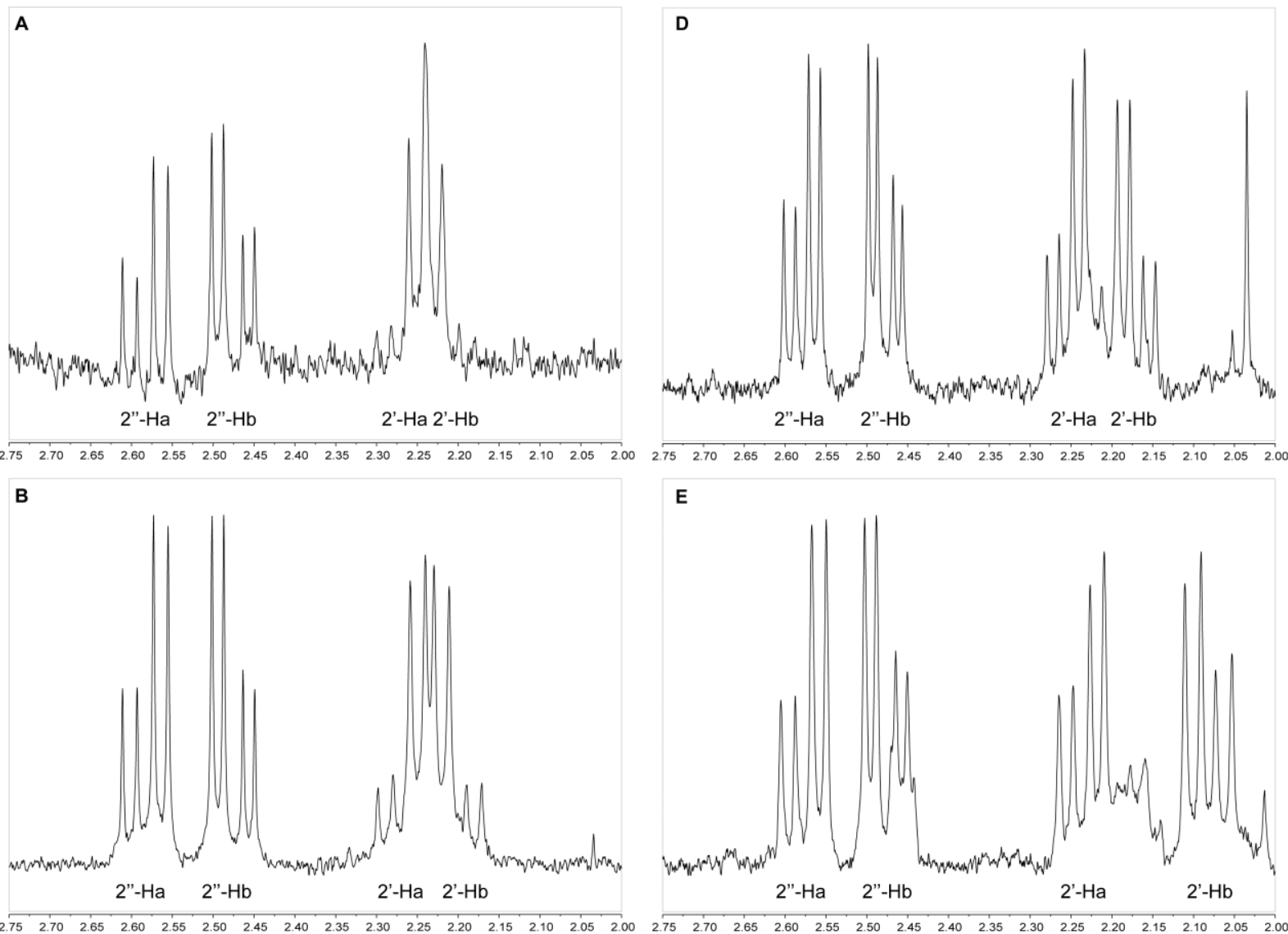

E

C
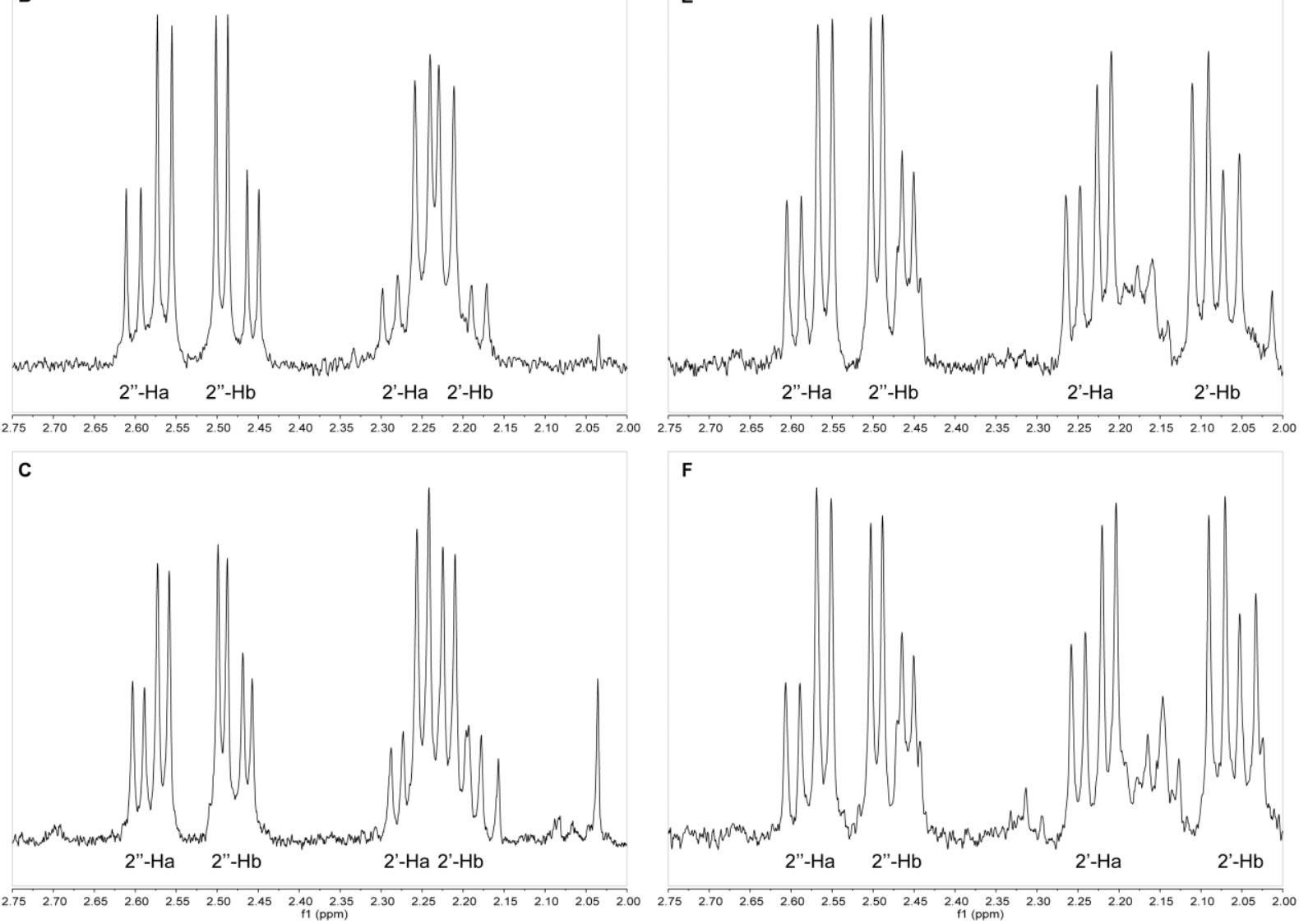
Figure S6a: Structures of fatty acid ascarosides carrying saturated fatty acid building blocks identified in the exometabolomes of $C$. remanei and $C$. latens.<smiles>CCCCC[C@H](CC(=O)O)OC(=O)C[C@@H](C)O[C@H]1O[C@H](C)[C@@H](O)C[C@H]1O</smiles><smiles>CCCC(CC(=O)O)OC(=O)C[C@@H](C)O[C@H]1O[C@H](C)[C@@H](O)C[C@H]1O</smiles><smiles>CCCC[C@H](OC(=O)C[C@H](C)O[C@@H]1O[C@H](C)[C@@H](O)C[C@H]1O)C(=O)O</smiles><smiles>CCCCCC[C@H](OC(=O)C[C@H](C)O[C@@H]1O[C@H](C)[C@@H](O)C[C@H]1O)C(=O)O</smiles><smiles>CCCC(C)CCCCCC(=O)OC(=O)C[C@@H](C)O[C@@H]1O[C@H](C)[C@@H](O)C[C@H]1O</smiles><smiles>CCCCCC(CCCCCC(=O)O)OC(=O)C[C@@H](C)O[C@@H]1O[C@H](C)[C@@H](O)C[C@H]1O</smiles>

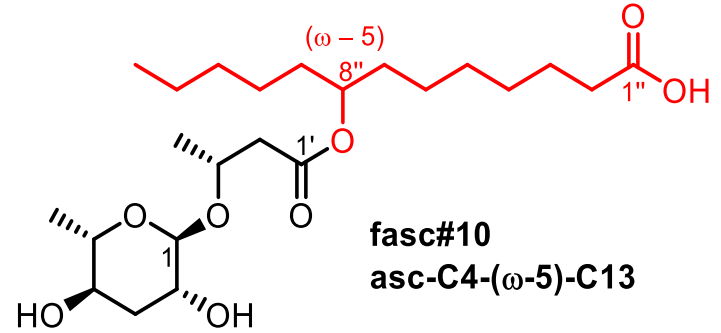<smiles>CCCCCC(CCCCCCCC(=O)O)OC(=O)C[C@@H](C)O[C@@H]1O[C@H](C)[C@@H](O)C[C@H]1O</smiles><smiles>CCCCC[C@@H](CCCCCCCCCC(=O)O)OC(=O)C[C@@H](C)O[C@@H]1O[C@H](C)[C@@H](O)C[C@H]1O</smiles> 
Figure S6b: Structures of fatty acid ascarosides carrying cyclopropyl fatty acid building blocks identified in the exometabolomes of $C$. remanei and $C$. latens.
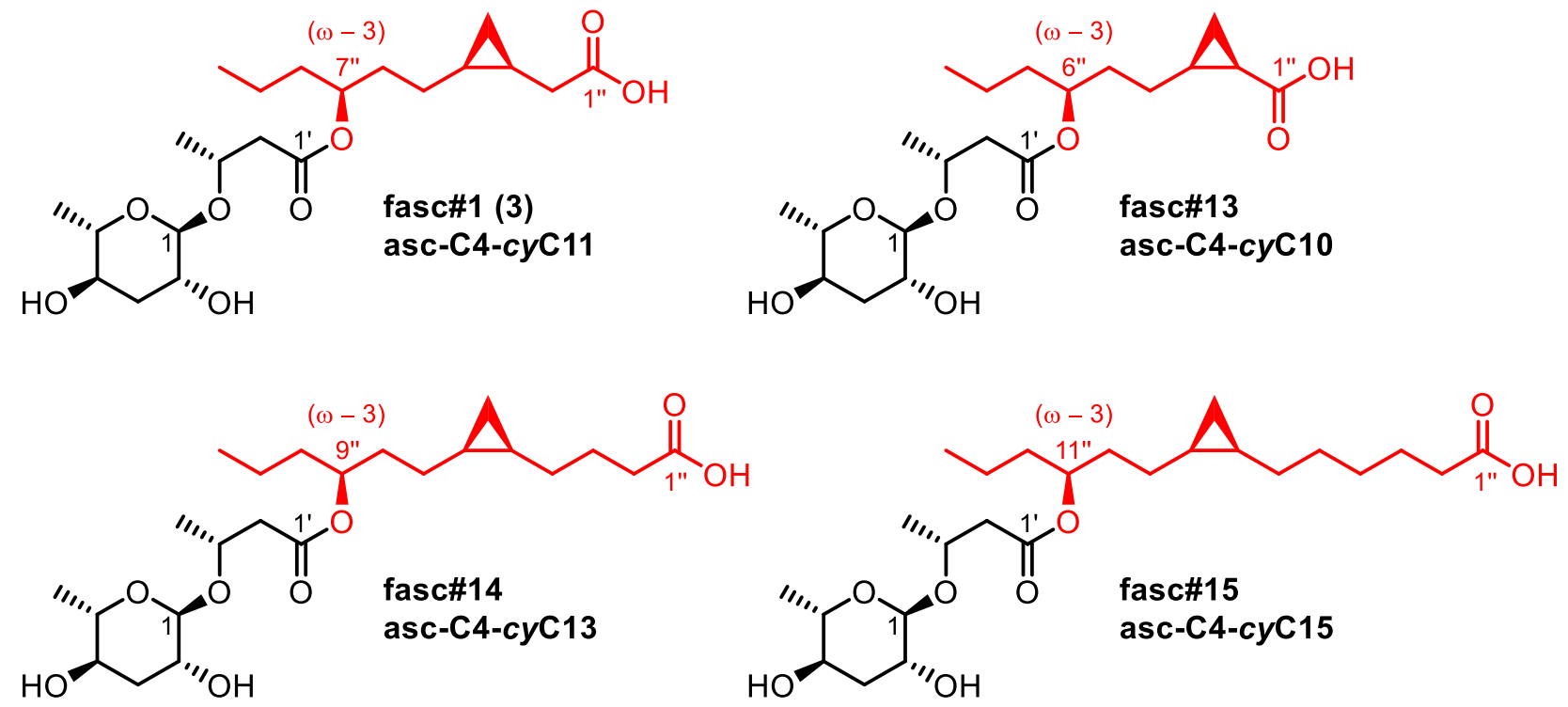
Figure S6c: Structures of fatty acid ascarosides carrying unsaturated fatty acid building blocks identified in the exometabolomes of $C$. remanei and $C$. latens.<smiles>CCC[C@H](CC/C=C\CC(=O)O)OC(=O)C[C@H](C)O[C@@H]1O[C@H](C)[C@@H](O)C[C@H]1O</smiles><smiles>CCCCC[C@H](CC/C=C\CC(=O)O)OC(=O)C[C@H](C)O[C@@H]1O[C@H](C)[C@@H](O)C[C@H]1O</smiles><smiles>CCCCC[C@H](CC/C=C/CCCC(=O)O)OC(=O)C[C@@H](C)O[C@@H]1O[C@H](C)[C@@H](O)C[C@H]1O</smiles>

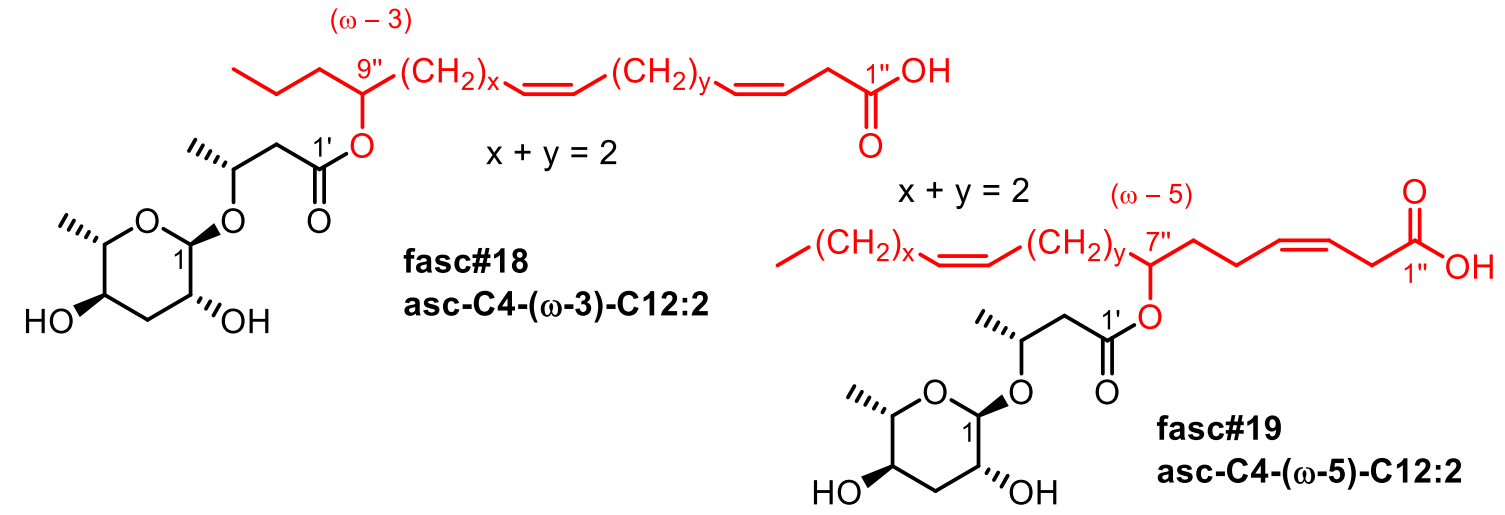


Figure S7a: Comparative HPLC-ESI-(-)-MS ${ }^{3}$ analysis of a C. remanei exometabolome fraction enriched with fatty acid ascarosides and its $\mathrm{Pd} / \mathrm{C}$-catalyzed hydrogenation products indicates an inert cyclopropyl fatty acid moiety for asc-C4-cyC11 (fasc\#1, 3).

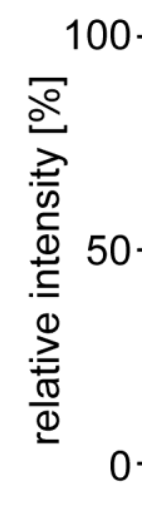
C. remanei
23.15

fasc mixture

asc-C4-cyc11

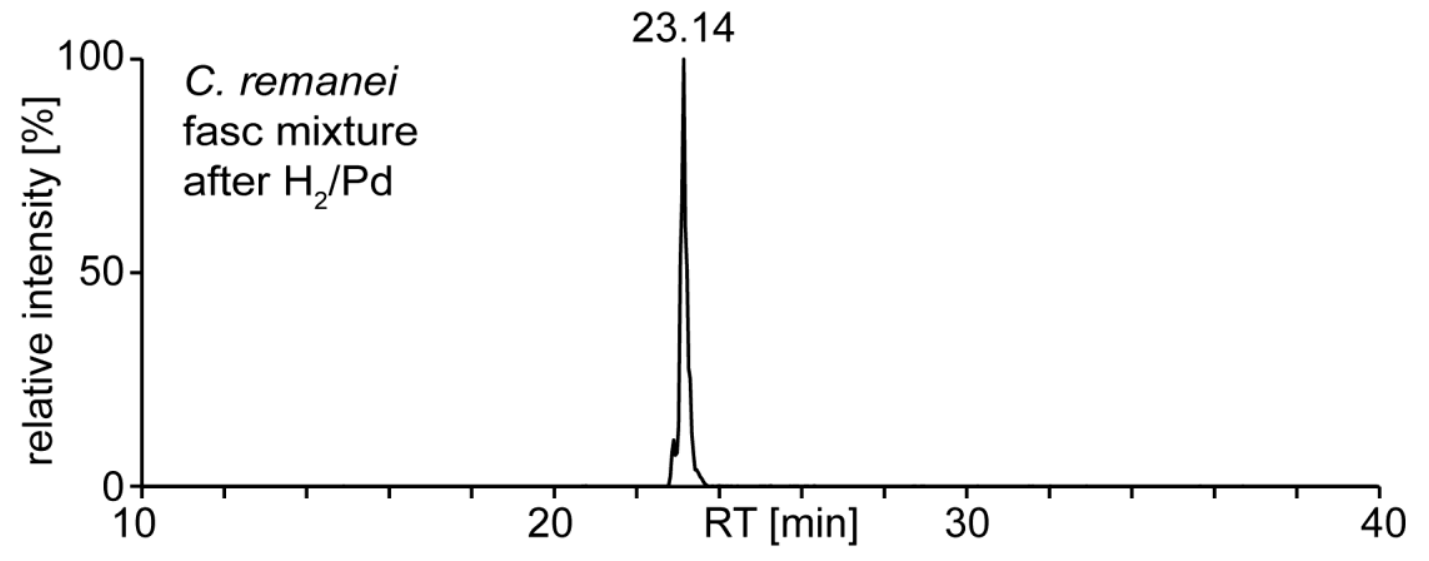


Figure S7b: Comparative HPLC-ESI-(-)-MS ${ }^{3}$ analysis of a C. remanei exometabolome fraction enriched with fatty acid ascarosides and its $\mathrm{Pd} / \mathrm{C}$-catalyzed hydrogenation products indicates an inert cyclopropyl fatty acid moiety for asc-C4-cyC13 (fasc\#14).
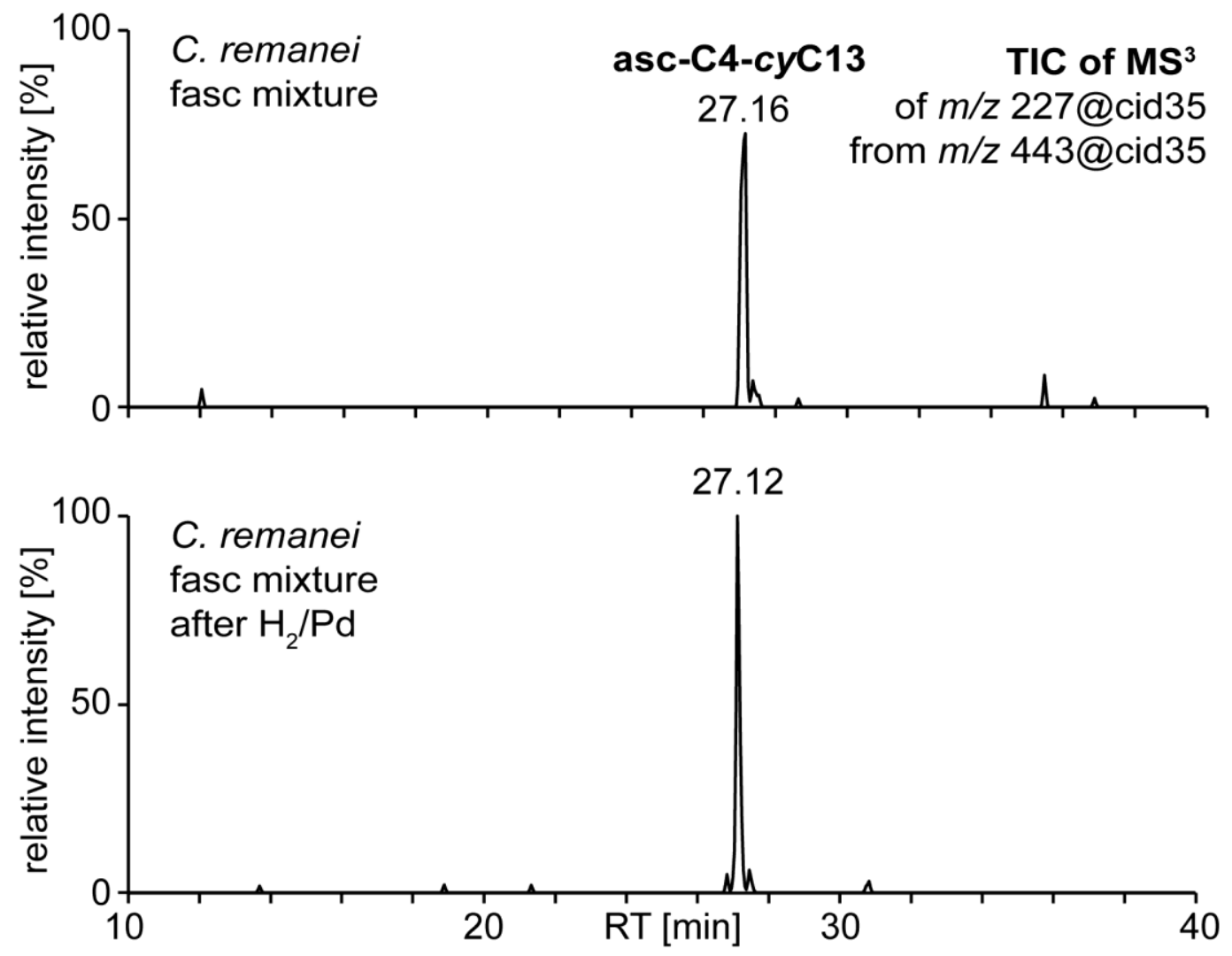
Figure S7c: Comparative HPLC-ESI-(-)-MS ${ }^{3}$ analysis of a C. remanei exometabolome fraction enriched with fatty acid ascarosides and its $\mathrm{Pd} / \mathrm{C}$-catalyzed hydrogenation products indicates a monounsaturated decenoate moiety for asc-C4-C10:1 (fasc\#3, 5) along with an inert cyclopropyl fatty acid moiety for the coeluting asc-C4-cyC10 (fasc\#13).

asc-C4-C10:1
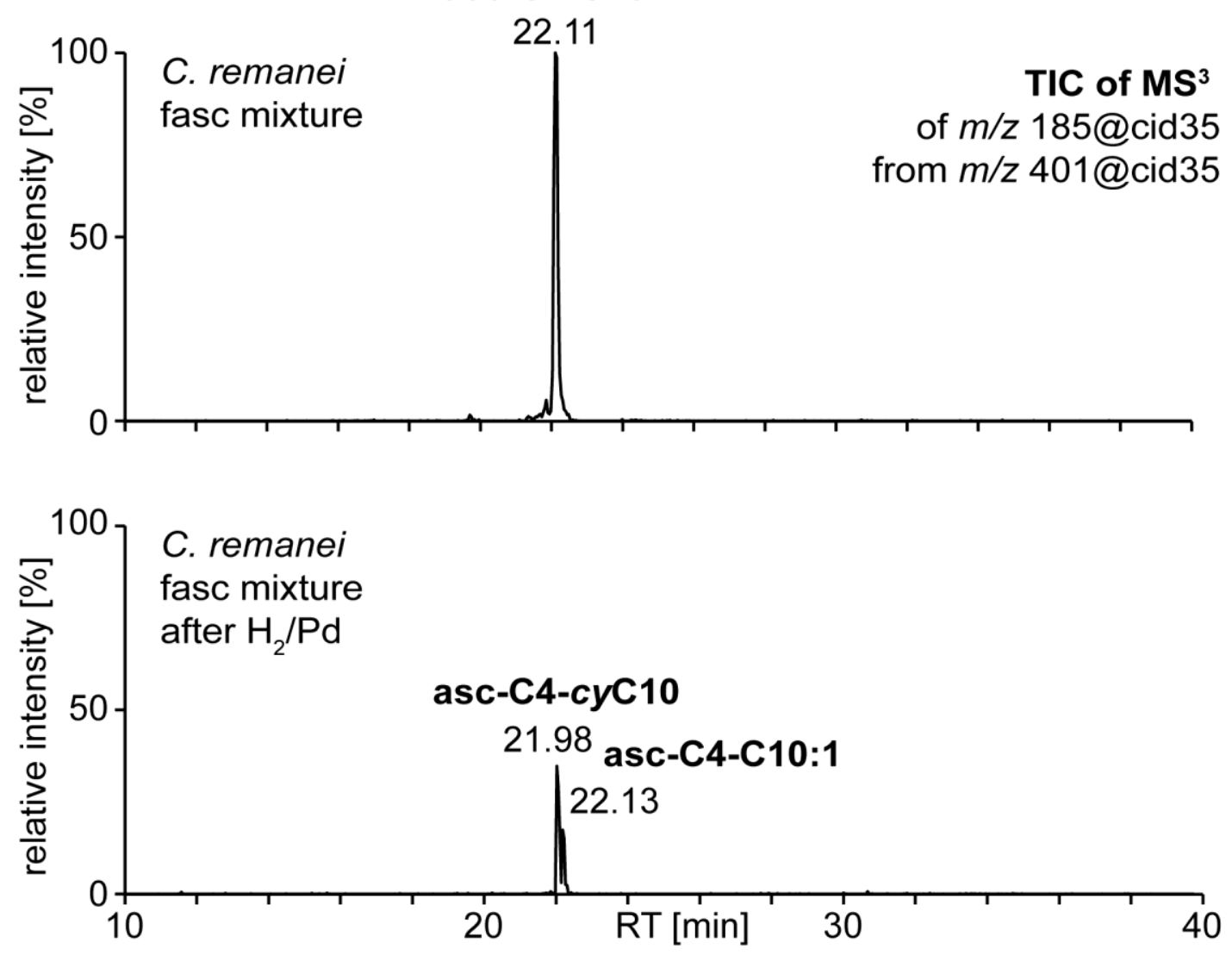
Figure S7d: Comparative HPLC-ESI-(-)-MS ${ }^{3}$ analysis of a C. remanei exometabolome fraction enriched with fatty acid ascarosides and its $\mathrm{Pd} / \mathrm{C}$-catalyzed hydrogenation products indicates a monounsaturated dodecenoate moiety for asc-C4-C12:1 (fasc\#2, 4).
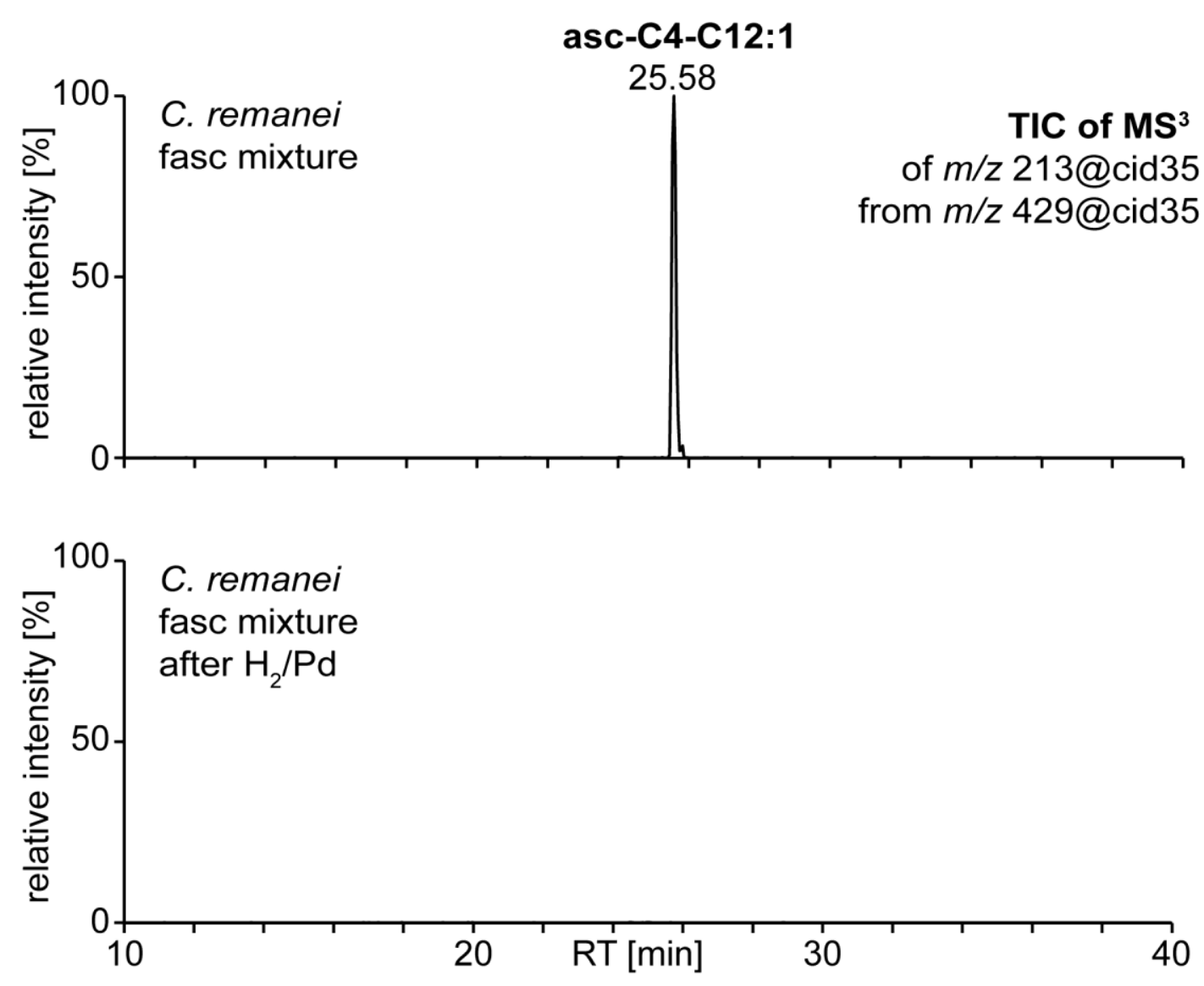
Figure S7e: Comparative HPLC-ESI-(-)-MS ${ }^{3}$ analysis of a C. remanei exometabolome fraction enriched with fatty acid ascarosides and its $\mathrm{Pd} / \mathrm{C}$-catalyzed hydrogenation products indicates a monounsaturated tetradecenoate moiety for asc-C4-C14:1 (fasc\#16).
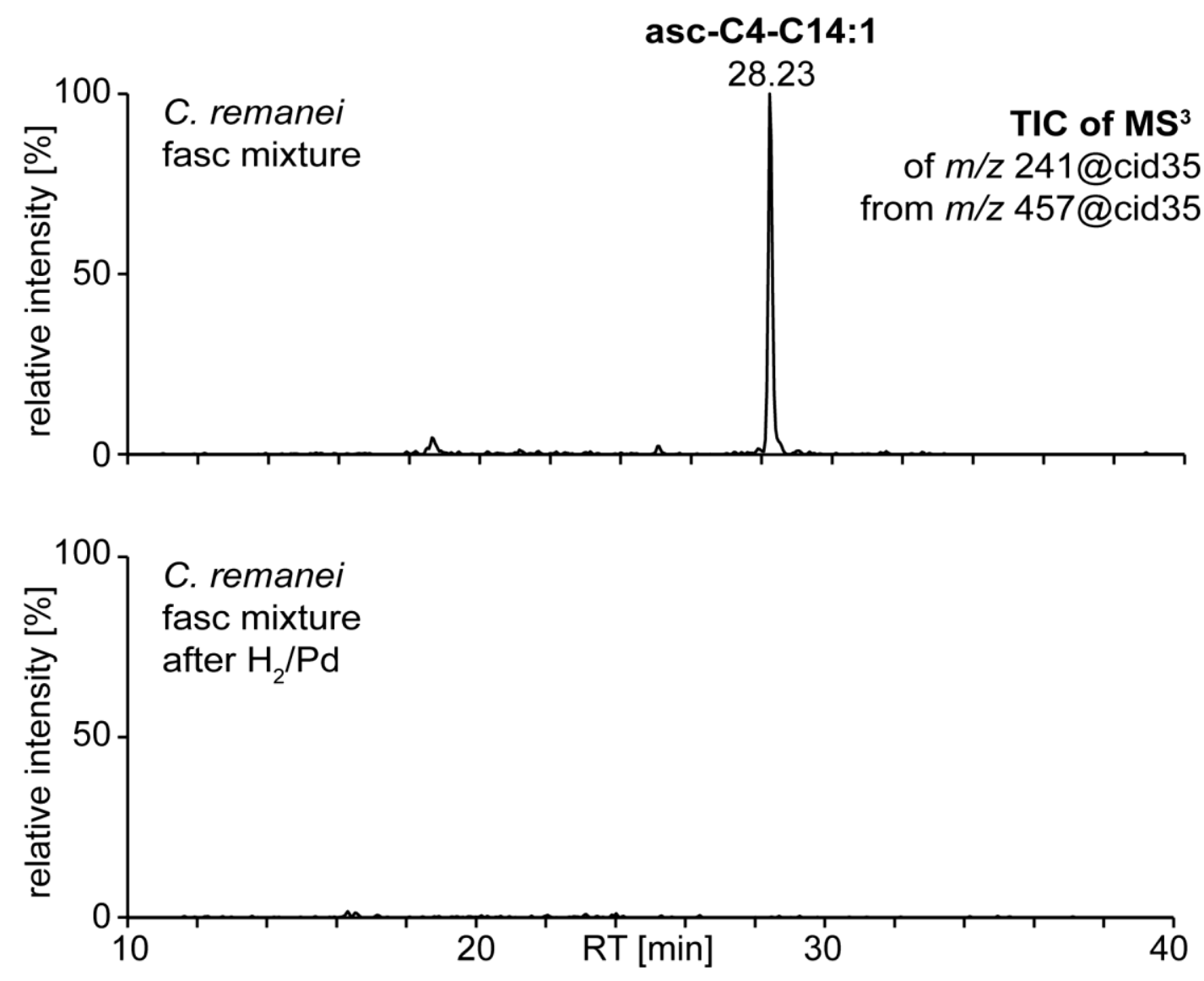
Figure S7f: Comparative HPLC-ESI-(-)-MS ${ }^{3}$ analysis of a C. remanei exometabolome fraction enriched with fatty acid ascarosides and its $\mathrm{Pd} / \mathrm{C}$-catalyzed hydrogenation products indicates a monounsaturated hexadecenoate moiety for asc-C4-C16:1 (fasc\#17).
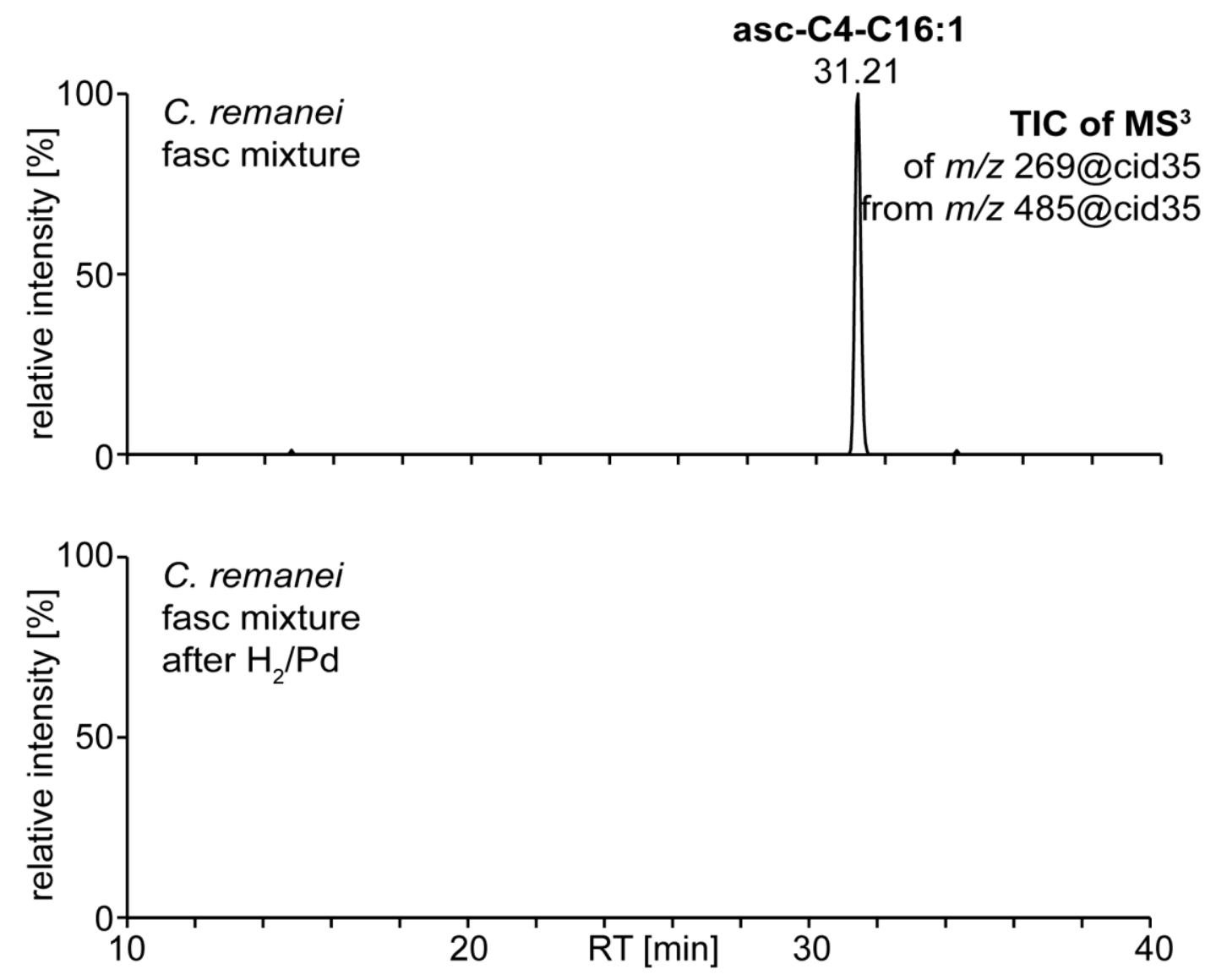
Figure S7g: Comparative HPLC-ESI-(-)-MS ${ }^{3}$ analysis of a C. remanei exometabolome fraction enriched with fatty acid ascarosides and its $\mathrm{Pd} / \mathrm{C}$-catalyzed hydrogenation products indicates unsaturated dodecadienoate moieties for asc-C4-( $\omega-3)-C 12: 2$ (fasc\#18) and for asc-C4-( $\omega-5)$ C12:2 (fasc\#19).
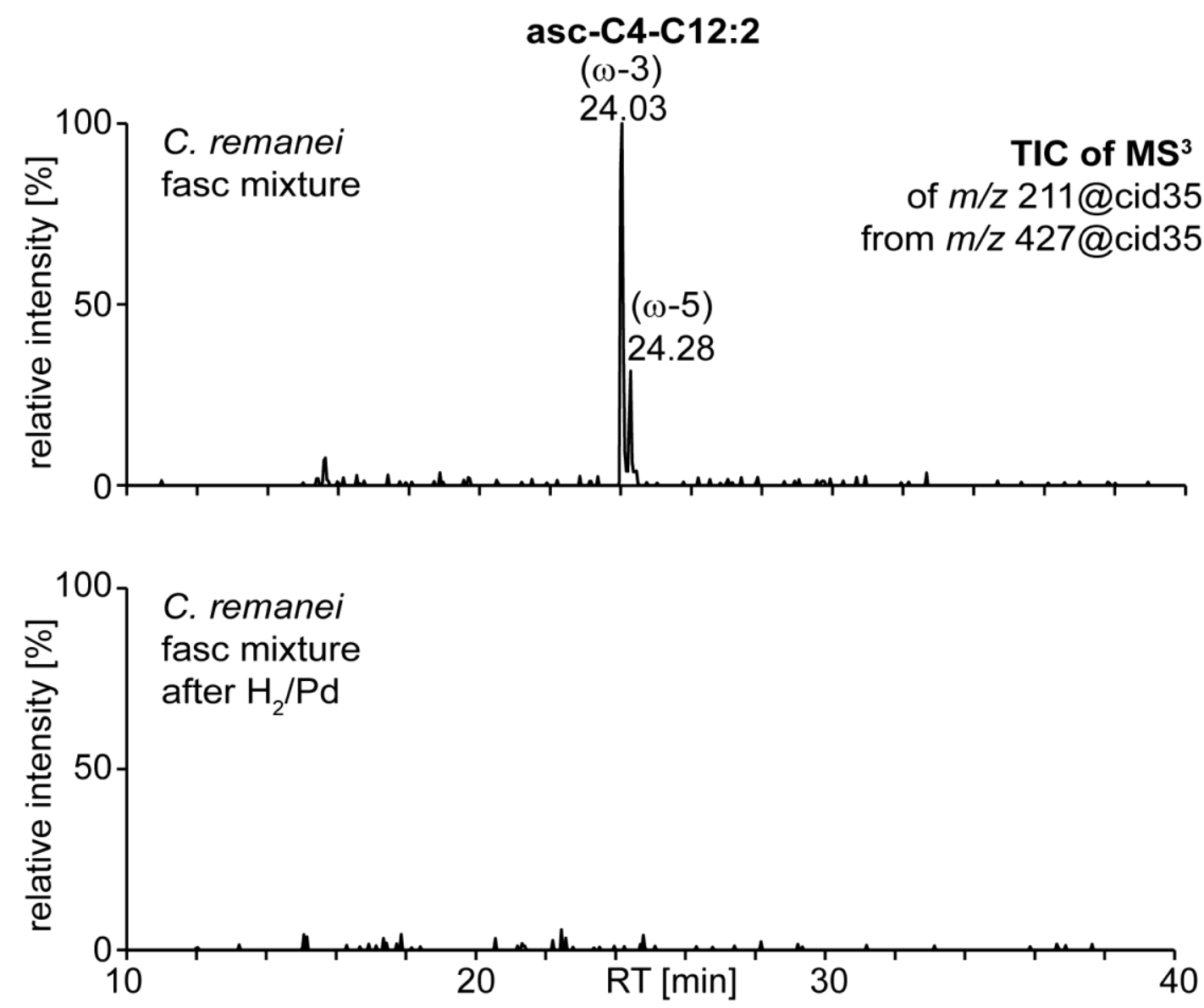
Figure S8a: Comparative analysis of ESI-(-)-MS² spectra of semisynthetic asc-C4-C12 (fasc\#9) and $\left[3,4-D_{2}\right]$-asc-C4-C12 ([D $]$-fasc\#9) prepared by $P d / C$-catalyzed hydrogenation and deuterogenation of asc-C4-C12:1 (fasc\#2, 4) from C. remanei, respectively.

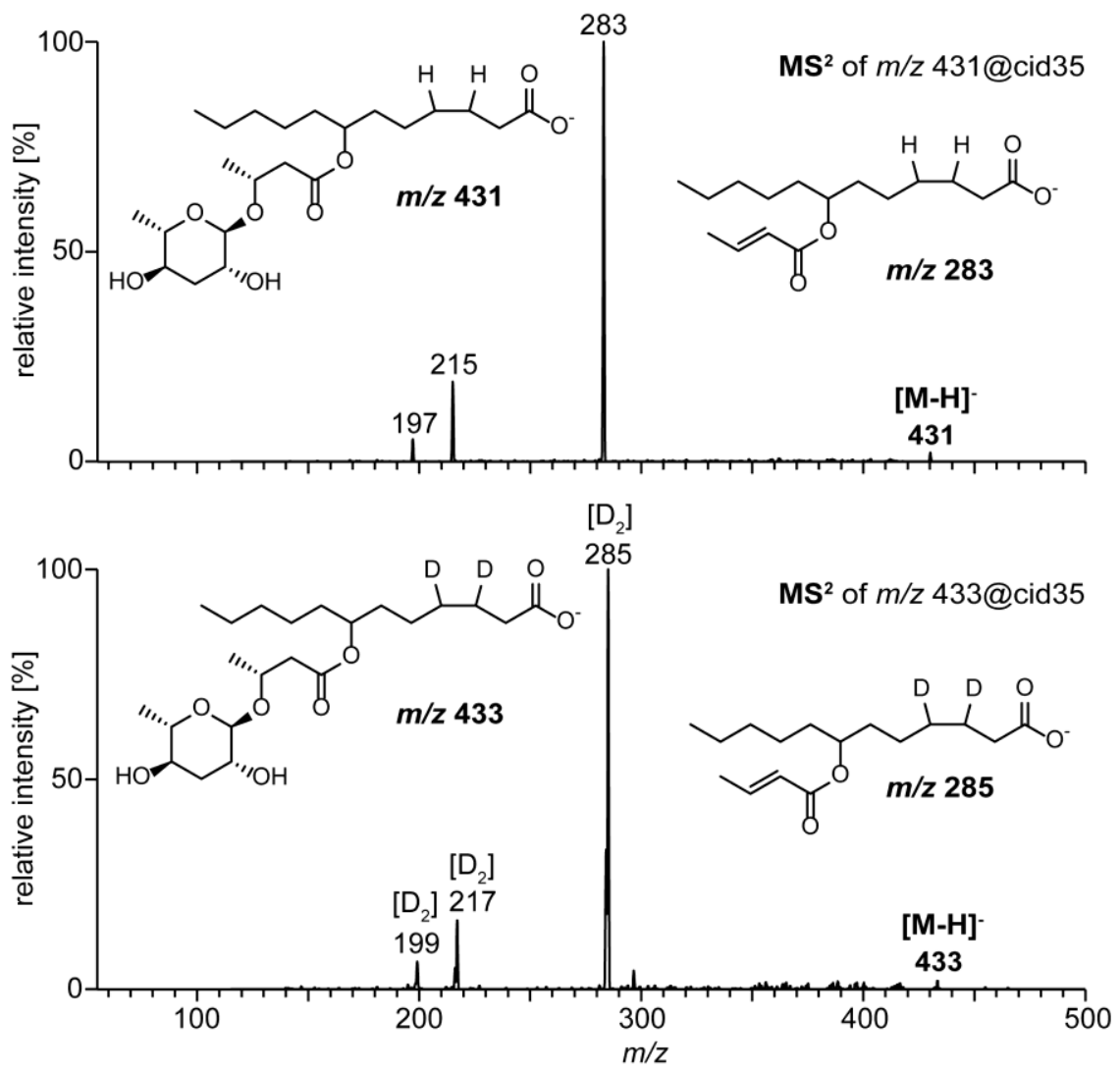


Figure S8b: Comparative analysis of ESI-(-)-MS ${ }^{3}$ spectra of semisynthetic asc-C4-C12 (fasc\#9) and [3,4-D $\left.\mathrm{D}_{2}\right]$-asc-C4-C12 $\left(\left(\left[\mathrm{D}_{2}\right]\right.\right.$-fasc\#9) prepared by $\mathrm{Pd} / \mathrm{C}$-catalyzed hydrogenation and deuterogenation of asc-C4-C12:1 (fasc\#2, 4) from C. remanei , respectively.

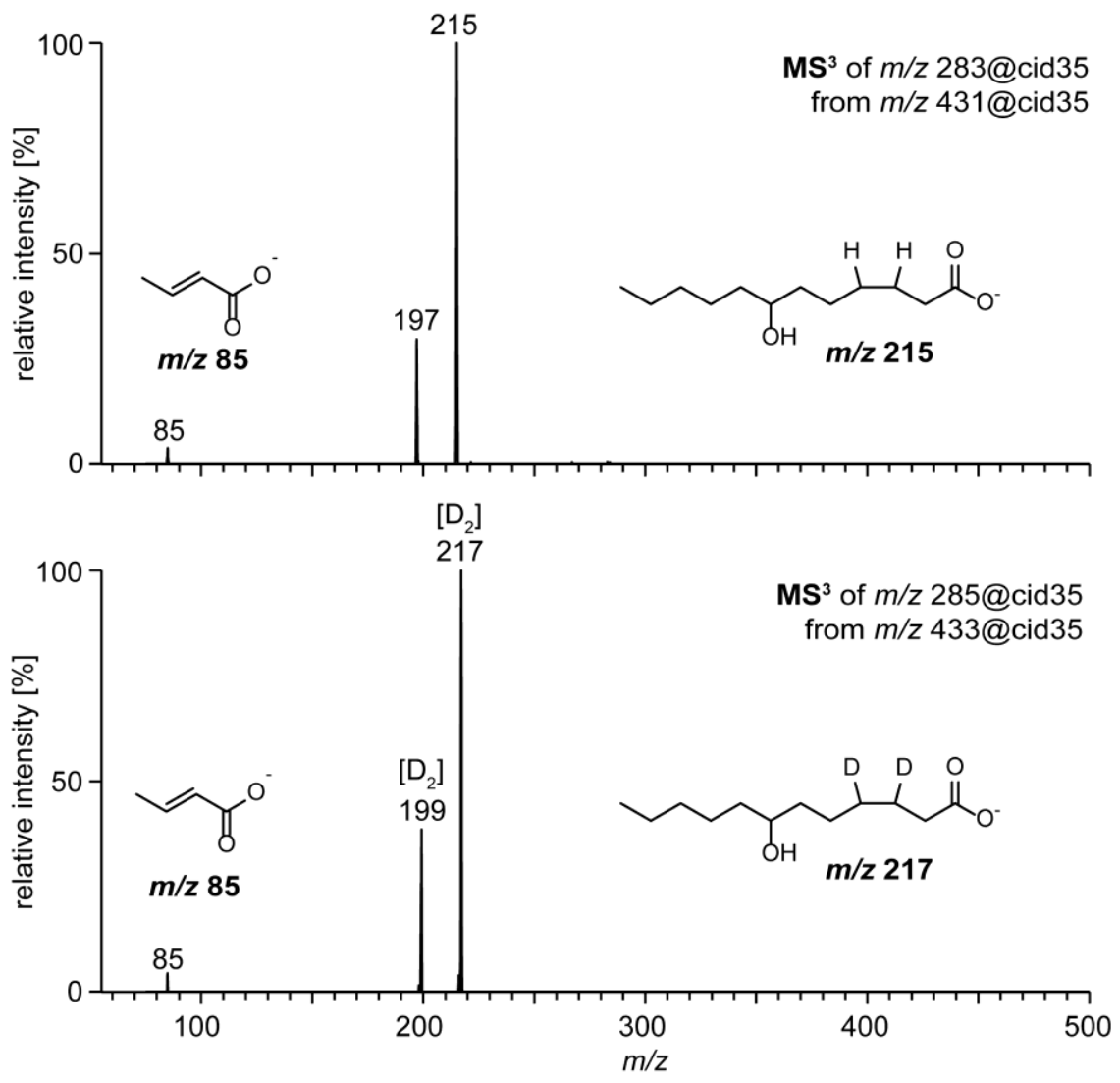


Figure S8c: Comparative analysis of ESI-(-)-MS ${ }^{4}$ spectra of semisynthetic asc-C4-C12 (fasc\#9) and $\left[3,4-D_{2}\right]$-asc-C4-C12 ([D $]$-fasc\#9) prepared by $P d / C$-catalyzed hydrogenation and deuterogenation of asc-C4-C12:1 (fasc\#2, 4) from C. remanei, respectively.

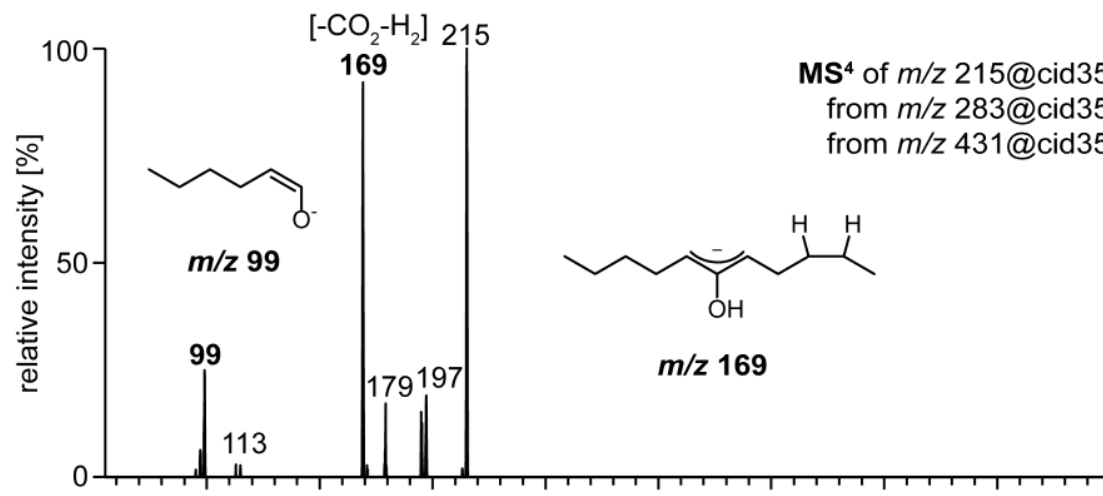

$\left[\mathrm{D}_{2}\right]$

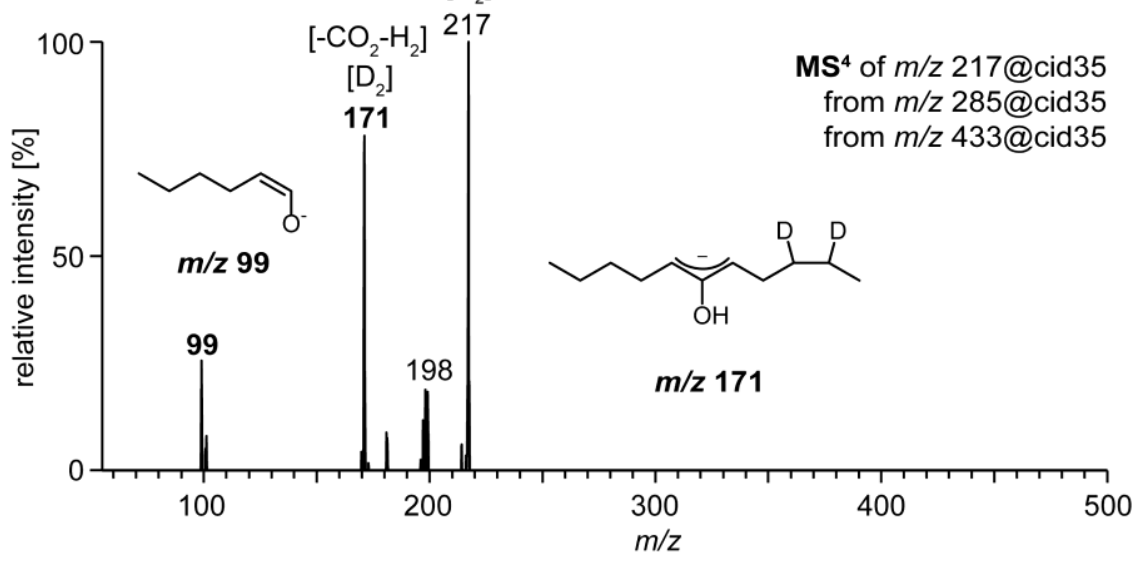


Figure S8d: Comparative analysis of ESI-(-)-MS ${ }^{5}$ spectra of semisynthetic asc-C4-C12 (fasc\#9) and $\left[3,4-D_{2}\right]$-asc-C4-C12 ([D $]$-fasc\#9) prepared by $P d / C$-catalyzed hydrogenation and deuterogenation of asc-C4-C12:1 (fasc\#2, 4) from C. remanei, respectively.
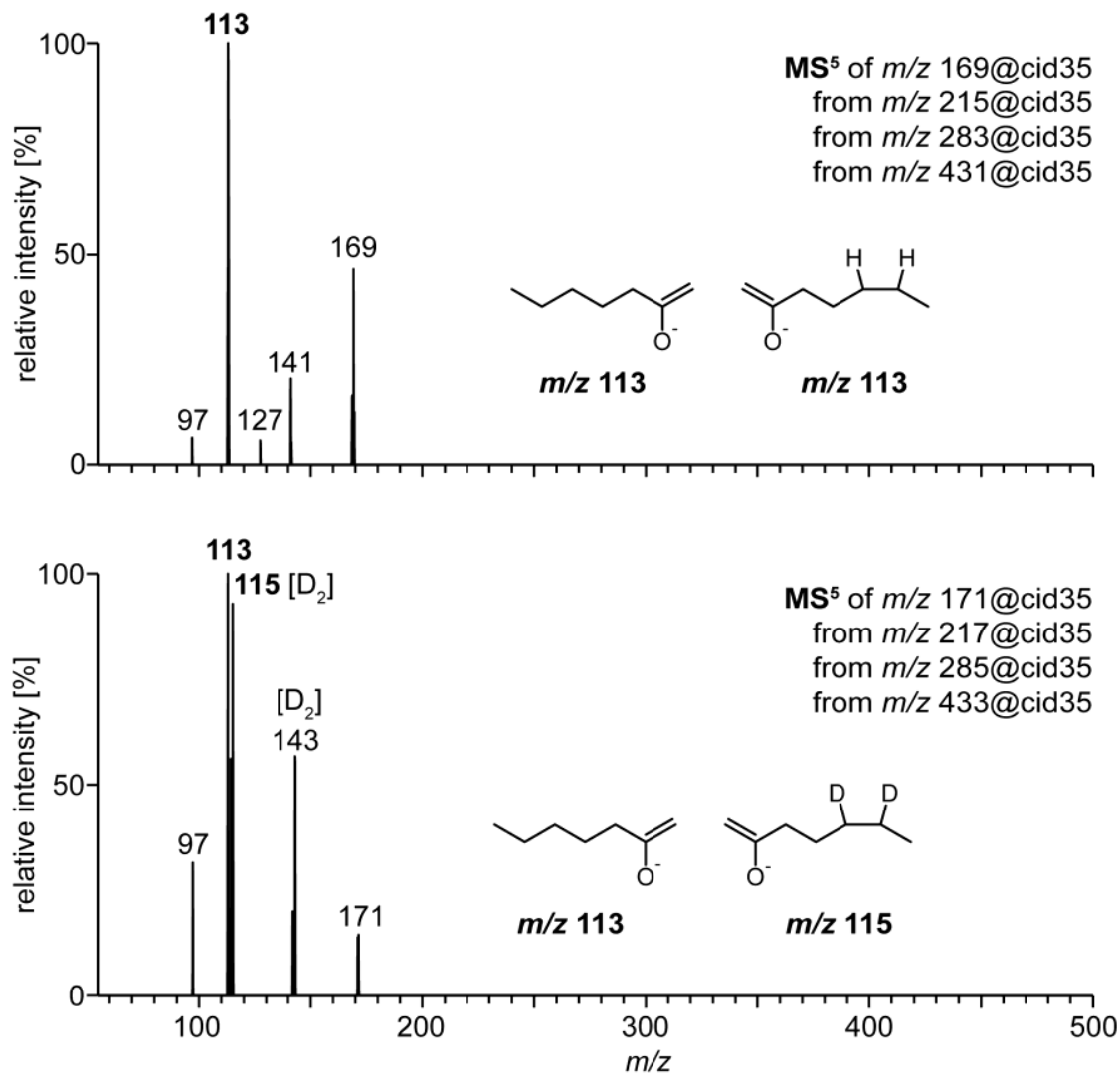
Figure S9a: HPLC-ESI-(-)-MS ${ }^{n}$ spectra asc-C4-(w-3)-C6 (fasc\#5) from C. latens.
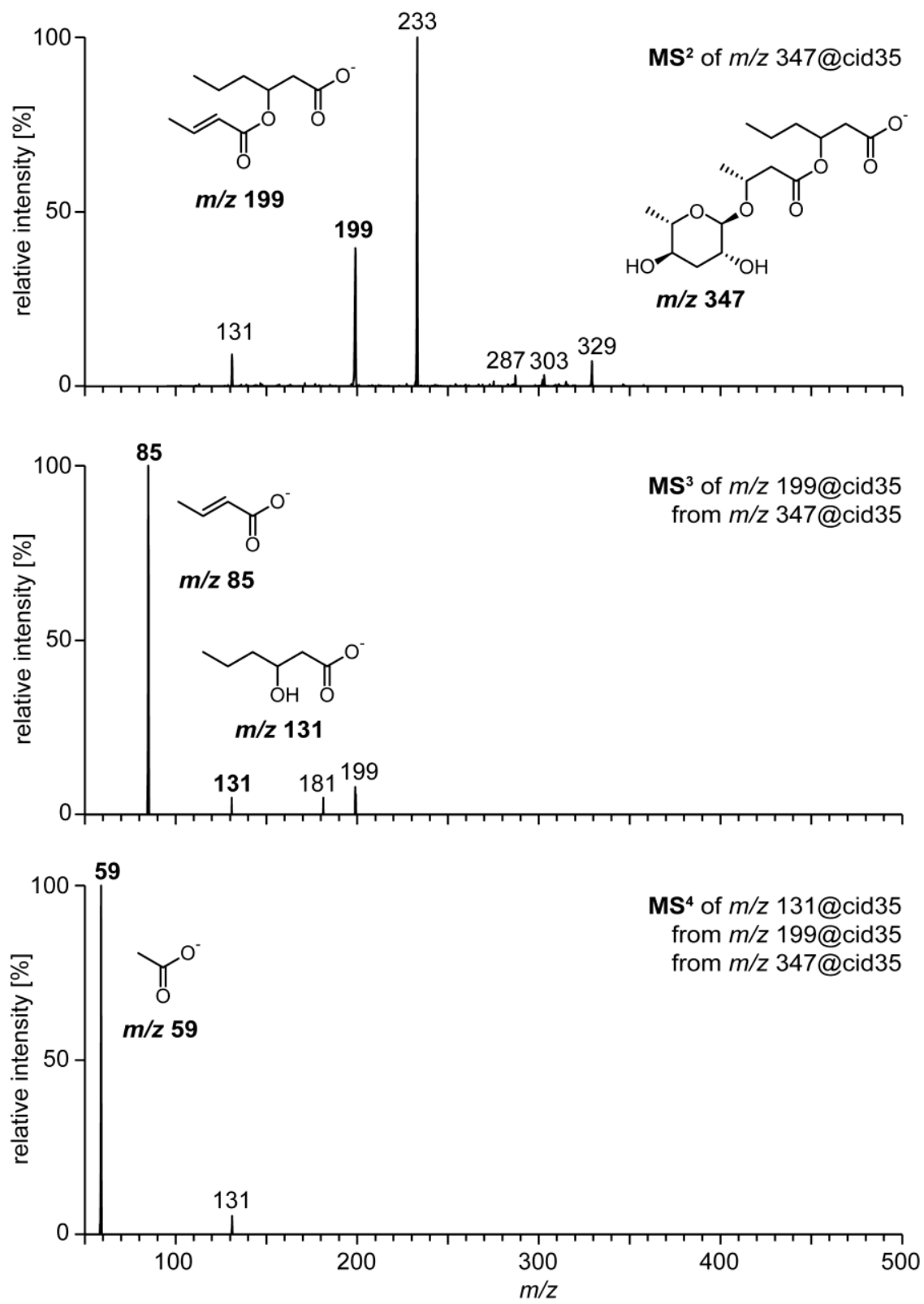
Figure S9b: HPLC-ESI-(-)-MS ${ }^{n}$ spectra asc-C4-(w-4)-C6 (fasc\#6) from C. latens.
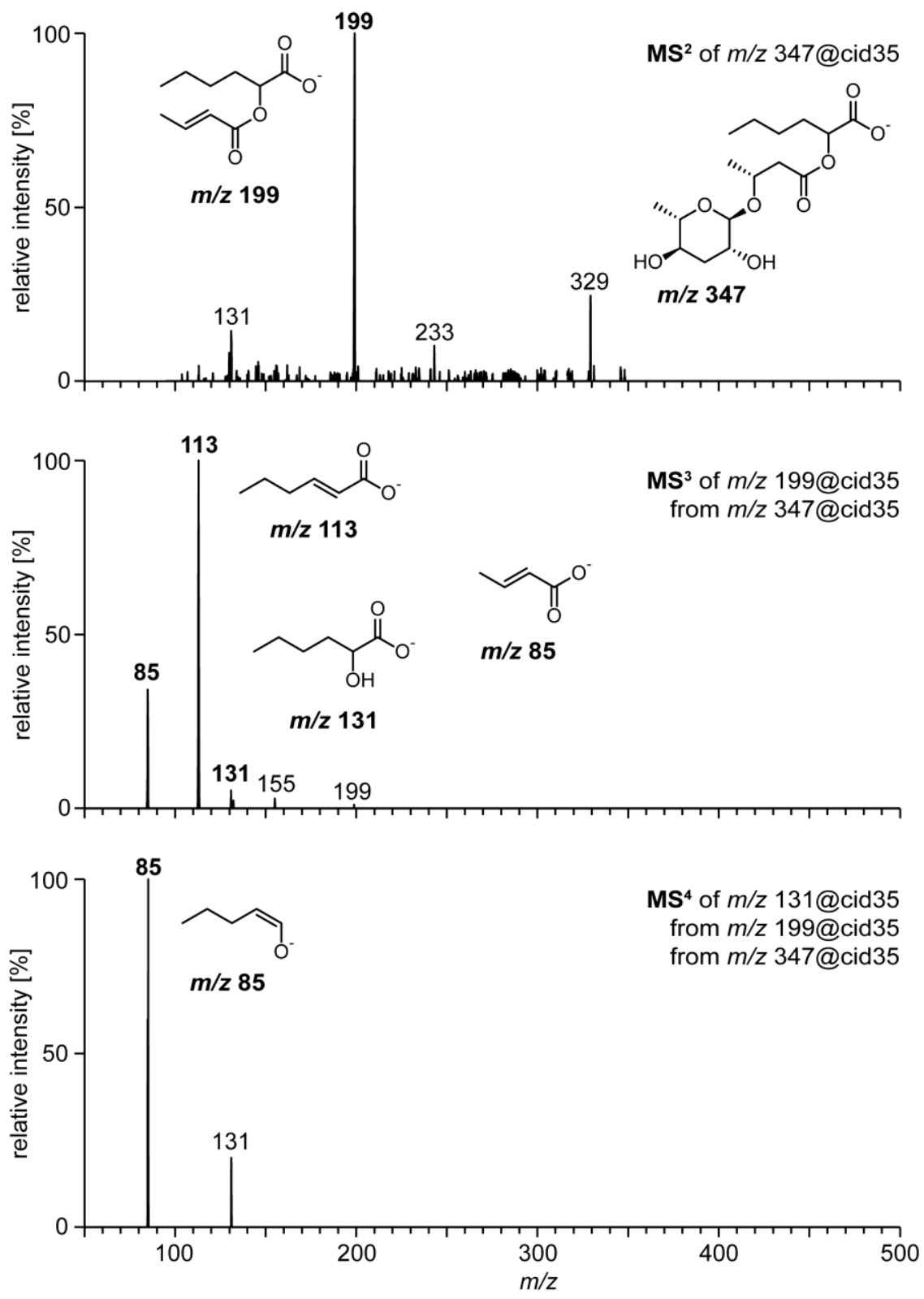
Figure S9c: HPLC-ESI-(-)-MS ${ }^{n}$ spectra asc-C4-( $\left.\omega-5\right)-C 8$ (fasc\#4, 7) from C. latens.
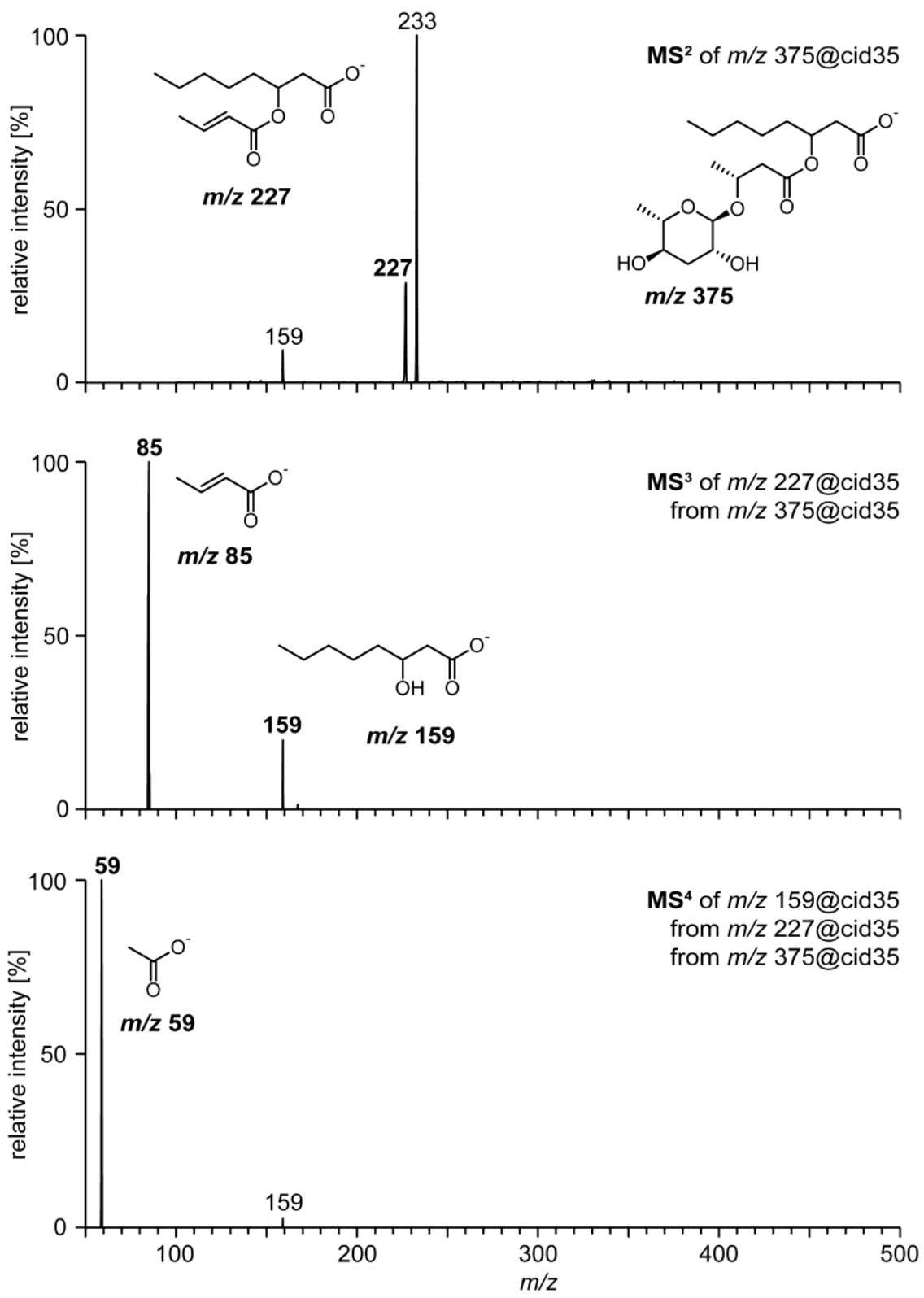
Figure S9d: HPLC-ESI-(-)-MS ${ }^{n}$ spectra asc-C4-(w-6)-C8 (fasc\#7) from C. latens.
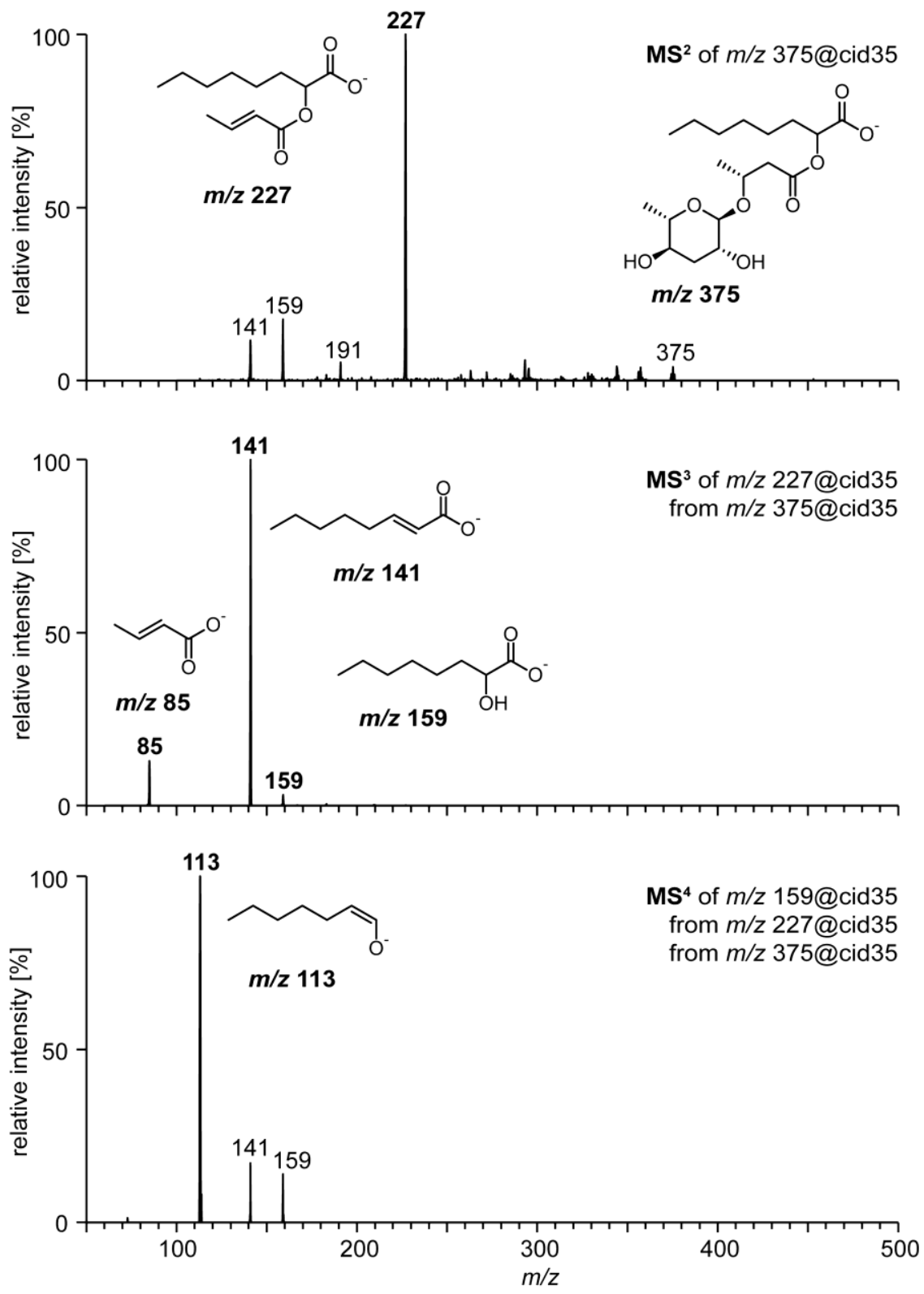
Figure S9e: HPLC-ESI-(-)-MS ${ }^{n}$ spectra of asc-C4-C10 (fasc\#8) from C. remanei.
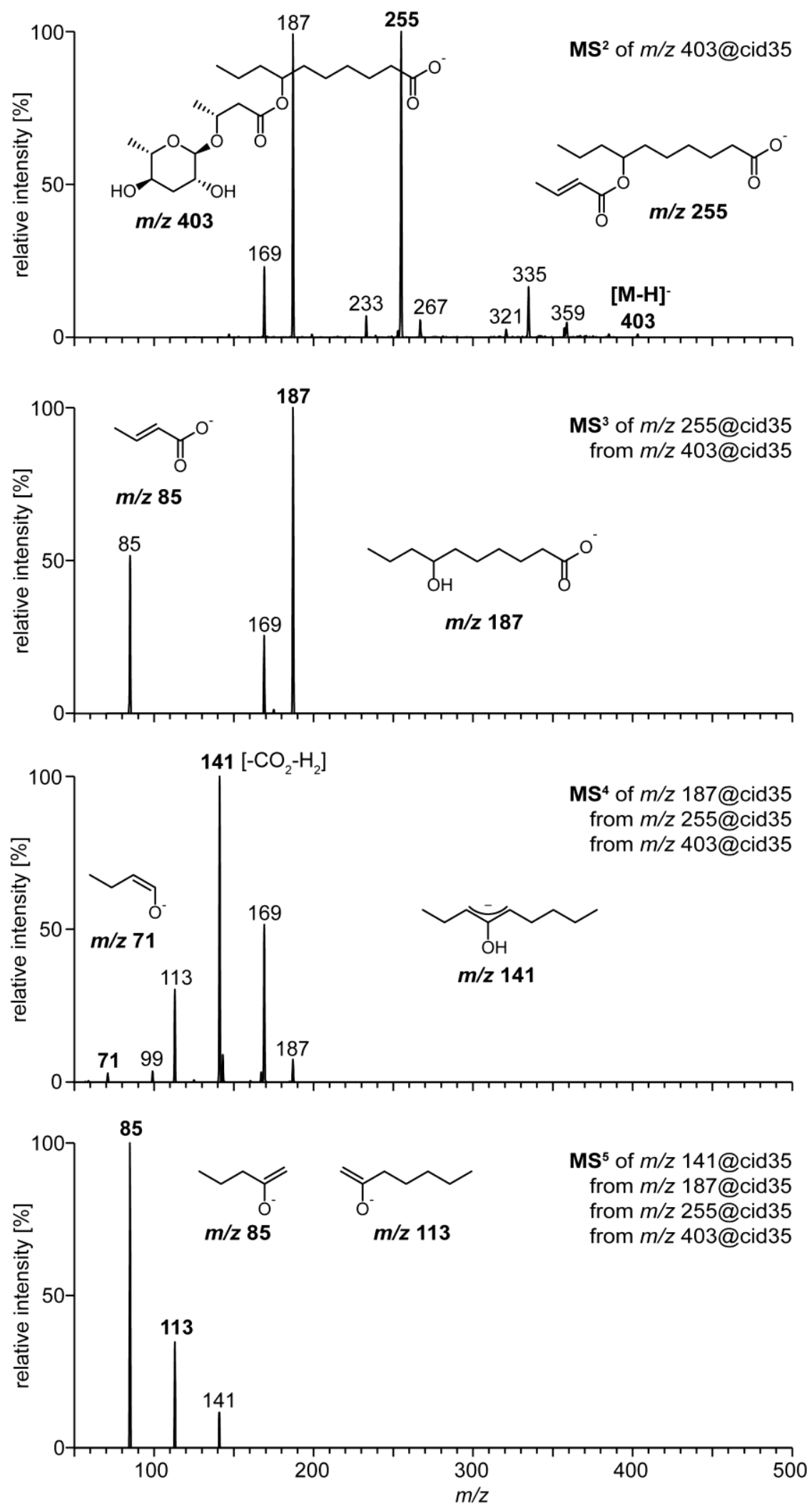
Figure S9f: HPLC-ESI-(-)-MSn spectra of asc-C4-C12 (fasc\#9) from C. remanei.
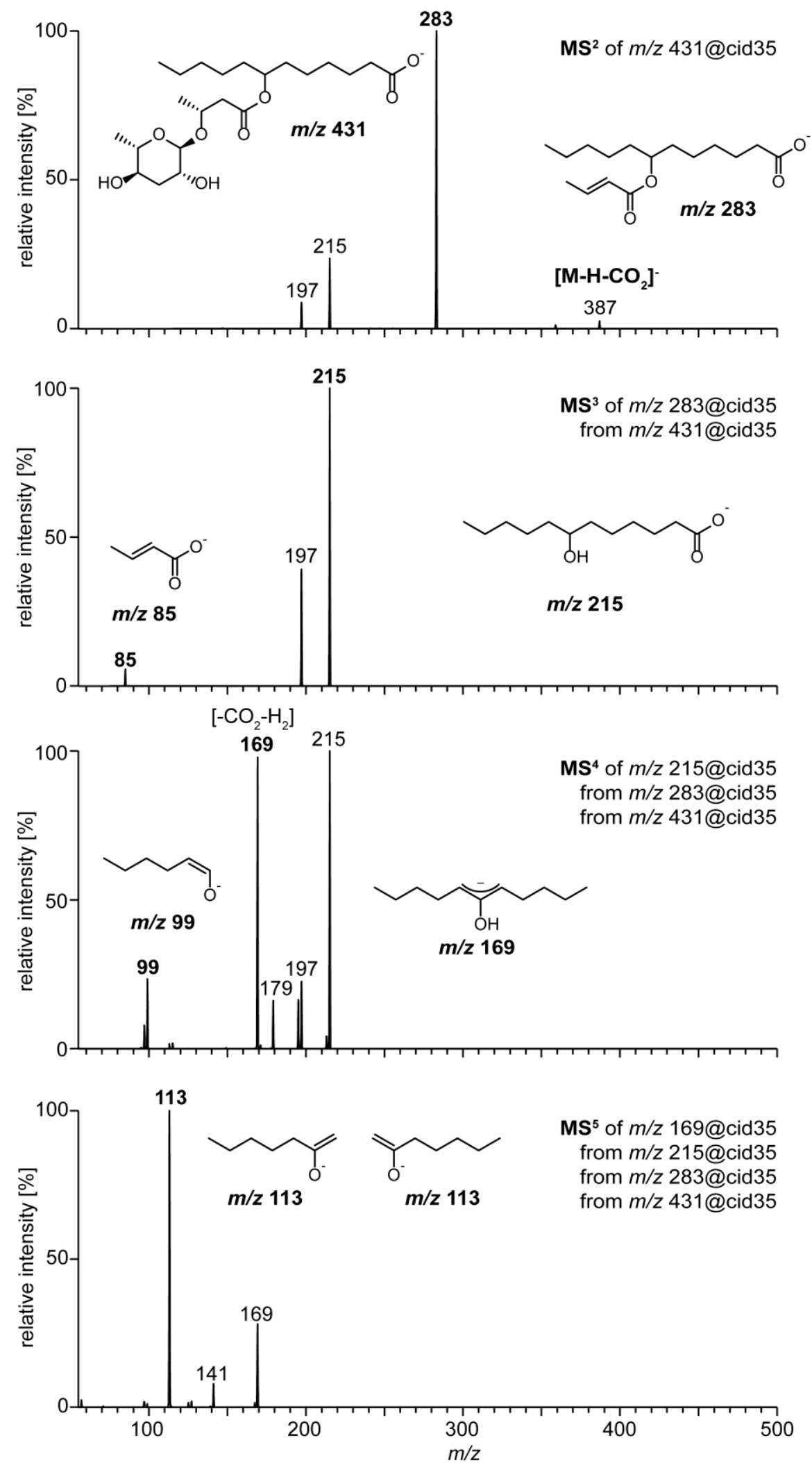
Figure S9g: HPLC-ESI-(-)-MS ${ }^{n}$ spectra of asc-C4-C13 (fasc\#10) from C. remanei.
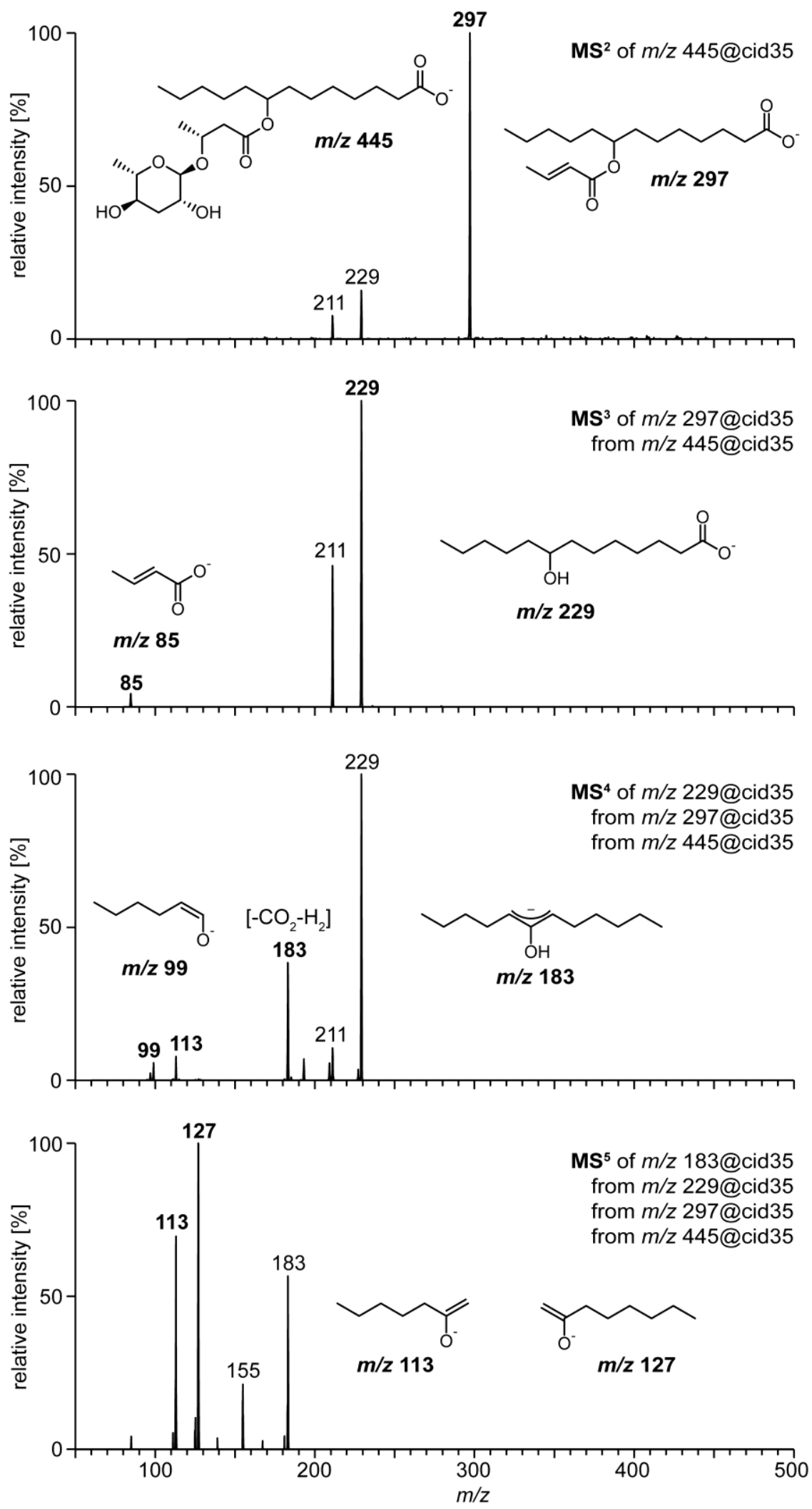
Figure S9h: HPLC-ESI-(-)-MS ${ }^{n}$ spectra of asc-C4-C14 (fasc\#11) and MS ${ }^{4}$ spectrum of asc-C4C16 (fasc\#12) from C. remanei.
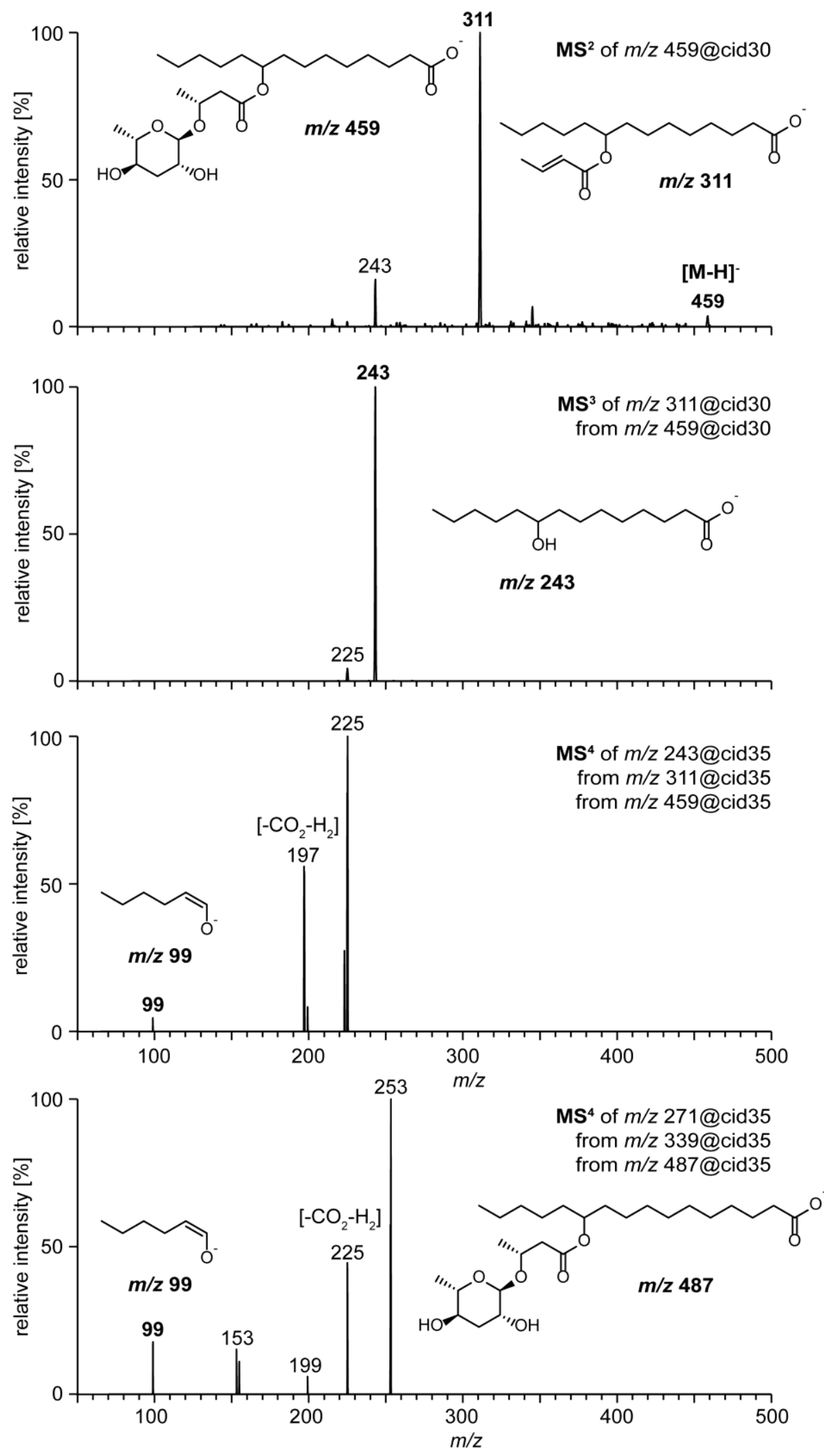
Figure S9i: HPLC-ESI-(-)-MS ${ }^{n}$ spectra of asc-C4-cyC10 (fasc\#13) from C. remanei.
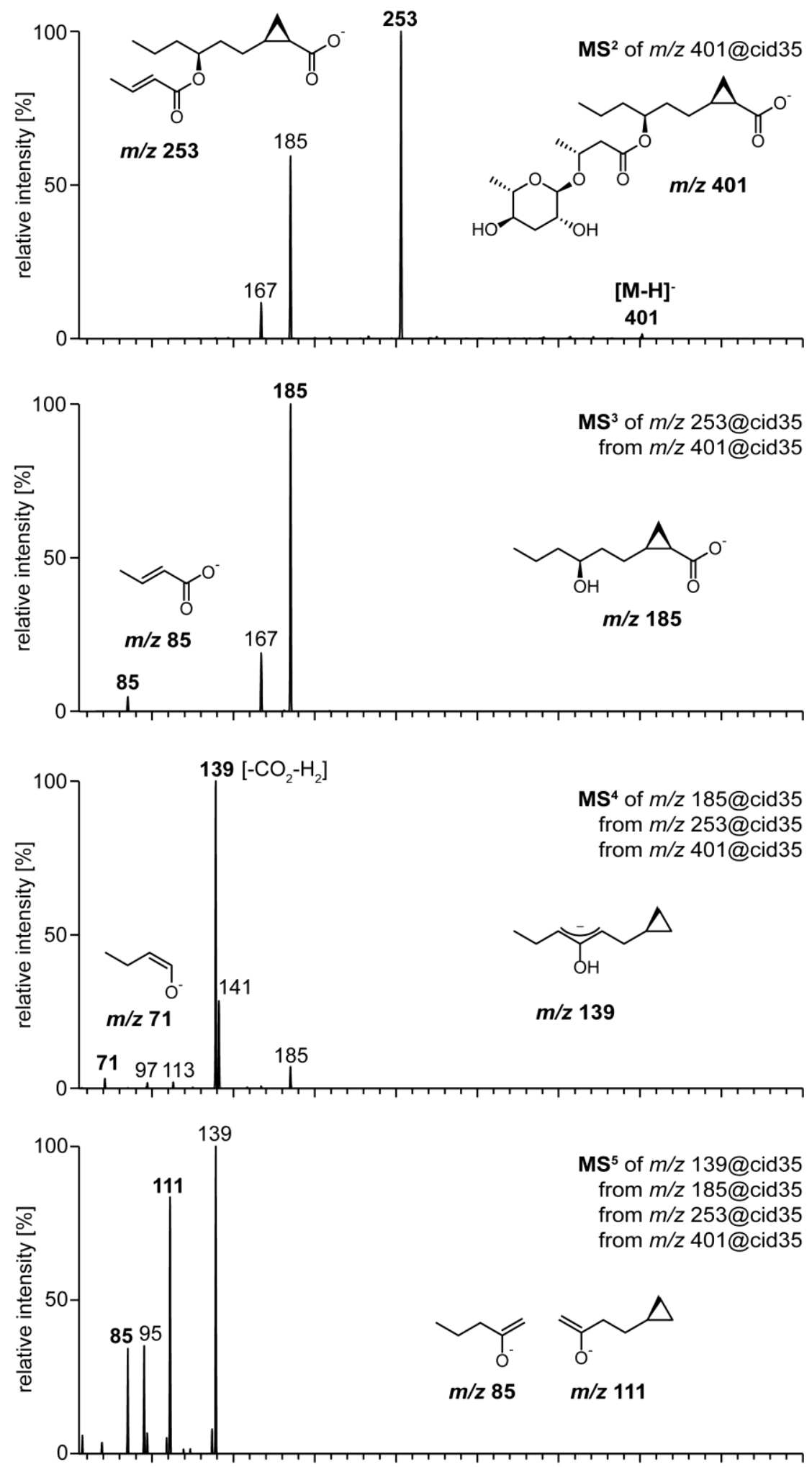
Figure S9j: HPLC-ESI-(-)-MS ${ }^{n}$ spectra of asc-C4-cyC11 (fasc\#1, 3) from C. remanei.
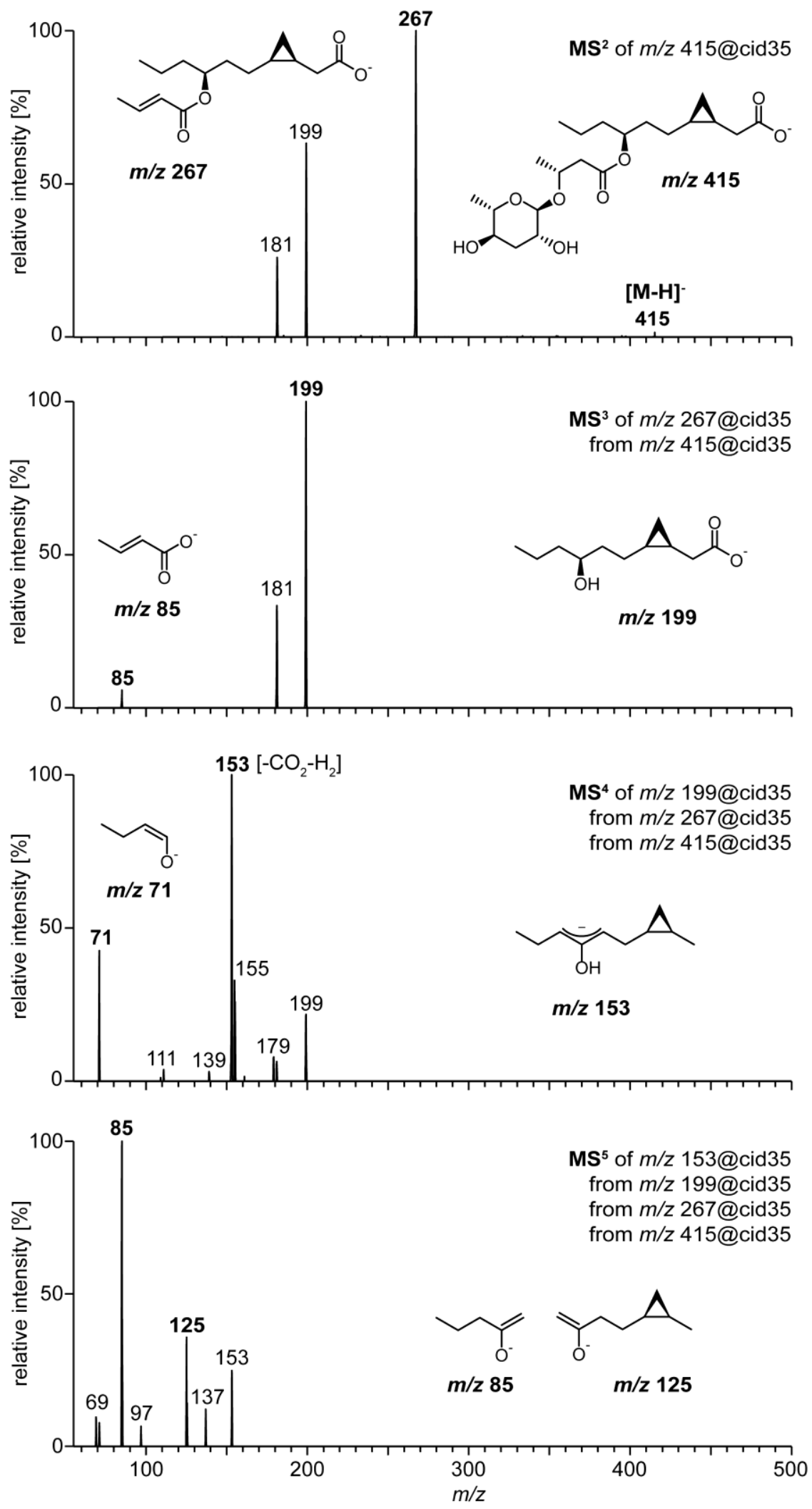
Figure S9k: HPLC-ESI-(-)-MS ${ }^{n}$ spectra of asc-C4-cyC13 (fasc\#14) from C. remanei.
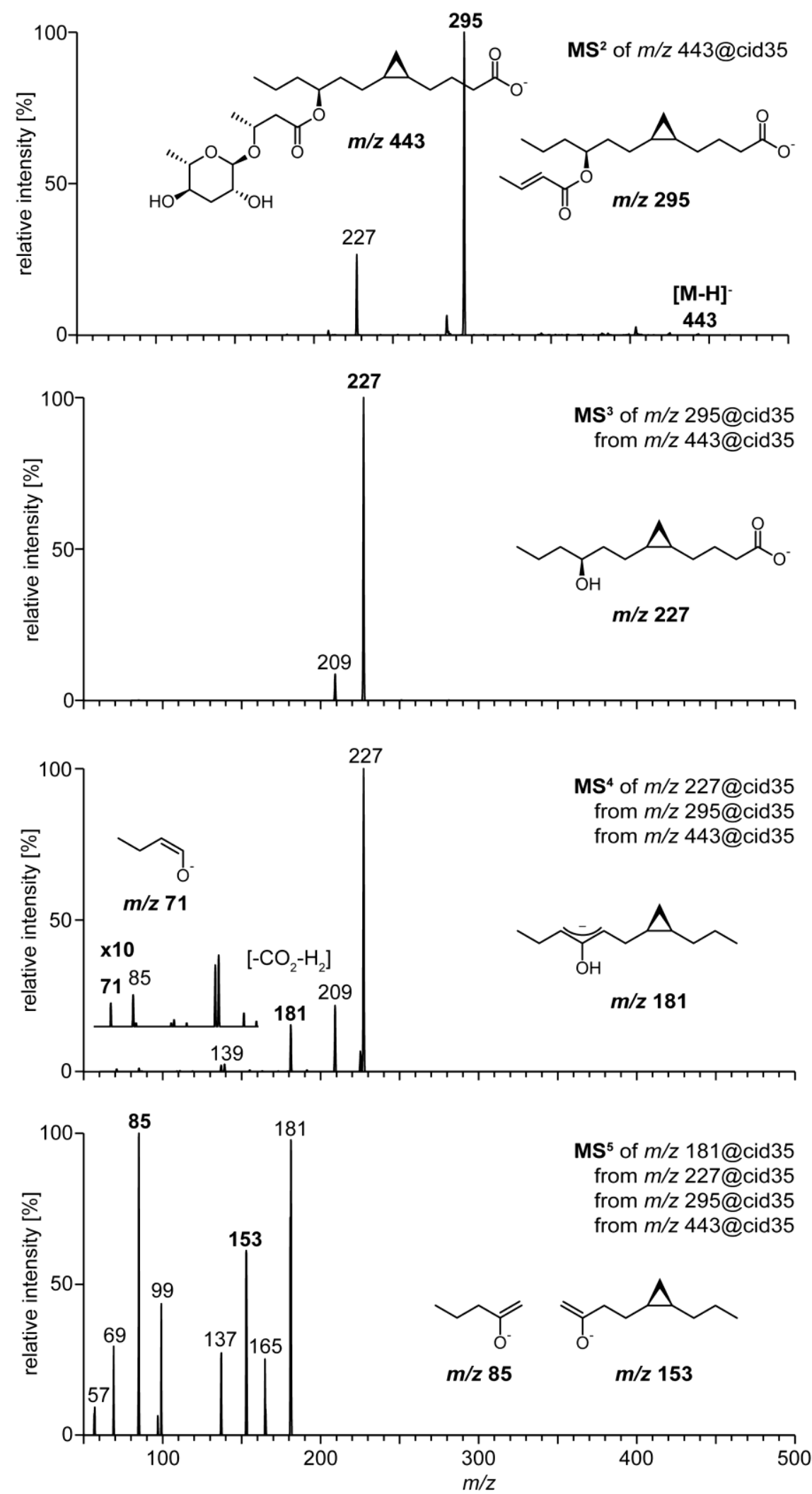
Figure S9I: HPLC-ESI-(-)-MS ${ }^{n}$ spectra of asc-C4-C10:1 (fasc\#3, 5) from C. remanei.
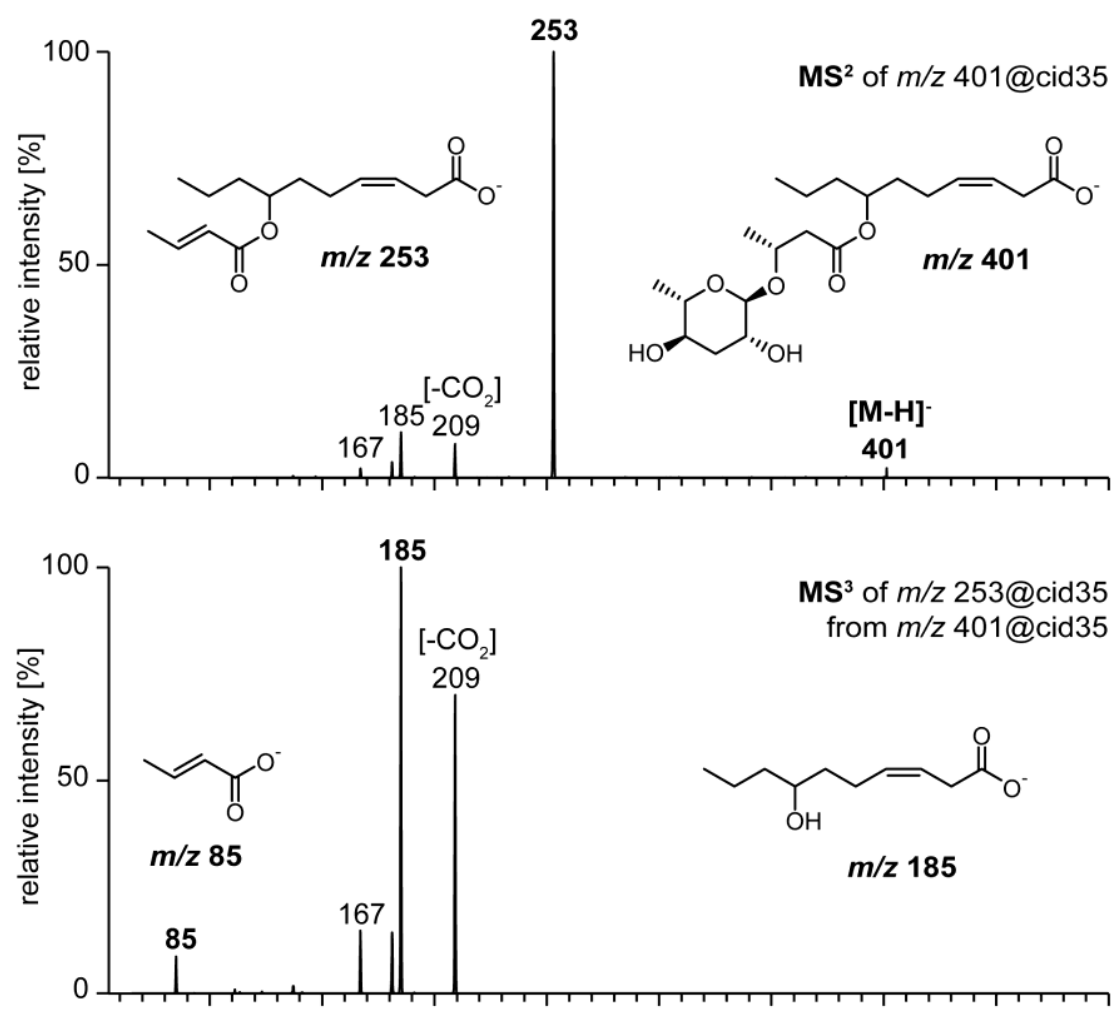

MS $^{3}$ of $m / z$ 253@cid35

from m/z 401@cid35

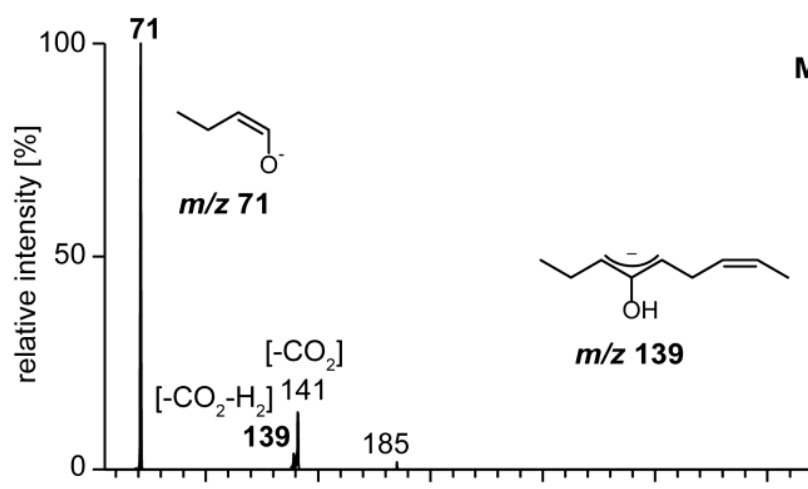

MS4 of $m / z$ 185@cid35 from m/z 253@cid35 from m/z 401@cid35

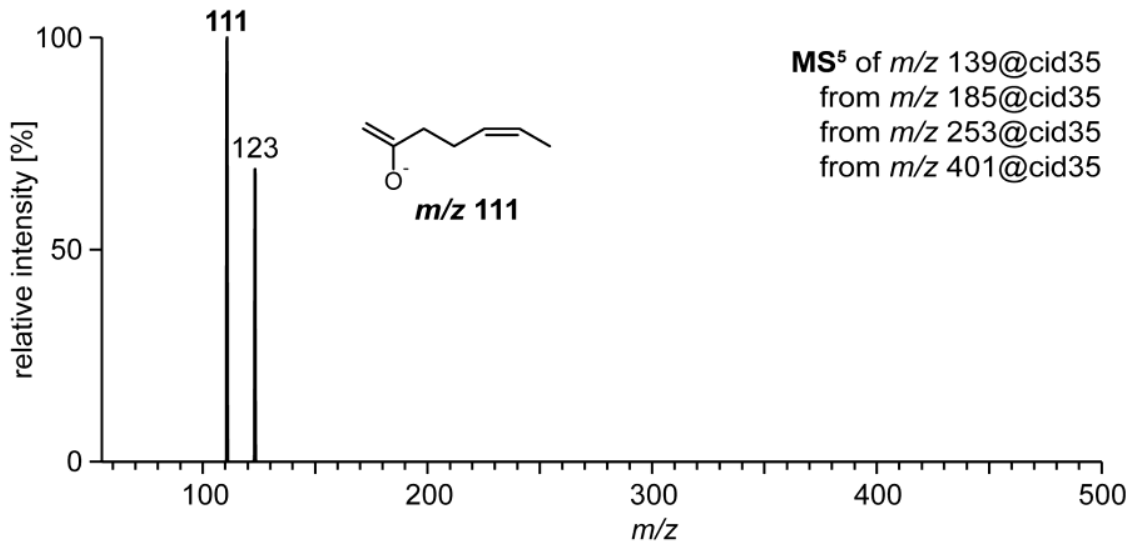


Figure S9m: HPLC-ESI-(-)-MS ${ }^{n}$ spectra of asc-C4-C12:1 (fasc\#2, 4) from C. remanei.
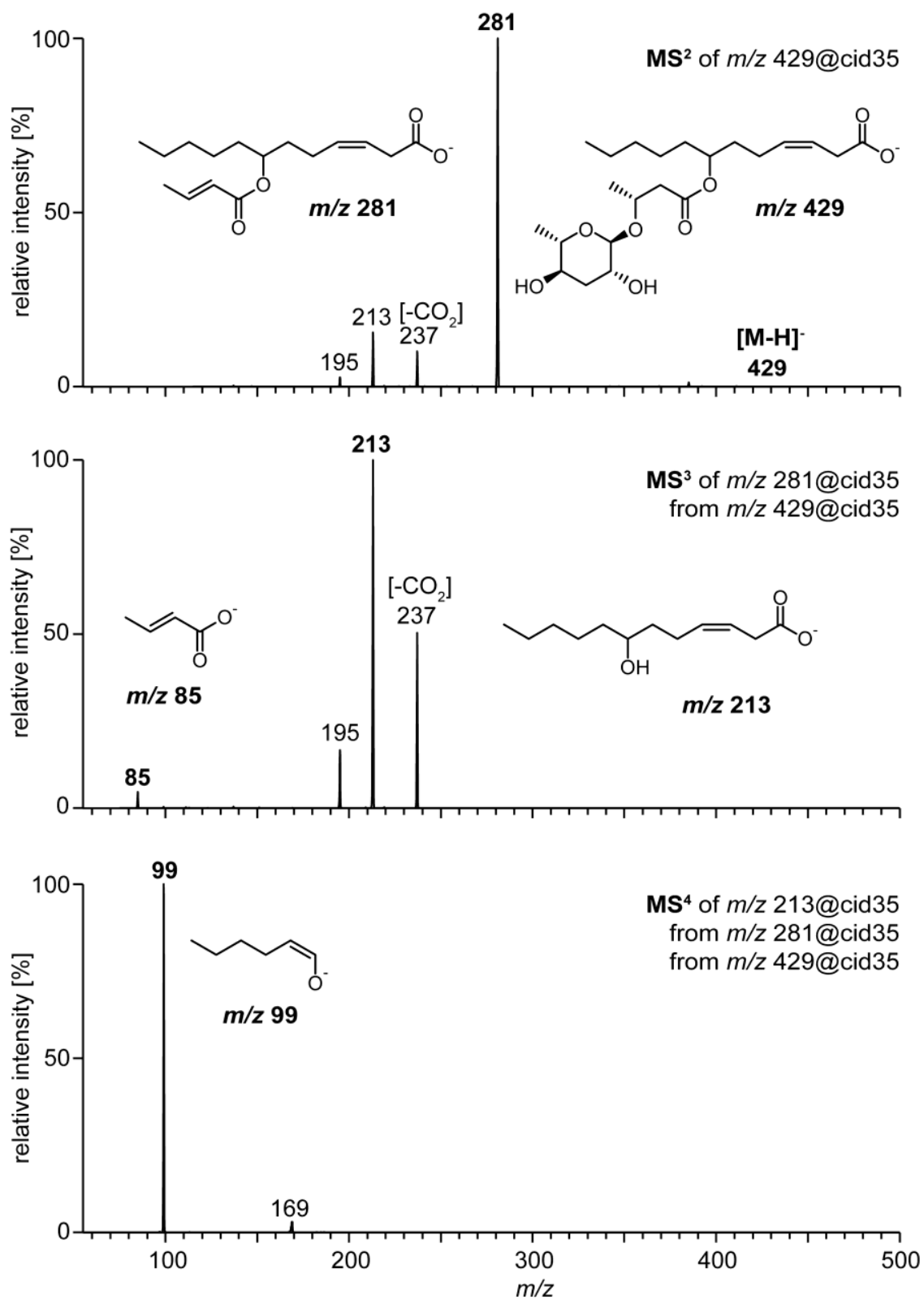
Figure S9n: HPLC-ESI-(-)-MS ${ }^{n}$ spectra of asc-C4-C14:1 (fasc\#16) from C. remanei.
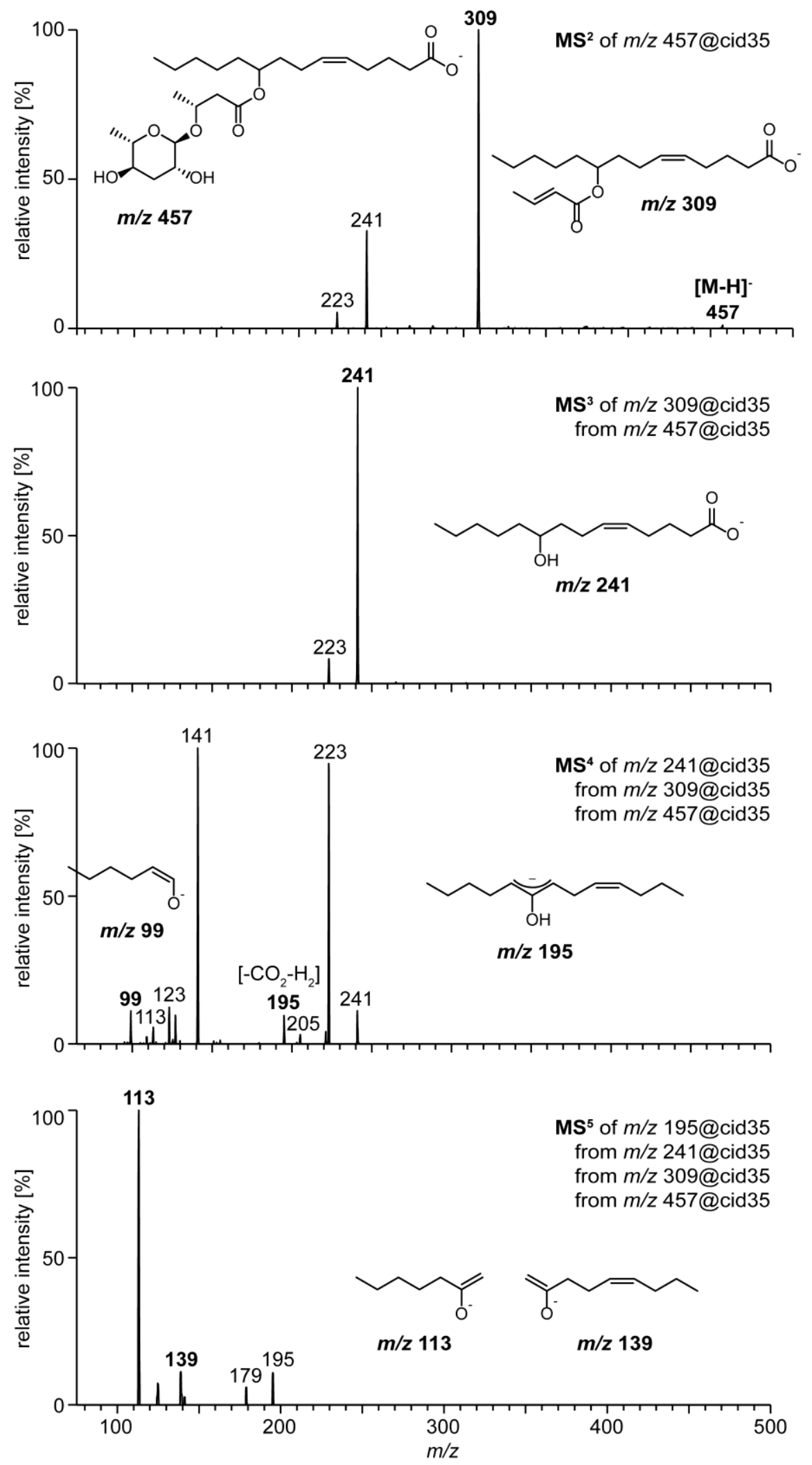
Figure S9o: HPLC-ESI-(-)-MS ${ }^{n}$ spectra of asc-C4-C16:1 (fasc\#17) from C. remanei.
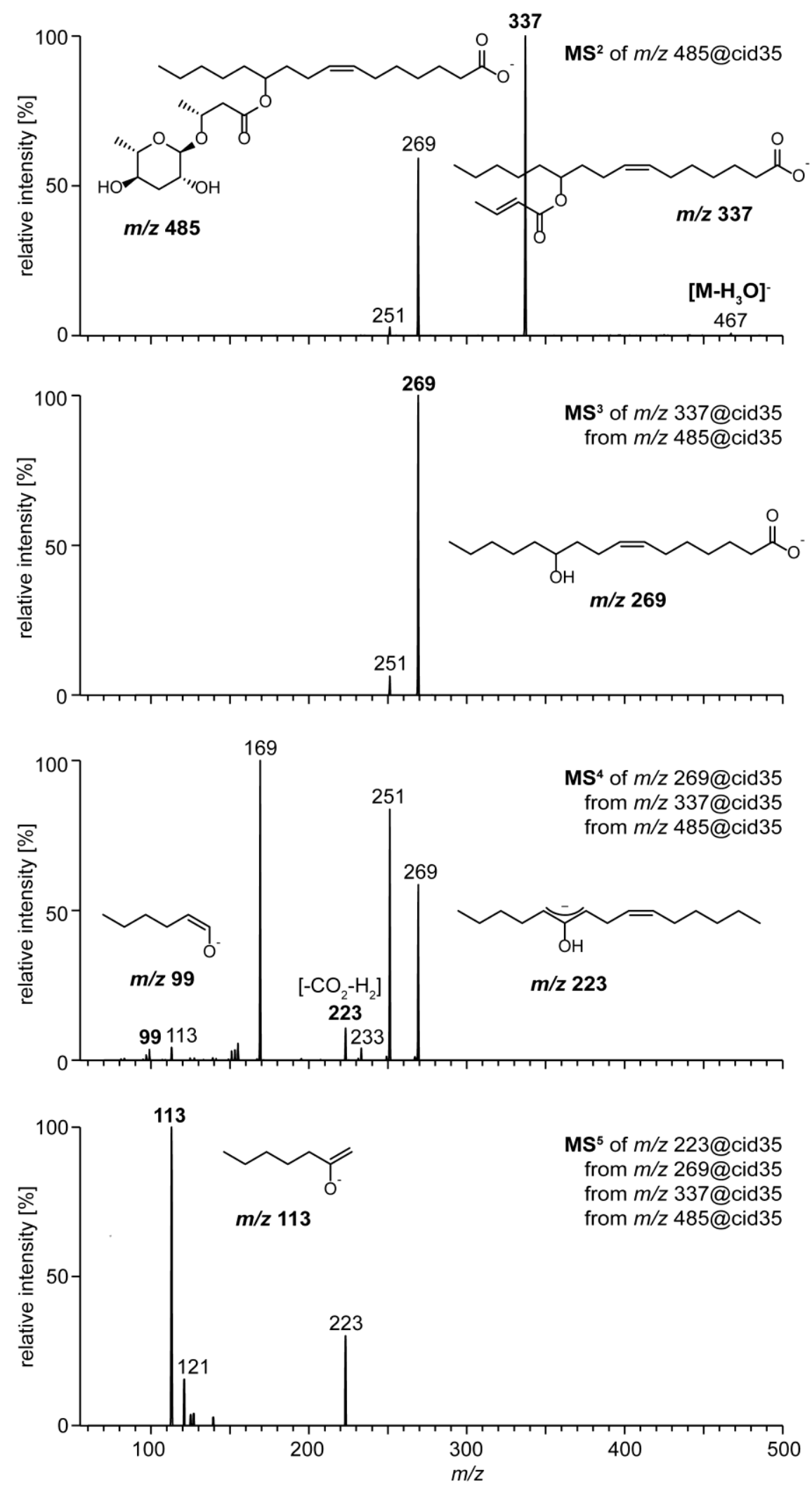
Figure S9p: HPLC-ESI-(-)-MS ${ }^{n}$ spectra of asc-C4-(w-3)-C12:2 (fasc\#18) from C. remanei.
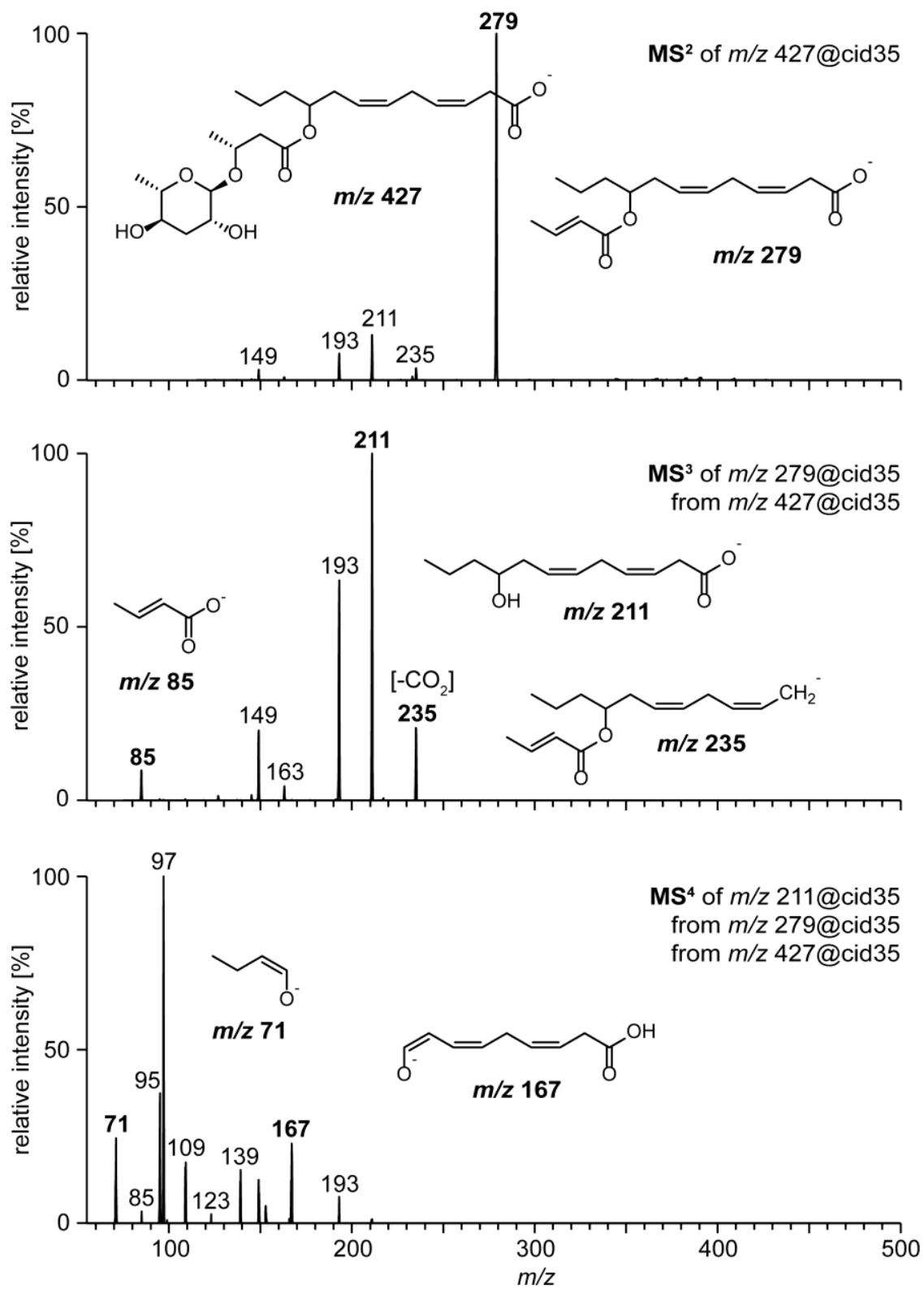
Figure S9q: HPLC-ESI-(-)-MS ${ }^{n}$ spectra of asc-C4-( $\left.\omega-5\right)-C 12: 2$ (fasc\#19) from C. remanei.
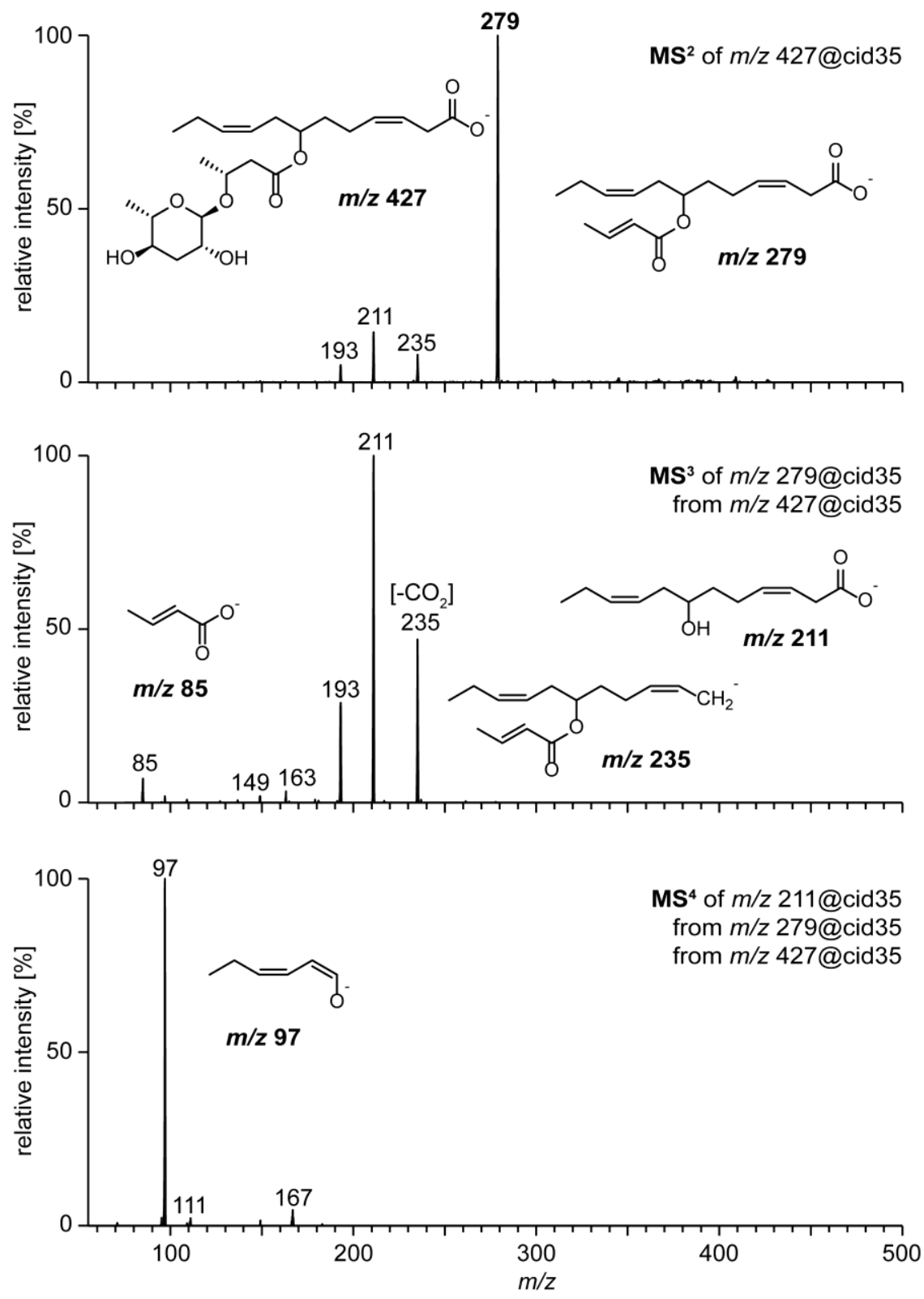
Figure S9r: HPLC-ESI-(-)-MS ${ }^{n}$ spectra of semisynthetic asc-C4-( $\left.\omega-5\right)-C 12$ (fasc\#2) obtained by hydrogenation of asc-C4-( $\omega-5)-C 12: 2$ (fasc\#19) from $C$. remanei.
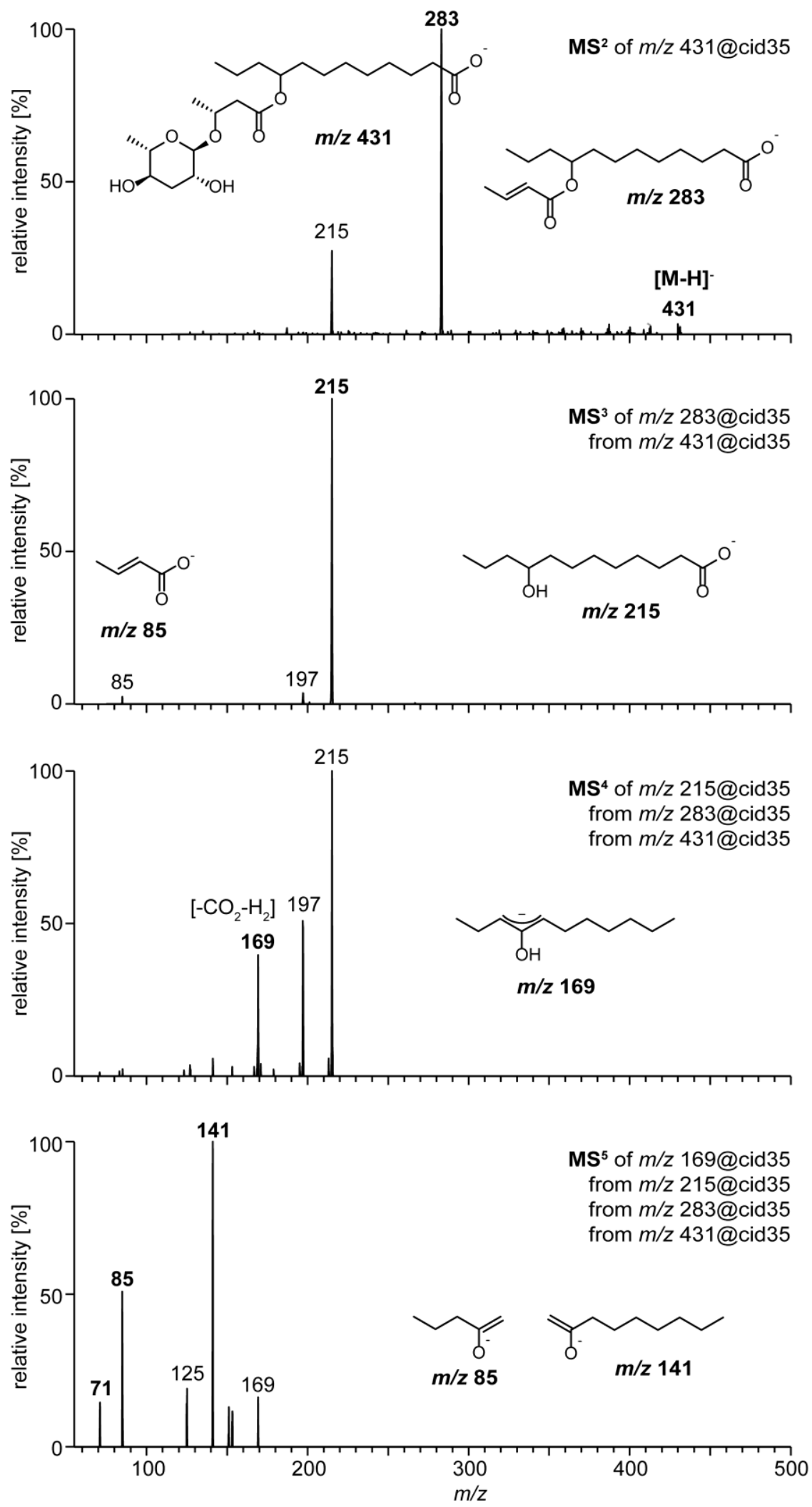
Figure S9s: HPLC-ESI-(-)-MS ${ }^{n}$ spectra of semisynthetic asc-C4-( $\left.\omega-3\right)-C 12$ obtained by hydrogenation of asc-C4-( $\omega-3)-C 12: 2$ (fasc\#18) from $C$. remanei.
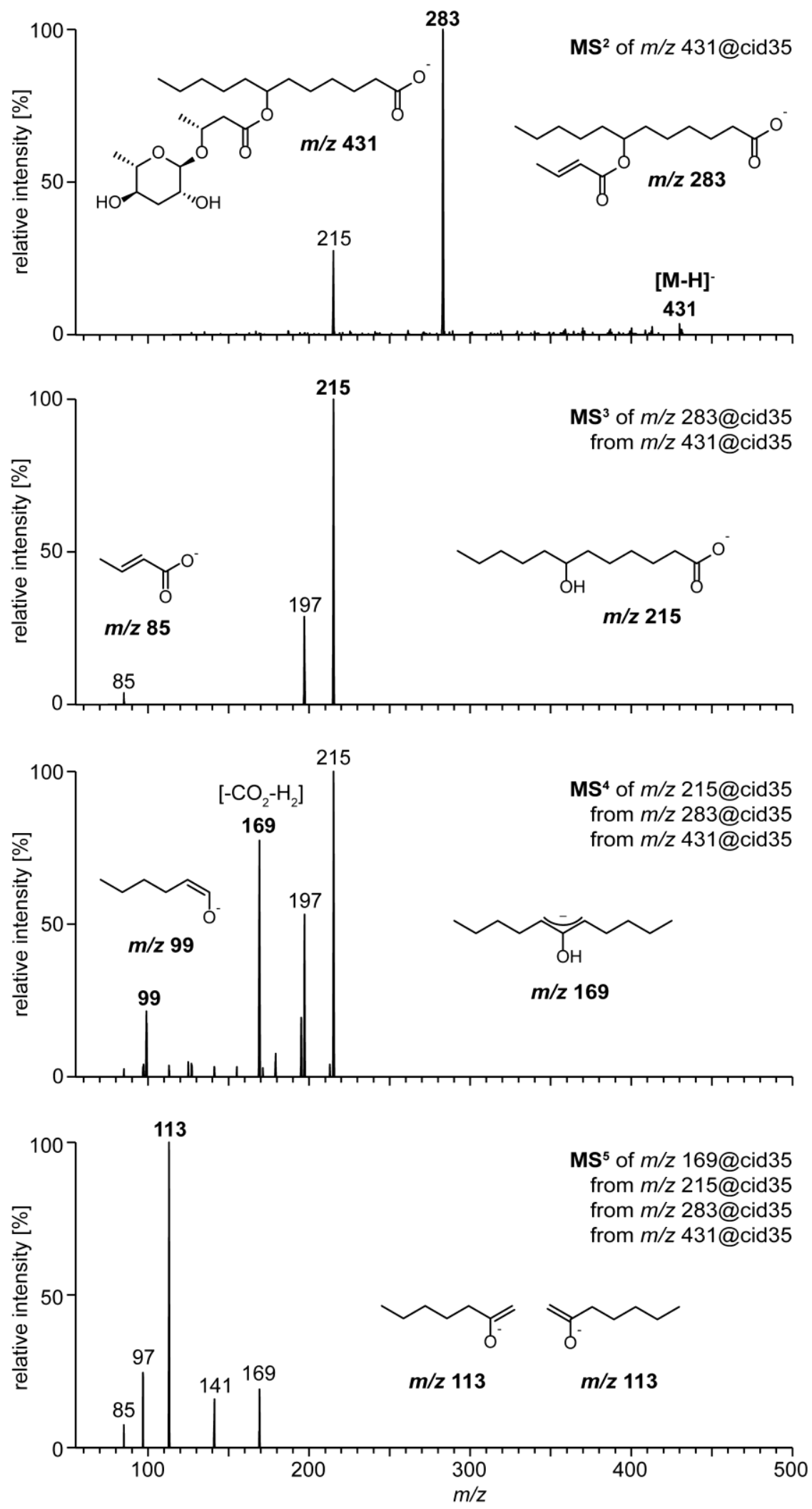
Figure S10a: HPLC-MS ${ }^{n}$ spectra of semisynthetic asc-C4-(3,4-OH)-C10 obtained by $\mathrm{OsO}_{4}{ }^{-}$ catalyzed vicinal dihydroxylation of asc-C4-C10:1 (fasc\#3, 5) from C. remanei.
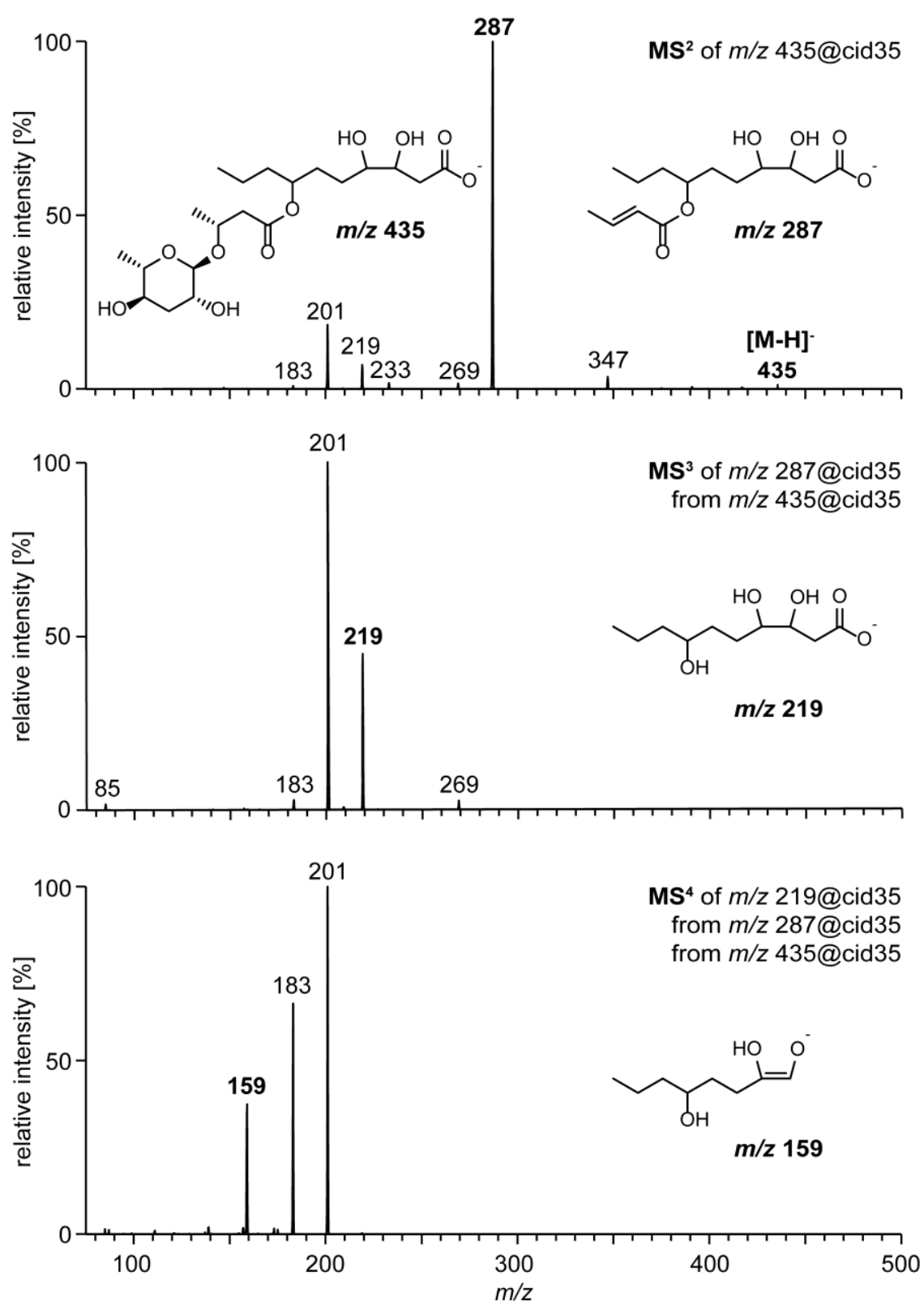
Figure S10b: HPLC-MS ${ }^{n}$ spectra of semisynthetic asc-C4-(3,4-OH)-C12 obtained by $\mathrm{OsO}_{4}$ catalyzed vicinal dihydroxylation of asc-C4-C12:1 (fasc\#2, 4) from C. remanei.
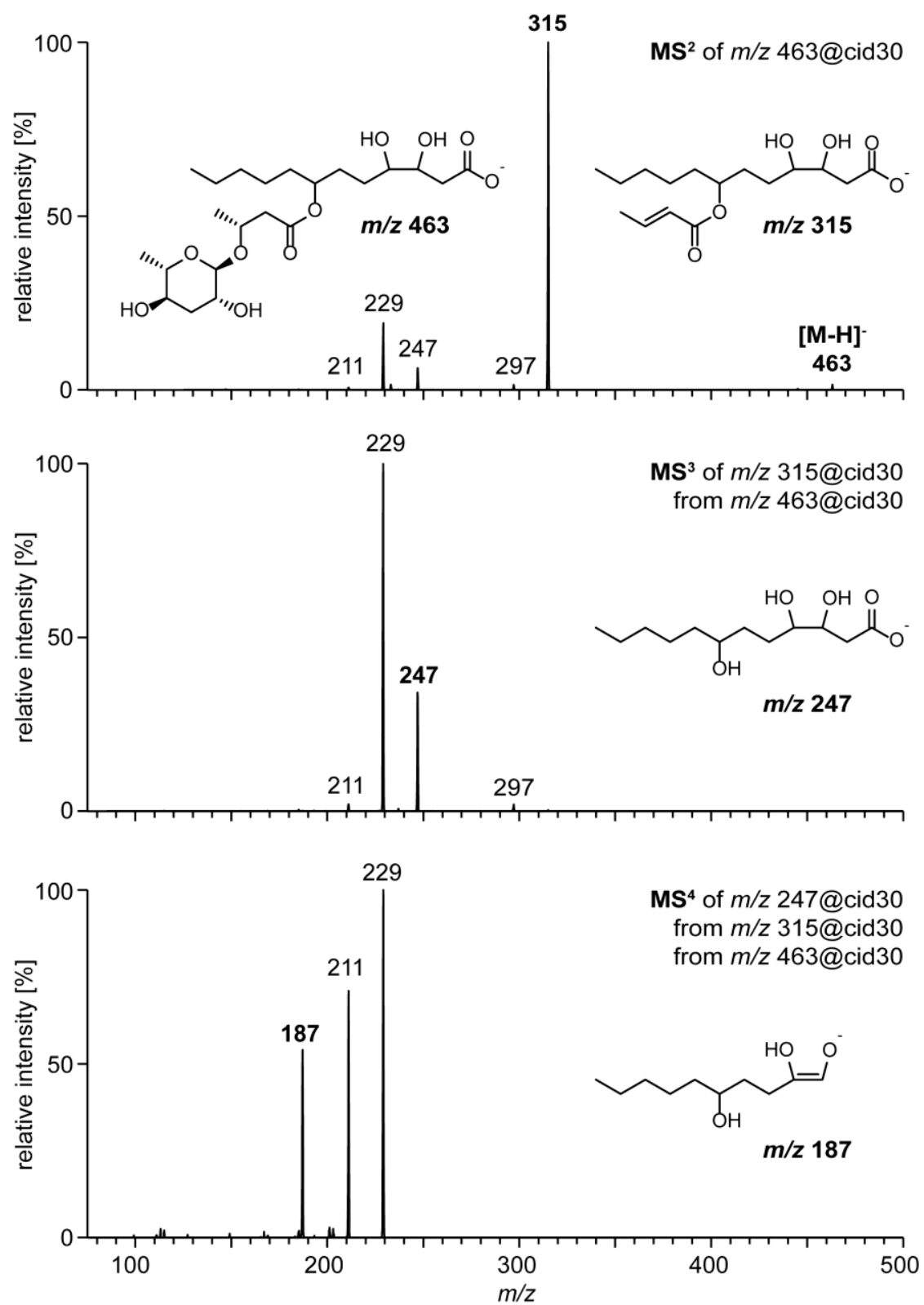
Figure S10c: HPLC-MS ${ }^{n}$ spectra of semisynthetic asc-C4-(5,6-OH)-C14 obtained by $\mathrm{OsO}_{4}{ }^{-}$ catalyzed vicinal dihydroxylation of asc-C4-C14:1 (fasc\#16) from C. remanei.
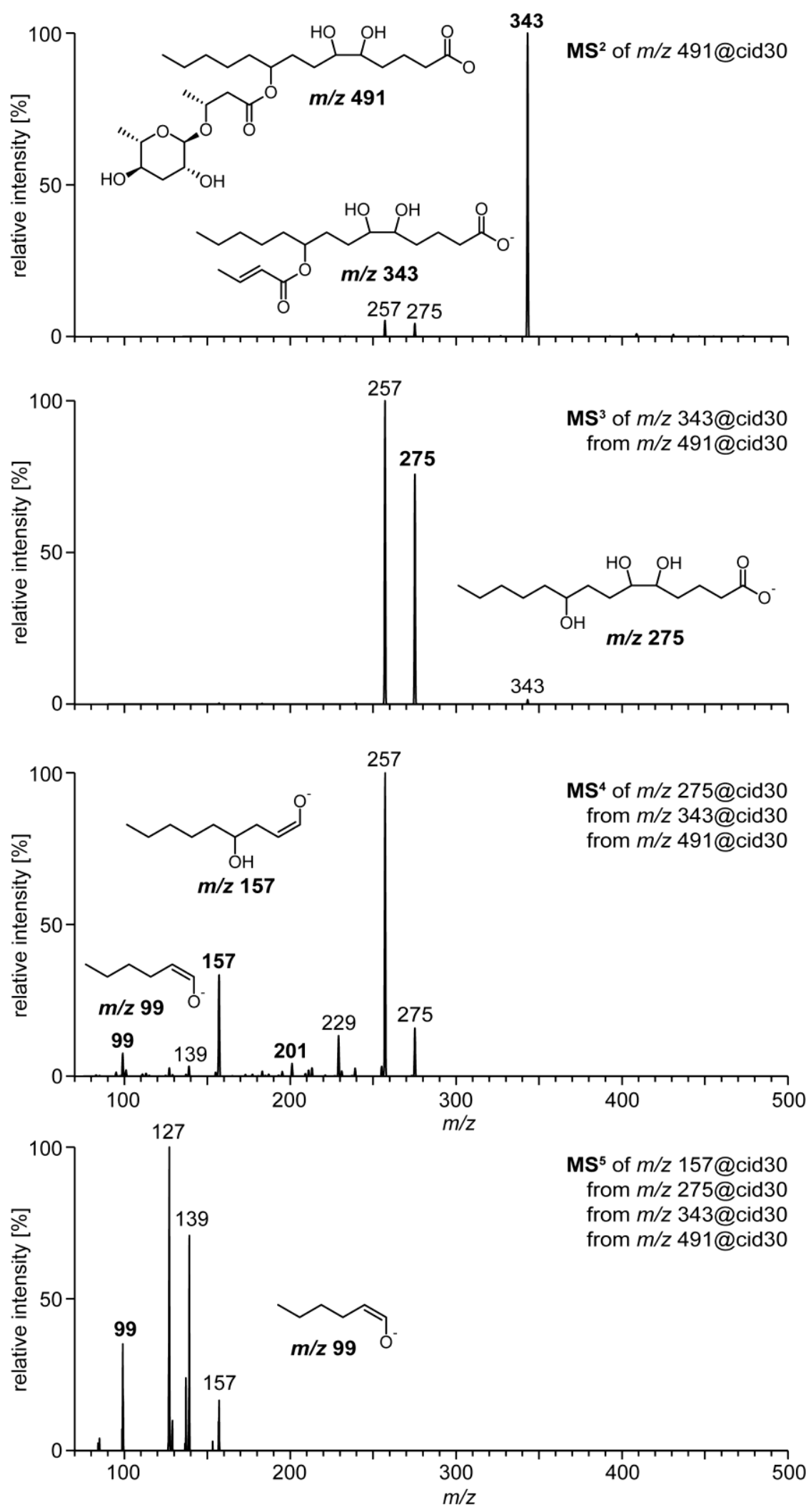
Fig. S11: Fatty acid ascaroside composition in exometabolomes of wild type isolates of Caenorhabditis remanei and its sister species Caenorhabditis latens (mean, $\mathrm{n}=3, \pm \mathrm{SEM}$ ).
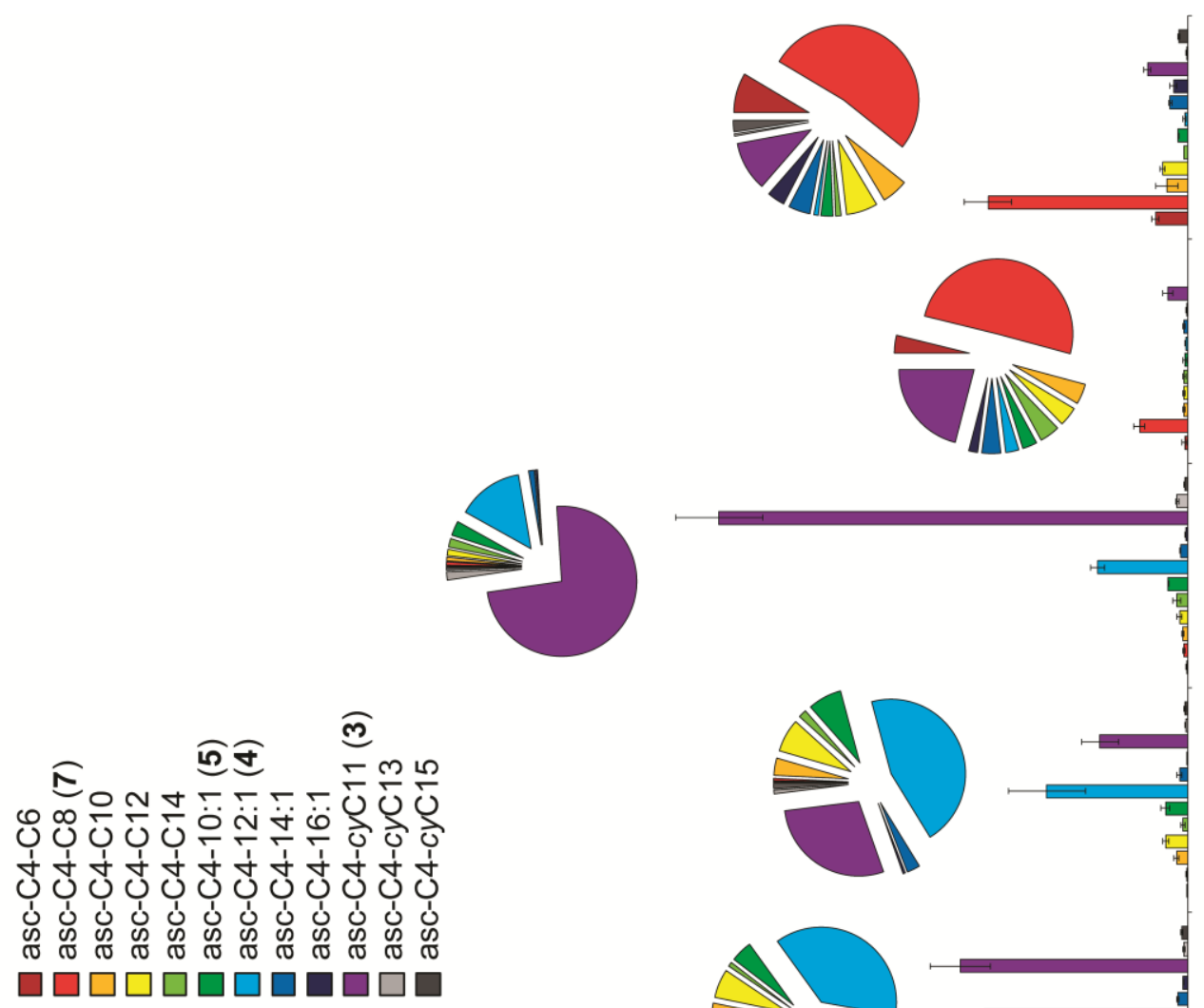

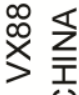

$\frac{\frac{2}{d}}{0}$

志志
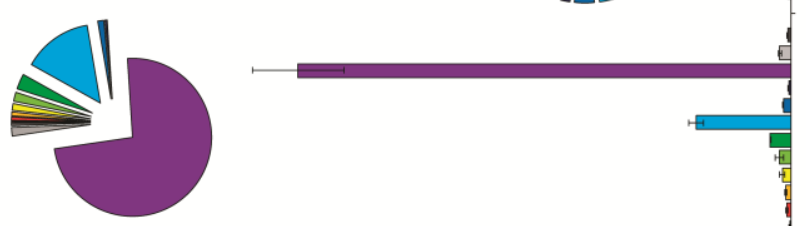

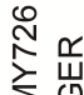

$\sum 0$
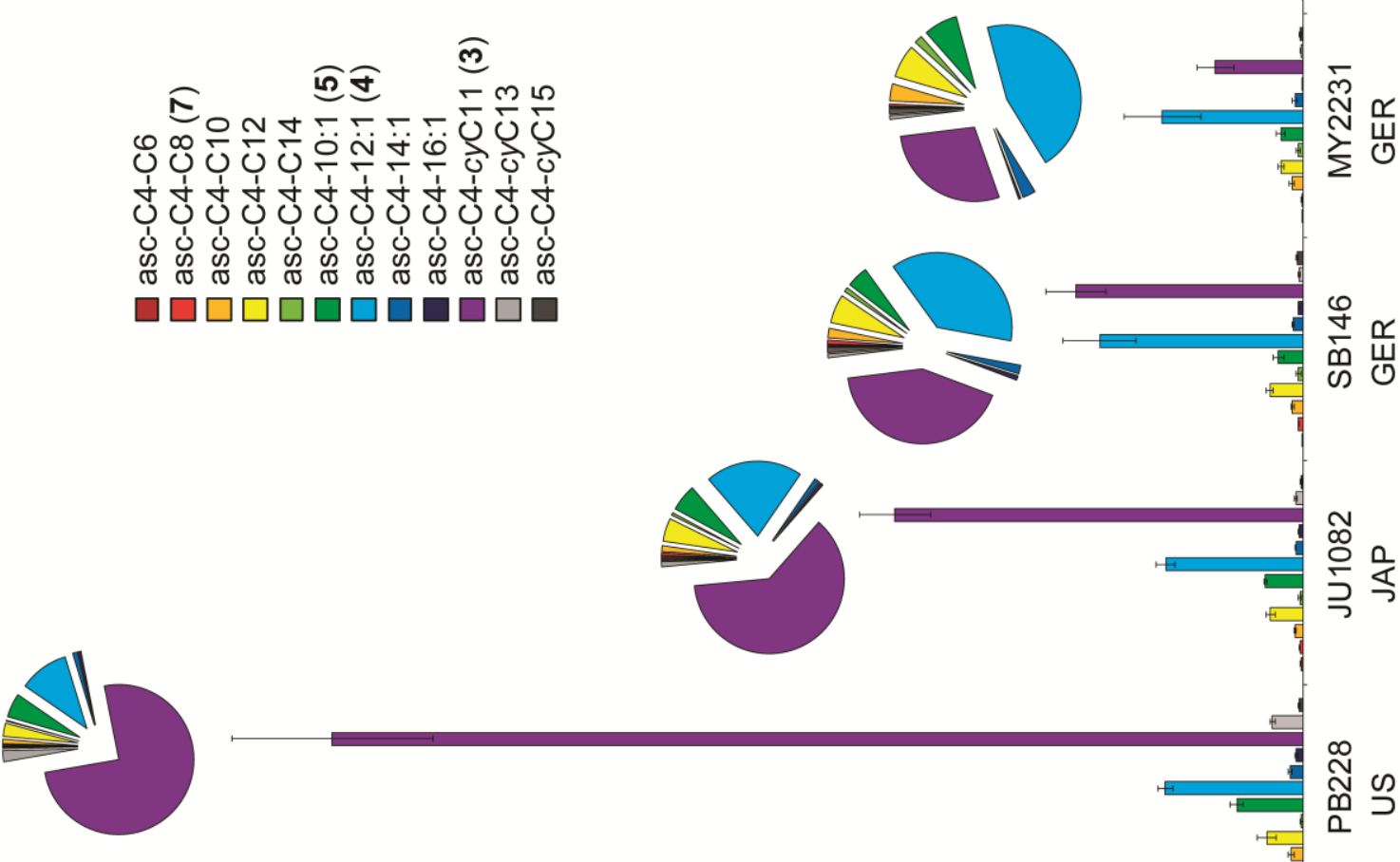

ลิ $\frac{\mathbb{x}}{4}$ $\gtrless$

\begin{tabular}{ll}
0 \\
\hline 1
\end{tabular}

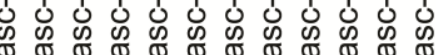

口ロ $\square \square \square \square \square \square \square \square \square$

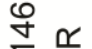

战

ఏ

ن

을 足
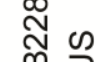

กั

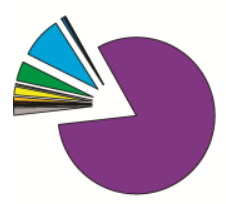

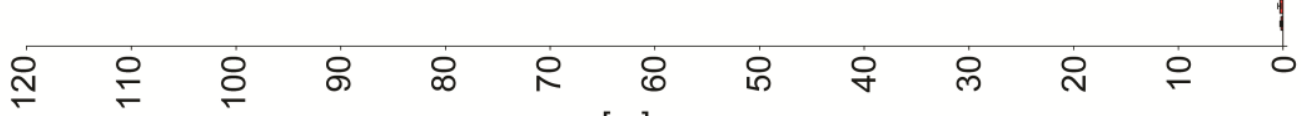

[\%] słunome әм!̣е|әл 
Figure S12: Phylogeny of Caenorhabditis species ${ }^{[9]}$ investigated by HPLC-ESI-(-)-MS/MS precursor ion screening. Fatty acid ascaroside (fasc) containing species marked in red.

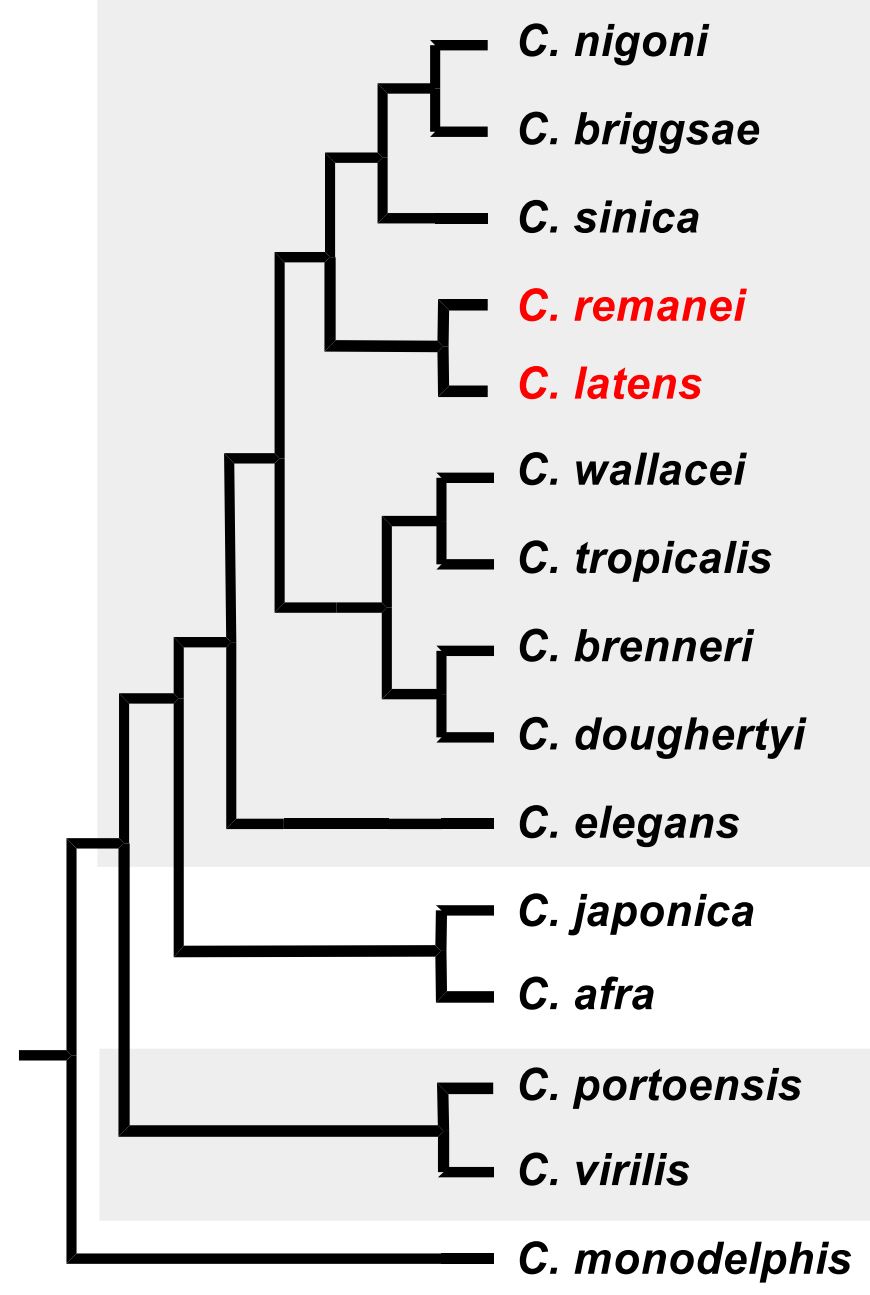


Figure S13: Sex-specific UHPLC-ESI-(-)-HRMS analysis of C. remanei PB4641 exometabolome extracts from male or female populations (150 worms each at 1 worm / $\mu \mathrm{l} \mathrm{S-medium} \mathrm{with} 1 \% E$. coli OP50 cultivated at $150 \mathrm{rpm}$ and $20^{\circ} \mathrm{C}$ for $12 \mathrm{~h}$ ) shows that asc-C4-cyC11 (fasc\#1, 3) is almost exclusively produced by females. Quantification was performed by comparison with an authentic standard.
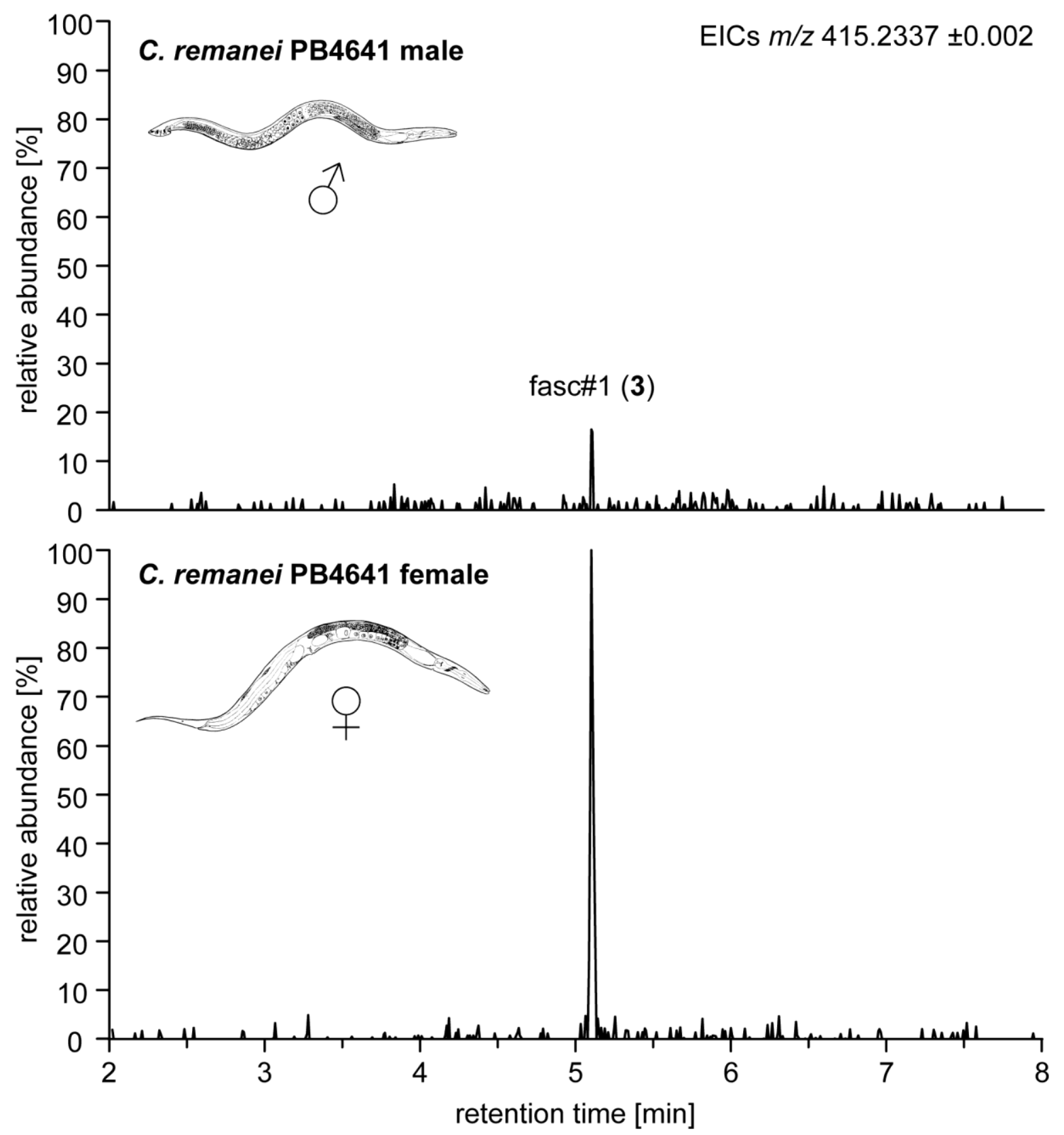
Figure S14: Holding assay shows suppression of $C$. remanei PB4641 female response to scoring regions conditioned with $100 \mathrm{fmol}$ asc-C4-cyC11 (fasc\#1, 3) upon pretreatment with $10 \mathrm{pmol}$ for approximately $20 \pm 5 \mathrm{~s}$ followed by 5 minutes recovery on ascaroside free plates $(\mathrm{n}=20$ each).
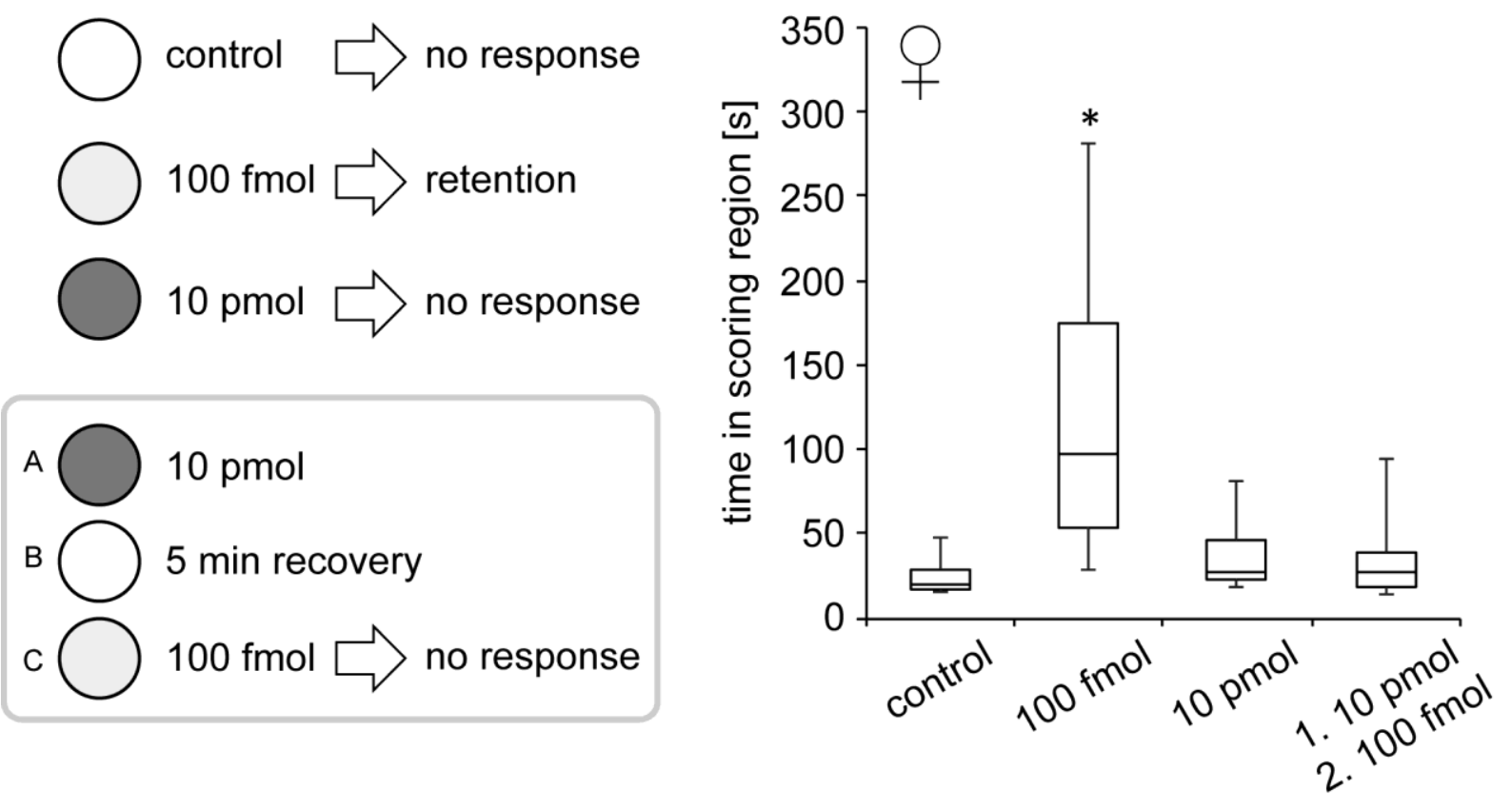
Figure S15: Holding assay representing mean times female (a) and male (b) C. remanei PB4641 spent in scoring regions conditioned with asc-C4-cyC11 (fasc\#1, 3) or the corresponding building blocks (3S,4S,7S)-7-hydroxy-cis-3,4-methylenedecanoic acid (8) and asc-C4 (ascr\#11, 19), individually or in combination at $1 \mathrm{fmol}$ and $1 \mathrm{pmol}$ shows that $C$. remanei retention depends on the intact fatty acid ascaroside structure $\left(n=20\right.$ each, $\left.{ }^{*} P<0.001\right)$.
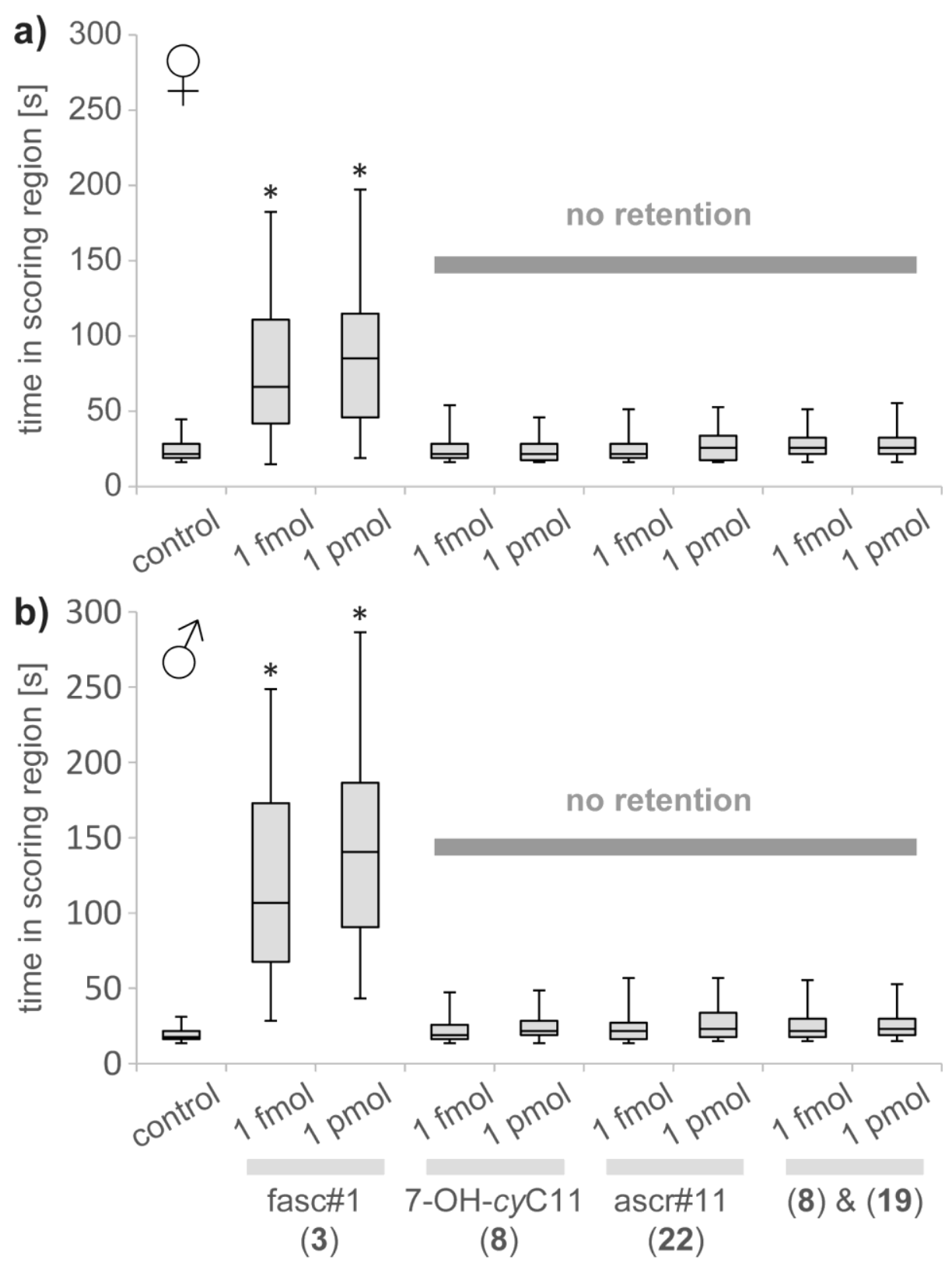
Figure S16a: Holding assay representing mean times female (a) and male (b) C. remanei PB4641 spent in scoring regions conditioned with isolated asc-C4-C12:1 (fasc\#2, 4) shows no retention ( $n=20$ each).
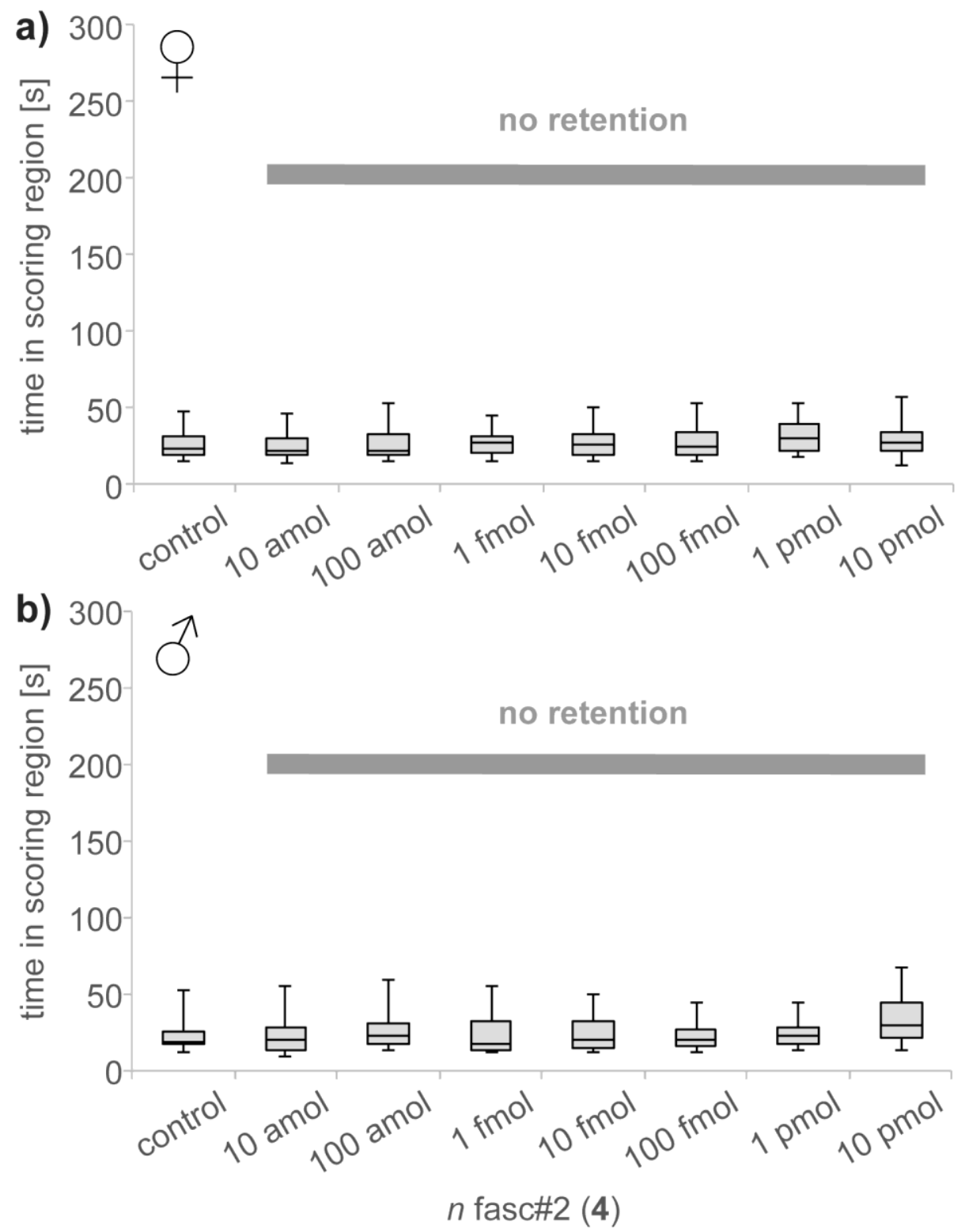
Figure S16b: Holding assay representing mean times female (a) and male (b) C. remanei PB4641 spent in scoring regions conditioned with enriched asc-C4-C10:1 (fasc\#3, 5) shows no retention ( $n=20$ each).
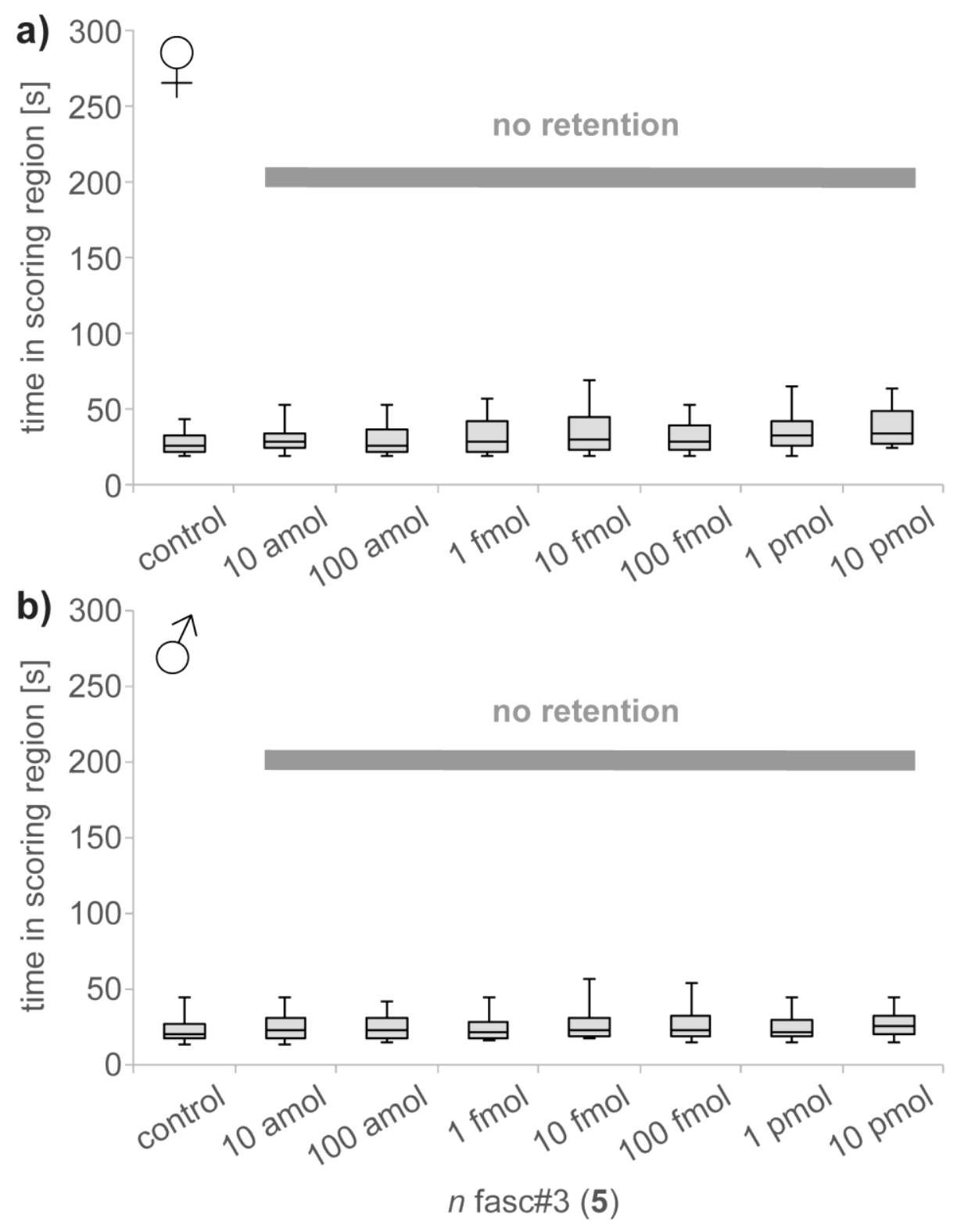
Figure S17: Comparative GC-EIMS analysis of FAMEs from the endolipidome of $C$. remanei PB4641 fed with (a) E. coli OP50, (b) E. coli $\Delta c f a$, and (c) $E$. coli $\Delta c f a$ supplemented with synthetic ( \pm -cis-9,10-methylenehexadecanoic acid $(c y C 17,25)$.
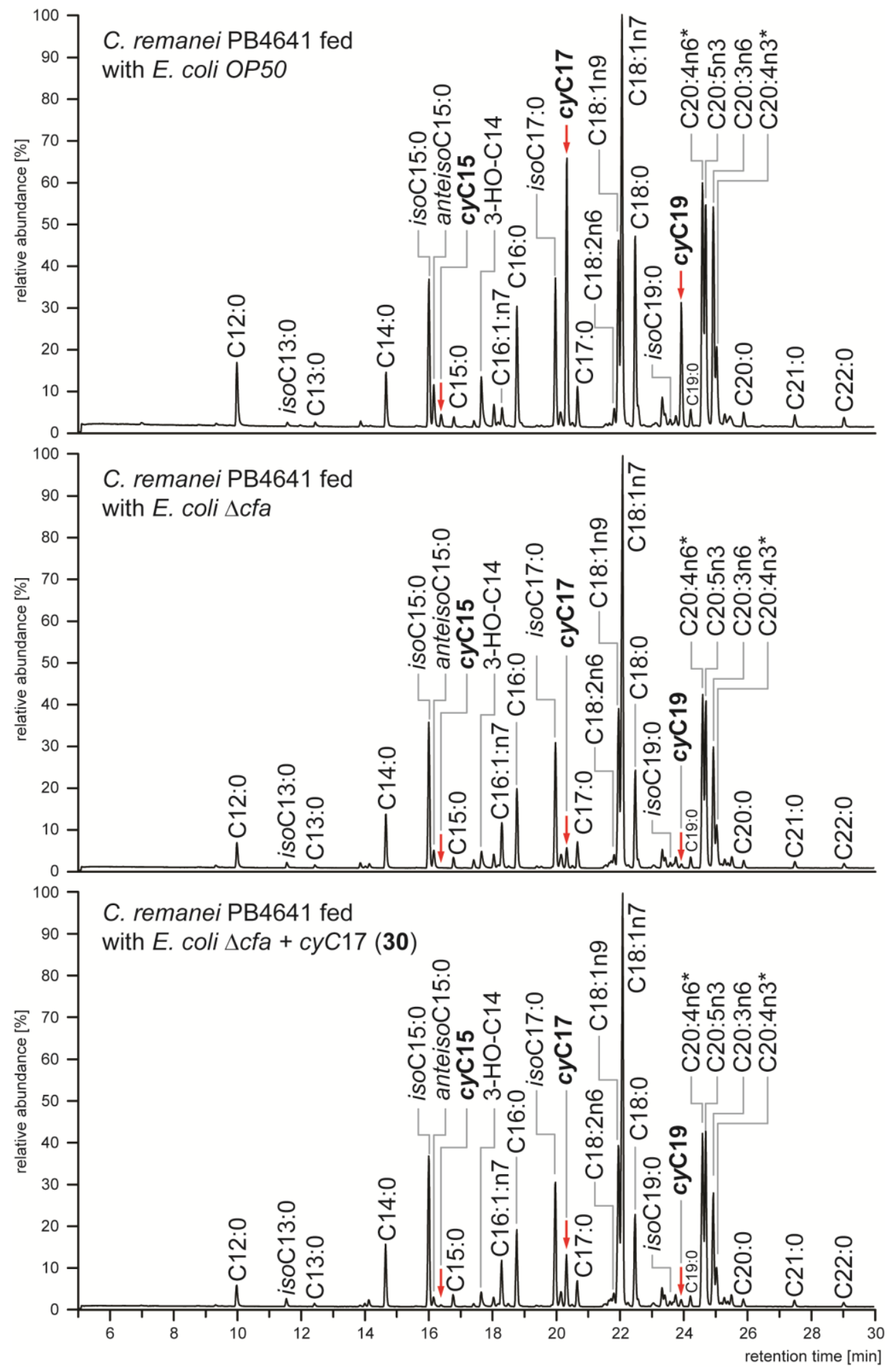
Figure S18: Comparative NMR endometabolome analysis of (a) E. coli OP50 and (b) the E. coli $\Delta c f a$ mutant shows lack of cyclopropyl fatty acids (cyC17, cyC19) and accumulation of their unsaturated precursors (C16:1n7, C18:1n7).

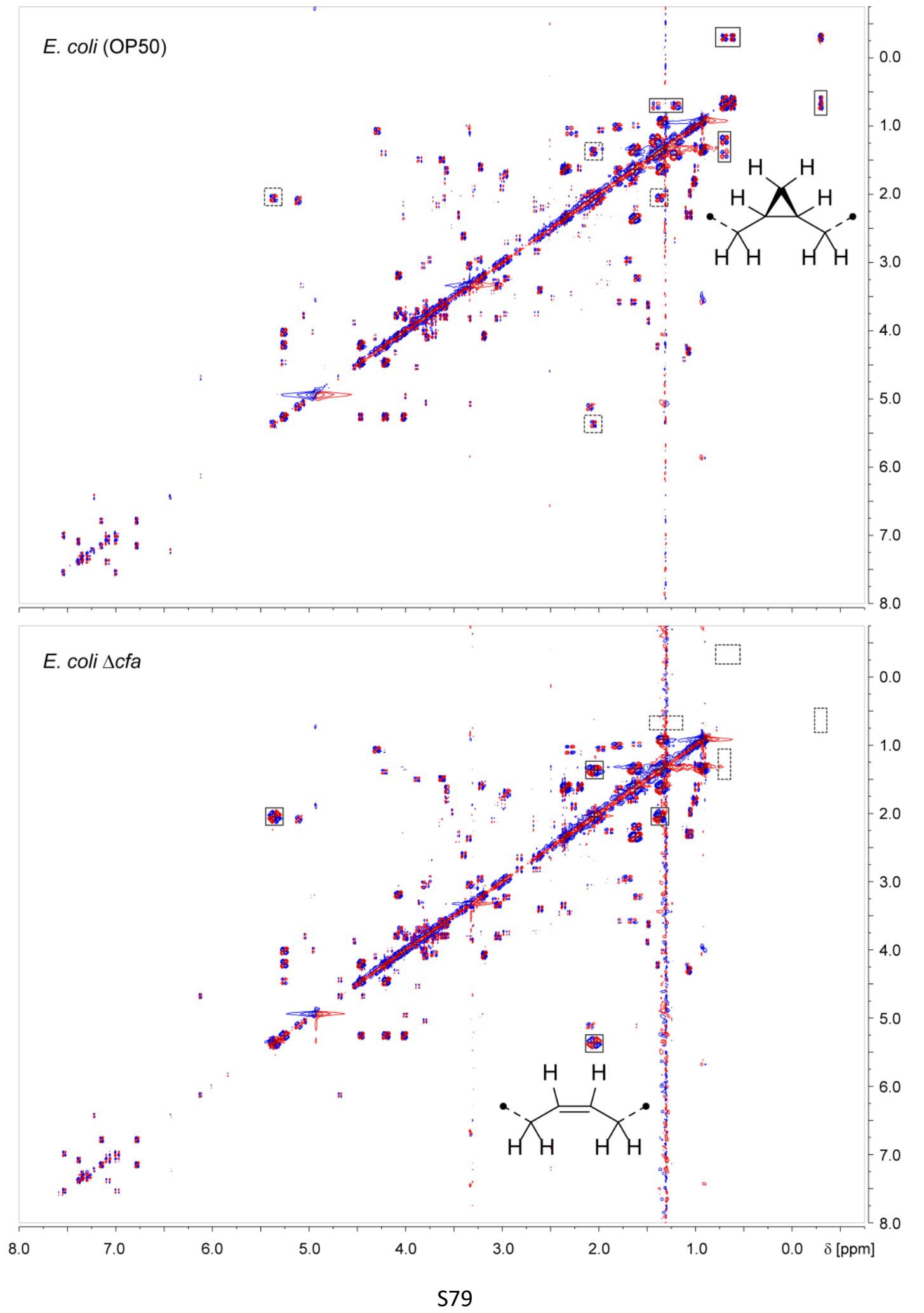


Figure S19: Comparative FAME analysis of (a) E. coli OP50 and (b) the E. coli $\Delta$ cfa mutant by GC-EIMS shows lack of cyclopropyl fatty acids cyC17 (25) and cy19 along with an accumulation of their unsaturated precursors palmitoleic acid (C16:1n7) and cis-vaccenic acid (C18:1n7).

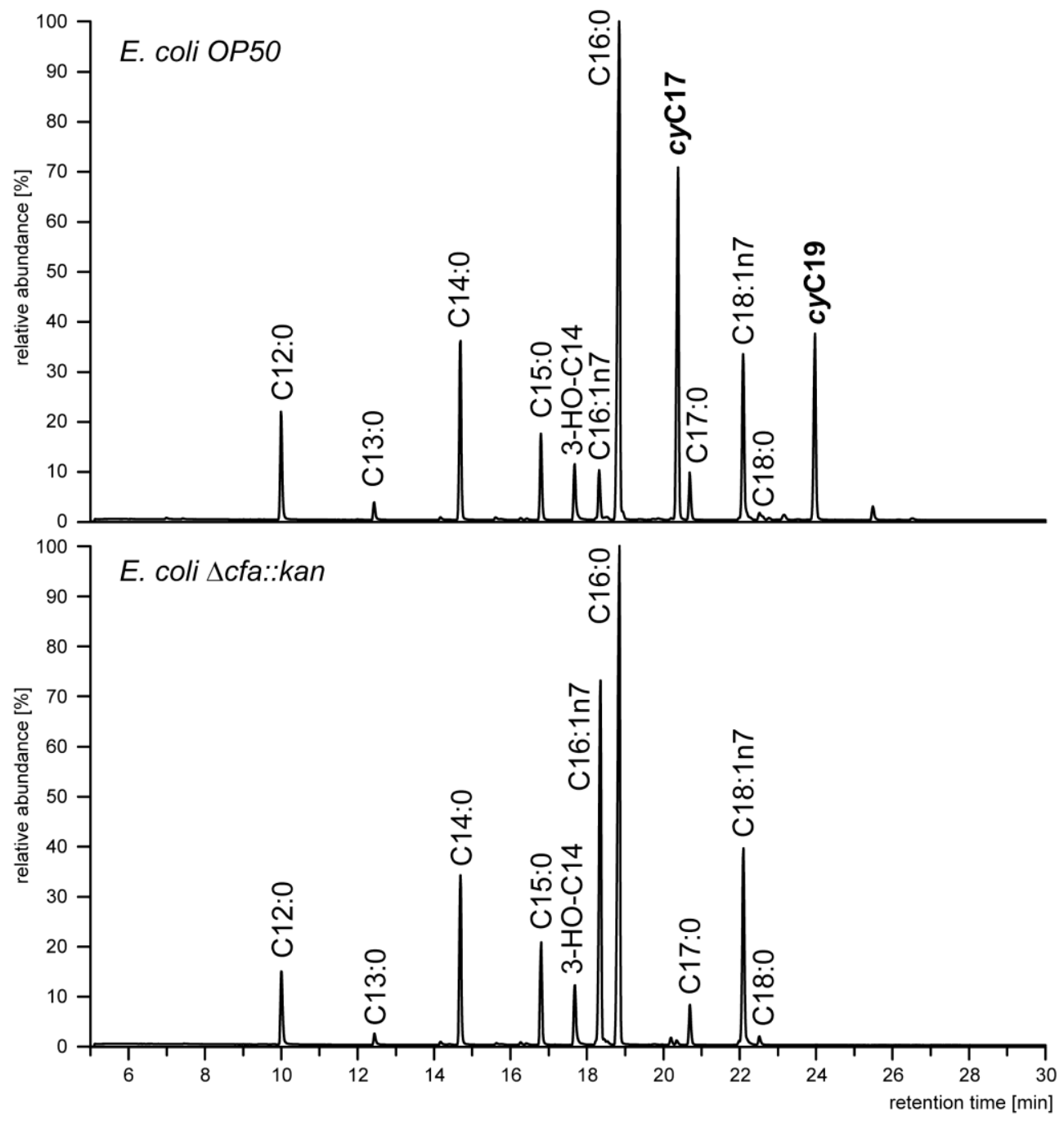


Figure S20: Bacterial cfa-dependent cyclopropyl fatty acids (cyC11 (26), cyC11DA (27), cyC9DA (28), cyC7DA (29)) from C. remanei PB4641 detected by differential analysis of cultures fed with $E$. coli OP50 and E. coli $\Delta$ cfa.

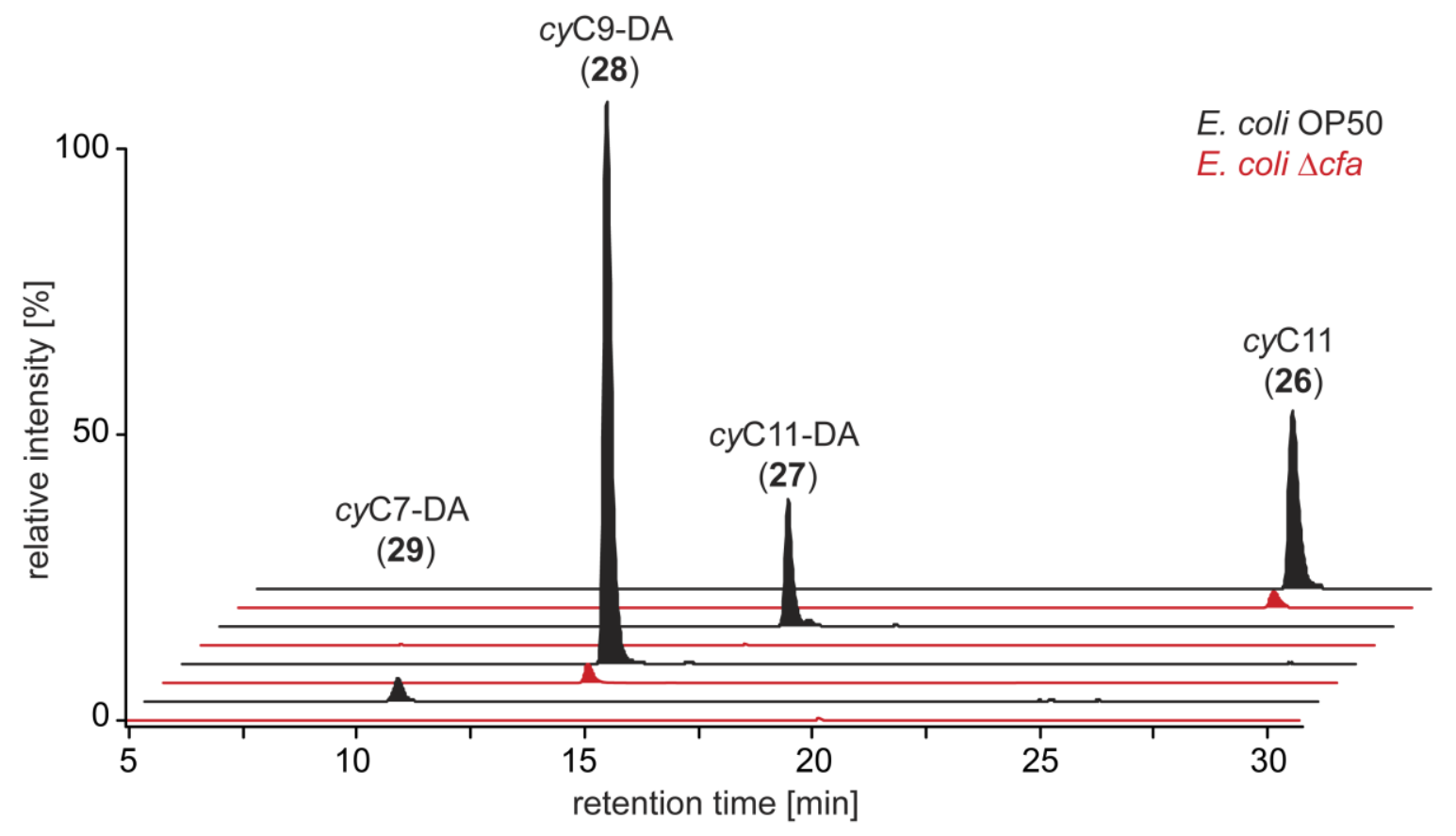


Figure S21: Identification of 7-hydroxy-cis-3,4-methylenedecanoic acid (8) in the isomeric 9hydroxy-cis-3,4-methylenedecanoic acid in Caenorhabditis species.
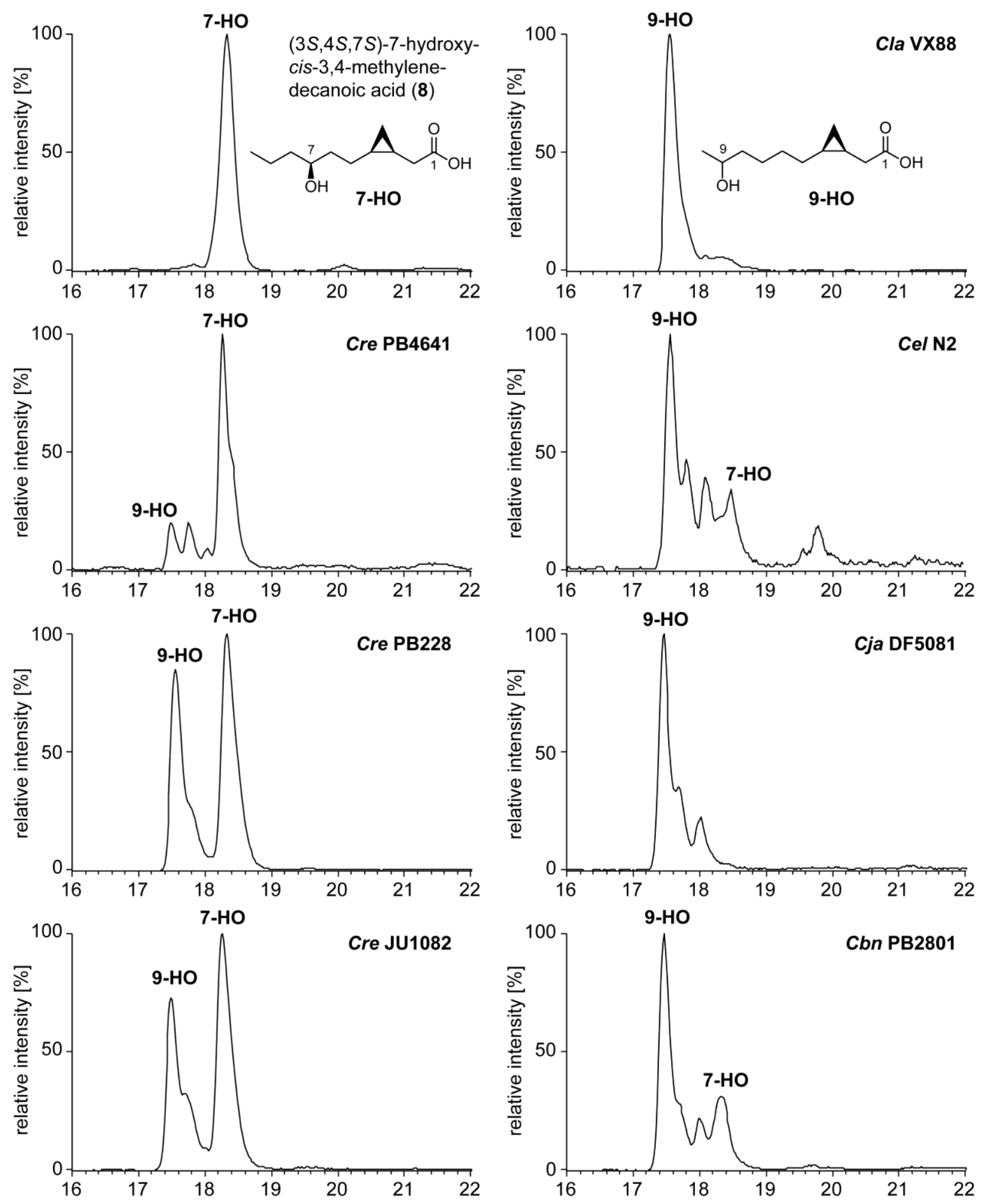


\section{Supplementary NMR Spectra}

Figure $\quad$ Page

S22 $\quad{ }^{1} \mathrm{H}$ NMR of natural asc-C4-cyC11 (fasc\#1, 3) from C. remanei. S88

S23 dqf-COSY of natural asc-C4-cyC11 (fasc\#1, 3) from C. remanei. $\quad$ S89

S24 ${ }^{1} \mathrm{H}$ NMR of natural asc-C4-cyC11 (fasc\#1, 3) from C. remanei. $\quad$ S90

S25 dqf-COSY of natural asc-C4-cyC11 (fasc\#1, 3) from C. remanei. S91

S26 HSQC of natural asc-C4-cyC11 (fasc\#1, 3) from C. remanei. $\quad$ S92

S27 HMBC of natural asc-C4-cyC11 (fasc\#1, 3) from C. remanei. $\quad$ S93

S28 NOESY of natural asc-C4-cyC11 (fasc\#1, 3) from C. remanei. $\quad$ S94

S29 presat-NOESY of natural asc-C4-cyC11 (fasc\#1, 3) from C. remanei. S95

S30 $\quad{ }^{1} \mathrm{H}$ NMR of natural asc-C4-C12:1 (fasc\#2, 4) from C. remanei. S96

S31 dqf-COSY of asc-C4-C12:1 (fasc\#2, 4) from C. remanei. S97

S32 ${ }^{1} \mathrm{H}$ NMR of natural asc-C4-C12:1 (fasc\#2, 4) from C. remanei. $\quad$ S98

S33 HSQC of natural asc-C4-C12:1 (fasc\#2, 4) from C. remanei. S99

S34 HMBC of natural asc-C4-C12:1 (fasc\#2, 4) from C. remanei. $\quad$ S100

S35 $\quad{ }^{1} \mathrm{H}$ NMR of natural asc-C4-C8 (fasc\#4, 7) from C. latens. S101

S36 ${ }^{1} \mathrm{H}$ NMR of natural asc-C4-C8 (fasc\#4, 7) from C. latens. S102

S37 dqf-COSY of natural asc-C4-C8 (fasc\#4, 7) from C. latens. S103

S38 HSQC of natural asc-C4-C8 (fasc\#4, 7) from C. latens. S104

S39 HMBC of natural asc-C4-C8 (fasc\#4, 7) from C. latens. S105

S40 $\quad{ }^{1} \mathrm{H}$ NMR of natural asc-C4-C6 (fasc\#5) from C. latens. $\quad$ S106

S41 dqf-COSY of natural asc-C4-C6 (fasc\#5) from C. latens. $\quad$ S107

S42 HSQC of natural asc-C4-C6 (fasc\#5) from C. latens. S108

S43 ${ }^{1} \mathrm{H}$ NMR of natural 7-hydroxy-3,4-cis-methylene decanoic acid (8). S109

S44 COSY of natural 7-hydroxy-3,4-cis-methylene decanoic acid (8). S110 
S45 ${ }^{1}$ H NMR of natural 10-hydroxy-3,4-cis-methylene decanoic acid.

S46 COSY of natural 10-hydroxy-3,4-cis-methylene decanoic acid.

S47 ${ }^{1} \mathrm{H}$ NMR of methyl 7-hydroxy-cis-3,4-metylene decanoate (9).

S48 dqf-COSY of methyl 7-hydroxy-cis-3,4-metylene decanoate (9).

S49 ${ }^{1} \mathrm{H}$ NMR of methyl 7-hydroxy-cis-3,4-metylene decanoate (9).

S50a SELTOCSY of methyl 7-hydroxy-cis-3,4-metylene decanoate (9).

S50b SELTOCSY of methyl 7-hydroxy-cis-3,4-metylene decanoate (9).

S50C SELTOCSY of methyl 7-hydroxy-cis-3,4-metylene decanoate (9).

S51 dqf-COSY of methyl 7-hydroxy-cis-3,4-metylene decanoate (9). S119

S52 HSQC of methyl 7-hydroxy-cis-3,4-metylene decanoate (9). $\quad$ S120

S53 $\quad{ }^{1} \mathrm{H}$ NMR of methyl 7-hydroxy-cis-3,4-methylene decanoate $(R)-\mathrm{MTPA}$ ester $((R)-10) . \quad$ S121

S54a SELTOCSY of methyl 7-hydroxy-cis-3,4-methylene decanoate $(R)$-MTPA ester $((R)-10) . \quad$ S122

S54b SELTOCSY of methyl 7-hydroxy-cis-3,4-methylene decanoate $(R)$-MTPA ester $((R)-10) . \quad$ S123

S54c SELTOCSY of methyl 7-hydroxy-cis-3,4-methylene decanoate $(R)$-MTPA ester $((R)-10) . \quad$ S124

S54d SELTOCSY of methyl 7-hydroxy-cis-3,4-methylene decanoate $(R)$-MTPA ester $((R)-10) . \quad$ S125

S55 dqf-COSY of methyl 7-hydroxy-cis-3,4-methylene decanoate $(R)$-MTPA ester $((R)-10) . \quad$ S126

S56 HSQC of methyl 7-hydroxy-cis-3,4-methylene decanoate (R)-MTPA ester ((R)-10). $\quad$ S127

S57 $\quad{ }^{1} \mathrm{H}$ NMR of methyl 7-hydroxy-cis-3,4-methylene decanoate (S)-MTPA ester ((S)-10). $\quad$ S128

S58a SELTOCSY of methyl 7-hydroxy-cis-3,4-methylene decanoate (S)-MTPA ester ((S)-10). $\quad$ S129

S58b SELTOCSY of methyl 7-hydroxy-cis-3,4-methylene decanoate (S)-MTPA ester ((S)-10). S130

S58c SELTOCSY of methyl 7-hydroxy-cis-3,4-methylene decanoate (S)-MTPA ester ((S)-10). $\quad$ S131

S58d SELTOCSY of methyl 7-hydroxy-cis-3,4-methylene decanoate (S)-MTPA ester ((S)-10). S132

S59 dqf-COSY of methyl 7-hydroxy-cis-3,4-methylene decanoate (S)-MTPA ester ((S)-10). S133

S60 HSQC of methyl 7-hydroxy-cis-3,4-methylene decanoate (S)-MTPA ester ((S)-10). S134

S61 ${ }^{1} \mathrm{H}$ NMR of 5-O-tert-butyldimethylsilyloxy-pent-2-yn-1-ol. $\quad$ S135

S62 $\quad{ }^{13} \mathrm{C}$ NMR of 5-O-tert-butyldimethylsilyloxy-pent-2-yn-1-ol. $\quad$ S136 
S63 ${ }^{1} \mathrm{H}$ NMR of (Z)-5-O-tert-butyldimethylsilyloxy-pent-2-en-1-ol (11).

S64 $\quad{ }^{13} \mathrm{C}$ NMR of (Z)-5-O-tert-butyldimethylsilyloxy-pent-2-en-1-ol (11).

S65 ${ }^{1} \mathrm{H}$ NMR of 5-O-tert-butyldimethylsilyloxy-cis-2,3-methylenepentan-1-ol (12).

S66 ${ }^{13} \mathrm{C}$ NMR of 5-O-tert-butyldimethylsilyloxy-cis-2,3-methylenepentan-1-ol (12).

S67 dqf-COSY of 5-O-tert-butyldimethylsilyloxy-cis-2,3-methylenepentan-1-ol (12).

S68 ${ }^{1} \mathrm{H}$ NMR of 5-O-tert-butyldimethylsilyloxy-cis-2,3-methylenepentanal (13).

S69 $\quad{ }^{13} \mathrm{C}$ NMR of 5-O-tert-butyldimethylsilyloxy-cis-2,3-methylenepentanal (13).

S70 dqf-COSY of 5-O-tert-butyldimethylsilyloxy-cis-2,3-methylenepentanal (13).

S71 HSQC of 5-O-tert-butyldimethylsilyloxy-cis-2,3-methylenepentanal (13).

S72 ${ }^{1} \mathrm{H}$ NMR of 6-O-tert-butyldimethylsilyloxy-cis-3,4-methylene-1-hexene (14). S146

S73 ${ }^{13} \mathrm{C}$ NMR of 6-O-tert-butyldimethylsilyloxy-cis-3,4-methylene-1-hexene (14). S147

S74 ${ }^{1} \mathrm{H}$ NMR of 1-hexen-3-yl acetate (15). S148

S75 ${ }^{13} \mathrm{C}$ NMR of 1-hexen-3-yl acetate (15). S149

S76 ${ }^{1} \mathrm{H}$ NMR of $(E)-1-O-t e r t-b u t y l d i m e t h y l s i l y l o x y-c i s-3,4-m e t h y l e n e d e c-5-e n-7-y l$ acetate (16). S150

S77 $\quad{ }^{13} \mathrm{C}$ NMR of $(E)-1-O-t e r t-b u t y l d i m e t h y l s i l y l o x y-c i s-3,4-m e t h y l e n e d e c-5-e n-7-y l$ acetate (16). S151

S78 dqf-COSY of (E)-1-O-tert-butyldimethylsilyloxy-cis-3,4-methylenedec-5-en-7-yl acetate (16). S152

S79 ${ }^{1} \mathrm{H}$ NMR of 1-O-tert-butyldimethylsilyloxy-cis-3,4-methylenedecan-7-yl acetate (17). S153

S80 $\quad{ }^{13} \mathrm{C}$ NMR of 1-O-tert-butyldimethylsilyloxy-cis-3,4-methylenedecan-7-yl acetate (17). S154

S81 dqf-COSY of 1-O-tert-butyldimethylsilyloxy-cis-3,4-methylenedecan-7-yl acetate (17). S155

S82 HSQC of 1-O-tert-butyldimethylsilyloxy-cis-3,4-methylenedecan-7-yl acetate (17). S156

S83 ${ }^{1} \mathrm{H}$ NMR of 1-O-tert-butyldimethylsilyloxy-cis-3,4-methylenedecan-7-ol (18). S157

S84 ${ }^{1} \mathrm{H}$ NMR of (3R)-methyl 3-[(3,6-dideoxy-a-L-arabino-hexopyranosyl)oxy]-butanoate. S158

S85 $\quad{ }^{13} \mathrm{C}$ NMR of (3R)-methyl 3-[(3,6-dideoxy-a-L-arabino-hexopyranosyl)oxy]-butanoate. S159

S86 ${ }^{1} \mathrm{H}$ NMR of (3R)-methyl 3-[(2,4-di-O-tert-butyldimethylsilyloxy-3,6-dideoxy-a-L-arabino- $\quad$ S160 hexopyranosyl)oxy]-butanoate.

S87 $\quad{ }^{13} \mathrm{C}$ NMR of (3R)-methyl 3-[(2,4-di-O-tert-butyldimethylsilyloxy-3,6-dideoxy-a-L-arabinohexopyranosyl)oxy]-butanoate. 
S88 dqf-COSY of (3R)-methyl 3-[(2,4-di-O-tert-butyldimethylsilyloxy-3,6-dideoxy-a-L-arabinohexopyranosyl)oxy]-butanoate.

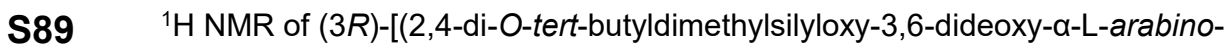
hexopyranosyl)oxy]-butanoic acid (20).

S90 ${ }^{1} \mathrm{H}$ NMR of 7-[(3'-[(2,4-di-O-tert-butyldimethylsilyloxy-3",6"-dideoxy-a-L-arabinohexopyranosyl)-oxy]-butanoyl)oxy]-3,4-cis-methylene-1-O-tert-butyldimethylsilyloxy decane (21)

S91 ${ }^{1} \mathrm{H}$ NMR of 7-[(3'-[(2,4-di-O-tert-butyldimethylsilyloxy-3",6"-dideoxy- $\alpha$-L-arabinohexopyranosyl)-oxy]-butanoyl)oxy]-3,4-cis-methylene-1-O-tert-butyldimethylsilyloxy decane (21).

S92 ${ }^{13} \mathrm{C}$ NMR of 7-[(3'-[(2,4-di-O-tert-butyldimethylsilyloxy-3",6"-dideoxy-a-L-arabinohexopyranosyl)-oxy]-butanoyl)oxy]-3,4-cis-methylene-1-O-tert-butyldimethylsilyloxy decane (21).

S93 dqf-COSY of 7-[(3'-[(2,4-di-O-tert-butyldimethylsilyloxy-3",6"-dideoxy-a-L-arabinohexopyranosyl)-oxy]-butanoyl)oxy]-3,4-cis-methylene-1-O-tert-butyldimethylsilyloxy decane (21).

S94 HSQC of 7-[(3'-[(2,4-di-O-tert-butyldimethylsilyloxy-3",6"-dideoxy- $\alpha$-L-arabinohexopyranosyl)-oxy]-butanoyl)oxy]-3,4-cis-methylene-1-O-tert-butyldimethylsilyloxy decane (21).

S95 ${ }^{1} \mathrm{H}$ NMR of 7-[(3'-[(3",6"-dideoxy-a-L-arabino-hexopyranosyl)-oxy]-butanoyl)oxy]-3,4-cismethylene-1-decanol (22).

S96 dqf-COSY of 7-[(3'-[(3",6"-dideoxy-a-L-arabino-hexopyranosyl)-oxy]-butanoyl)oxy]-3,4-cismethylene-1-decanol (22).

S97 HSQC of 7-[(3'-[(3",6"-dideoxy- $\alpha$-L-arabino-hexopyranosyl)-oxy]-butanoyl)oxy]-3,4-cismethylene-1-decanol (22).

S98 ${ }^{1} \mathrm{H}$ NMR of synthetic 7-[(3'-[(3",6"'-dideoxy-a-L-arabino-hexopyranosyl)-oxy]-butanoyl)oxy]3,4-cis-methylene decanoic acid (fasc\#1, 3).

S99 dqf-COSY of synthetic 7-[(3'-[(3",6"-dideoxy-a-L-arabino-hexopyranosyl)-oxy]-butanoyl)oxy]3,4-cis-methylene decanoic acid (fasc\#1, 3).

S100 HSQC of synthetic 7-[(3'-[(3",6"'-dideoxy-a-L-arabino-hexopyranosyl)-oxy]-butanoyl)oxy]-3,4cis-methylene decanoic acid (fasc\#1, 3).

S101 HMBC of synthetic 7-[(3'-[(3",6"'-dideoxy-a-L-arabino-hexopyranosyl)-oxy]-butanoyl)oxy]-3,4cis-methylene decanoic acid (fasc\#1, 3).

S102 ${ }^{1} \mathrm{H}$ NMR of synthetic 7-[(3'-[(3",6"-dideoxy-a-L-arabino-hexopyranosyl)-oxy]-butanoyl)oxy]3,4-cis-methylene decanoic acid (fasc\#1, 3).

S103 ${ }^{1} \mathrm{H}$ NMR of natural 7-[(3'-[(3",6"-dideoxy-a-L-arabino-hexopyranosyl)-oxy]-butanoyl)oxy]-3,4cis-methylene decanoic acid (fasc\#1, 3) from C. remanei PB4641. 
S105 ${ }^{13} \mathrm{C}$ NMR of methyl cis-9-hexadecenoate. $\quad$ S179

S106 ${ }^{1} \mathrm{H}$ NMR of $( \pm)$-methyl cis-9,10-metylenehexadecanoate. $\quad$ S180

S107 ${ }^{13} \mathrm{C}$ NMR of $( \pm)$-methyl cis-9,10-metylenehexadecanoate. $\quad$ S181

S108 ${ }^{1} \mathrm{H}$ NMR of $( \pm)$-cis-9,10-metylenehexadecanoic acid $(\mathbf{2 5}$, cyC17). S182

S109 ${ }^{13} \mathrm{C}$ NMR of $( \pm)$-cis-9,10-metylenehexadecanoic acid $(25$, cyC17). S183

S110 ${ }^{1} \mathrm{H}$ NMR of natural cis-3,4-methylene decanoic acid $(26, c y C 11) . \quad S 184$

S111 dqf-COSY of natural cis-3,4-methylene decanoic acid (26, cyC11). S185

S112 HSQC of natural cis-3,4-methylene decanoic acid (26, cyC11). S186

S113 ${ }^{1} \mathrm{H}$ NMR of cis-3,4-methylene decanedioic acid (27, cyC11DA). S187

S114 dqf-COSY of natural cis-3,4-methylene decanedioic acid (27, cyC11DA). S188

S115 ${ }^{1} \mathrm{H}$ NMR of natural cis-3,4-methylene octanedioic acid (28, cyC9DA). S189

S116 ${ }^{1} \mathrm{H}$ NMR of natural cis-3,4-methylene octanedioic acid (28, cyC9DA). $\quad$ S190

S117 ${ }^{13}$ C NMR of natural cis-3,4-methylene octanedioic acid (28, cyC9DA). $\quad$ S191

S118 dqf-COSY of natural cis-3,4-methylene octanedioic acid (28, cyC9DA). S192

S119 HSQC of natural cis-3,4-methylene octanedioic acid (28, cyC9DA). S193

S120 dqf-COSY of natural cis-3,4-methylene hexanedioic acid (29, cyC7DA). S194

S121 ${ }^{1} \mathrm{H}$ NMR of $E$. coli OP50 endometabolome extract. $\quad$ S195

S122 ${ }^{1} \mathrm{H}$ NMR sof $E$. coli $\Delta c f a$ endometabolome extract. $\quad$ S196

S123 dqf-COSY of E. coli OP50 endometabolome extract. $\quad$ S197

$\mathbf{S 1 2 4} d q$-COSY of $E$. coli $\Delta c f a$ endometabolome extract. $\quad S 198$ 
Figure S22: ${ }^{1} \mathrm{H}$ NMR $\left(400 \mathrm{MHz}, \mathrm{CD}_{3} \mathrm{OD}\right)$ of natural asc-C4-cyC11 (fasc\#1, 3) from C. remanei.

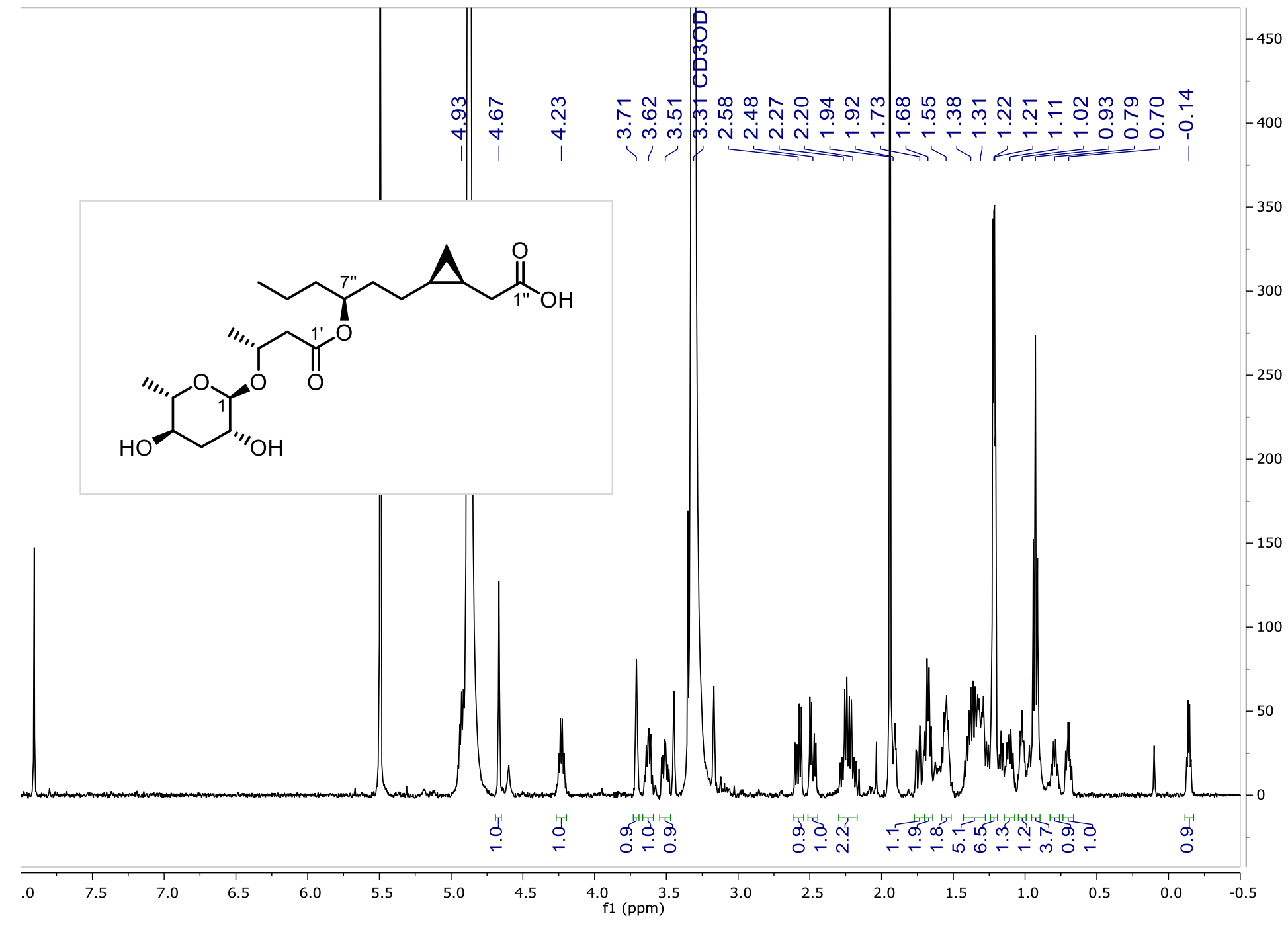


Figure S23: dqf-COSY (400 MHz, $\left.\mathrm{CD}_{3} \mathrm{OD}\right)$ of natural asc-C4-cyC11 (fasc\#1, 3) from C. remanei.

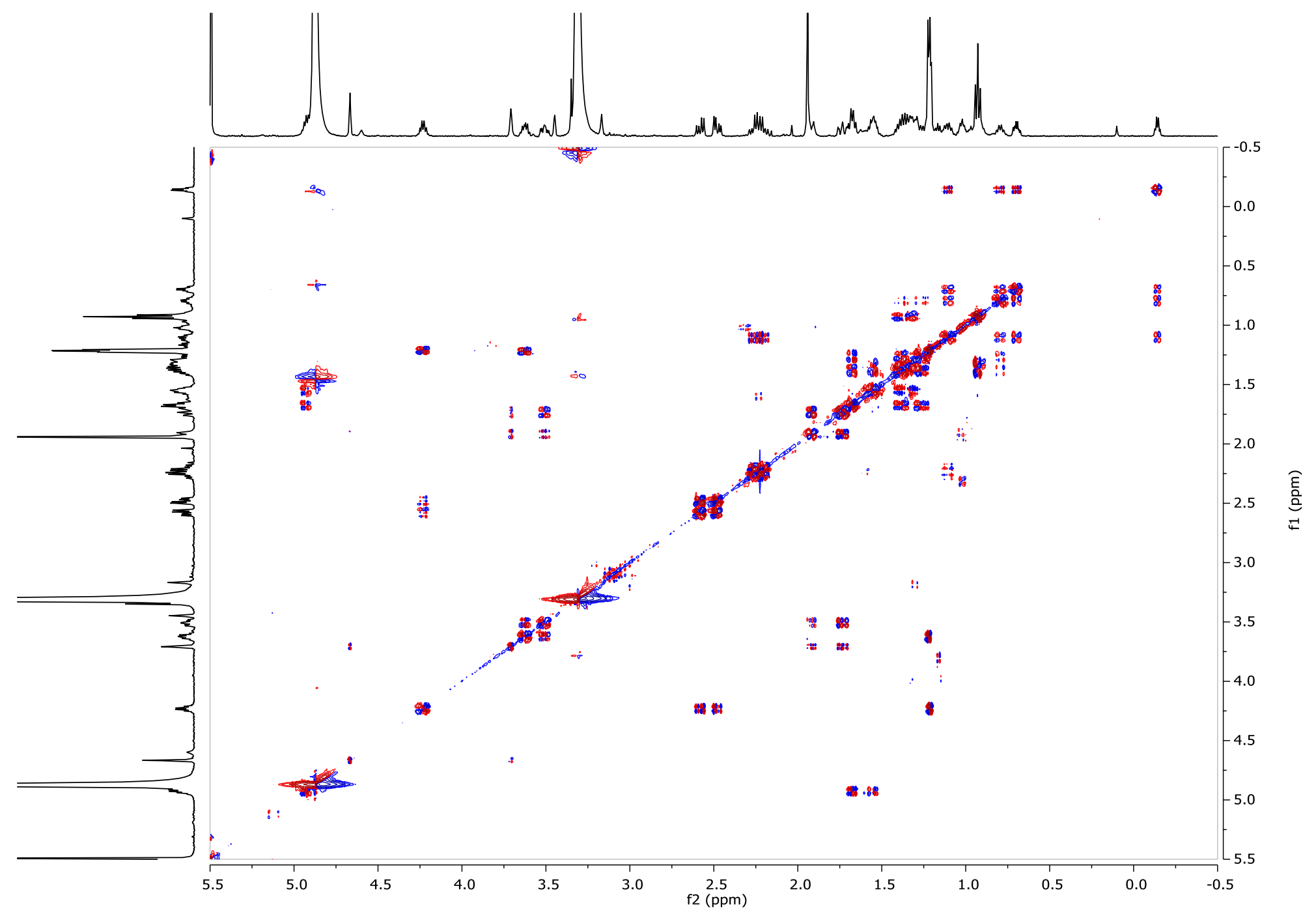


Figure S24: ${ }^{1} \mathrm{H}$ NMR $\left(700 \mathrm{MHz}, \mathrm{CD}_{3} \mathrm{OD}\right)$ of natural asc-C4-cyC11 (fasc\#1, 3) from C. remanei.

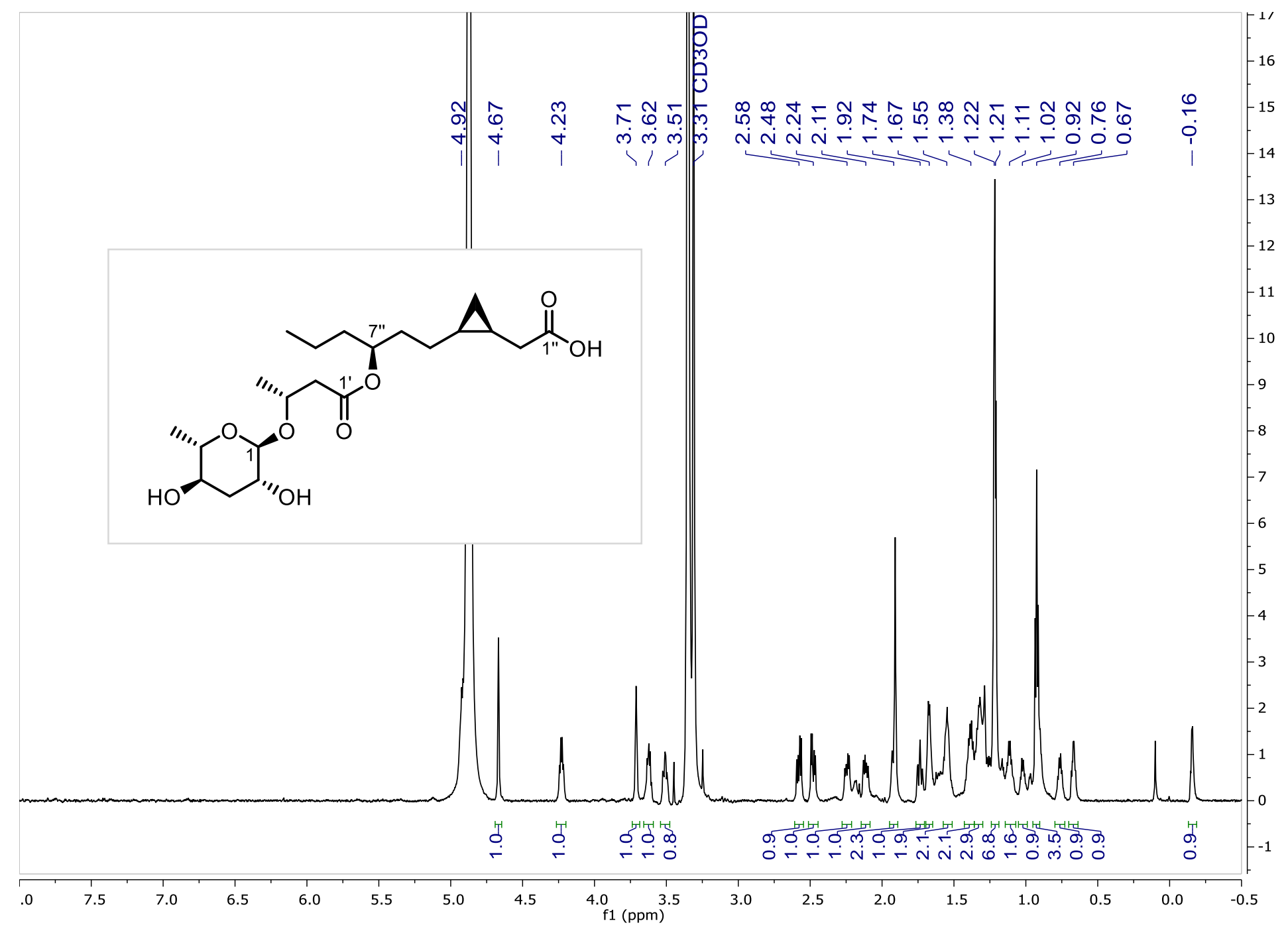


Figure S25: dqf-COSY (700 MHz, $\left.\mathrm{CD}_{3} \mathrm{OD}\right)$ of natural asc-C4-cyC11 (fasc\#1, 3) from C. remanei.

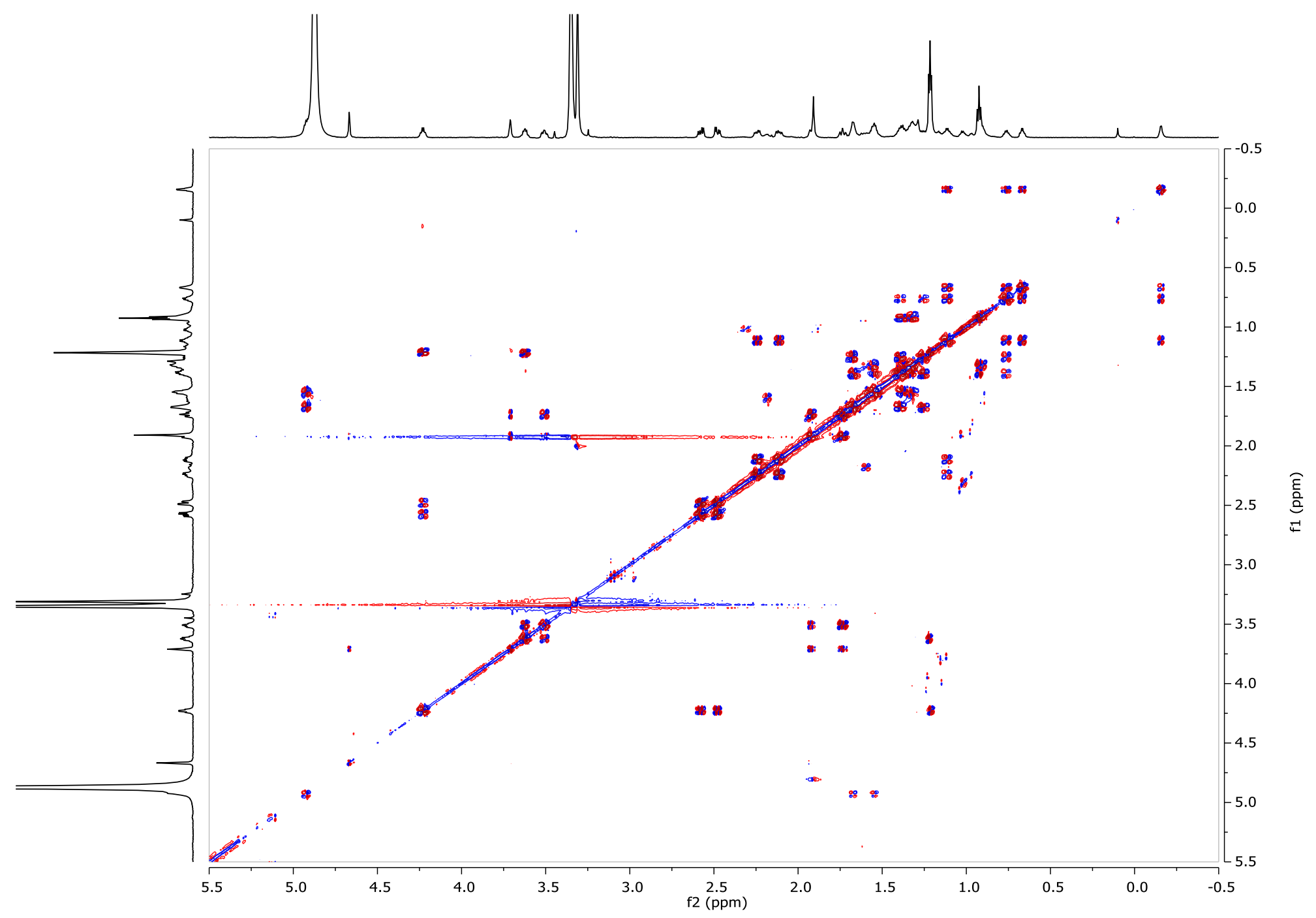


Figure S26: HSQC (700 MHz, $\left.\mathrm{CD}_{3} \mathrm{OD}\right)$ of natural asc-C4-cyC11 (fasc\#1, 3) from C. remanei.

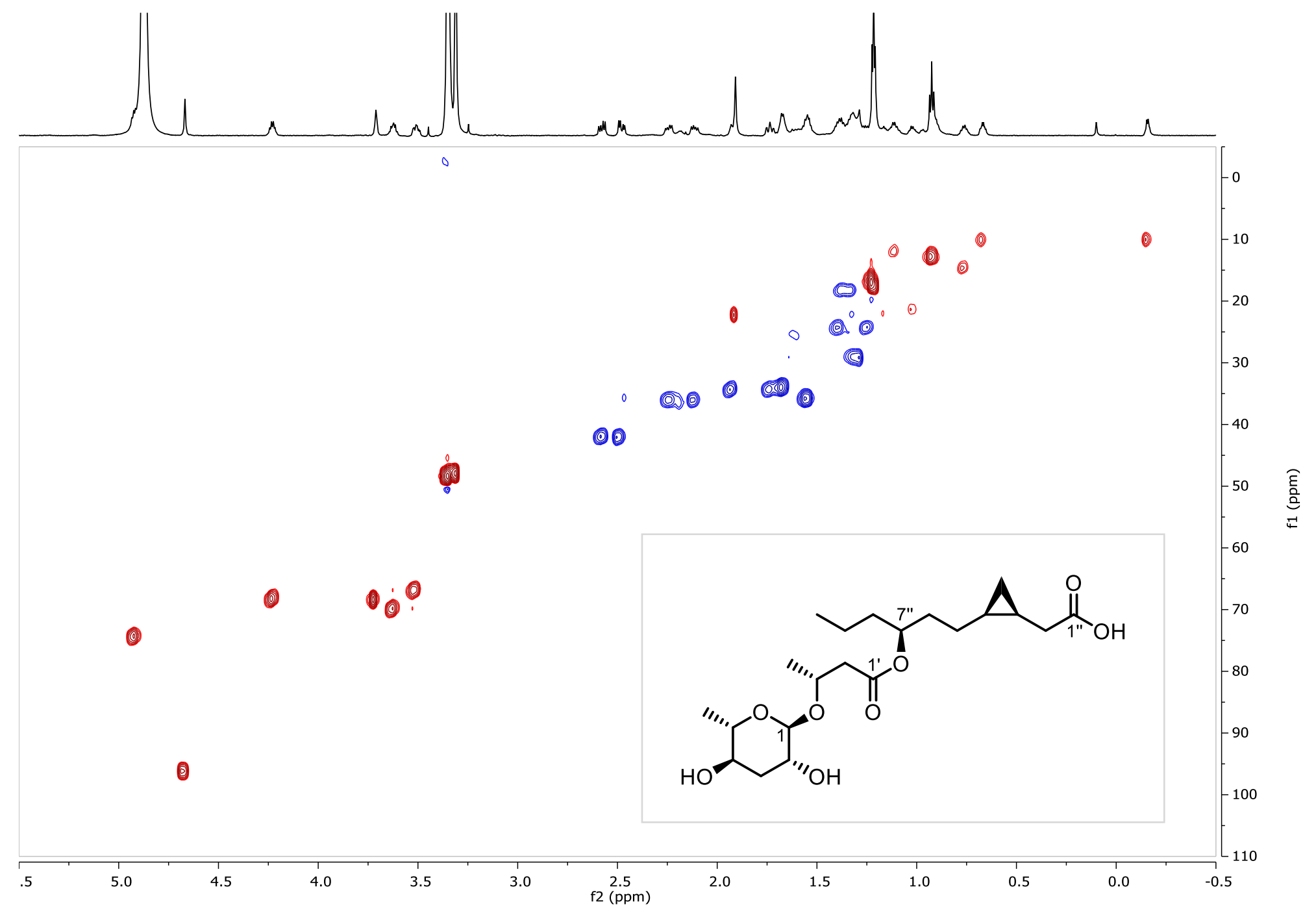


Figure S27: $\mathrm{HMBC}\left(700 \mathrm{MHz}, \mathrm{CD}_{3} \mathrm{OD}\right)$ of natural asc-C4-cyC11 (fasc\#1, 3) from C. remanei.

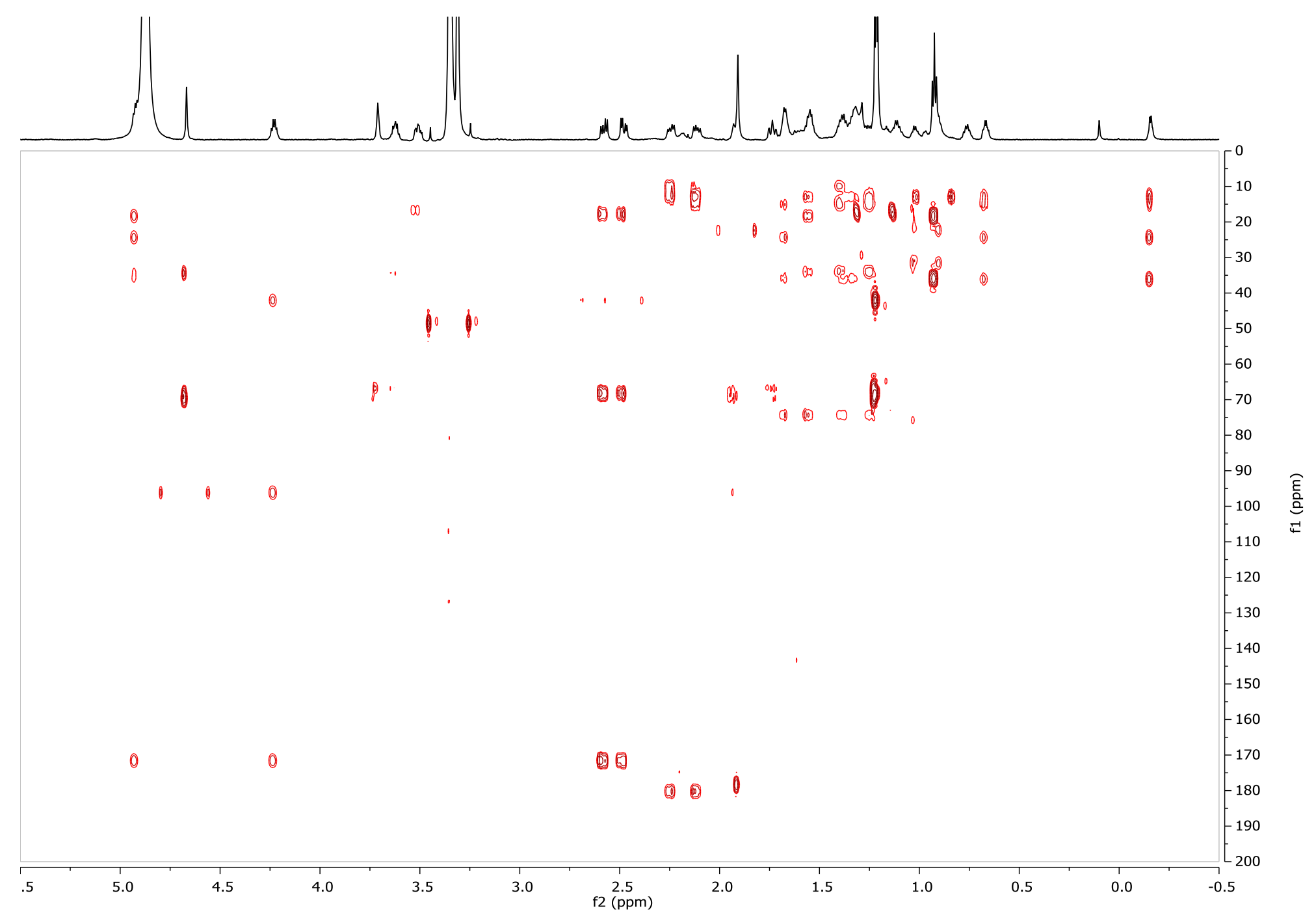


Figure S28: NOESY (700 MHz, CD $\left.{ }_{3} \mathrm{OD}\right)$ of natural asc-C4-cyC11 (fasc\#1, 3) from C. remanei.

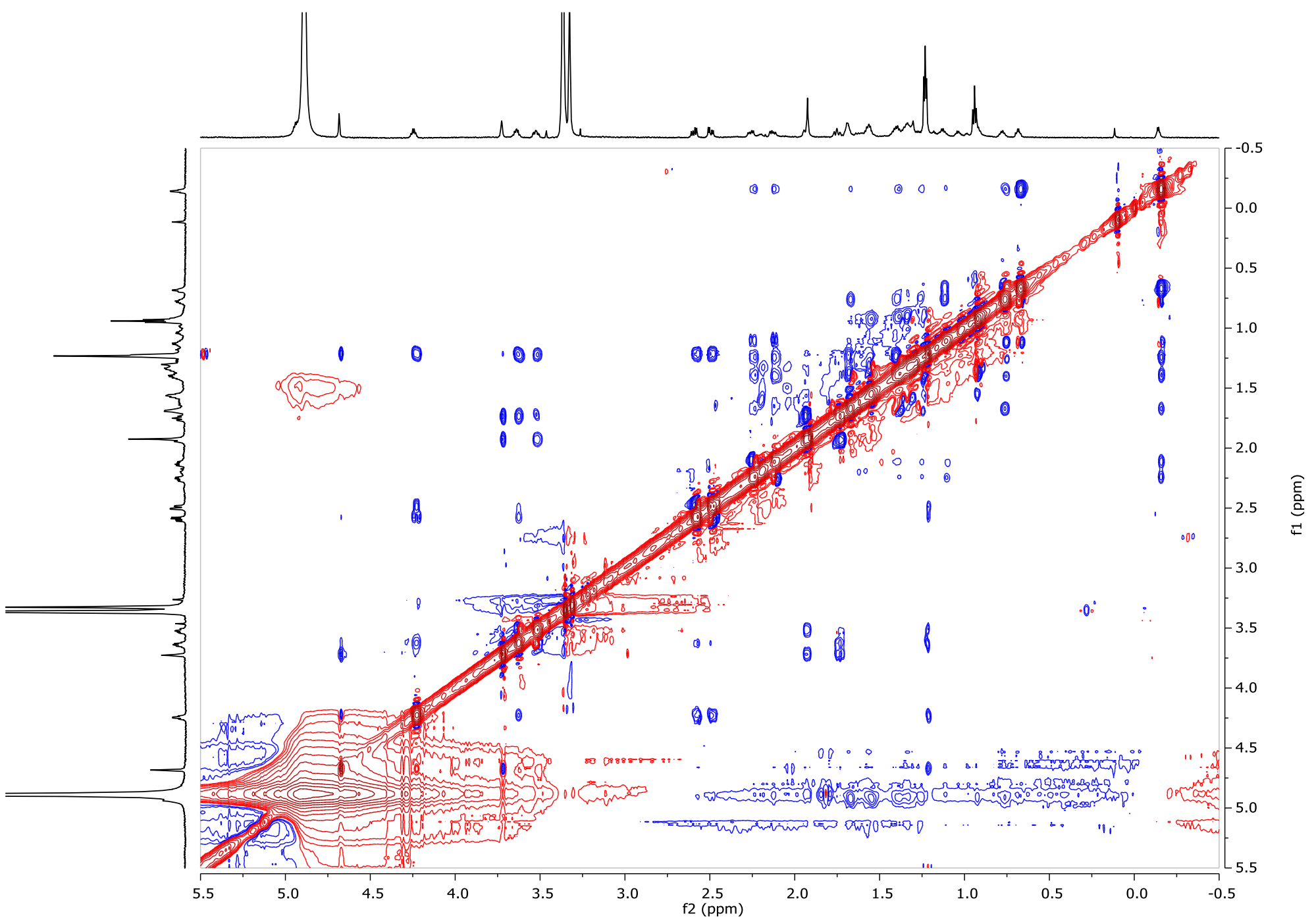


Figure S29: presat-NOESY $\left(700 \mathrm{MHz}, \mathrm{CD}_{3} \mathrm{OD}\right.$ ) of natural asc-C4-cyC11 (fasc\#1, 3) from C. remanei.

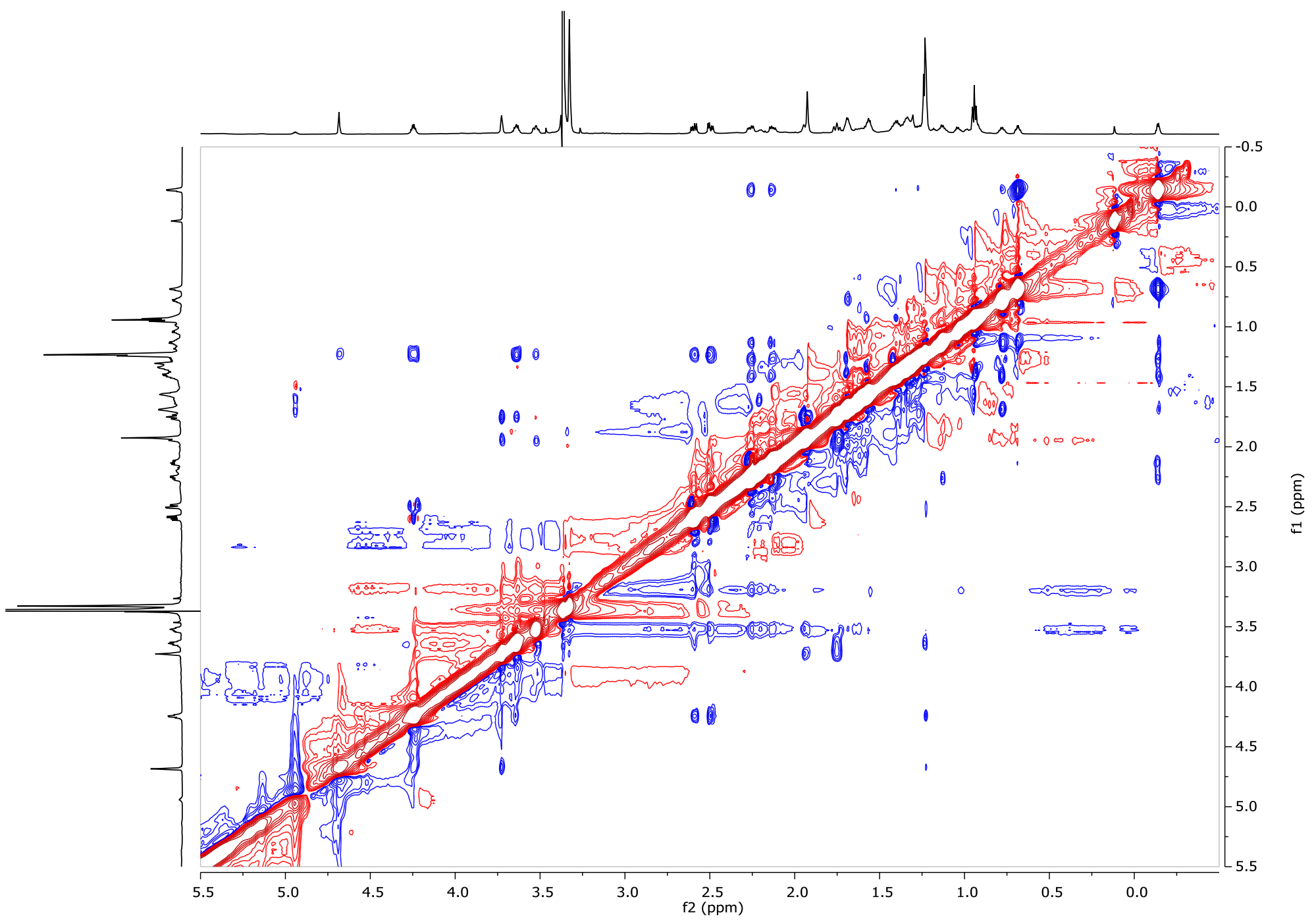


Figure S30: ${ }^{1} \mathrm{H}$ NMR (400 MHz, $\mathrm{CD}_{3} \mathrm{OD}$ ) of natural asc-C4-C12:1 (fasc\#2, 4) from C. remanei.

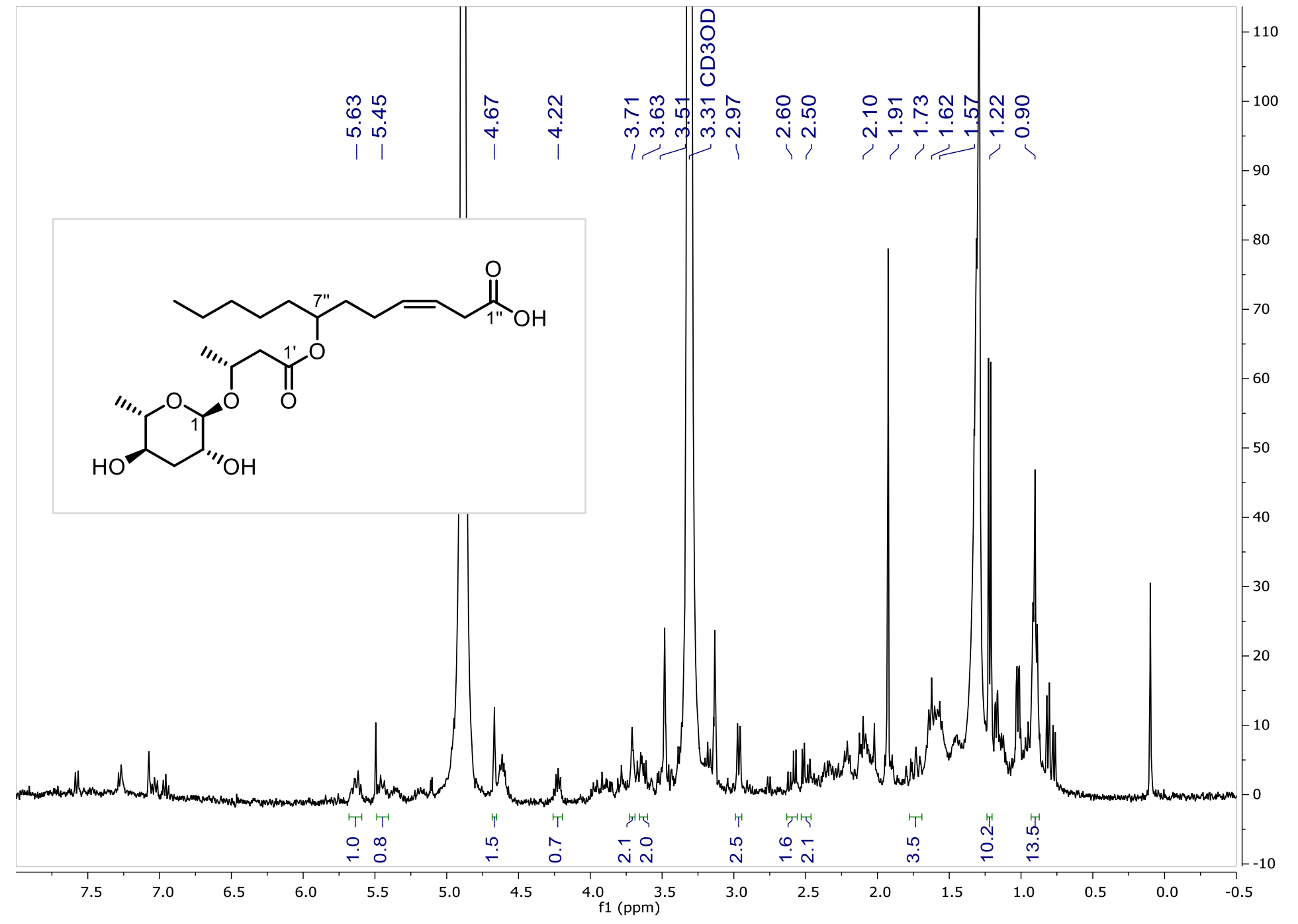


Figure S31: dqf-COSY (400 MHz, $\mathrm{CD}_{3} \mathrm{OD}$ ) of natural asc-C4-C12:1 (fasc\#2, 4) from C. remanei.

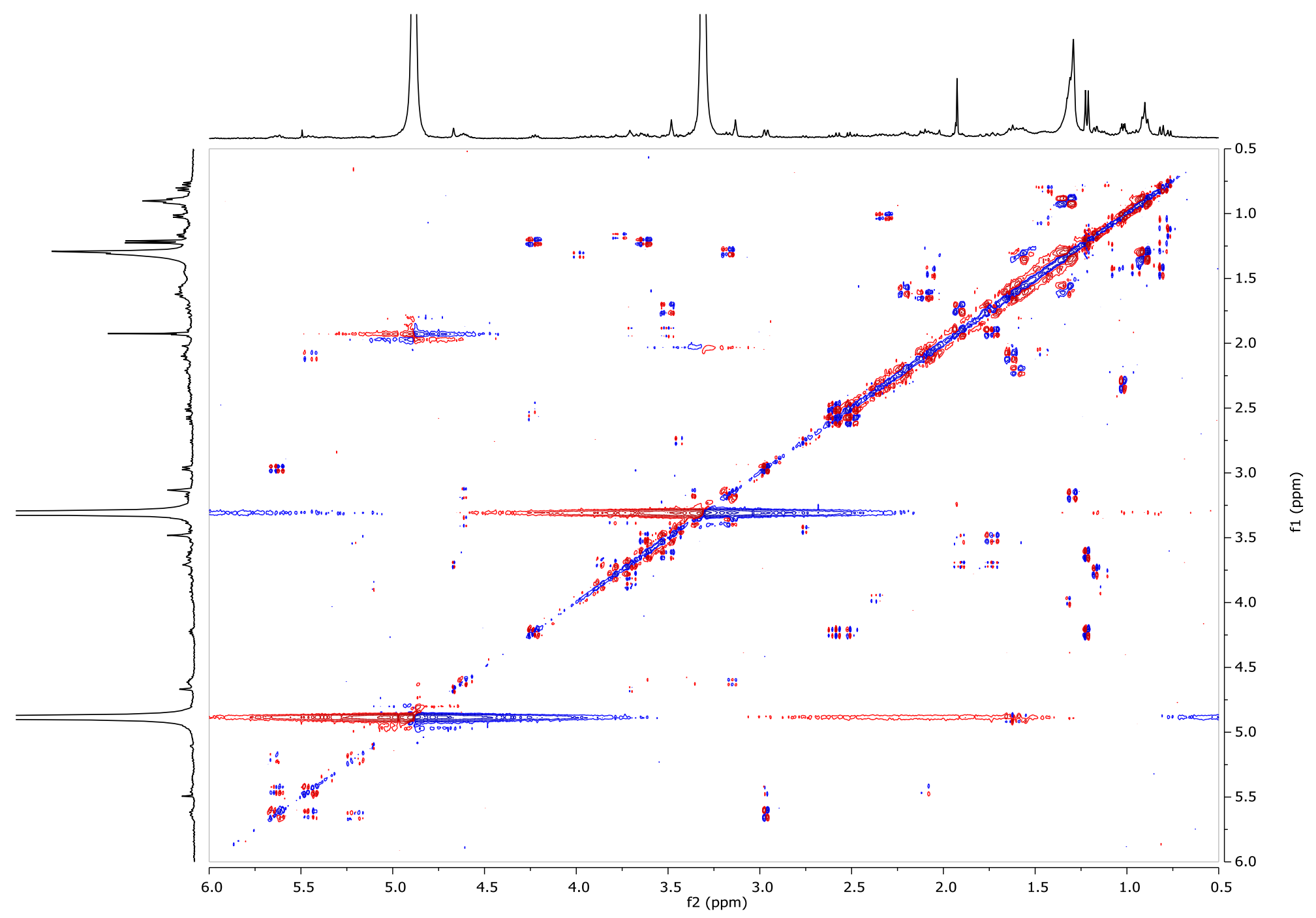


Figure S32: ${ }^{1} \mathrm{H}$ NMR $\left(700 \mathrm{MHz}, \mathrm{CD}_{3} \mathrm{OD}\right)$ of natural asc-C4-C12:1 (fasc\#2, 4) from C. remanei.

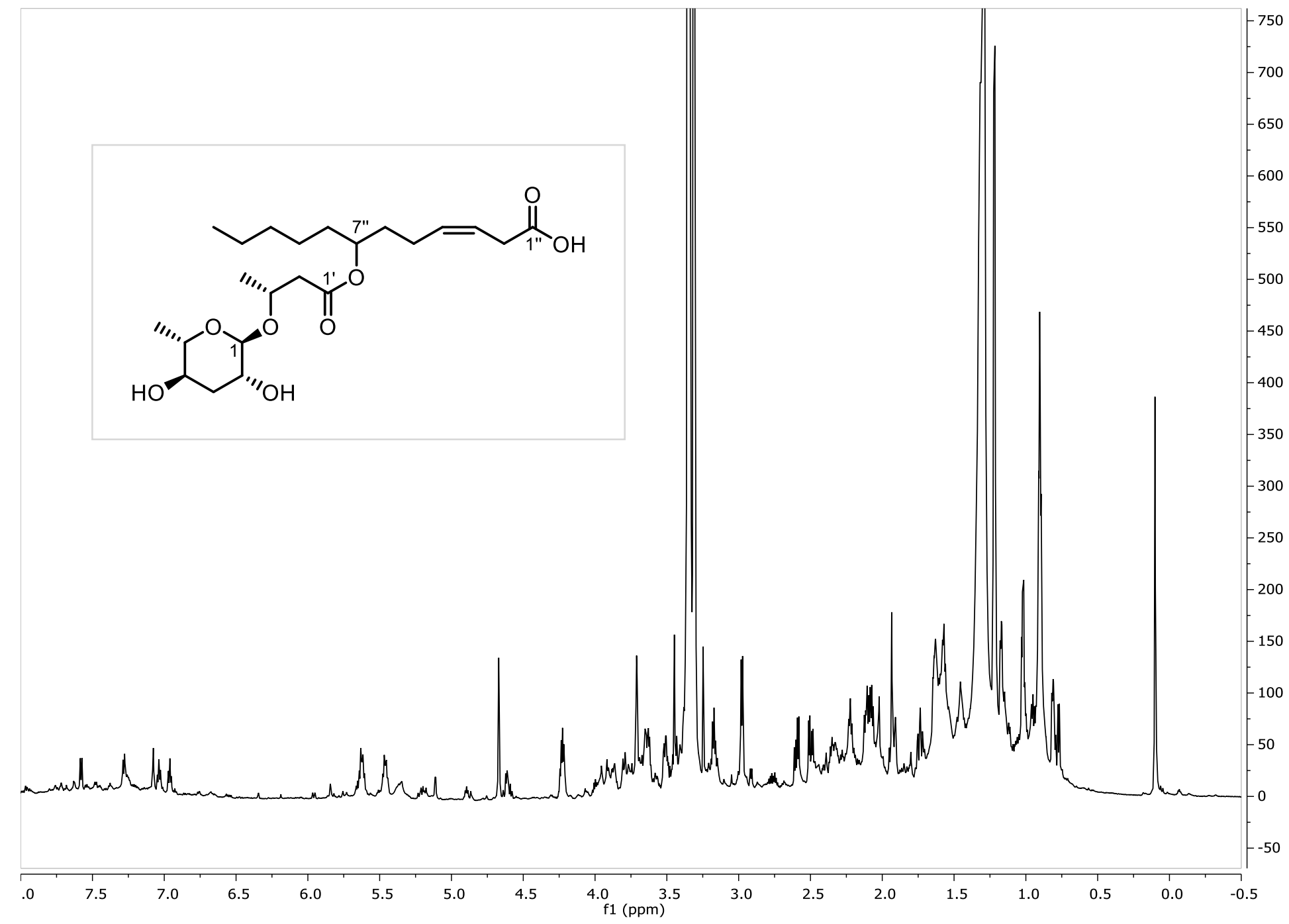


Figure S33: HSQC $\left(700 \mathrm{MHz}, \mathrm{CD}_{3} \mathrm{OD}\right)$ of natural asc-C4-C12:1 (fasc\#2, 4) from C. remanei.

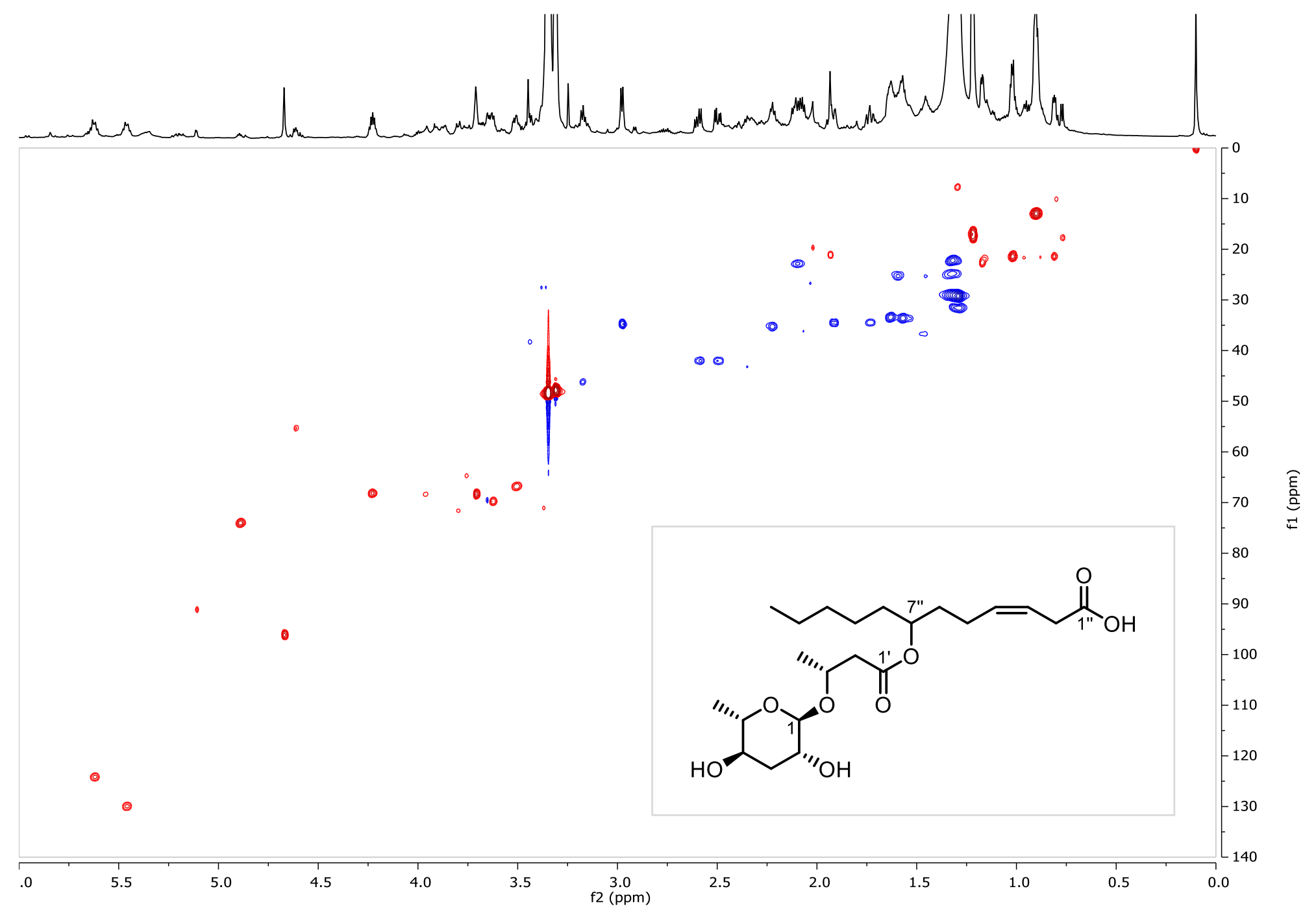


Figure S34: $\mathrm{HMBC}\left(700 \mathrm{MHz}, \mathrm{CD}_{3} \mathrm{OD}\right)$ of natural asc-C4-C12:1 (fasc\#2, 4) from C. remanei.

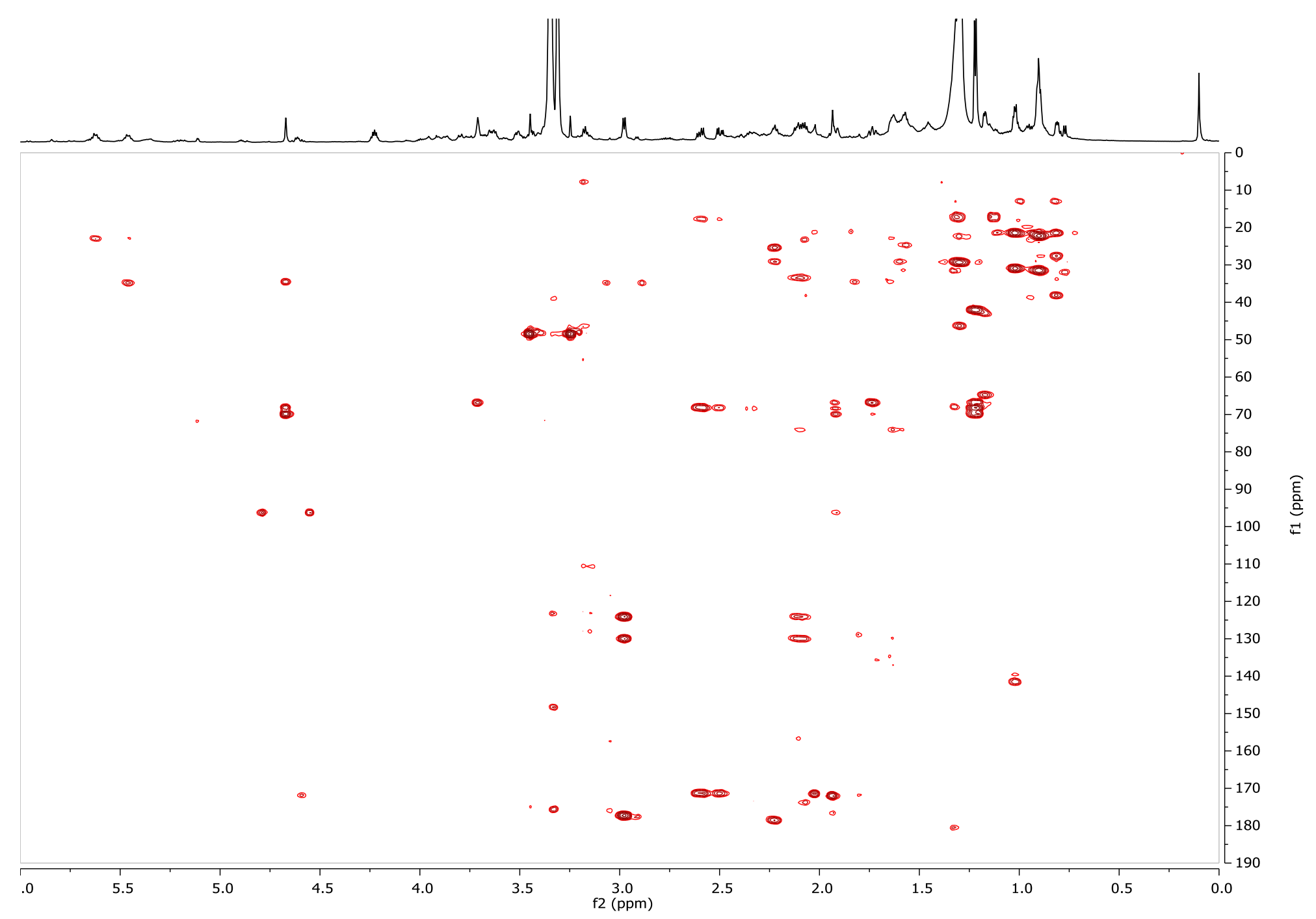


Figure S35: ${ }^{1} \mathrm{H}$ NMR $\left(700 \mathrm{MHz}, \mathrm{CD}_{3} \mathrm{OD}\right)$ of natural asc-C4-C8 (fasc\#4, 7) from C. latens.

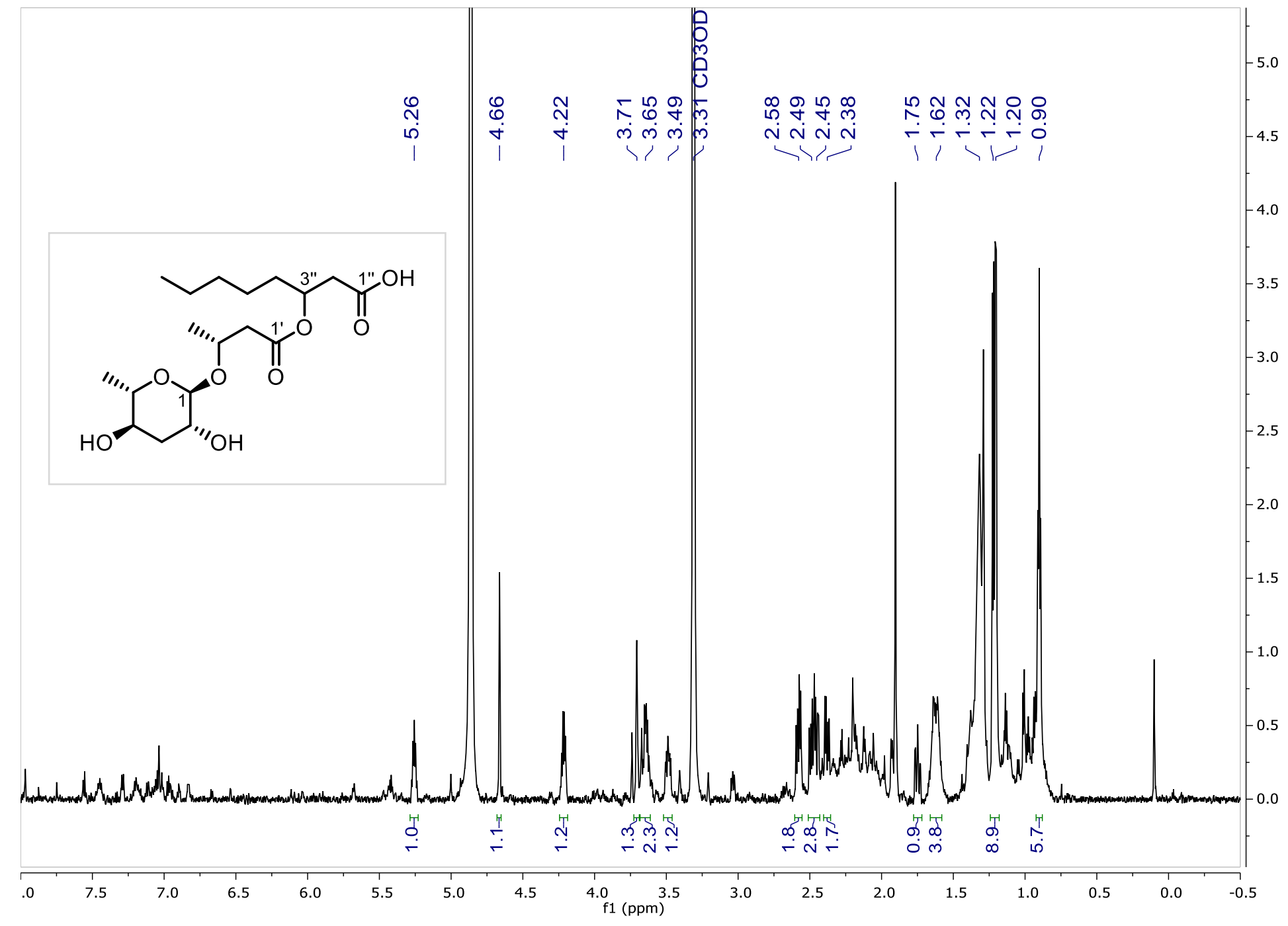


Figure S36: ${ }^{1} \mathrm{H}$ NMR $\left(700 \mathrm{MHz}, \mathrm{CD}_{3} \mathrm{OD}\right)$ of natural asc-C4-C8 (fasc\#4, 7) from C. latens.

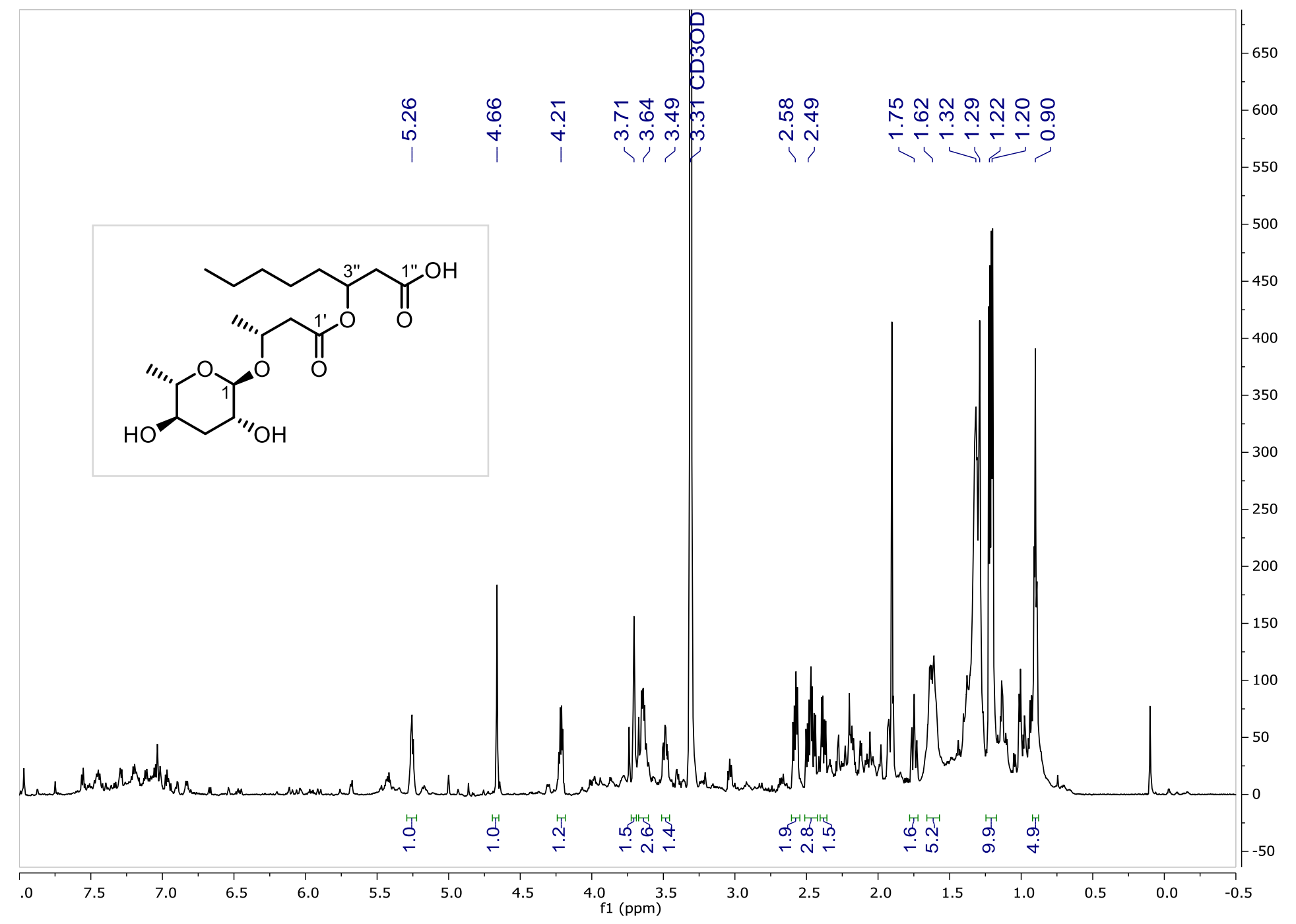


Figure S37: dqf-COSY (700 MHz, $\left.\mathrm{CD}_{3} \mathrm{OD}\right)$ of natural asc-C4-C8 (fasc\#4, 7) from C. latens.

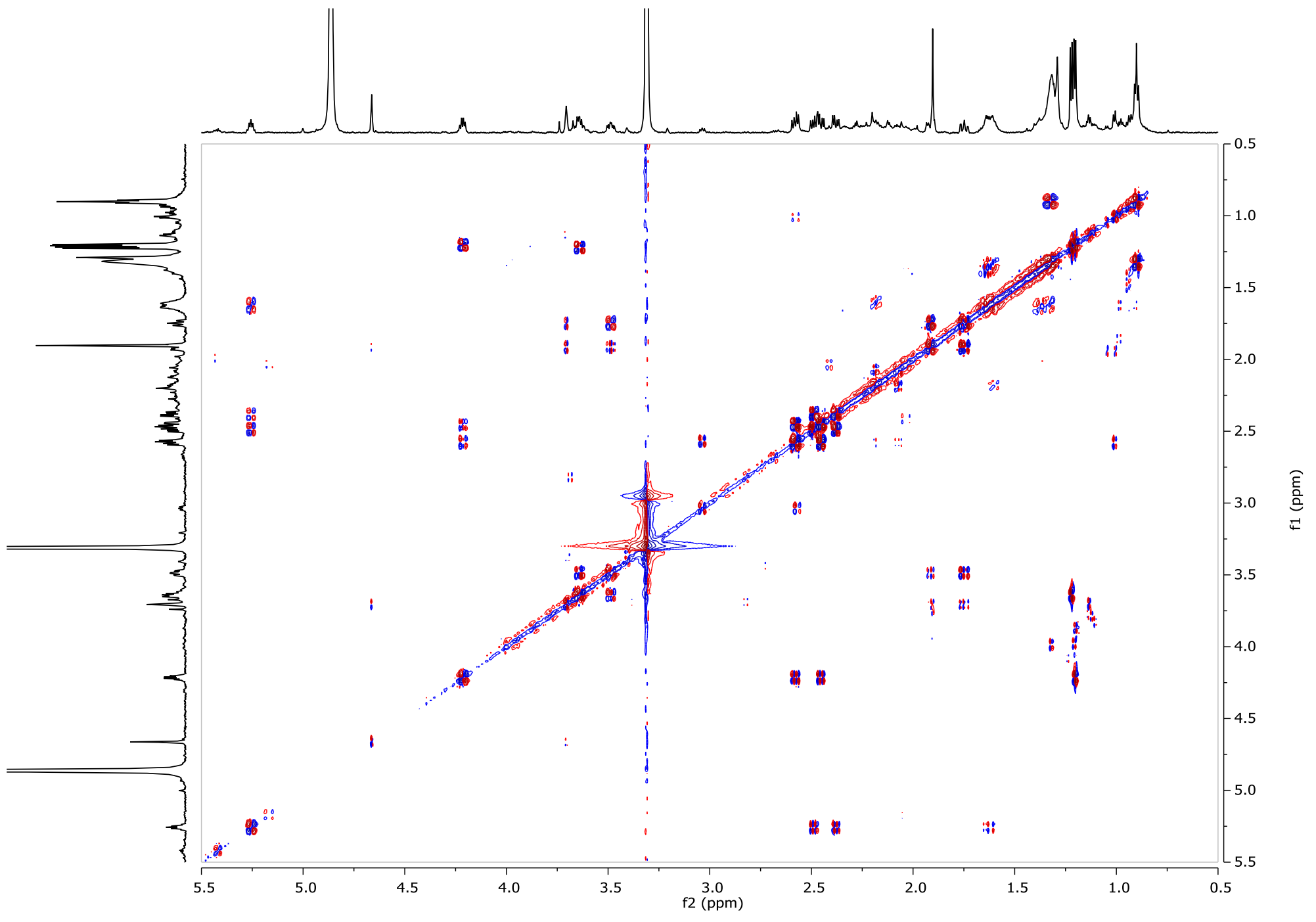


Figure S38: HSQC (700 MHz, $\left.\mathrm{CD}_{3} \mathrm{OD}\right)$ of natural asc-C4-C8 (fasc\#4, 7) from C. latens.

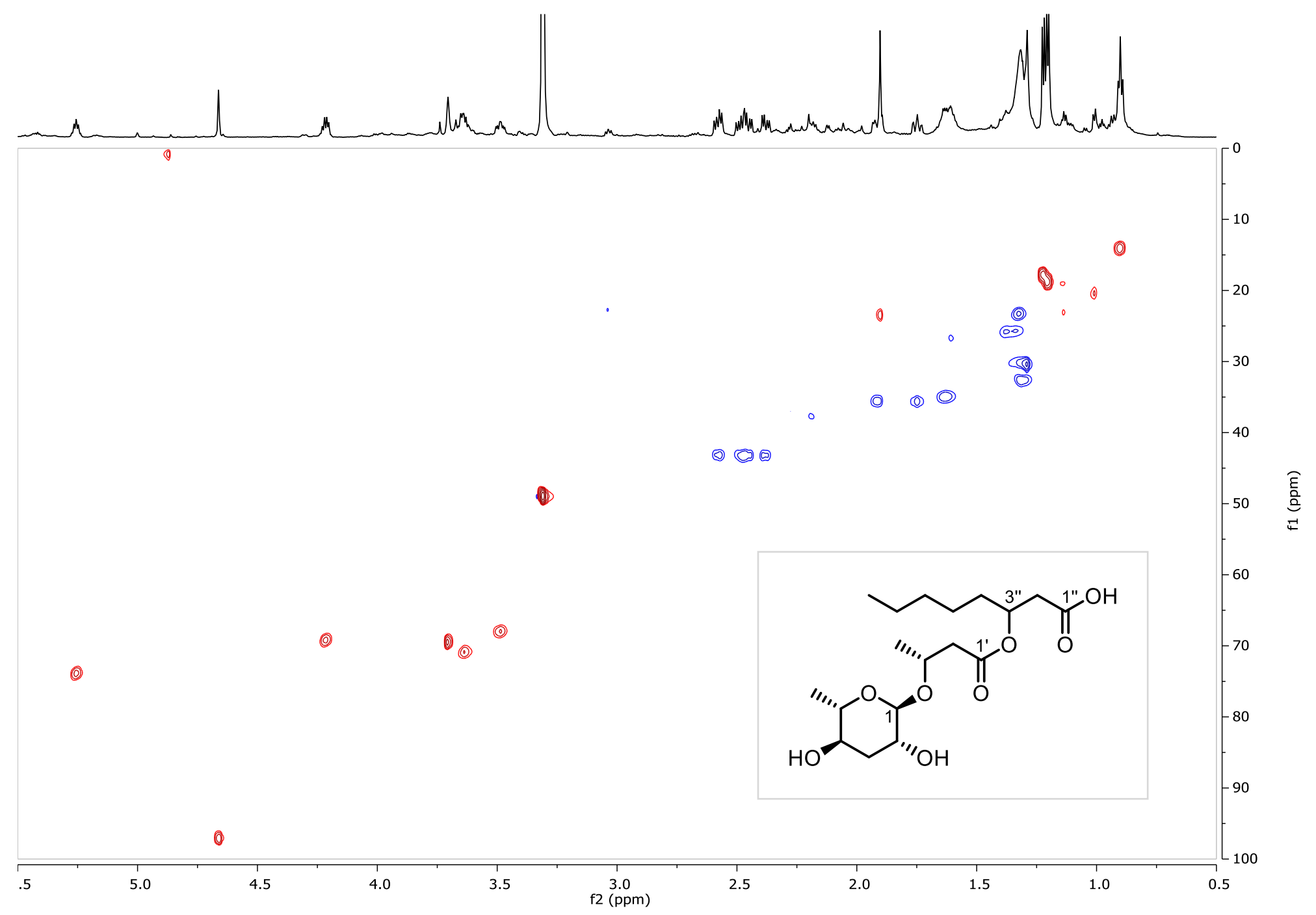


Figure S39: $\mathrm{HMBC}\left(700 \mathrm{MHz}, \mathrm{CD}_{3} \mathrm{OD}\right)$ of natural asc-C4-C8 (fasc\#4, 7) from C. latens.

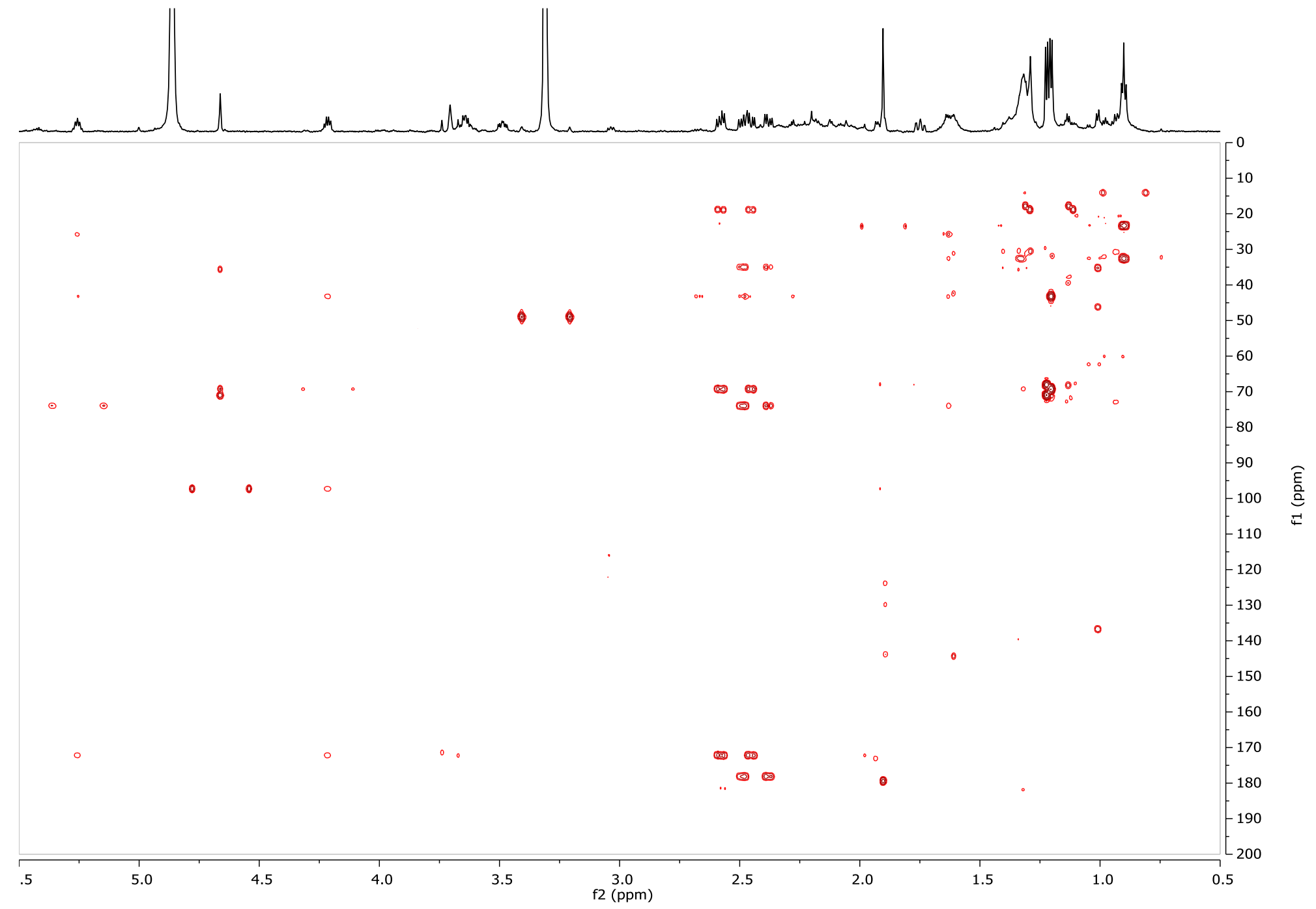


Figure S40: ${ }^{1} \mathrm{H}$ NMR $\left(700 \mathrm{MHz}, \mathrm{CD}_{3} \mathrm{OD}\right.$ ) of natural asc-C4-C6 (fasc\#5) from C. latens.

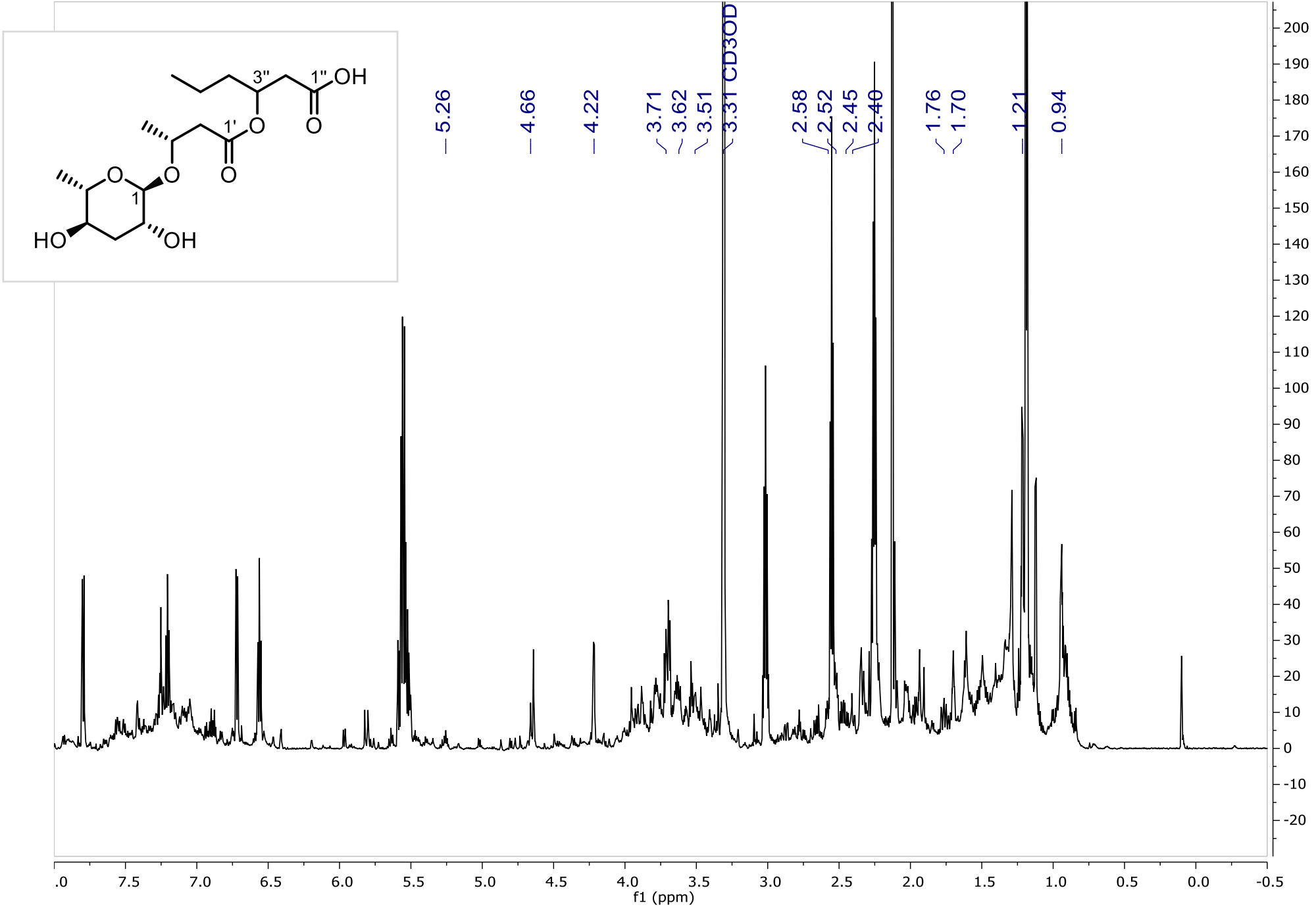


Figure S41: dqf-COSY (700 MHz, $\mathrm{CD}_{3} \mathrm{OD}$ ) of natural asc-C4-C6 (fasc\#5) from C. latens.

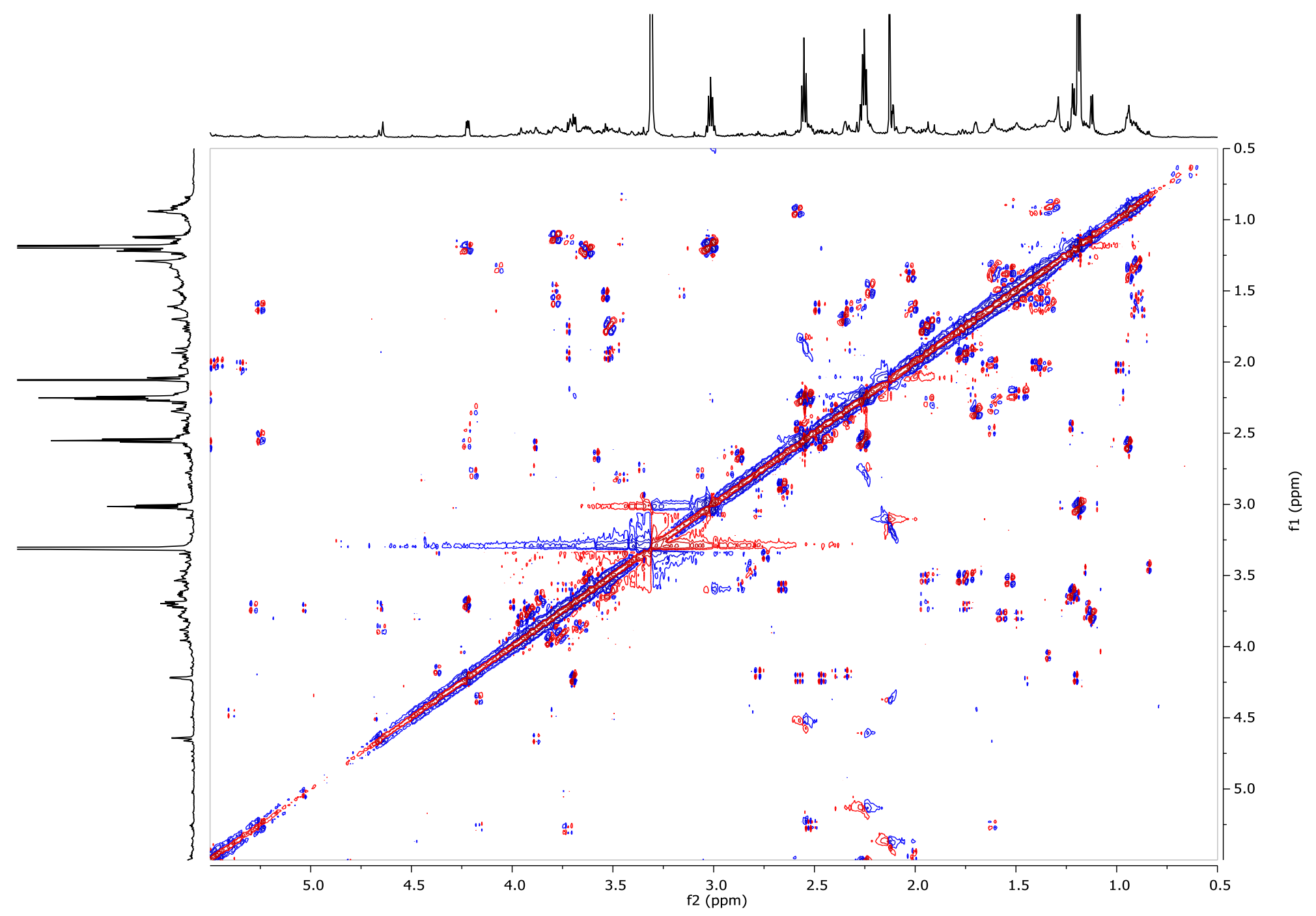


Figure S42: HSQC (700 MHz, $\mathrm{CD}_{3} \mathrm{OD}$ ) of natural asc-C4-C6 (fasc\#5) from C. latens.

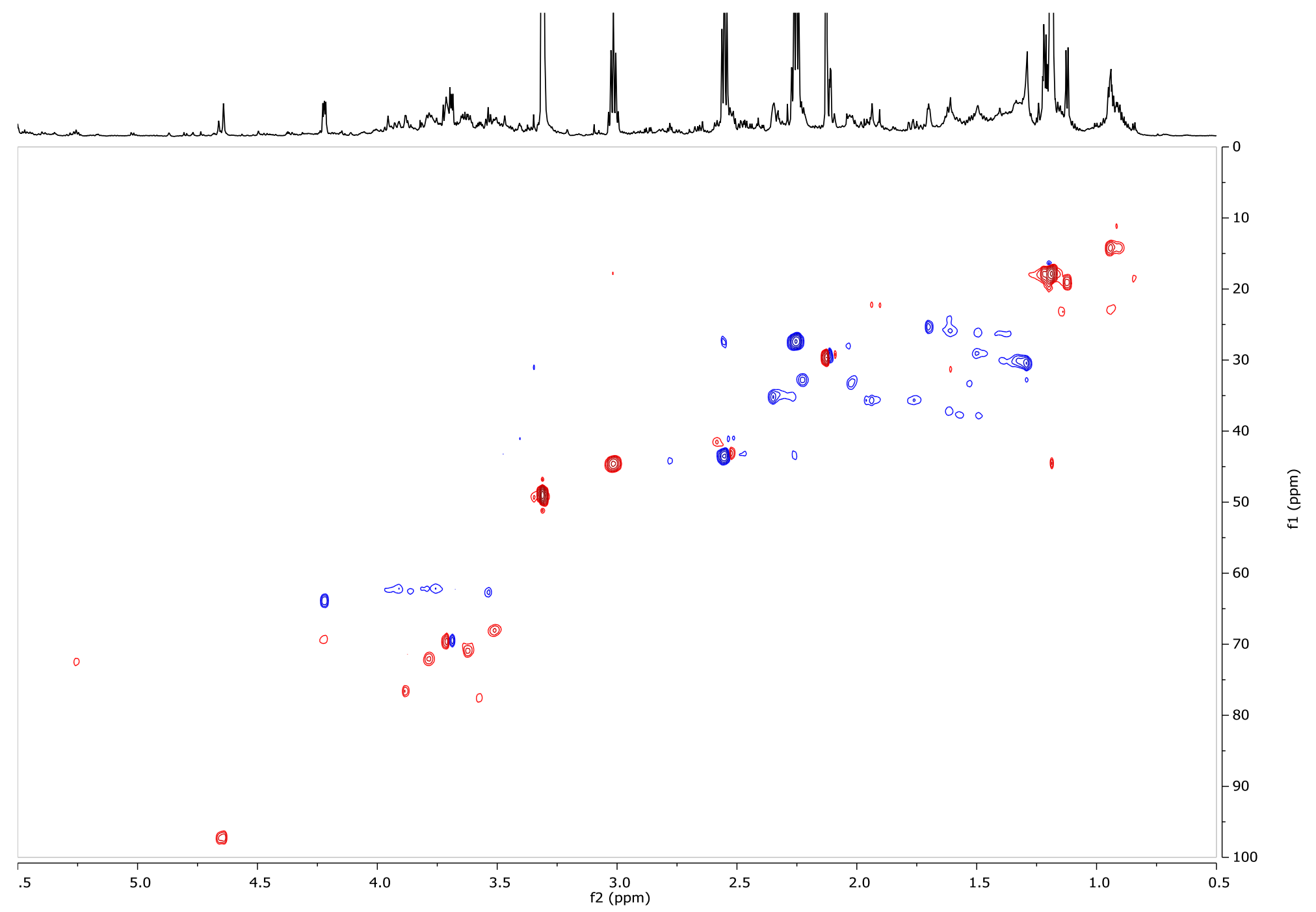


Figure S43: ${ }^{1} \mathrm{H}$ NMR (400 MHz, $\mathrm{CD}_{3} \mathrm{OD}$ ) of natural 7-hydroxy-3,4-cis-methylene decanoic acid (8).

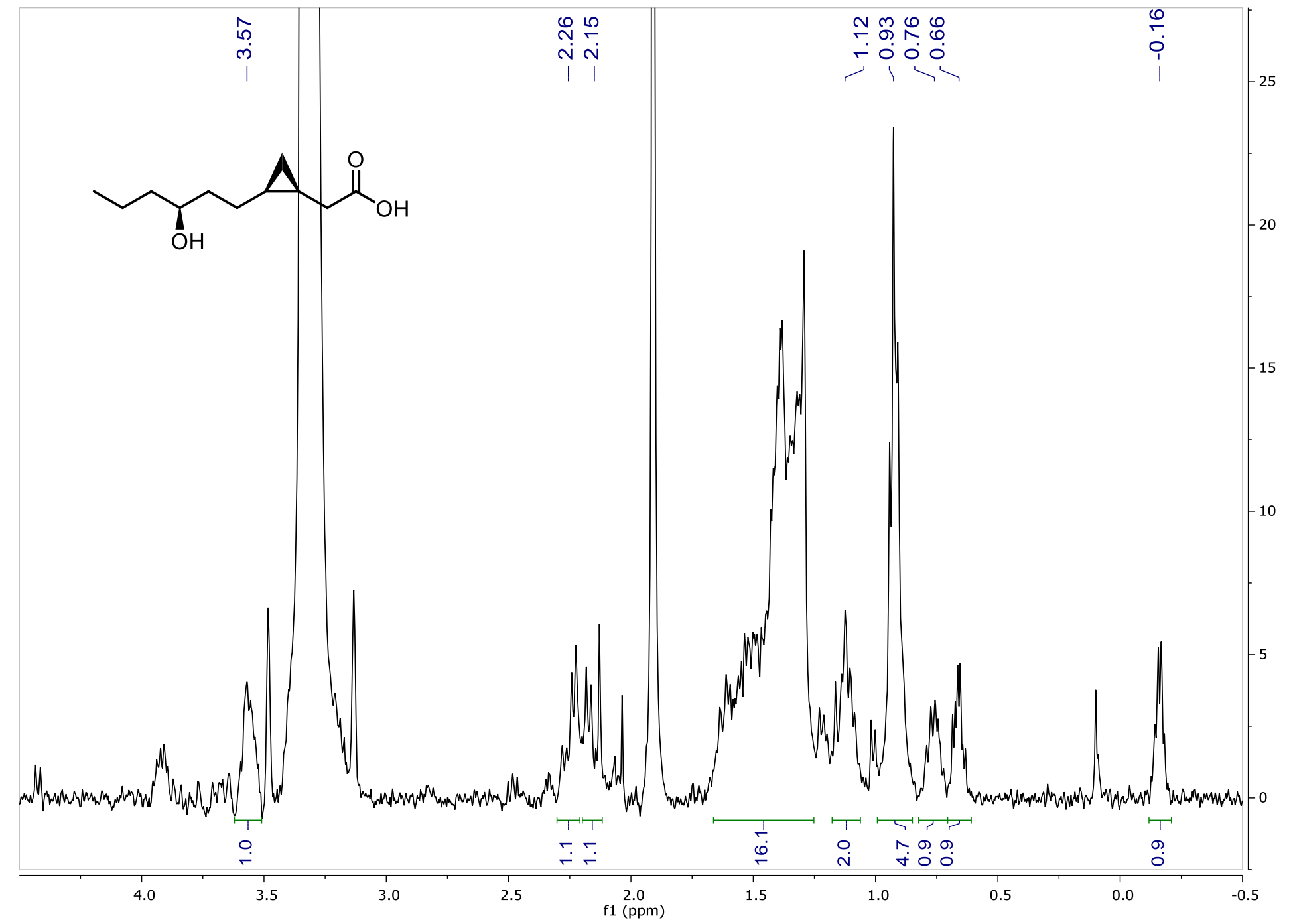


Figure S44: COSY spectrum (400 MHz, $\left.\mathrm{CD}_{3} \mathrm{OD}\right)$ of natural 7-hydroxy-3,4-cis-methylene decanoic acid (8).

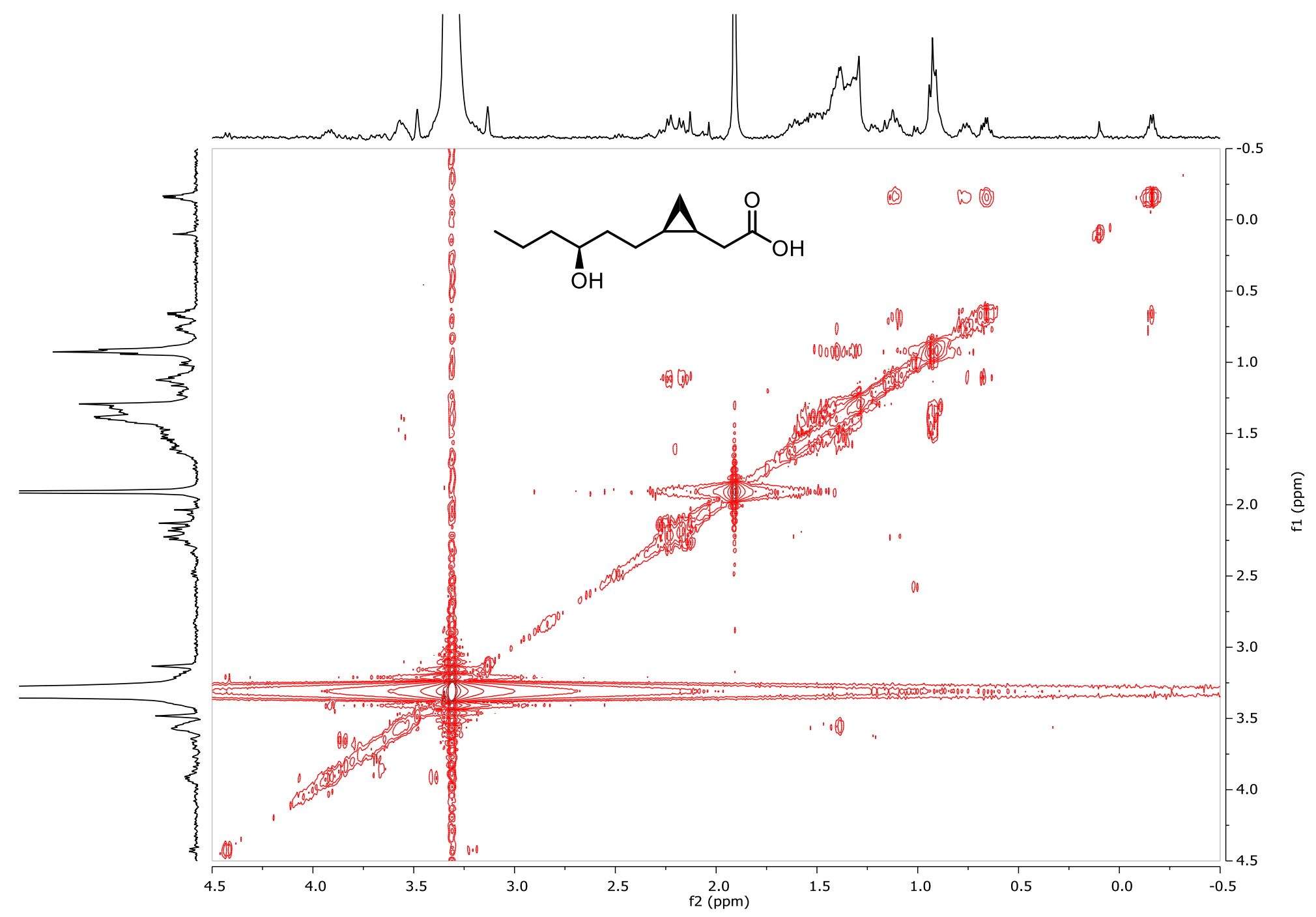


Figure S45: ${ }^{1} \mathrm{H}$ NMR (400 MHz, $\left.\mathrm{CD}_{3} \mathrm{OD}\right)$ of natural 10-hydroxy-3,4-cis-methylene decanoic acid.

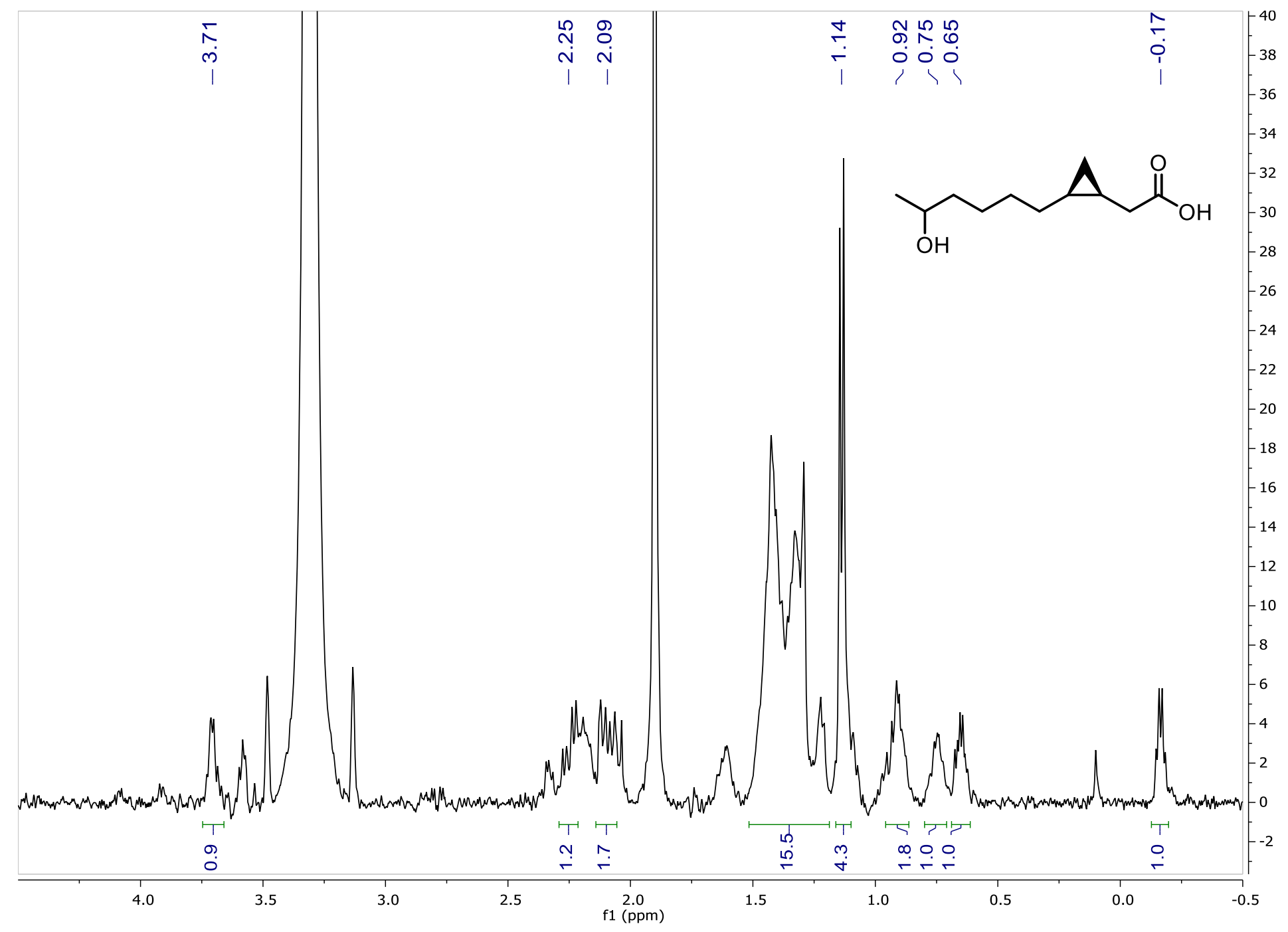


Figure S46: COSY spectrum (400 MHz, $\left.\mathrm{CD}_{3} \mathrm{OD}\right)$ of natural 10-hydroxy-3,4-cis-methylene decanoic acid.

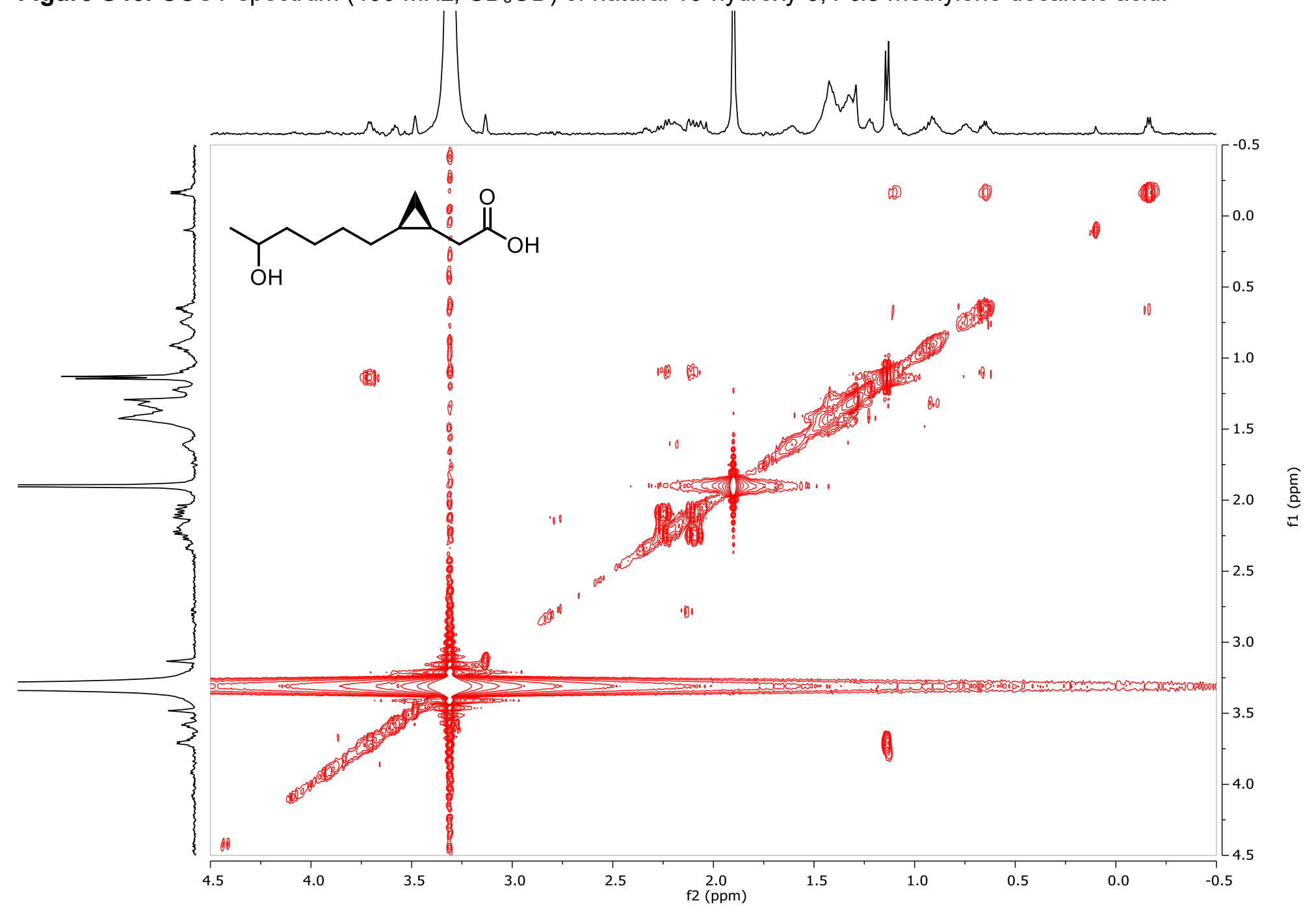


Figure S47: ${ }^{1} \mathrm{H}$ NMR (400 MHz, $\mathrm{CDCl}_{3}$ ) of methyl 7-hydroxy-cis-3,4-metylene decanoate (9).

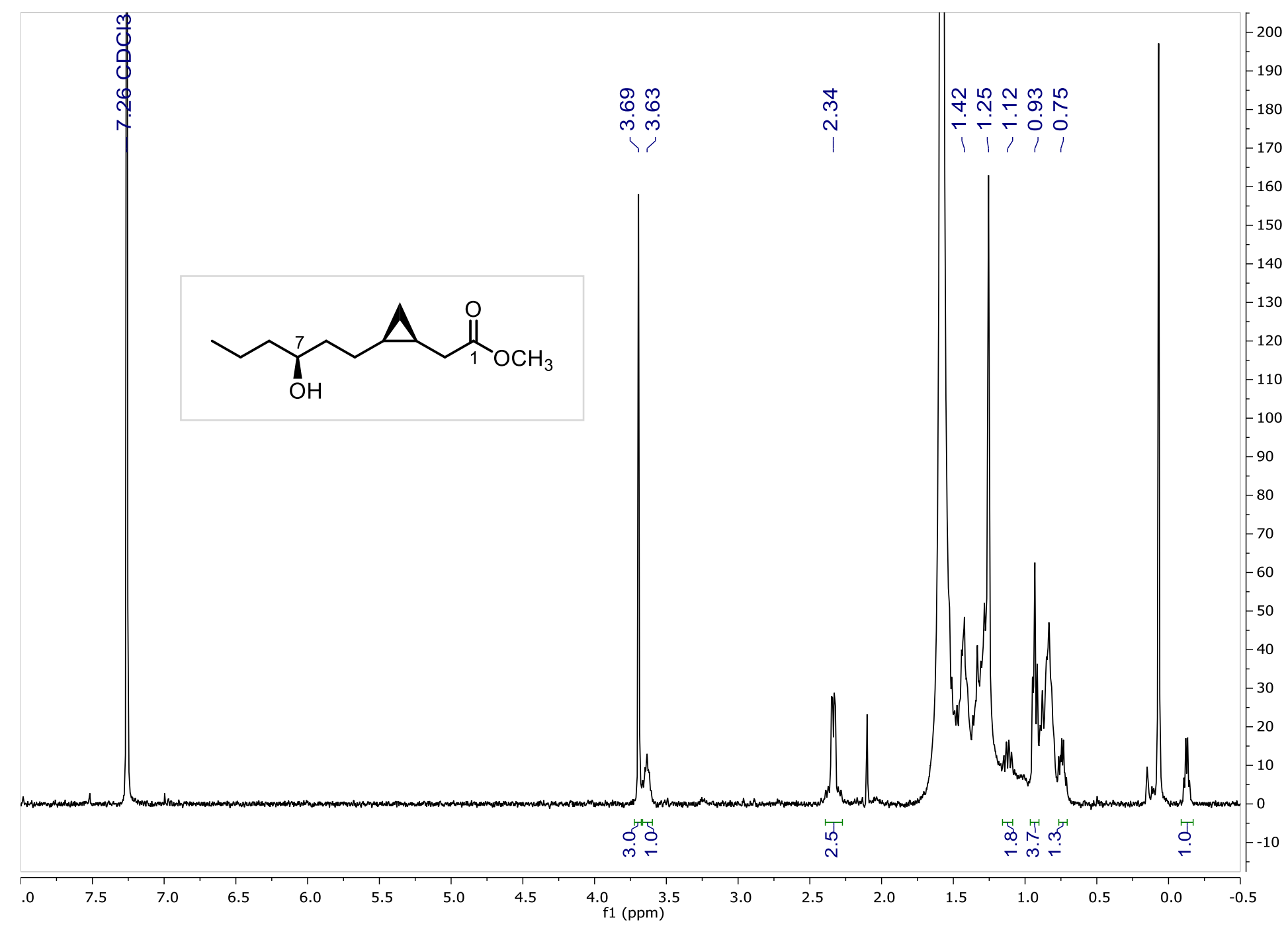


Figure S48: $d q f-\mathrm{COSY}$ spectrum (400 MHz, $\mathrm{CDCl}_{3}$ ) of methyl 7-hydroxy-cis-3,4-metylene decanoate (9).

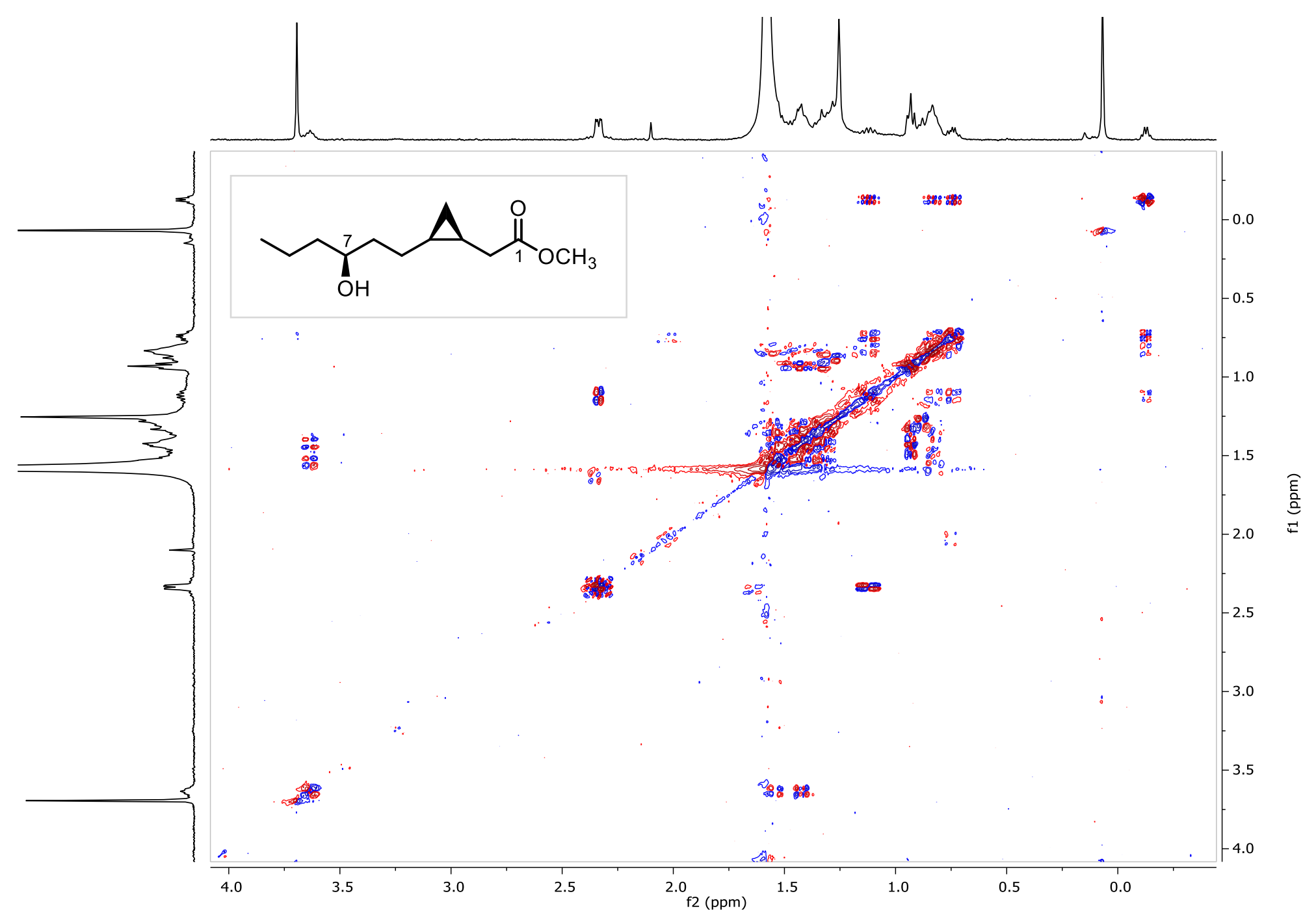


Figure S49: ${ }^{1} \mathrm{H} \mathrm{NMR}\left(700 \mathrm{MHz}, \mathrm{CDCl}_{3}, 6.5 \% \mathrm{C}_{5} \mathrm{D}_{5} \mathrm{~N}\right)$ of methyl 7-hydroxy-cis-3,4-metylene decanoate (9).

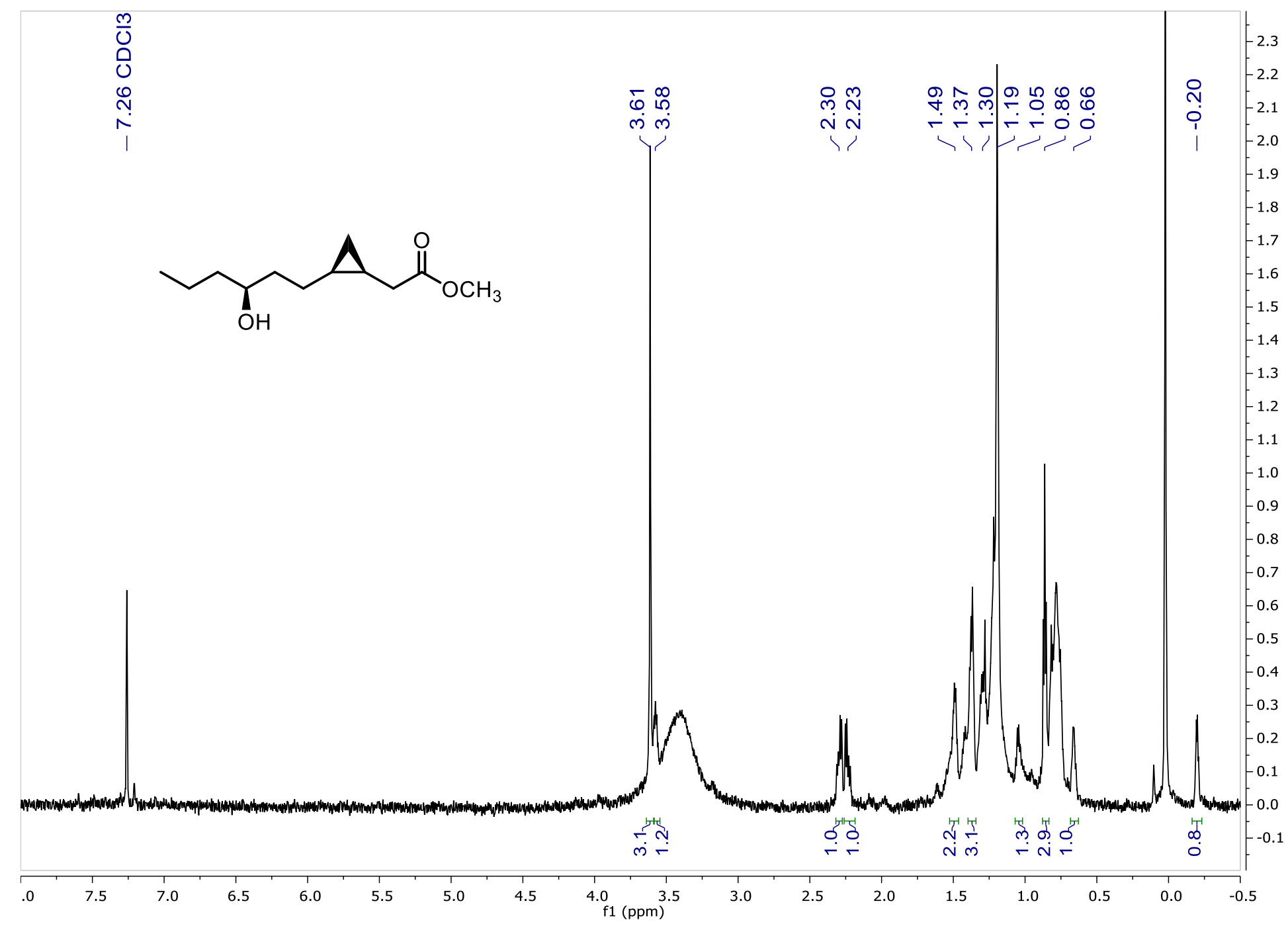


Figure S50a: SELTOCSY (700 MHz, $\left.\mathrm{CDCl}_{3}, 6.5 \% \mathrm{C}_{5} \mathrm{D}_{5} \mathrm{~N}\right)$ of methyl 7-hydroxy-cis-3,4-metylene decanoate (9).

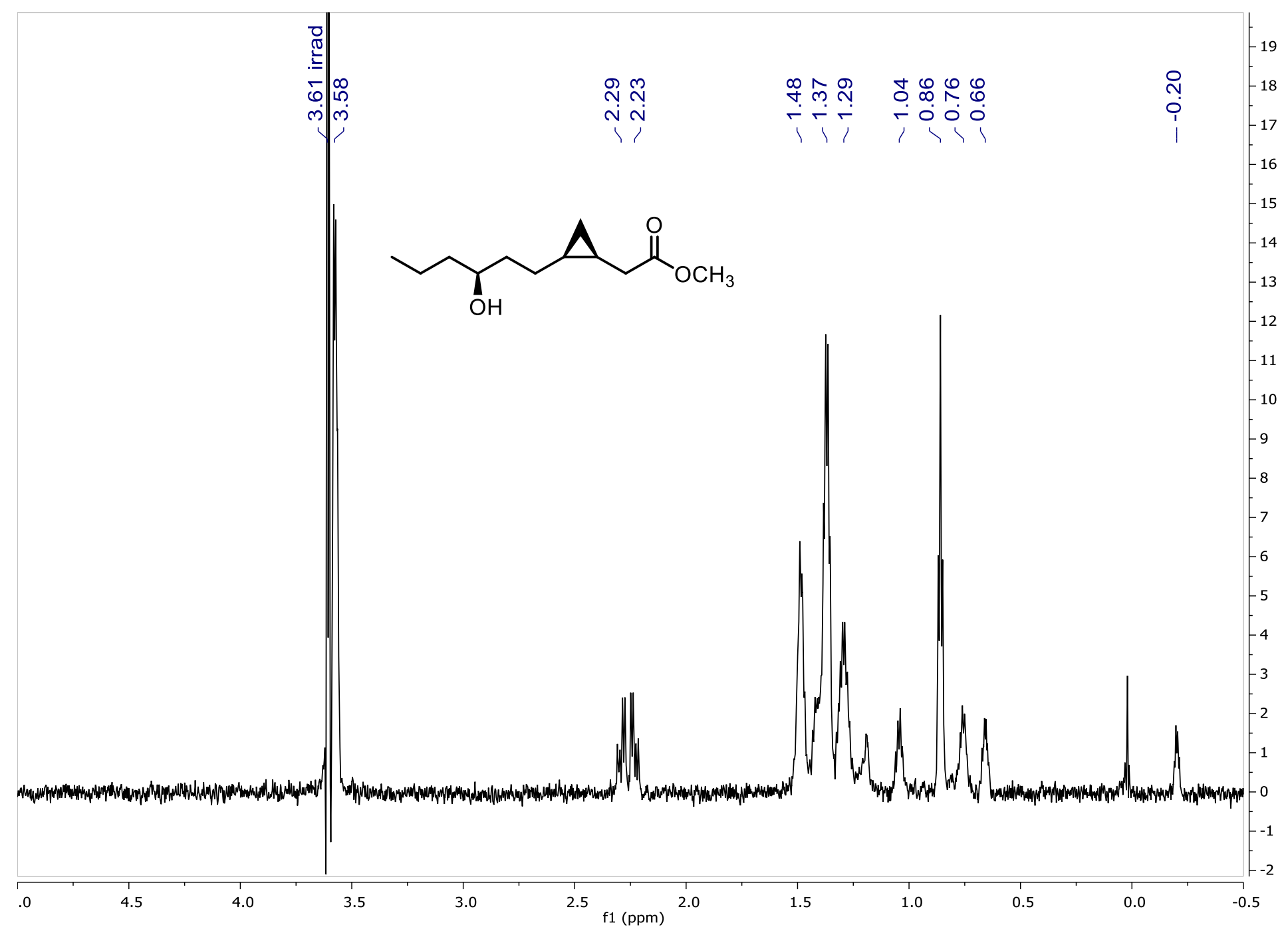


Figure S50b: SELTOCSY (700 MHz, $\left.\mathrm{CDCl}_{3}, 6.5 \% \mathrm{C}_{5} \mathrm{D}_{5} \mathrm{~N}\right)$ of methyl 7-hydroxy-cis-3,4-metylene decanoate (9).

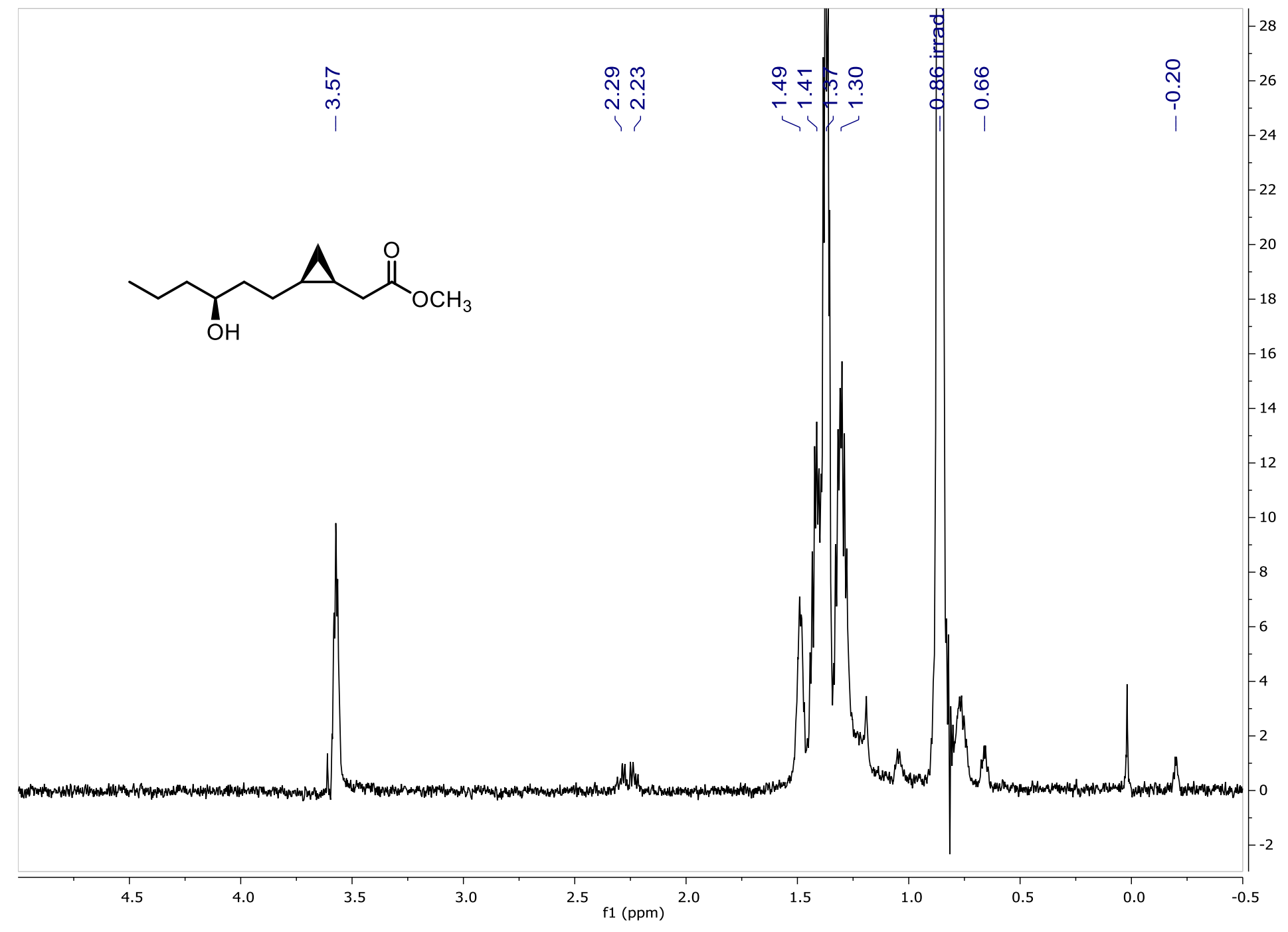


Figure S50c: SELTOCSY (700 MHz, $\left.\mathrm{CDCl}_{3}, 6.5 \% \mathrm{C}_{5} \mathrm{D}_{5} \mathrm{~N}\right)$ of methyl 7-hydroxy-cis-3,4-metylene decanoate (9).

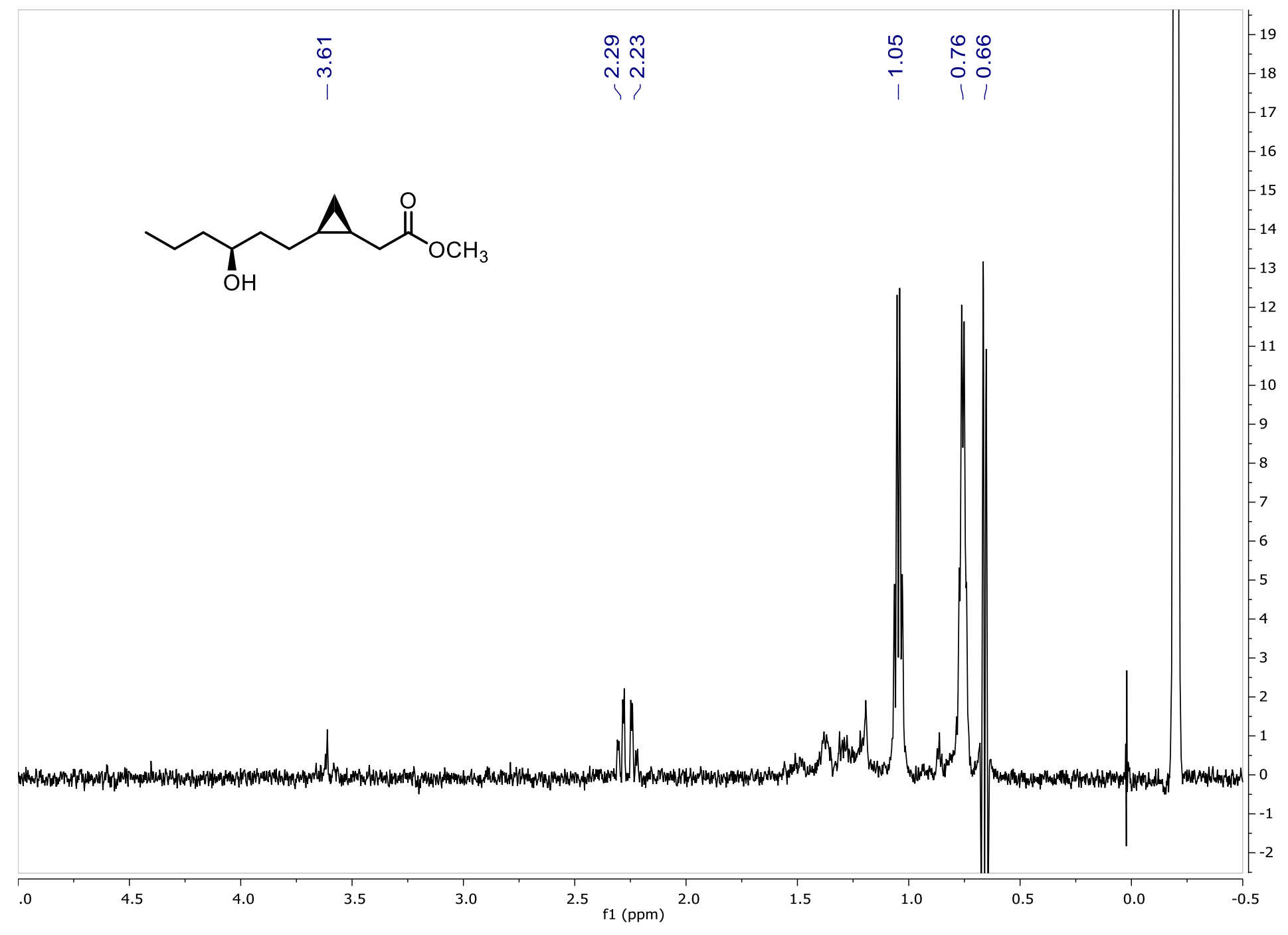


Figure S51: dqf-COSY $\left(700 \mathrm{MHz}, \mathrm{CDCl}_{3}, 6.5 \% \mathrm{C}_{5} \mathrm{D}_{5} \mathrm{~N}\right)$ of methyl 7-hydroxy-cis-3,4-metylene decanoate (9).

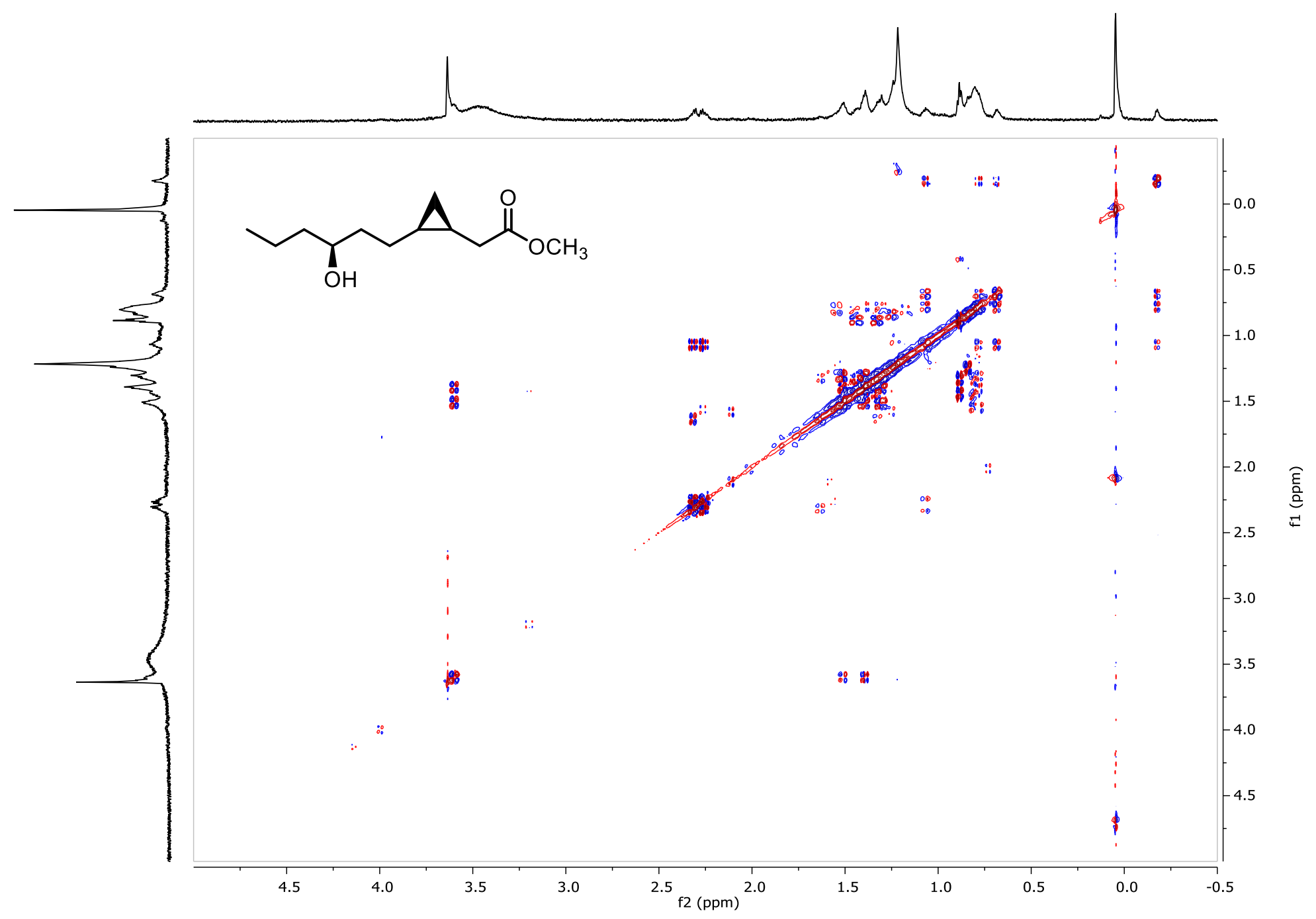


Figure S52: HSQC (700 MHz, $\left.\mathrm{CDCl}_{3}, 6.5 \% \mathrm{C}_{5} \mathrm{D}_{5} \mathrm{~N}\right)$ of methyl 7-hydroxy-cis-3,4-metylene decanoate (9).

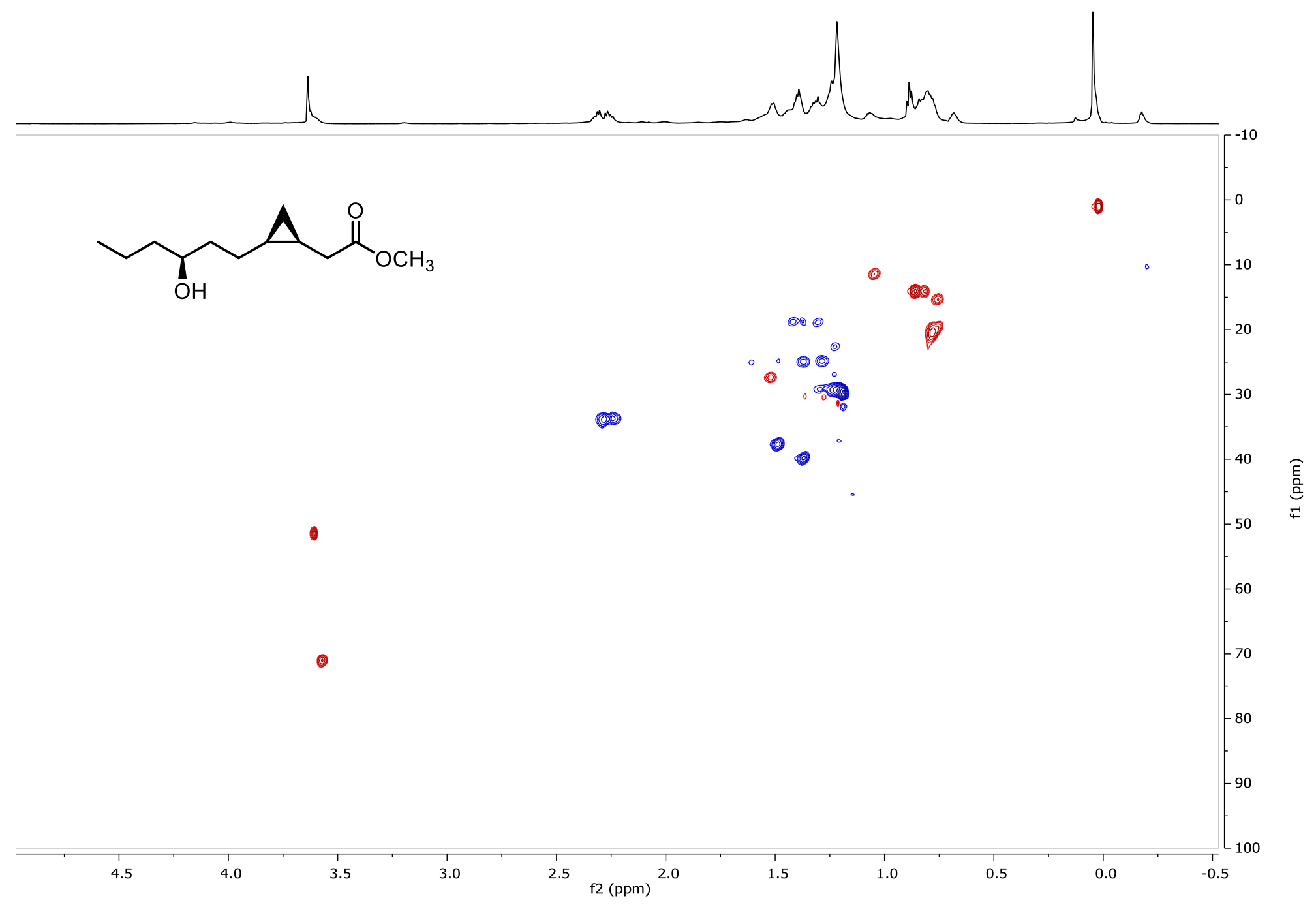


Figure S53: ${ }^{1} \mathrm{H}$ NMR $\left(700 \mathrm{MHz}, \mathrm{CDCl}_{3}, 6.5 \% \mathrm{C}_{5} \mathrm{D}_{5} \mathrm{~N}\right)$ of methyl 7-hydroxy-cis-3,4-methylene decanoate $(R)-\mathrm{MTPA}$ ester $((R)-10)$.

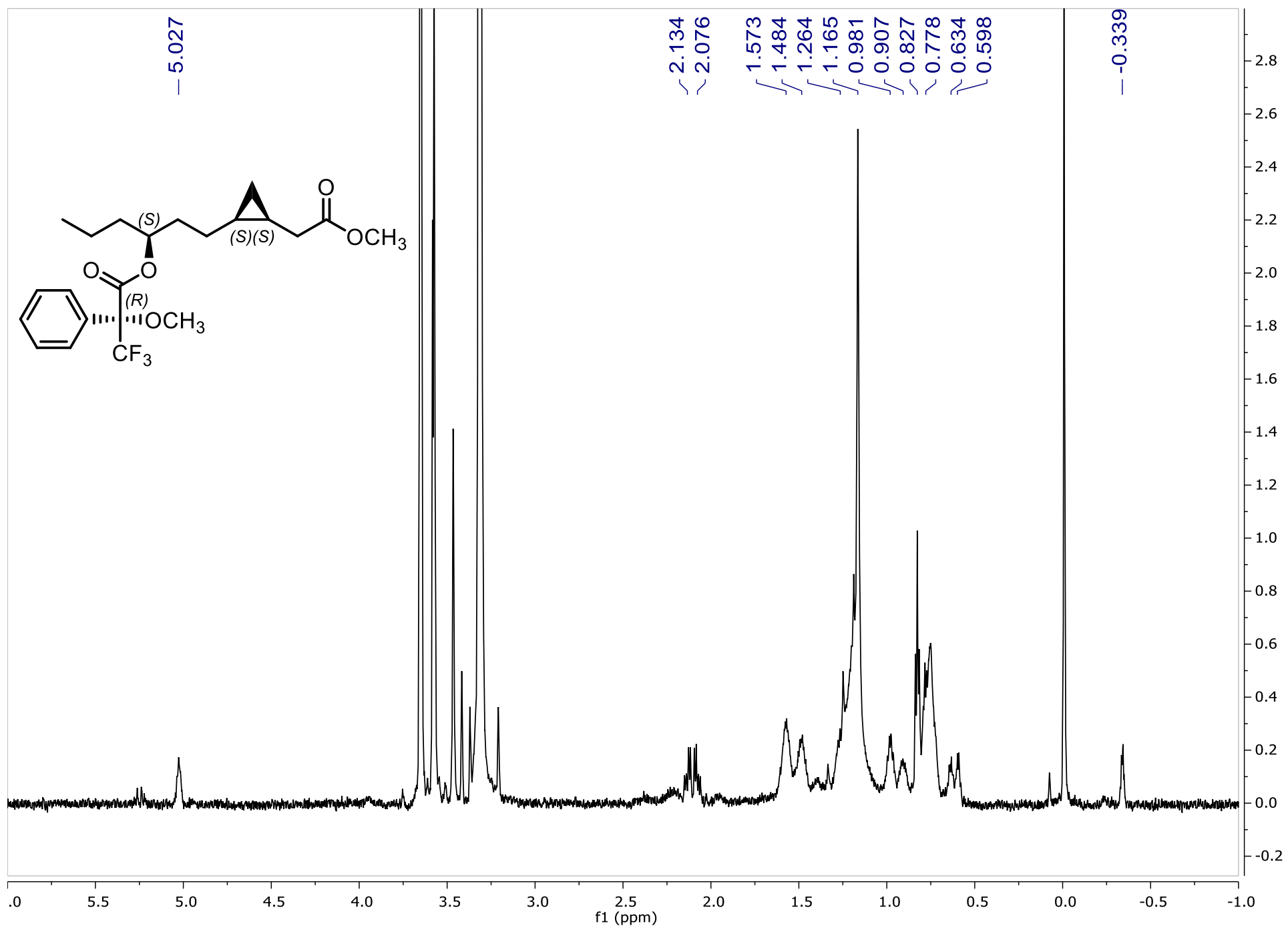


Figure S54a: SELTOCSY $\left(700 \mathrm{MHz}, \mathrm{CDCl}_{3}, 6.5 \% \mathrm{C}_{5} \mathrm{D}_{5} \mathrm{~N}\right)$ of methyl 7-hydroxy-cis-3,4-methylene decanoate $(R)-\mathrm{MTPA}$ ester $((R)-$ $10)$.

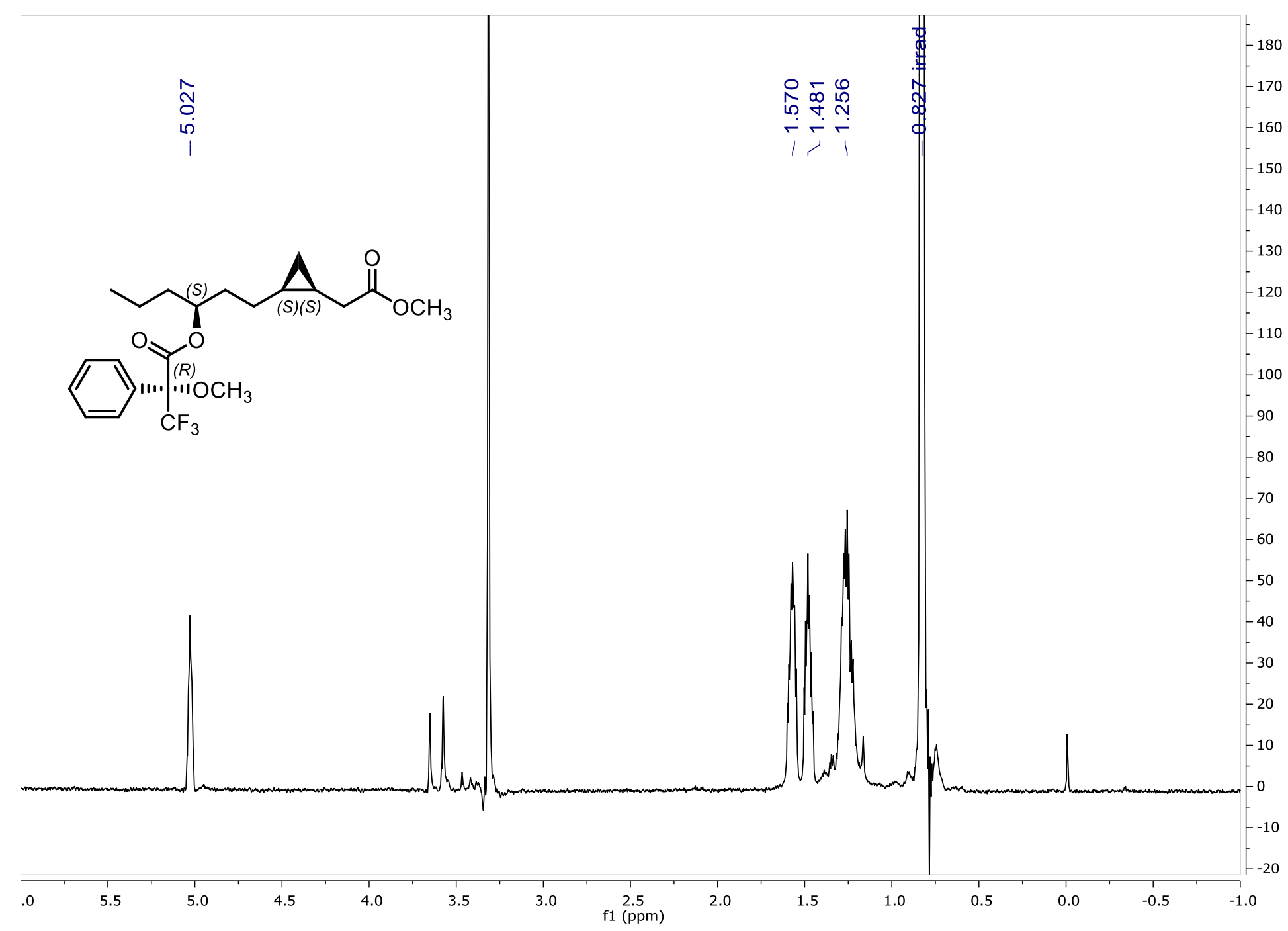


Figure S54b: SELTOCSY $\left(700 \mathrm{MHz}, \mathrm{CDCl}_{3}, 6.5 \% \mathrm{C}_{5} \mathrm{D}_{5} \mathrm{~N}\right)$ of methyl 7-hydroxy-cis-3,4-methylene decanoate $(R)$-MTPA ester $((R)$ $10)$.

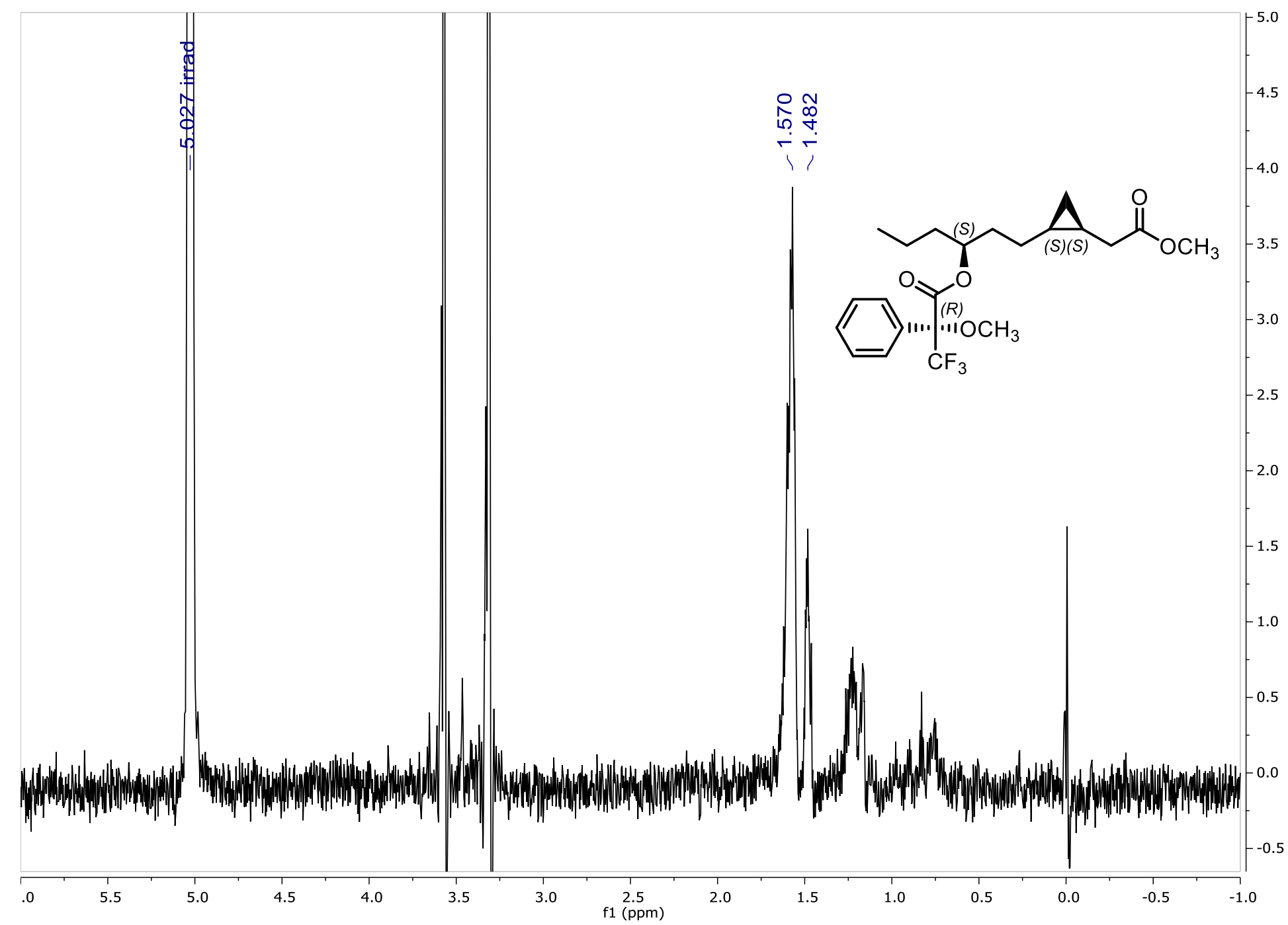


Figure S54c: SELTOCSY $\left(700 \mathrm{MHz}, \mathrm{CDCl}_{3}, 6.5 \% \mathrm{C}_{5} \mathrm{D}_{5} \mathrm{~N}\right)$ of methyl 7-hydroxy-cis-3,4-methylene decanoate $(R)-\mathrm{MTPA}$ ester $((R)-$ $10)$.

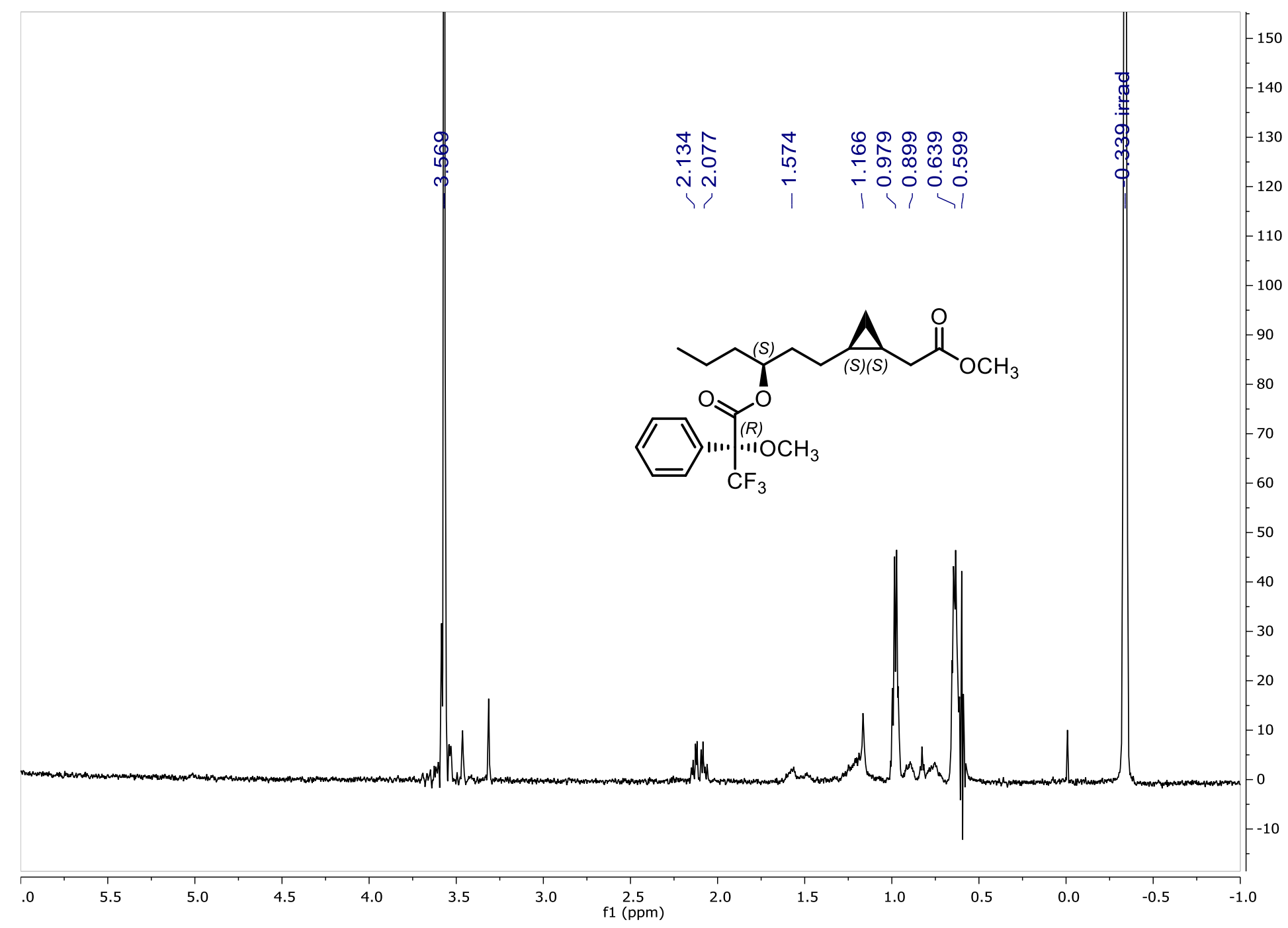


Figure S54d: SELTOCSY $\left(700 \mathrm{MHz}, \mathrm{CDCl}_{3}, 6.5 \% \mathrm{C}_{5} \mathrm{D}_{5} \mathrm{~N}\right)$ of methyl 7-hydroxy-cis-3,4-methylene decanoate $(R)$-MTPA ester $((R)$ 10).

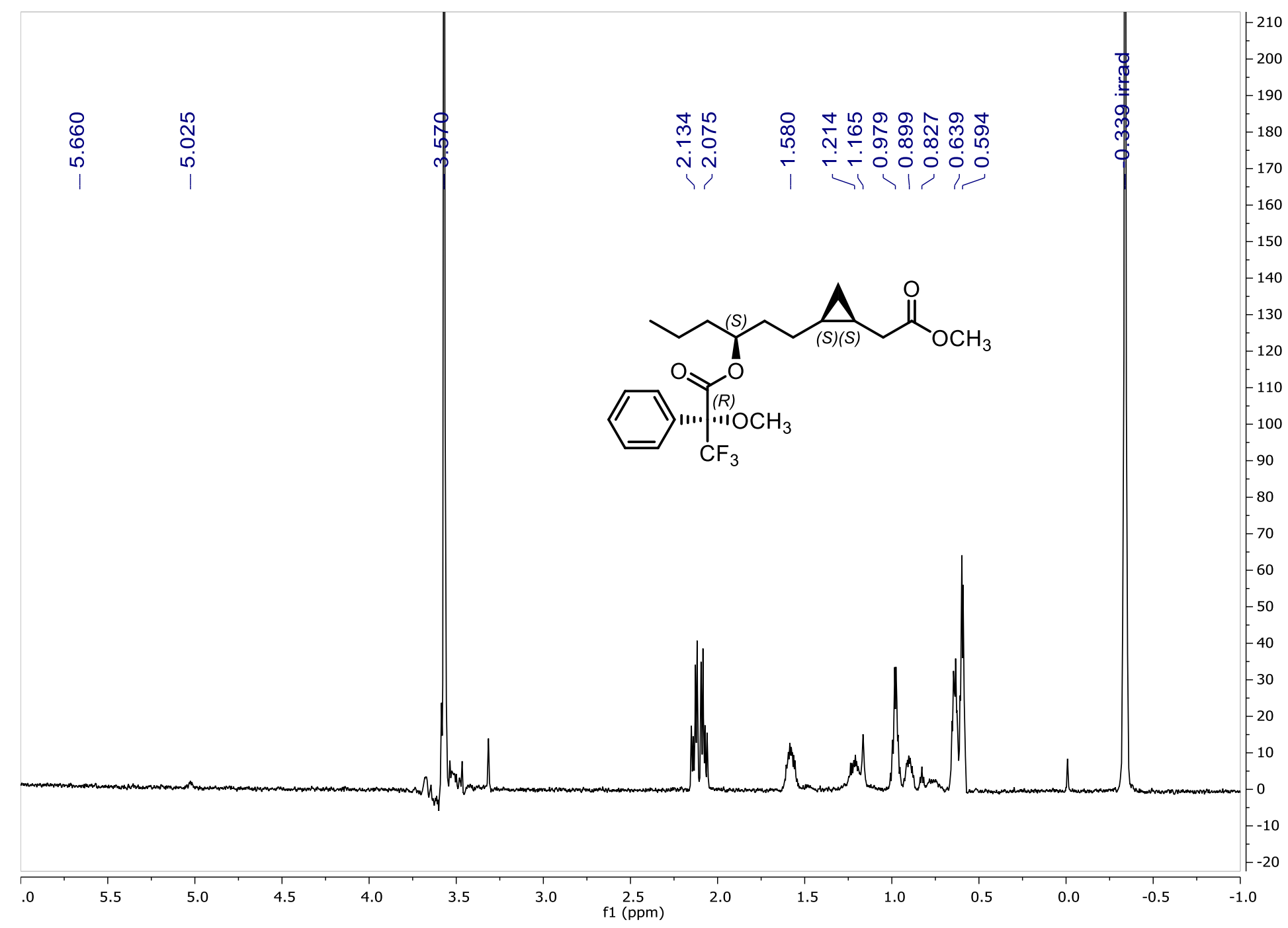


Figure S55: dqf-COSY (700 MHz, $\left.\mathrm{CDCl}_{3}, 6.5 \% \mathrm{C}_{5} \mathrm{D}_{5} \mathrm{~N}\right)$ of methyl 7-hydroxy-cis-3,4-methylene decanoate $(R)$-MTPA ester ((R)-10).

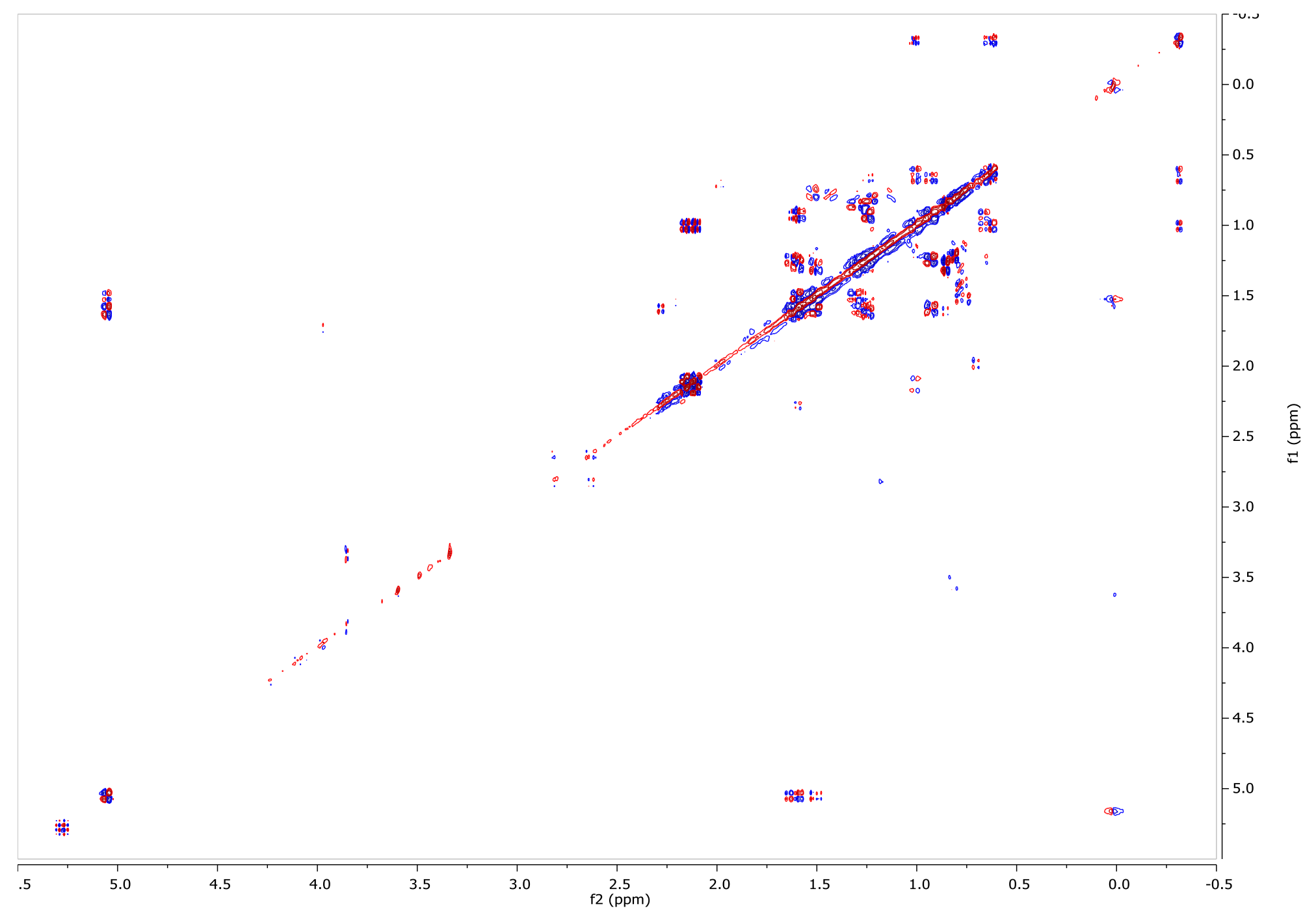


Figure S56: HSQC (700 MHz, $\left.\mathrm{CDCl}_{3}, 6.5 \% \mathrm{C}_{5} \mathrm{D}_{5} \mathrm{~N}\right)$ of methyl 7-hydroxy-cis-3,4-methylene decanoate $(R)$-MTPA ester $((R)-10)$.

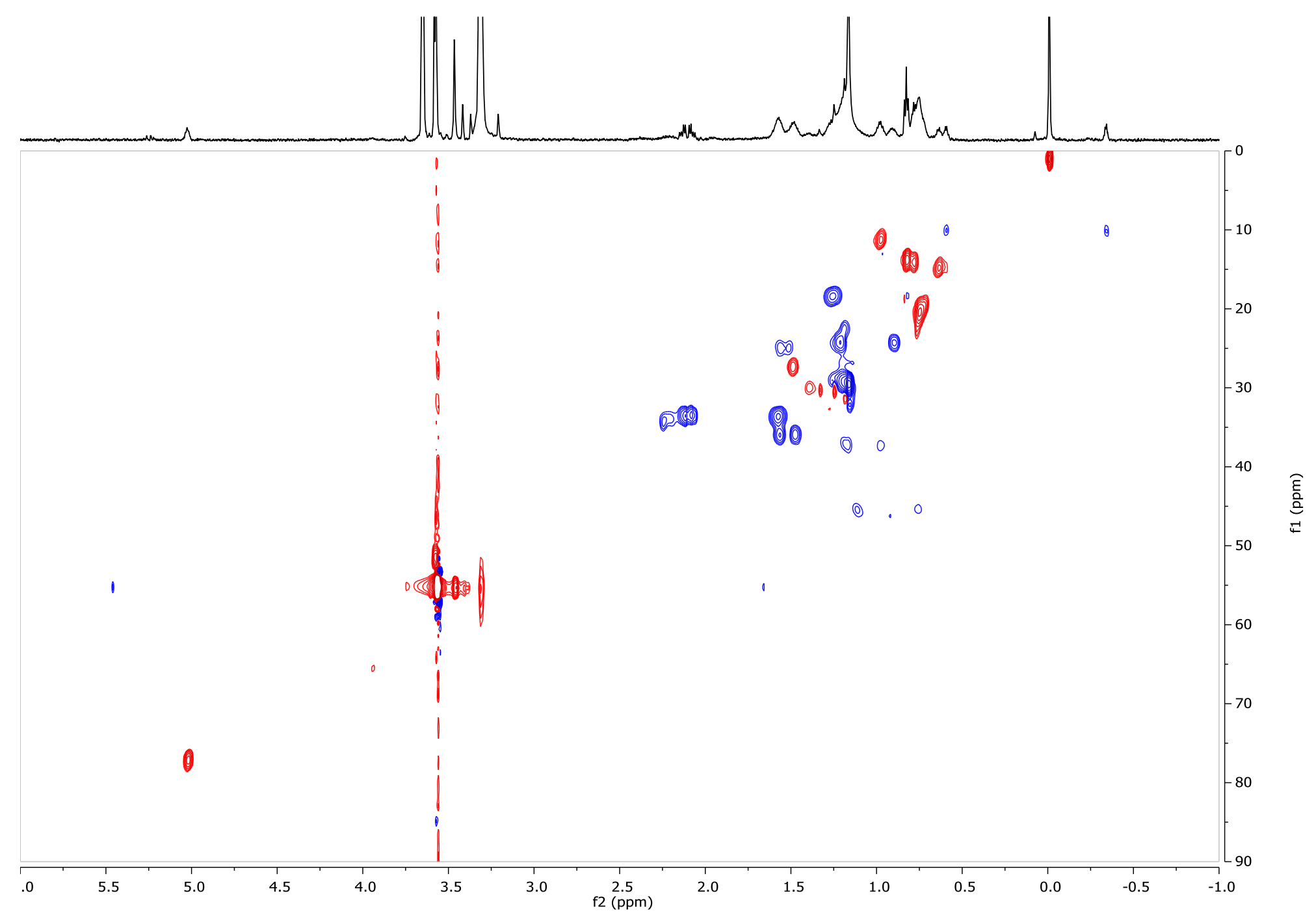


Figure S57: ${ }^{1} \mathrm{H}$ NMR $\left(700 \mathrm{MHz}, \mathrm{CDCl}_{3}, 6.5 \% \mathrm{C}_{5} \mathrm{D}_{5} \mathrm{~N}\right)$ of methyl 7-hydroxy-cis-3,4-methylene decanoate (S)-MTPA ester ((S)-10).

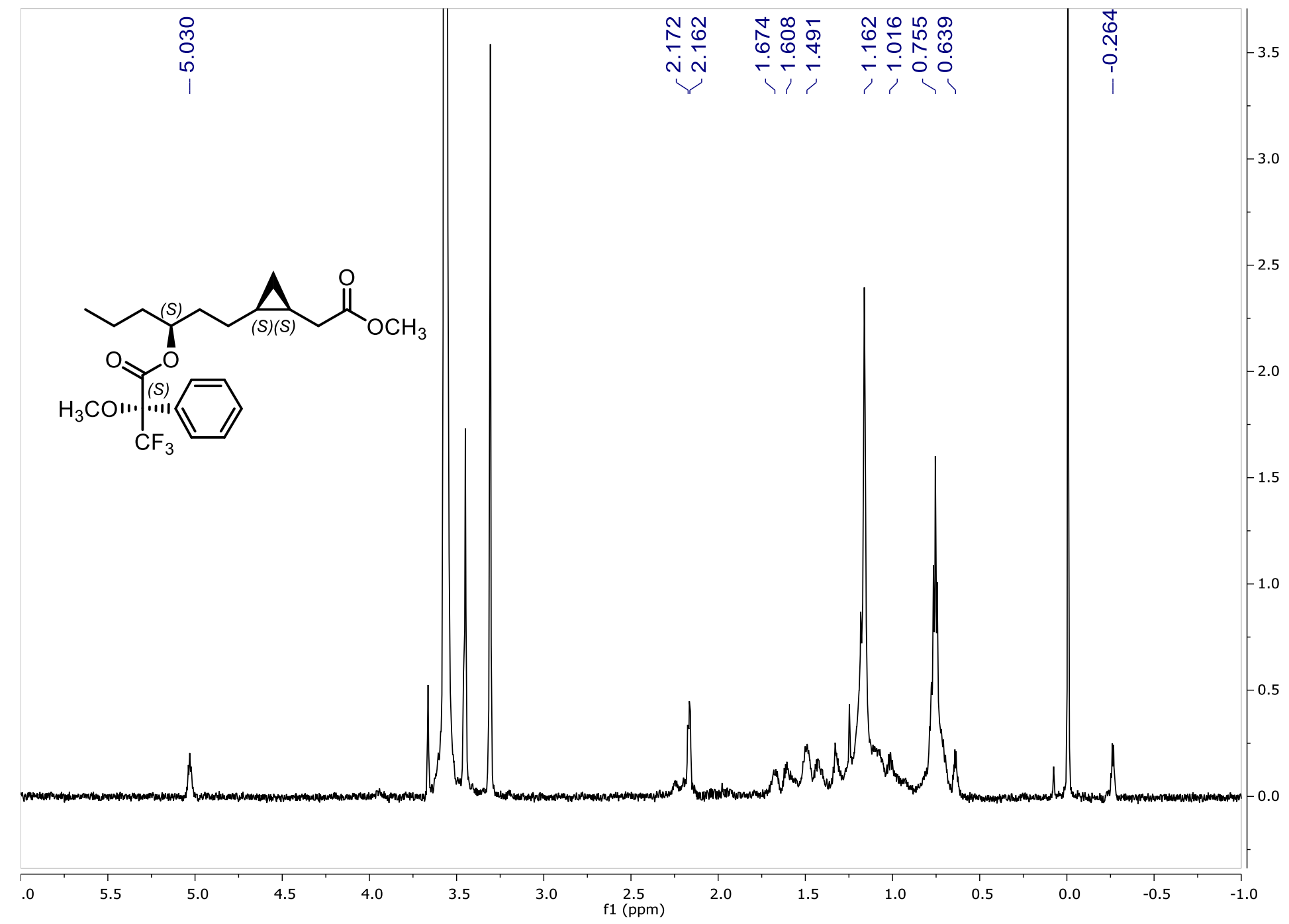


Figure S58a: SELTOCSY (700 MHz, $\left.\mathrm{CDCl}_{3}, 6.5 \% \mathrm{C}_{5} \mathrm{D}_{5} \mathrm{~N}\right)$ of methyl 7-hydroxy-cis-3,4-methylene decanoate (S)-MTPA ester ((S)10).

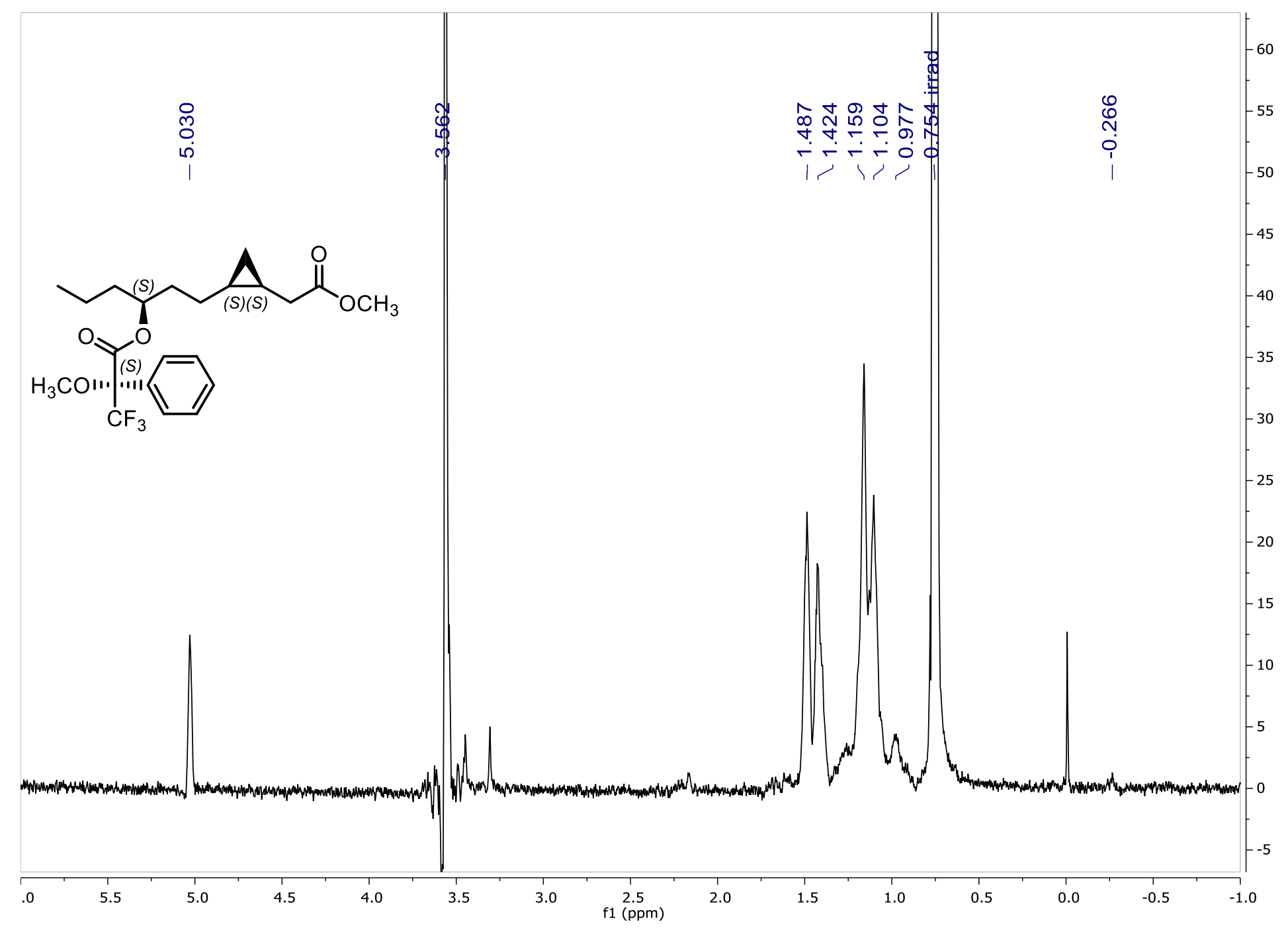


Figure S58b: SELTOCSY (700 MHz, $\left.\mathrm{CDCl}_{3}, 6.5 \% \mathrm{C}_{5} \mathrm{D}_{5} \mathrm{~N}\right)$ of methyl 7-hydroxy-cis-3,4-methylene decanoate (S)-MTPA ester ((S)$10)$.

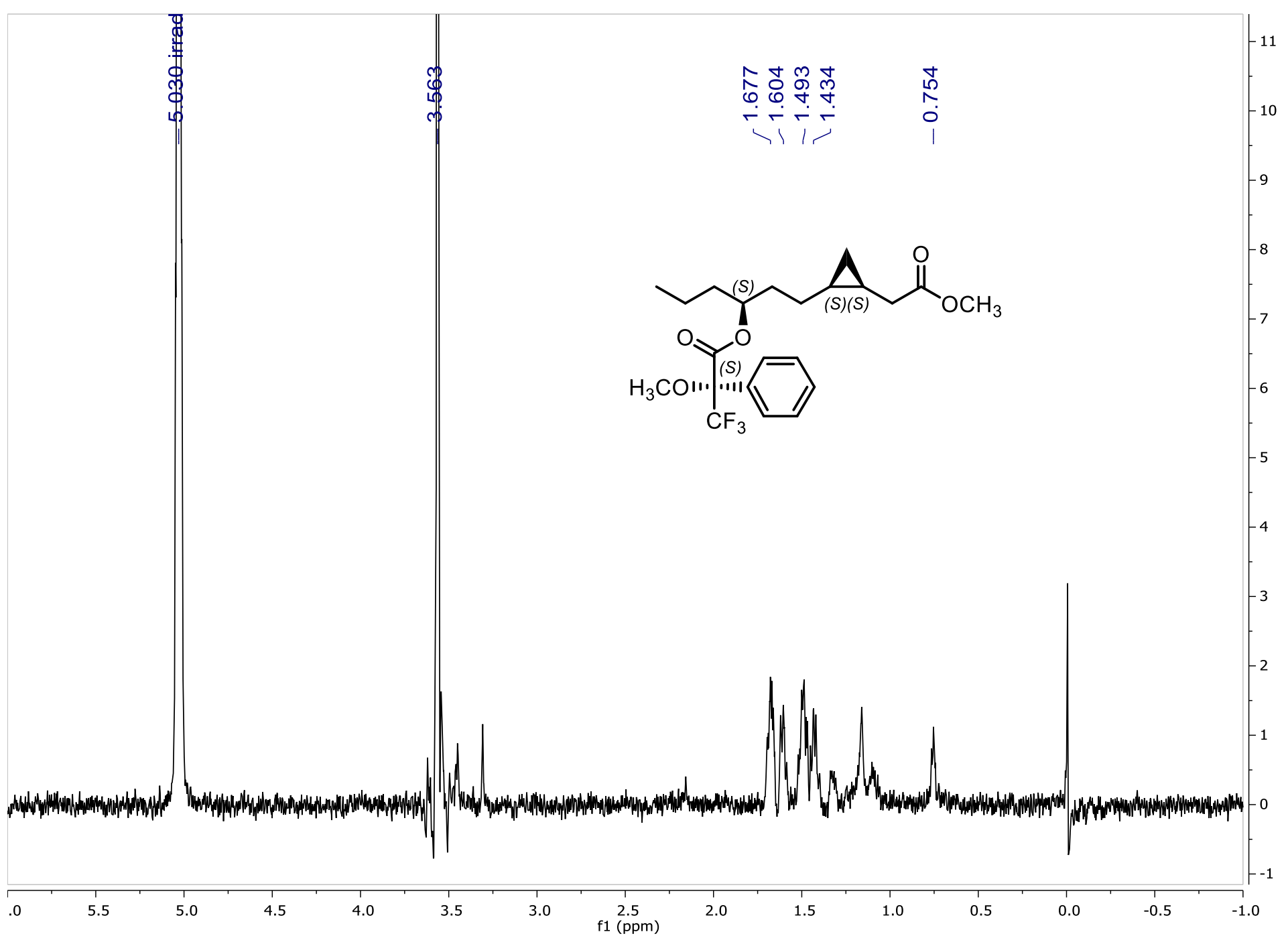


Figure S58c: SELTOCSY (700 MHz, $\left.\mathrm{CDCl}_{3}, 6.5 \% \mathrm{C}_{5} \mathrm{D}_{5} \mathrm{~N}\right)$ of methyl 7-hydroxy-cis-3,4-methylene decanoate (S)-MTPA ester ((S)$10)$.

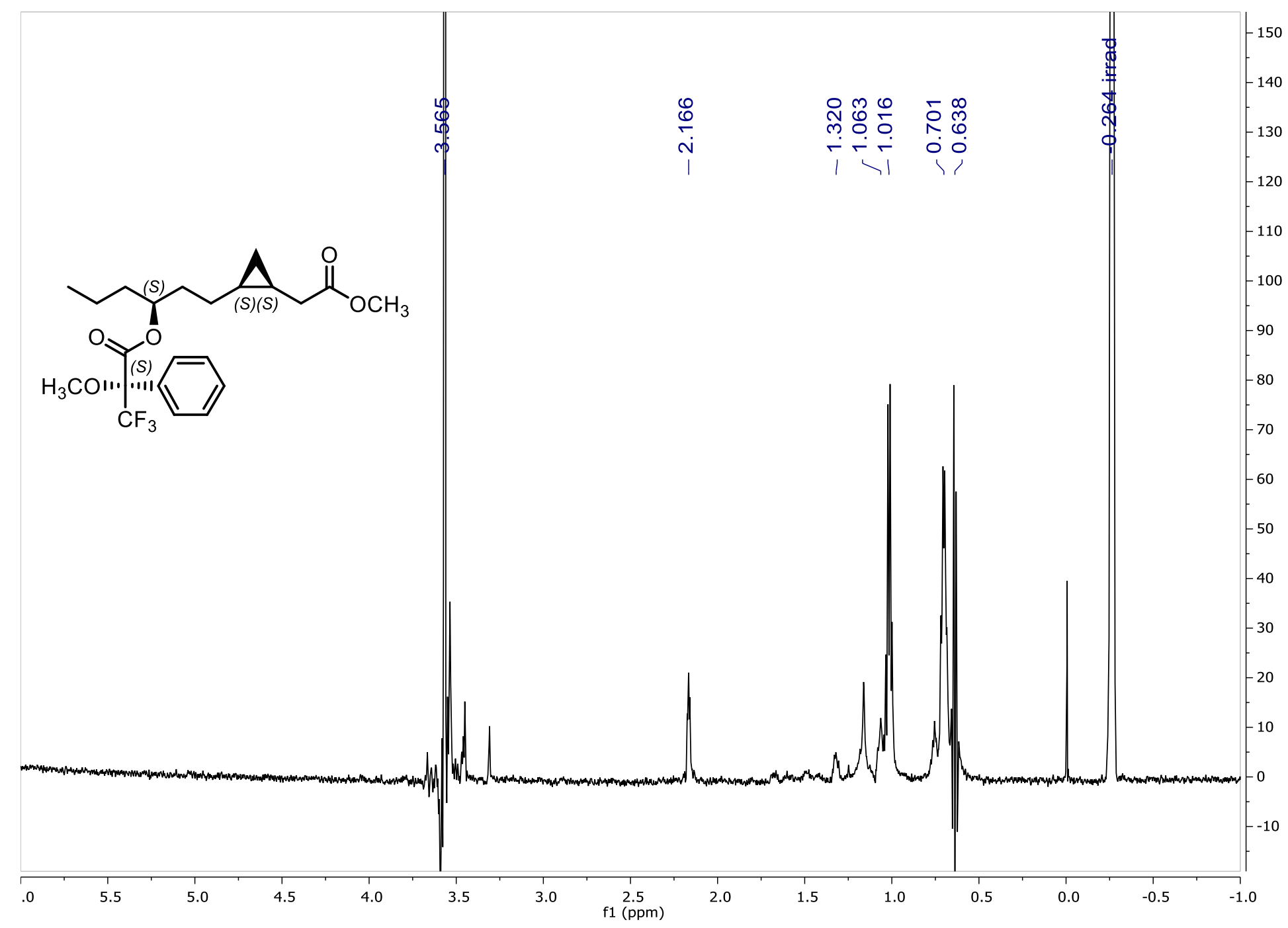


Figure S58d: SELTOCSY $\left(700 \mathrm{MHz}, \mathrm{CDCl}_{3}, 6.5 \% \mathrm{C}_{5} \mathrm{D}_{5} \mathrm{~N}\right)$ of methyl 7-hydroxy-cis-3,4-methylene decanoate (S)-MTPA ester ((S)10).

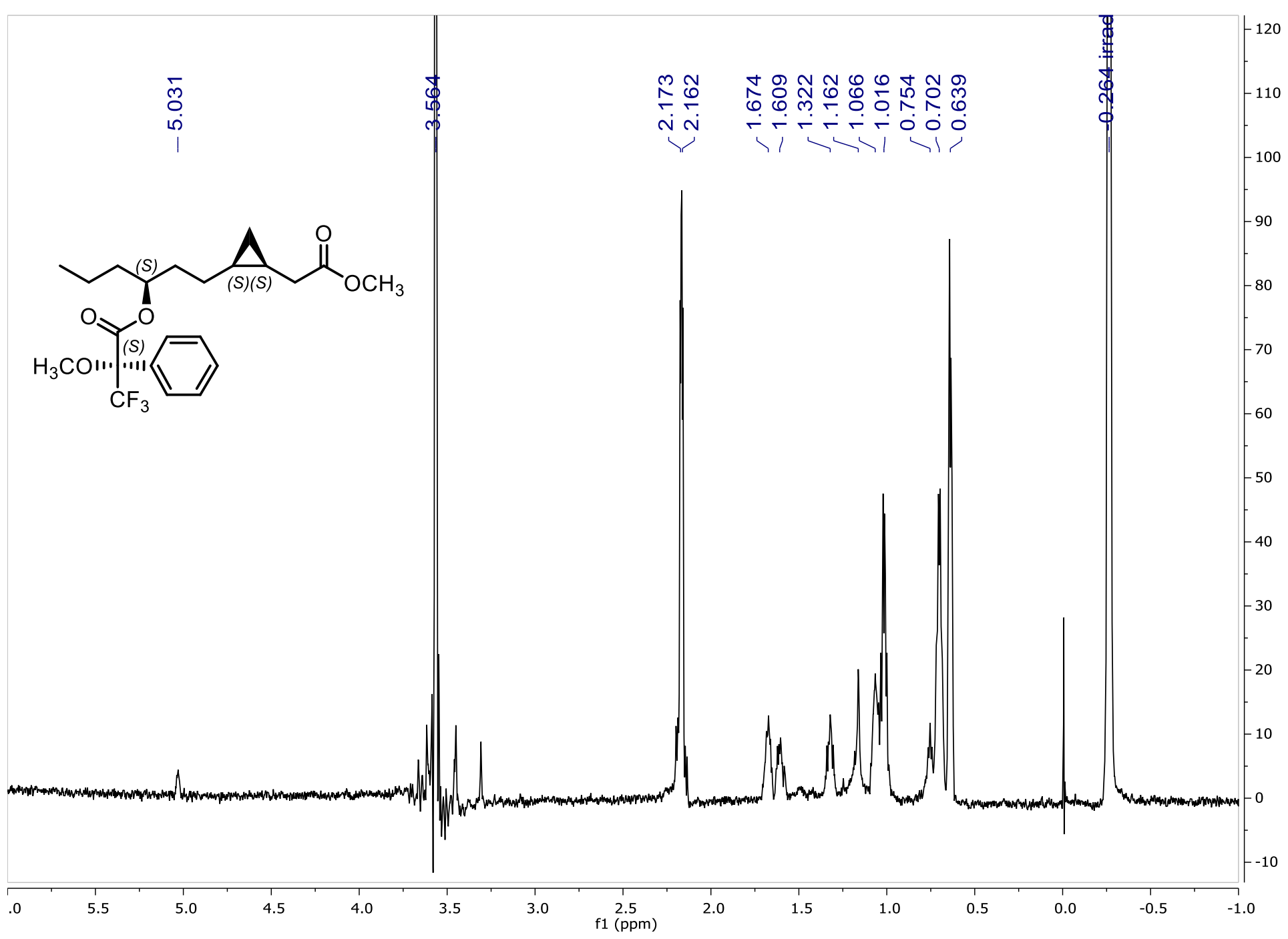


Figure S59: dqf-COSY (700 MHz, $\left.\mathrm{CDCl}_{3}, 6.5 \% \mathrm{C}_{5} \mathrm{D}_{5} \mathrm{~N}\right)$ of methyl 7-hydroxy-cis-3,4-methylene decanoate (S)-MTPA ester ((S)-10).

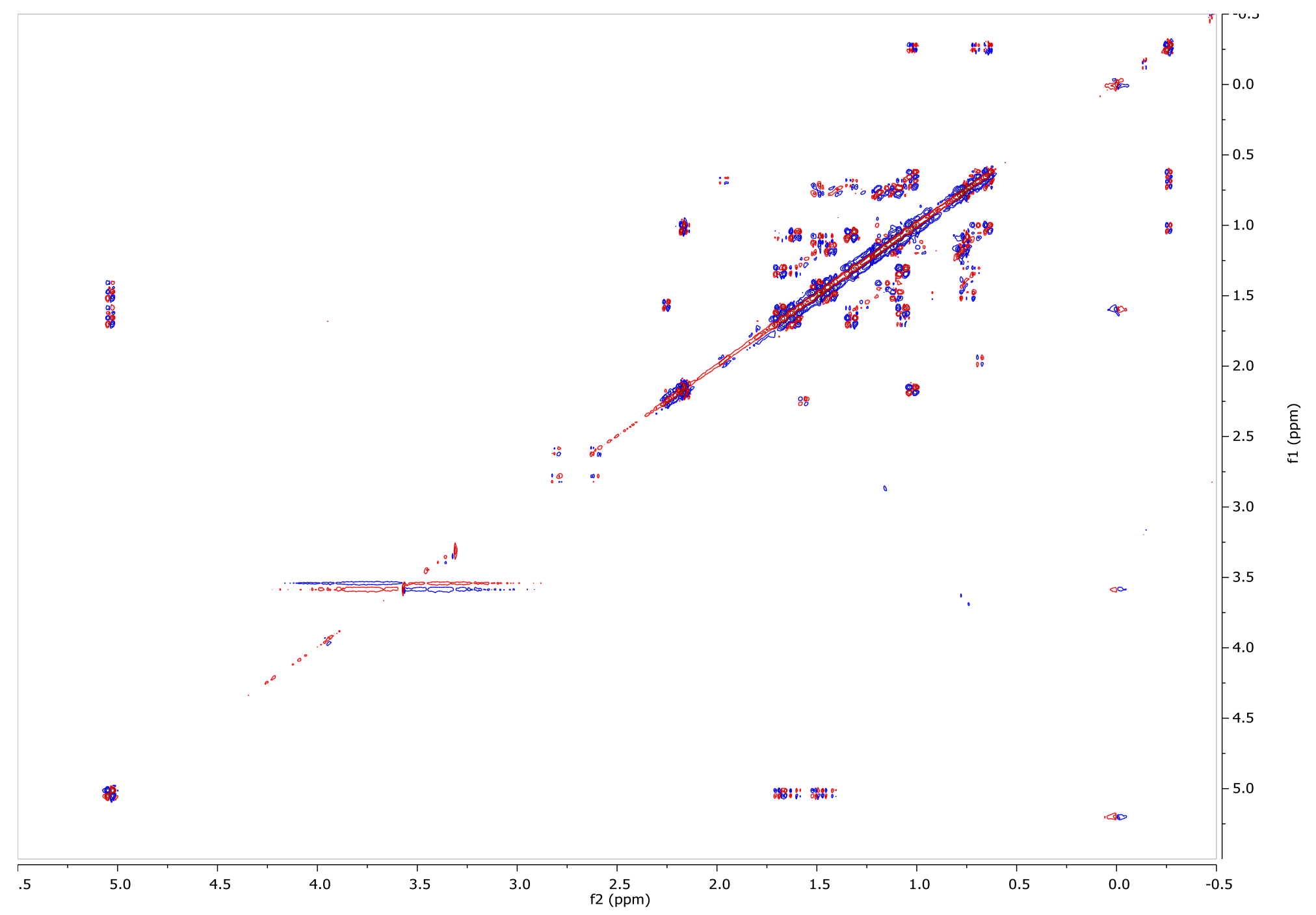


Figure S60: HSQC (700 MHz, $\left.\mathrm{CDCl}_{3}, 6.5 \% \mathrm{C}_{5} \mathrm{D}_{5} \mathrm{~N}\right)$ of methyl 7-hydroxy-cis-3,4-methylene decanoate (S)-MTPA ester ((S)-10).

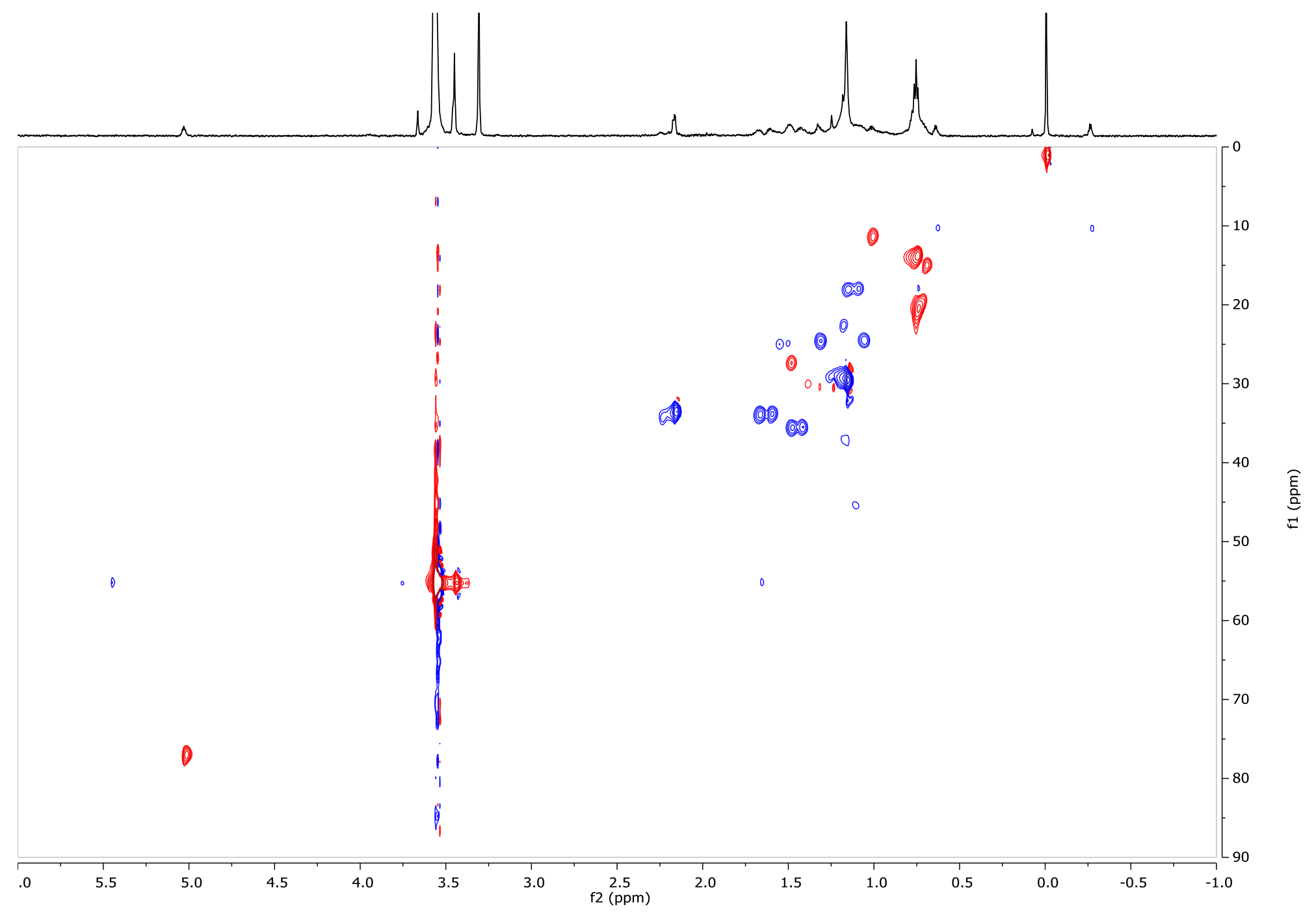


Figure S61: ${ }^{1} \mathrm{H}$ NMR $\left(400 \mathrm{MHz}, \mathrm{CDCl}_{3}\right)$ of 5-O-tert-butyldimethylsilyloxy-pent-2-yn-1-ol.

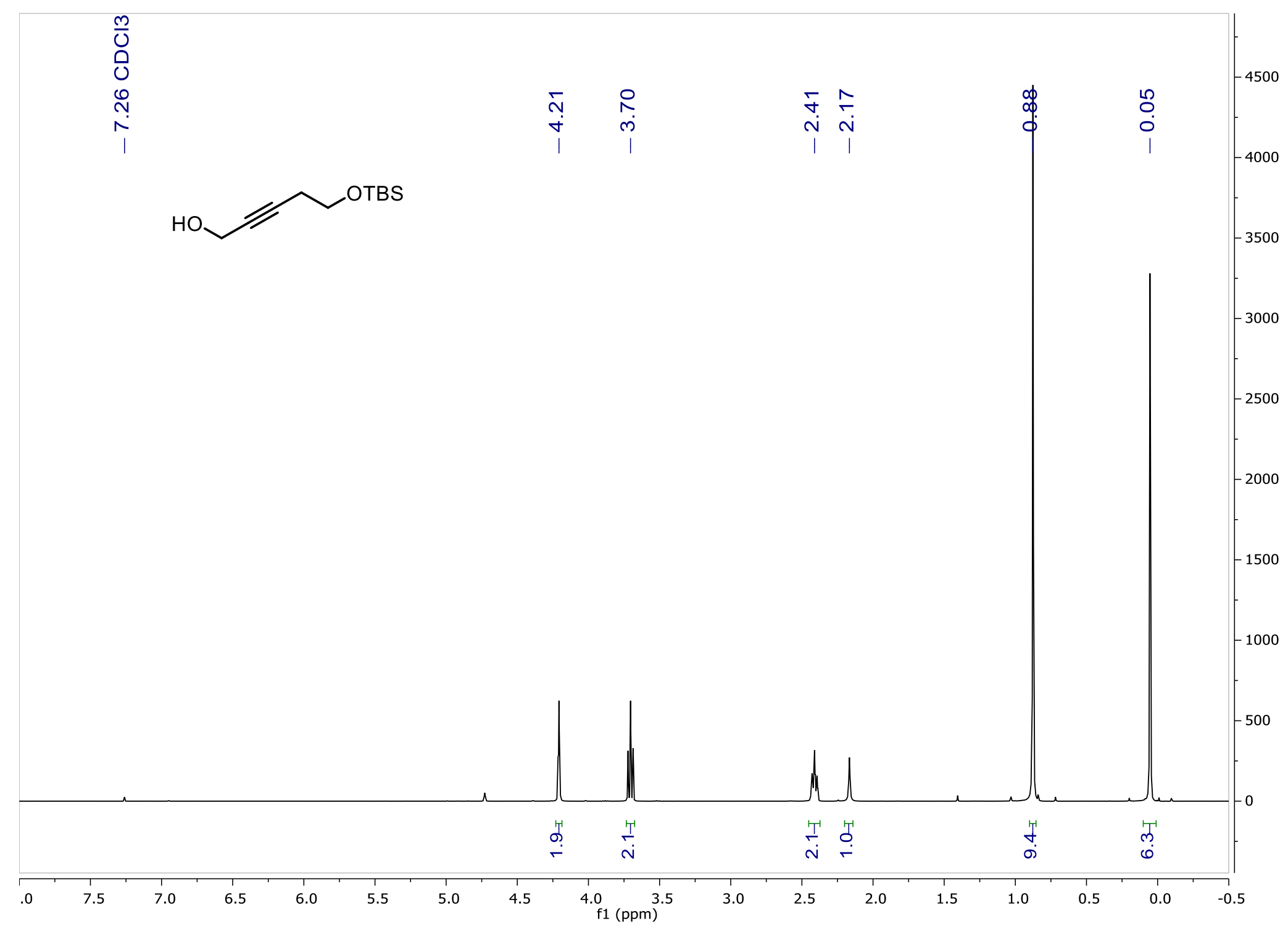


Figure S62: ${ }^{13} \mathrm{C}$ NMR $\left(100 \mathrm{MHz}, \mathrm{CDCl}_{3}\right)$ of 5-O-tert-butyldimethylsilyloxy-pent-2-yn-1-ol.

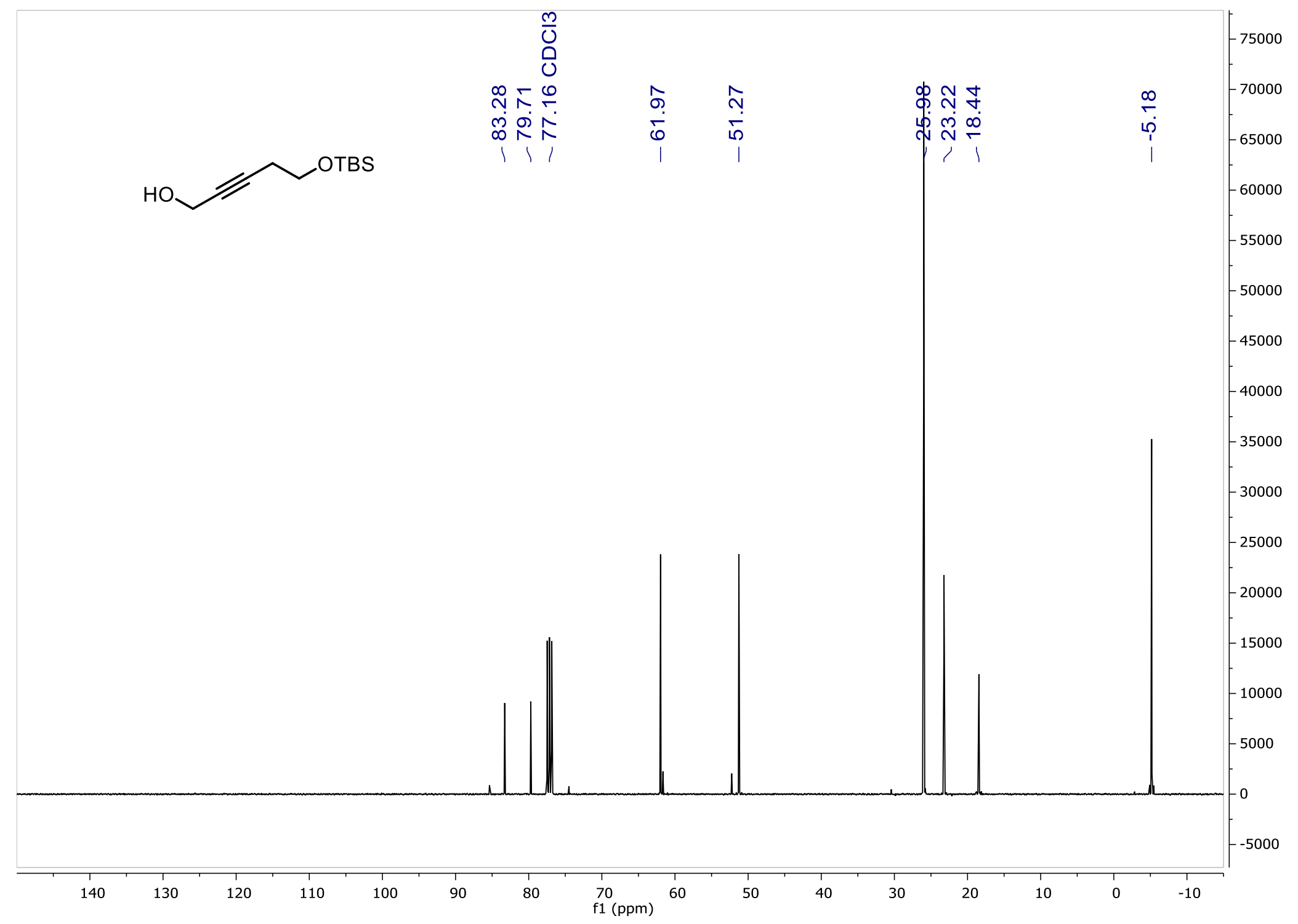


Figure S63: ${ }^{1} \mathrm{H}$ NMR (400 MHz, $\left.\mathrm{CDCl}_{3}\right)$ of (Z)-5-O-tert-butyldimethylsilyloxy-pent-2-en-1-ol (11).

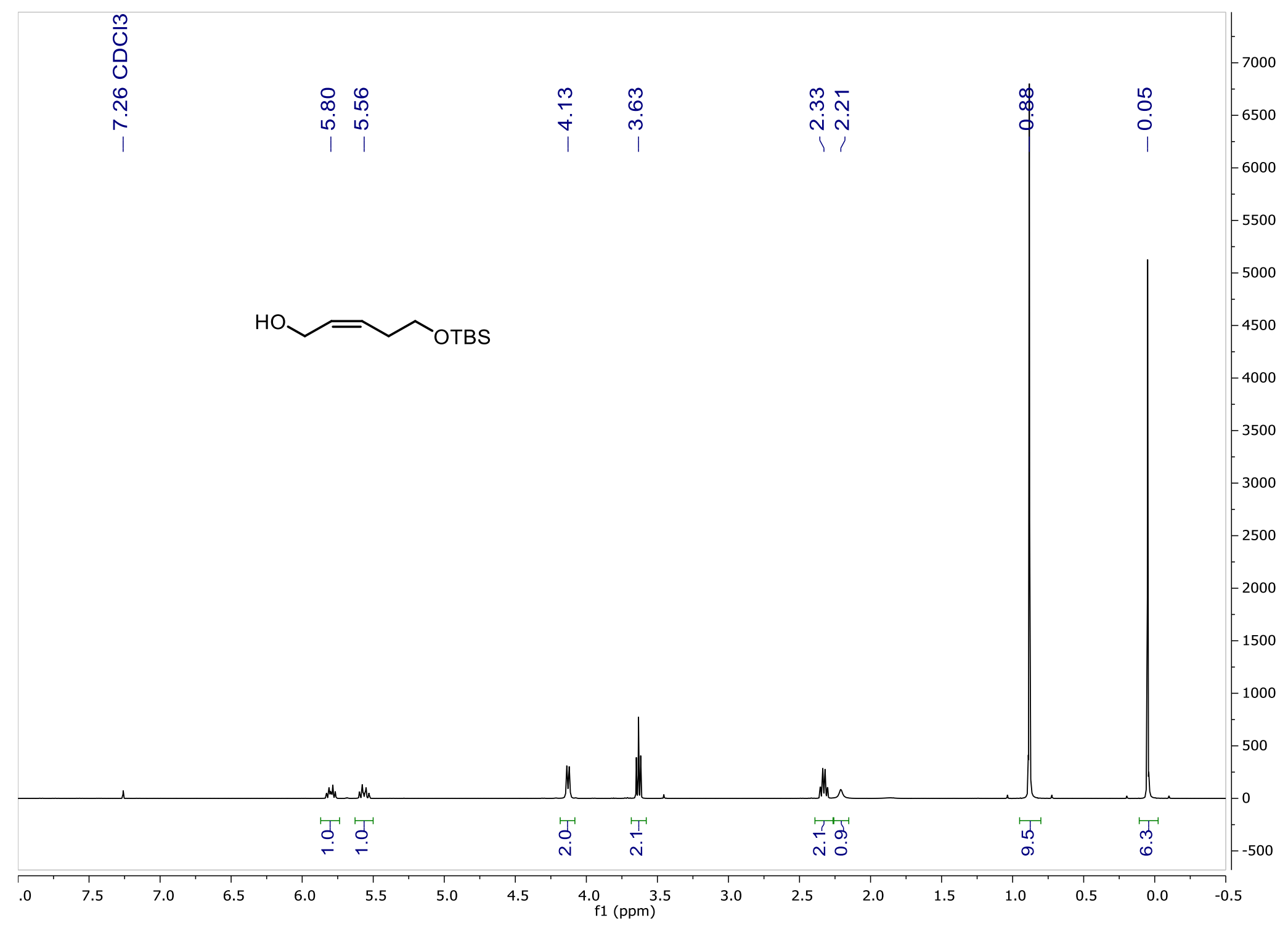


Figure S64: ${ }^{13} \mathrm{C}$ NMR (100 MHz, $\mathrm{CDCl}_{3}$ ) of (Z)-5-O-tert-butyldimethylsilyloxy-pent-2-en-1-ol (11).

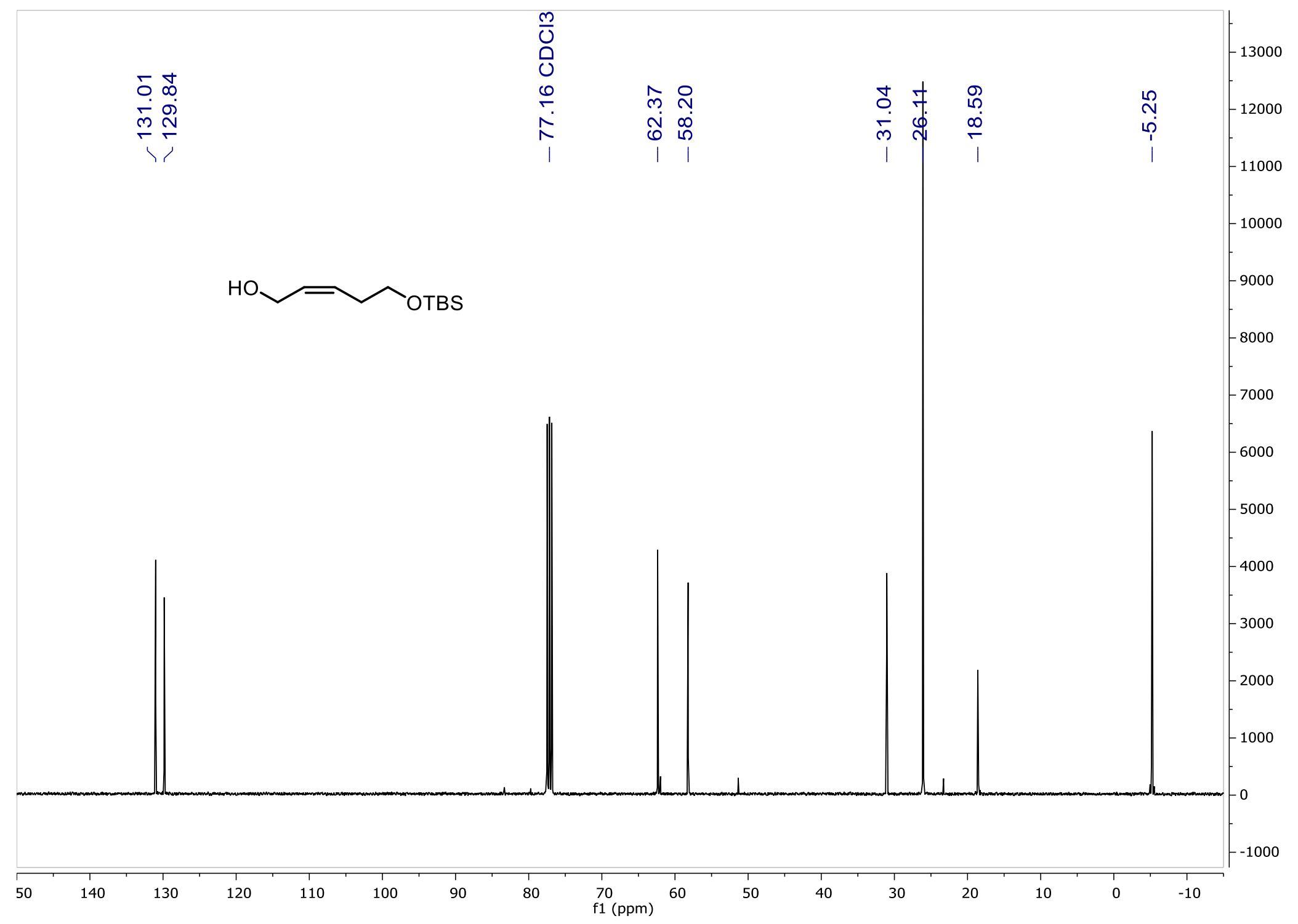


Figure S65: ${ }^{1} \mathrm{H}$ NMR $\left(400 \mathrm{MHz}, \mathrm{CDCl}_{3}\right)$ of (-)-(2S,3S)-5-O-tert-butyldimethylsilyloxy-cis-2,3-methylenepentan-1-ol (12).

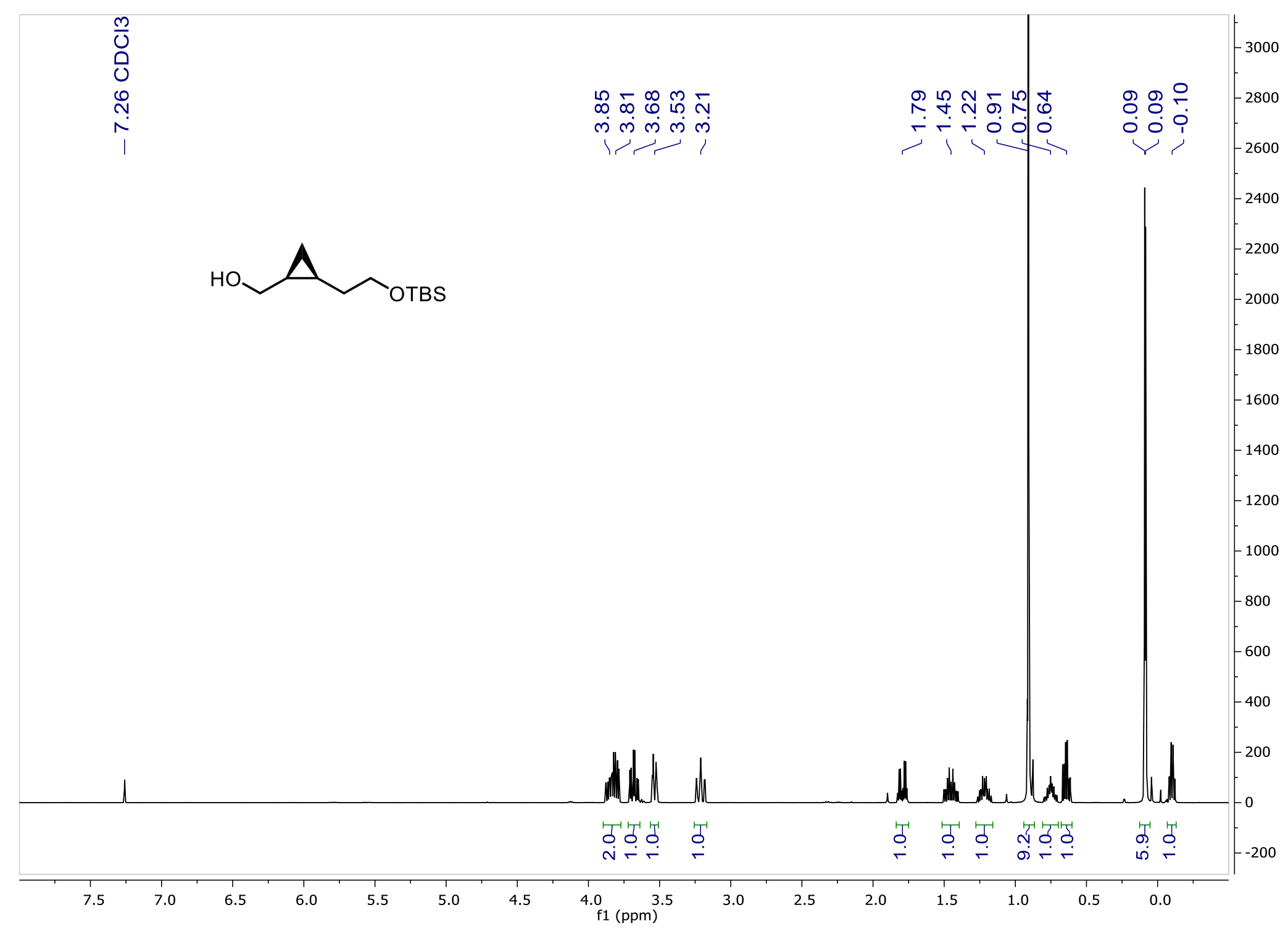


Figure S66: ${ }^{13} \mathrm{C}$ NMR (100 MHz, $\left.\mathrm{CDCl}_{3}\right)$ of (-)-(2S,3S)-5-O-tert-butyldimethylsilyloxy-cis-2,3-methylenepentan-1-ol (12).

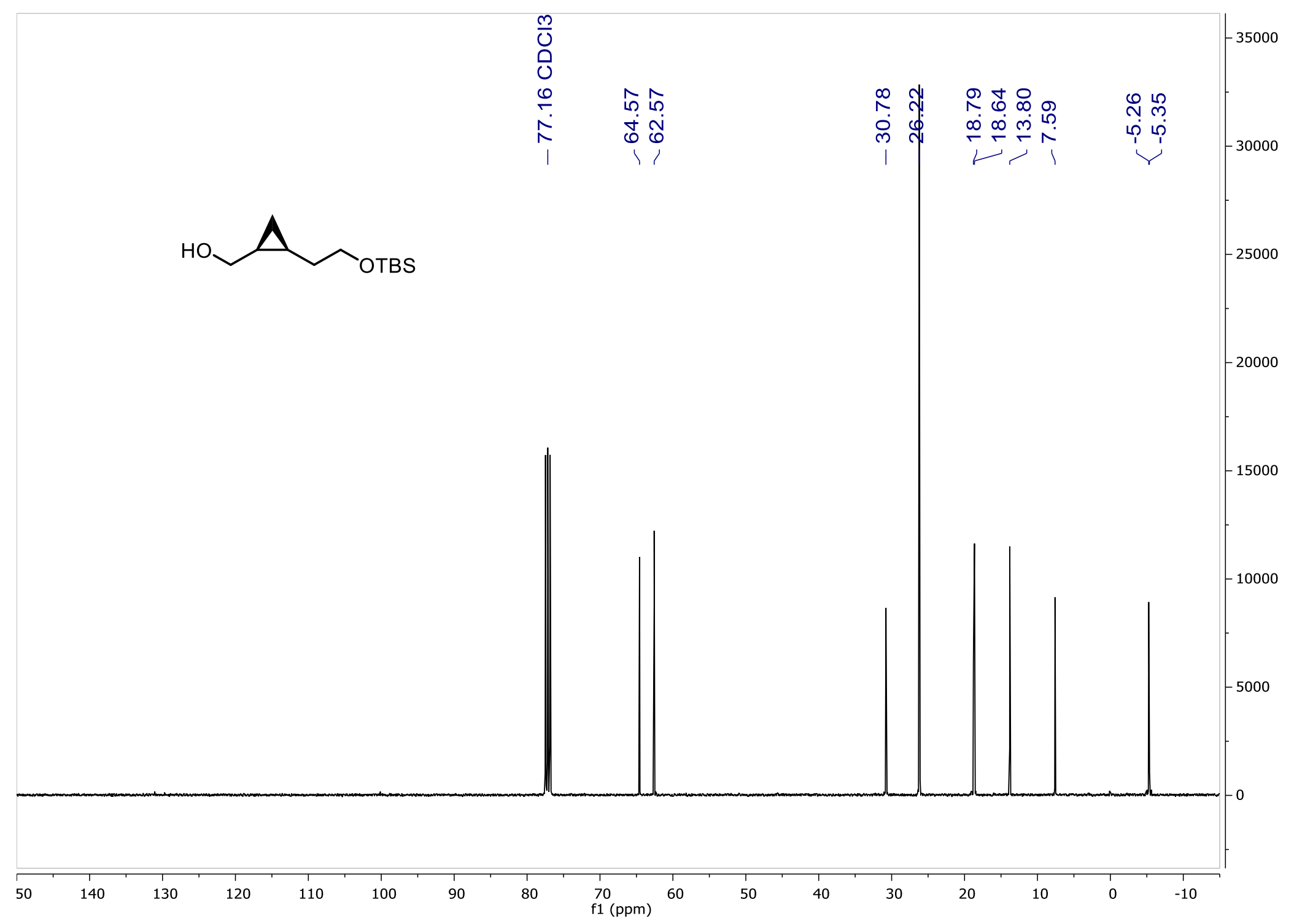


Figure S67: dqf-COSY (400 MHz, $\mathrm{CDCl}_{3}$ ) of (-)-(2S,3S)-5-O-tert-butyldimethylsilyloxy-cis-2,3-methylenepentan-1-ol (12).

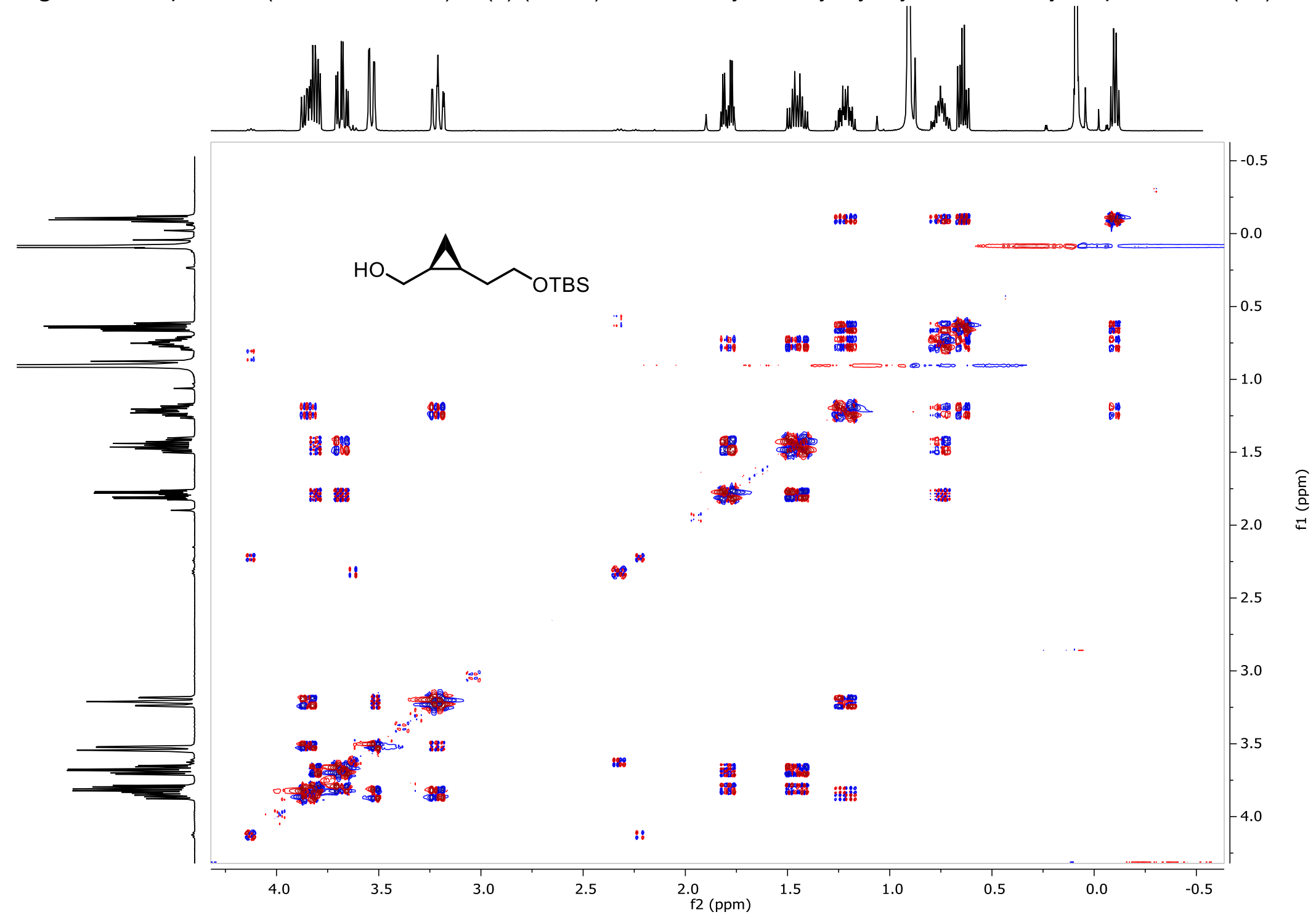


Figure S68: ${ }^{1} \mathrm{H}$ NMR (400 MHz, $\left.\mathrm{CDCl}_{3}\right)$ of (2S,3S)-5-O-tert-butyldimethylsilyloxy-cis-2,3-methylenepentanal (13).

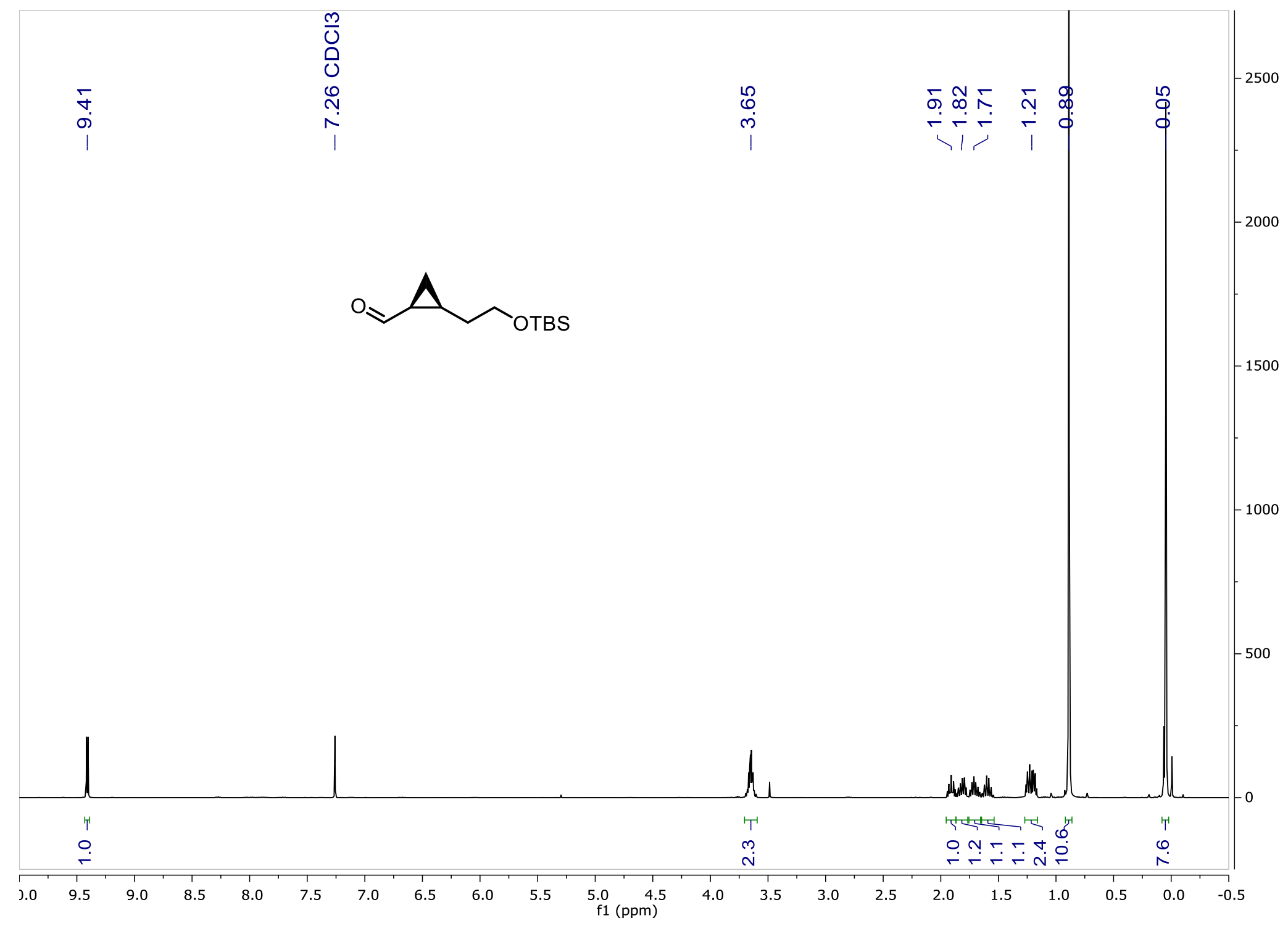


Figure S69: ${ }^{13} \mathrm{C}$ NMR (100 MHz, $\mathrm{CDCl}_{3}$ ) of (2S,3S)-5-O-tert-butyldimethylsilyloxy-cis-2,3-methylenepentanal (13).

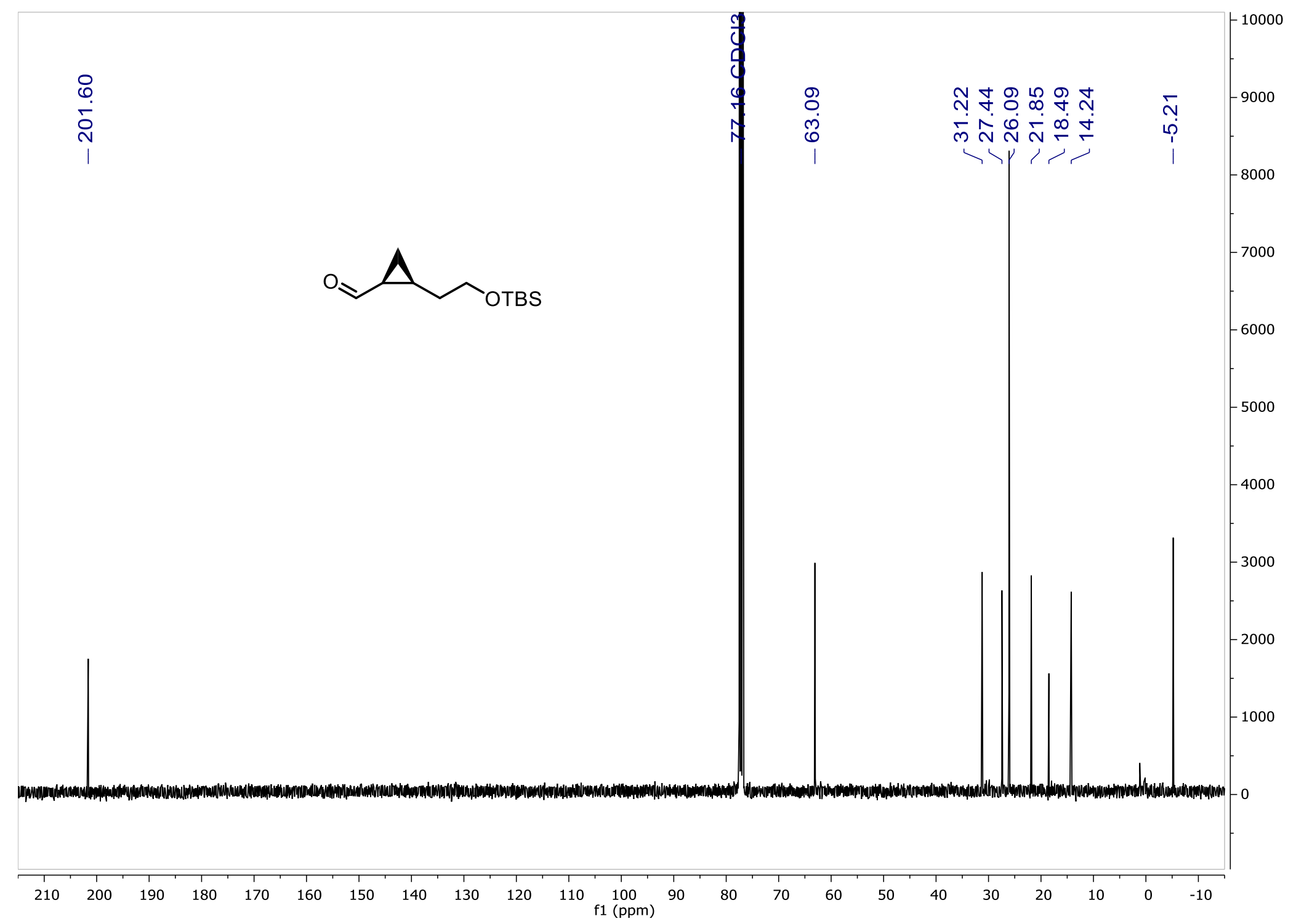


Figure S70: dqf-COSY (400 MHz, $\left.\mathrm{CDCl}_{3}\right)$ of (2S,3S)-5-O-tert-butyldimethylsilyloxy-cis-2,3-methylenepentanal (13).

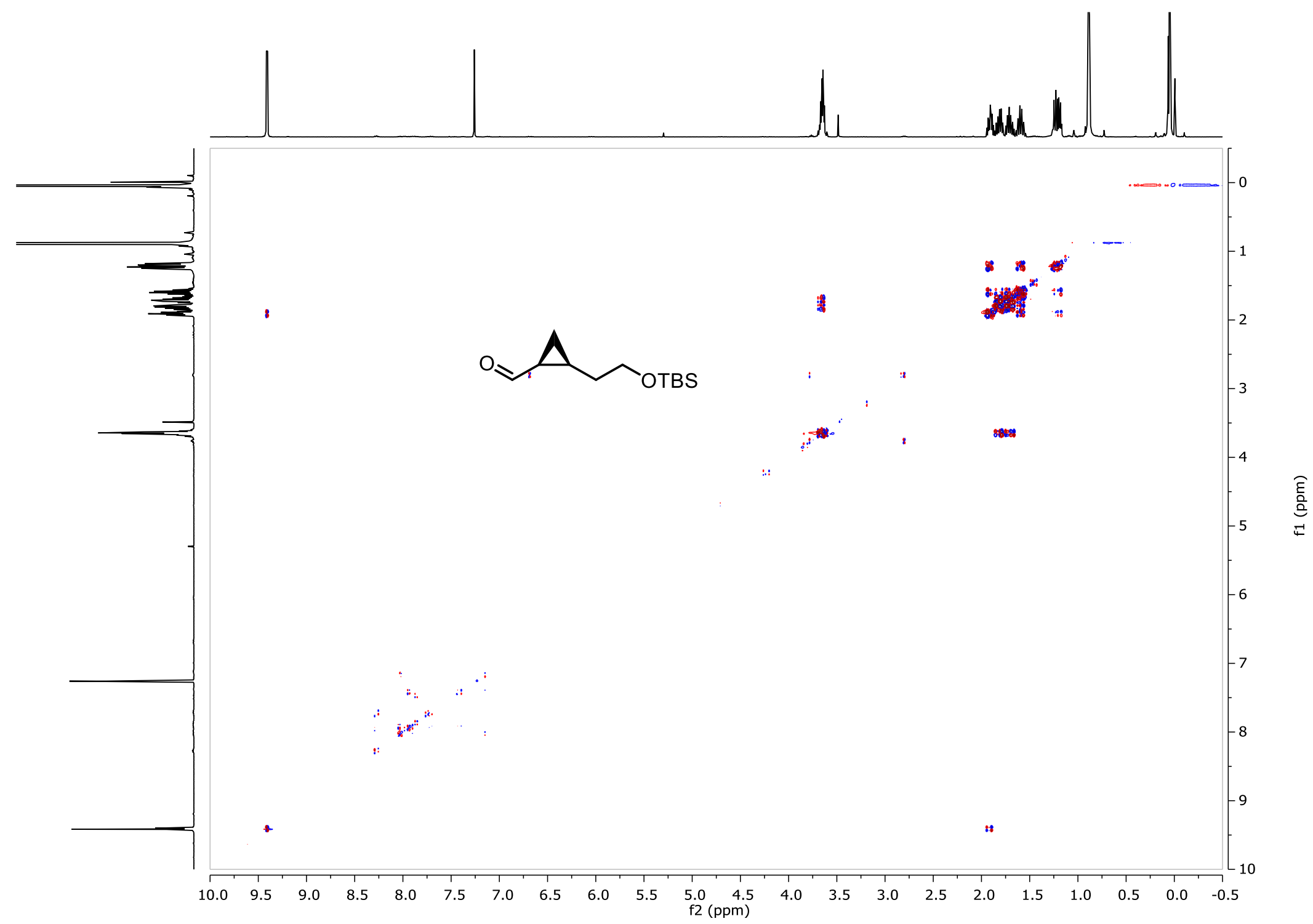


Figure S71: HSQC (400 MHz, $\left.\mathrm{CDCl}_{3}\right)$ of (2S,3S)-5-O-tert-butyldimethylsilyloxy-cis-2,3-methylenepentanal (13).

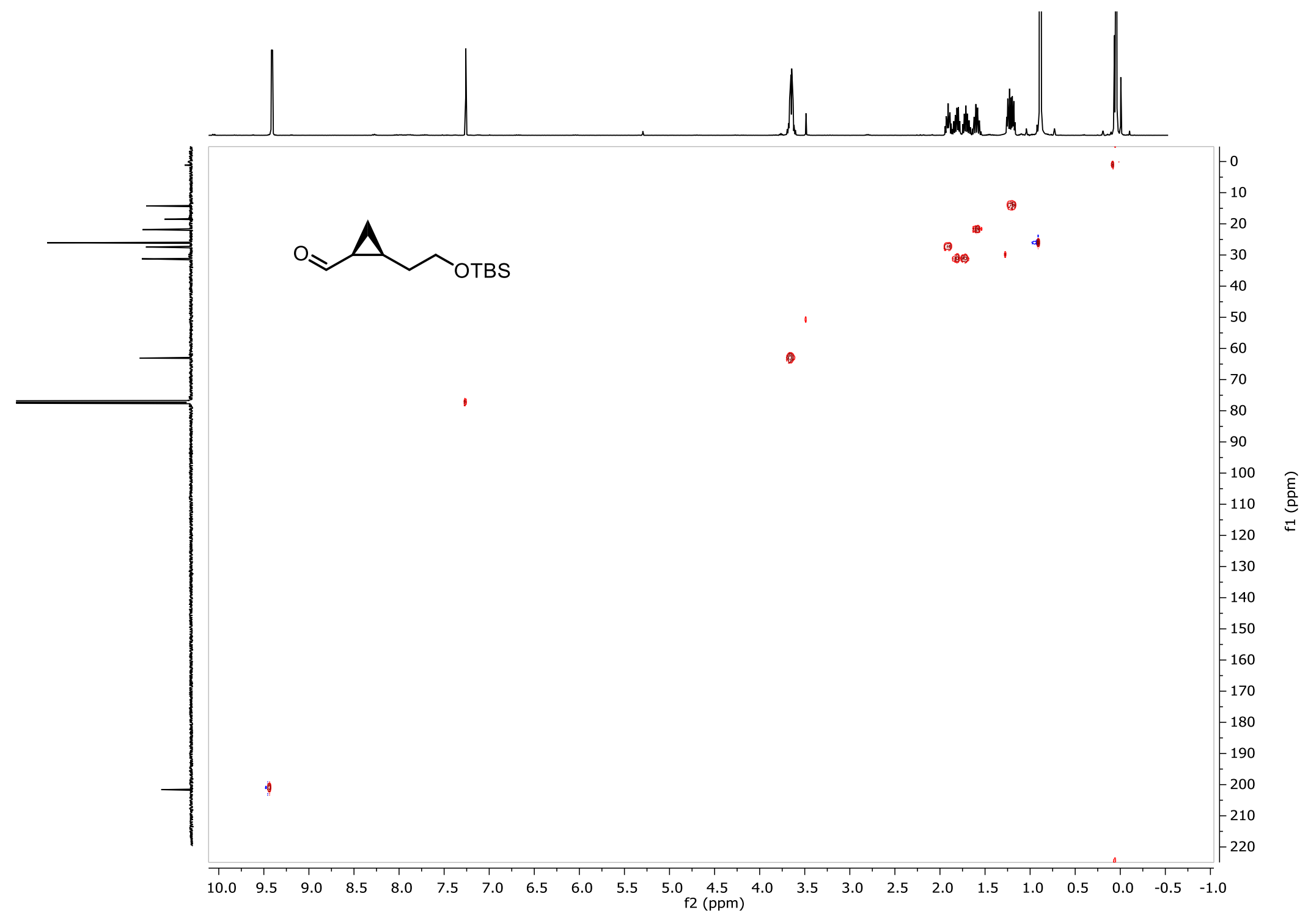


Figure S72: ${ }^{1} \mathrm{H}$ NMR (400 MHz, $\left.\mathrm{CDCl}_{3}\right)$ of (-)-(3R,4S)-6-O-tert-butyldimethylsilyloxy-cis-3,4-methylene-1-hexene (14).

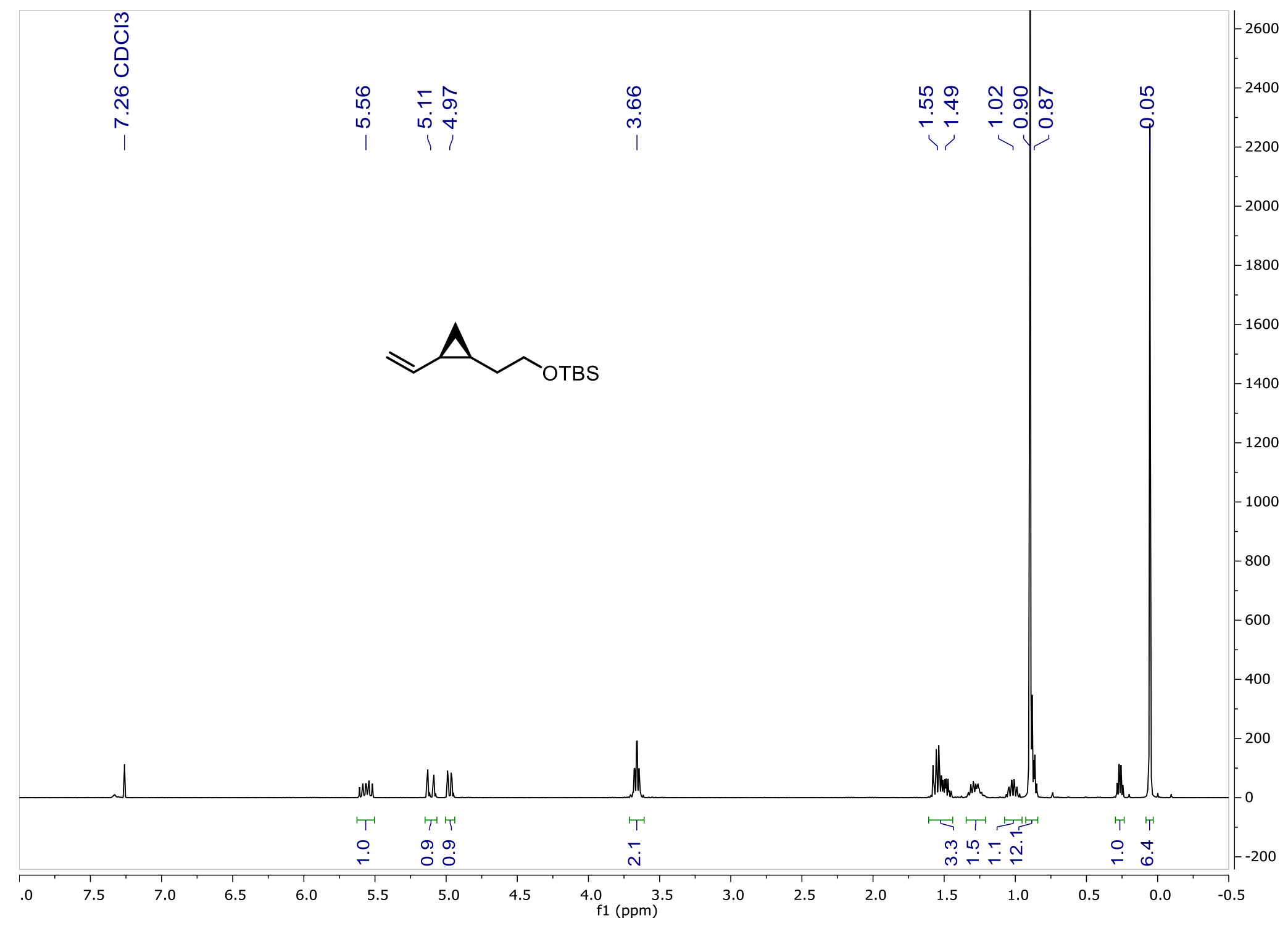


Figure S73: ${ }^{13} \mathrm{C}$ NMR (100 MHz, $\left.\mathrm{CDCl}_{3}\right)$ of (-)-(3R,4S)-6-O-tert-butyldimethylsilyloxy-cis-3,4-methylene-1-hexene (14).

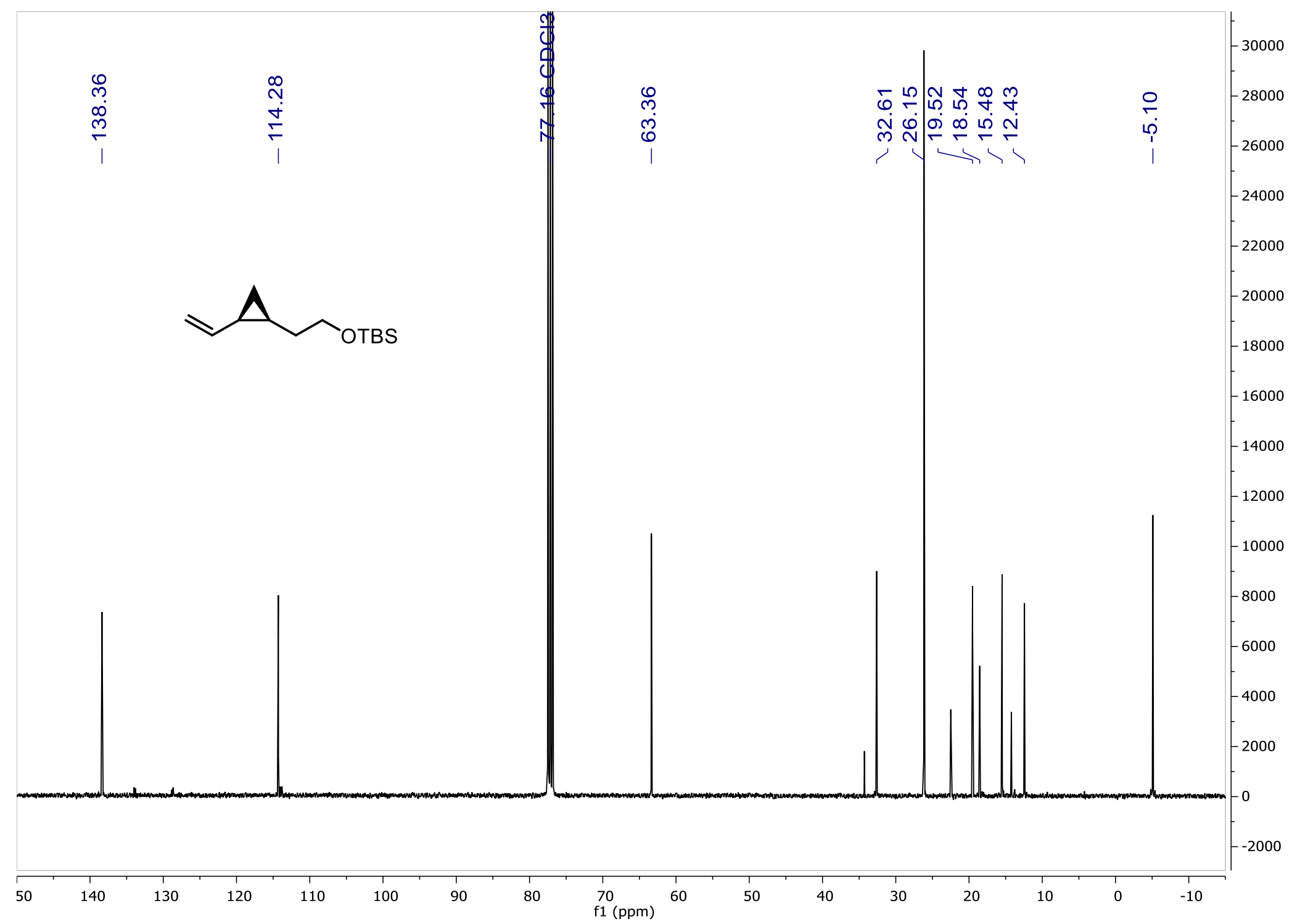


Figure S74: ${ }^{1} \mathrm{H}$ NMR (400 MHz, $\mathrm{CDCl}_{3}$ ) of (-)-(3S)-1-hexen-3-yl acetate (15).

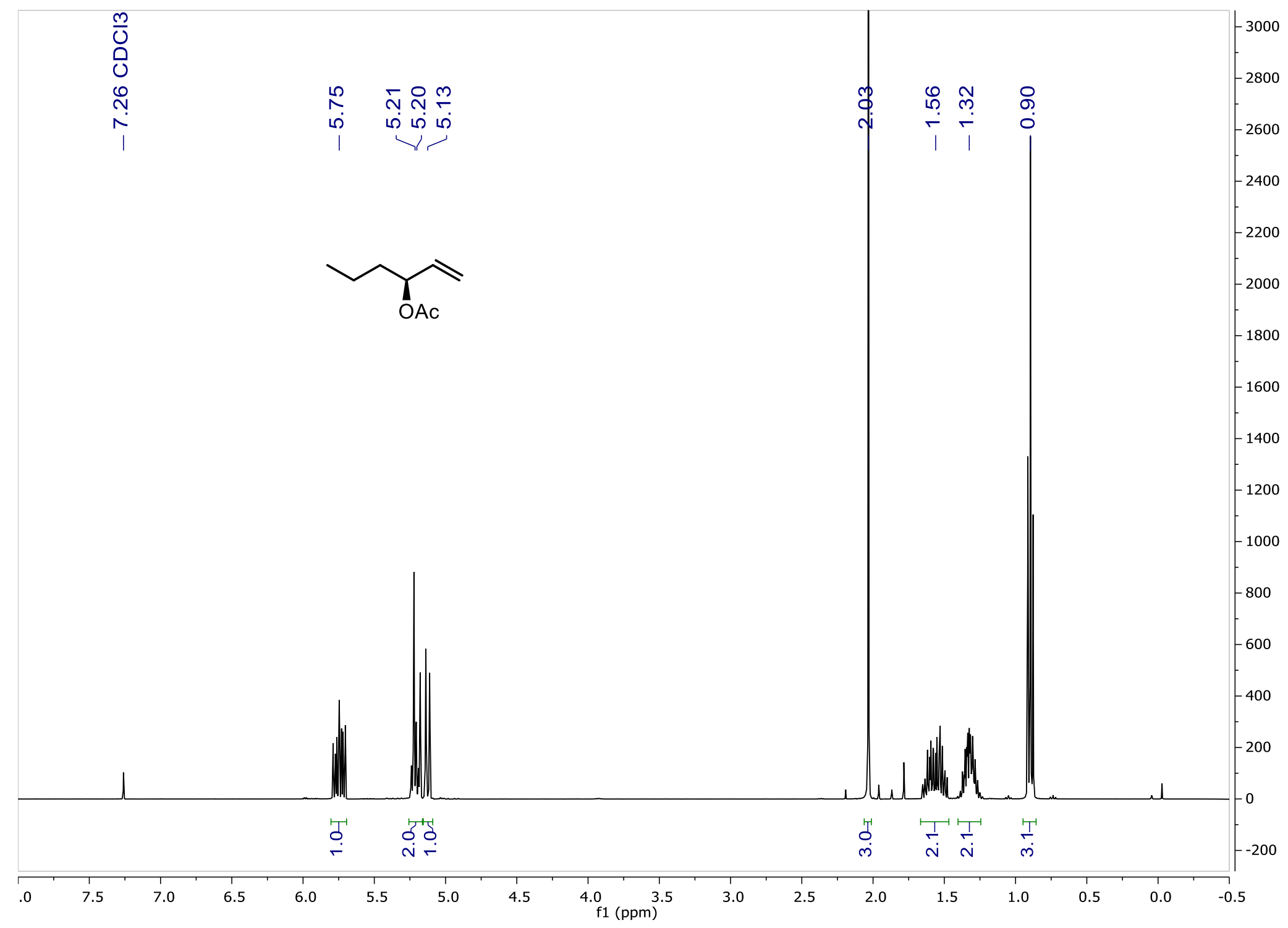


Figure S75: ${ }^{13} \mathrm{C}$ NMR (100 MHz, $\mathrm{CDCl}_{3}$ ) of (-)-(3S)-1-hexen-3-yl acetate (15).

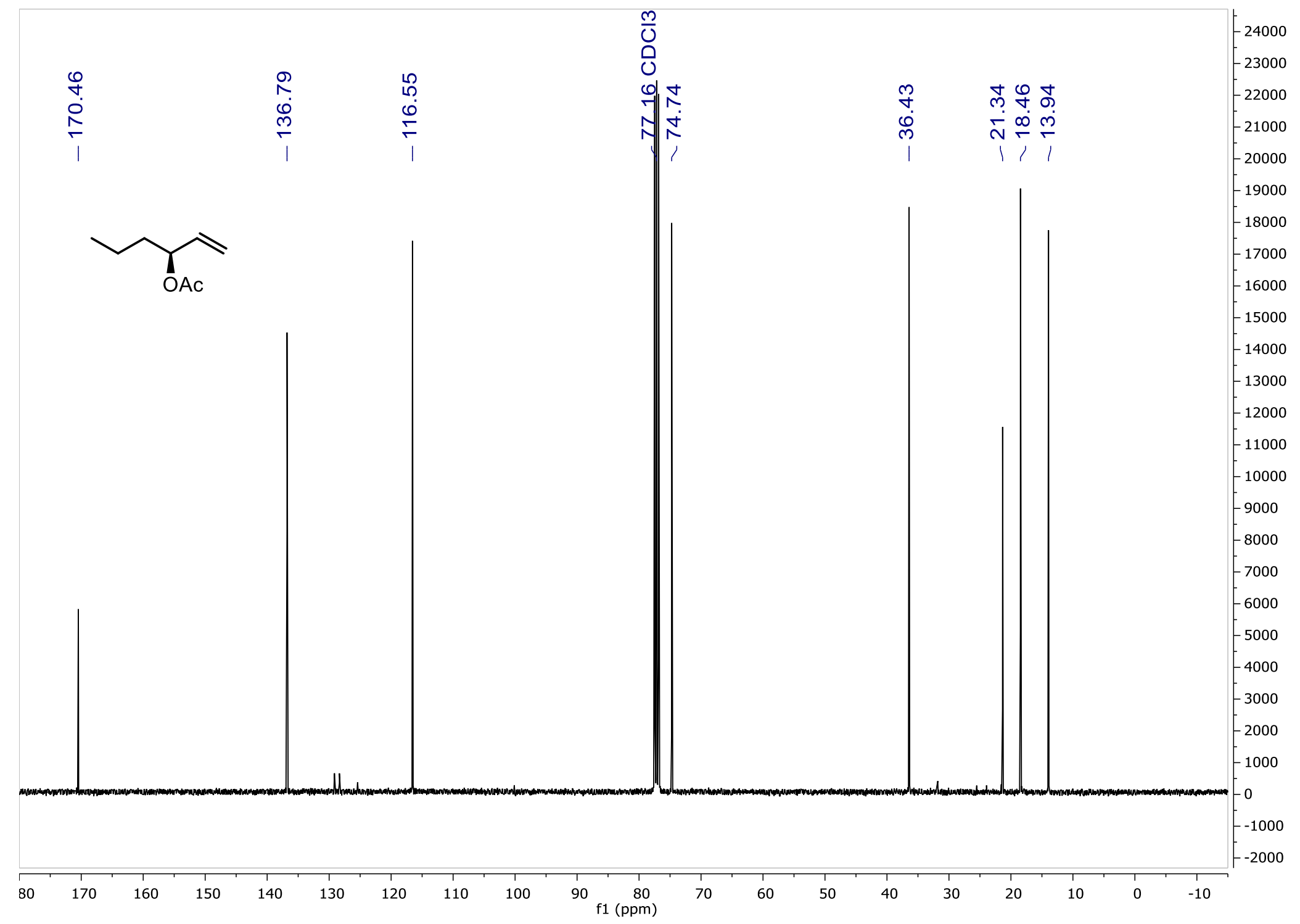


Figure S76: ${ }^{1} \mathrm{H}$ NMR (400 MHz, $\left.\mathrm{CDCl}_{3}\right)$ of $(E)-(3 S, 4 S, 7 S)-1-O-t e r t-b u t y l d i m e t h y l s i l y l o x y-c i s-3,4-m e t h y l e n e d e c-5-e n-7-y l$ acetate (16).

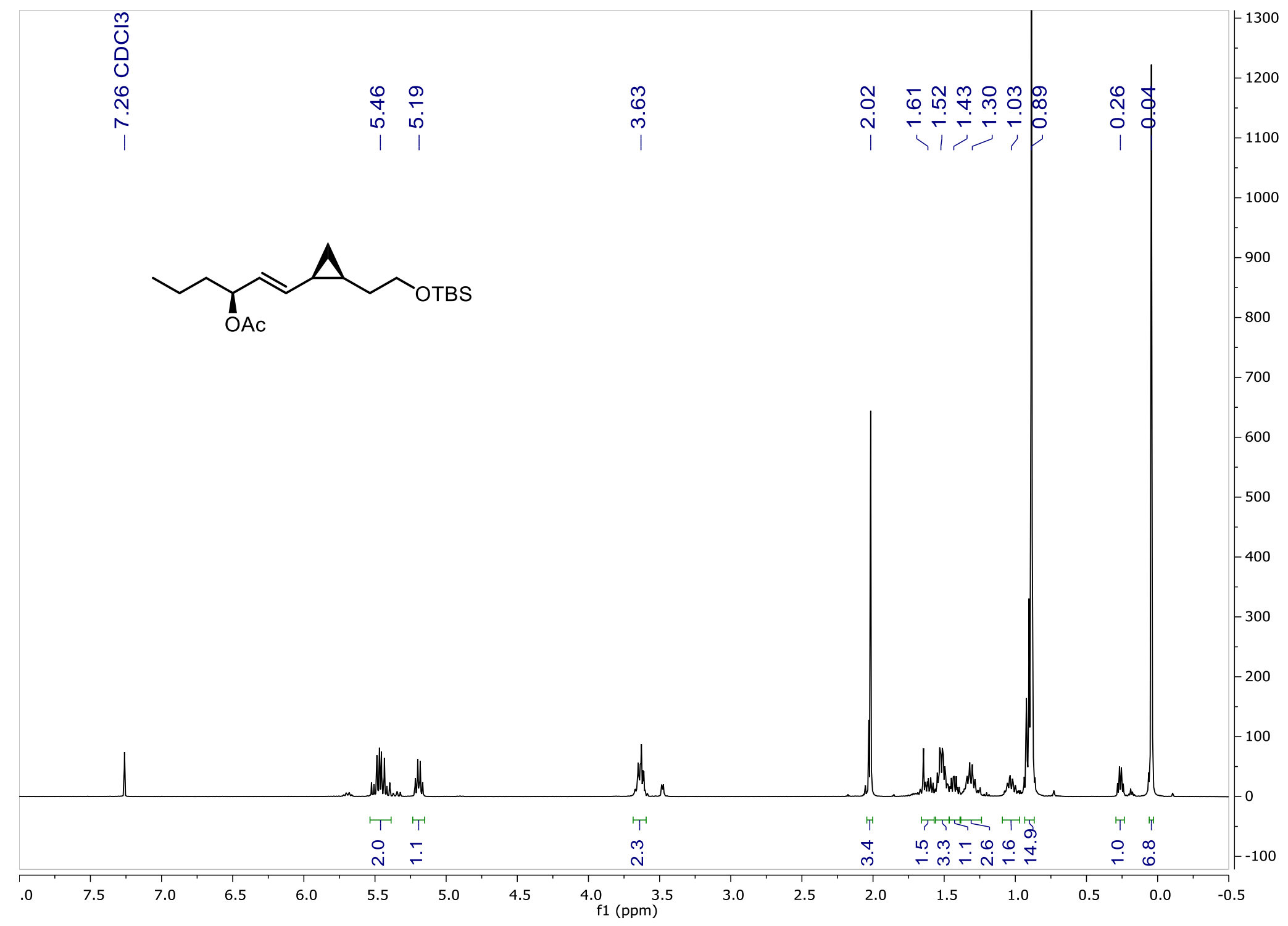


Figure S77: ${ }^{13} \mathrm{C}$ NMR $\left(100 \mathrm{MHz}, \mathrm{CDCl}_{3}\right)$ of $(E)-(3 S, 4 S, 7 S)-1$-O-tert-butyldimethylsilyloxy-cis-3,4-methylenedec-5-en-7-yl acetate (16).

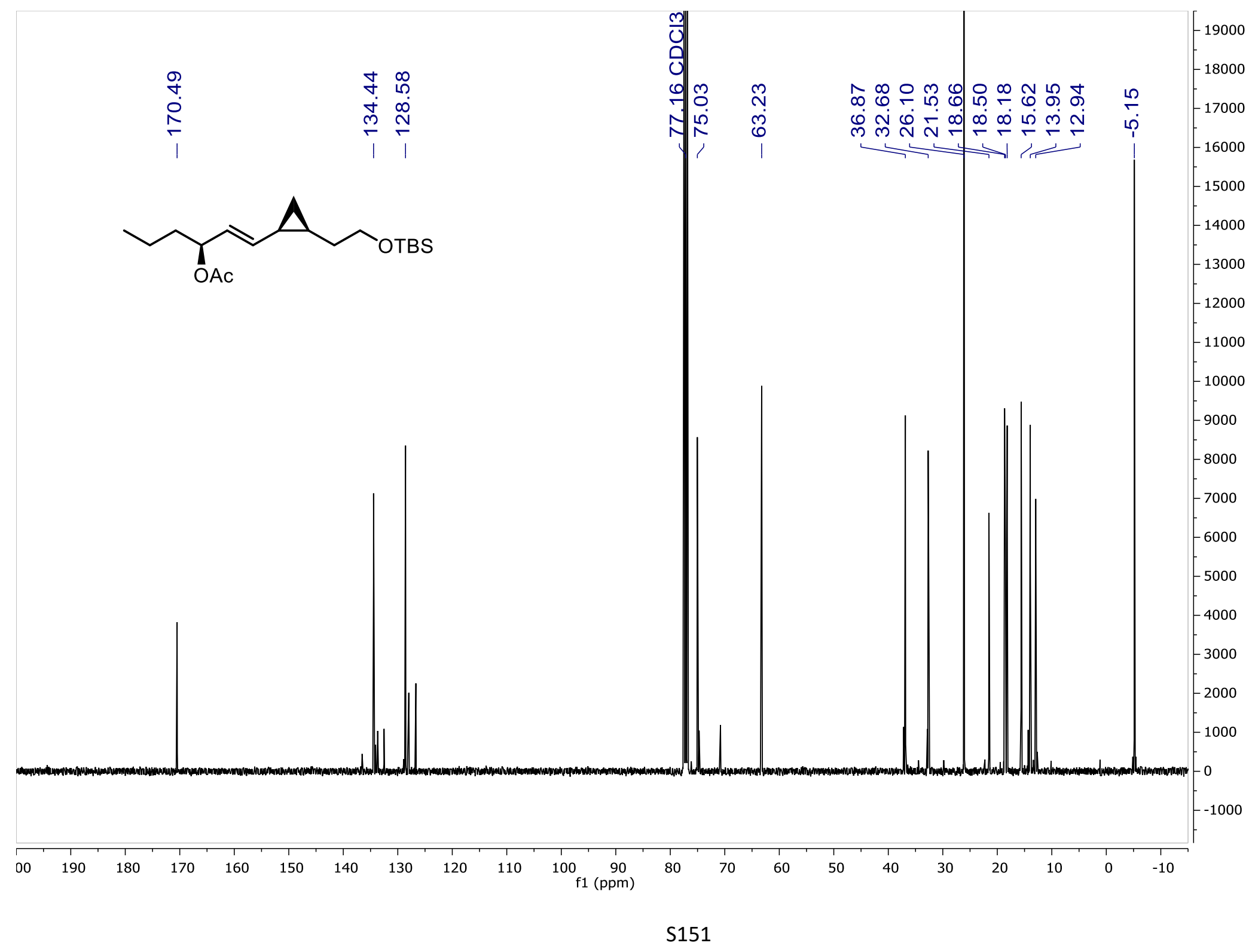


Figure S78: dqf-COSY $\left(400 \mathrm{MHz}_{\mathrm{CDCl}}\right)$ of $(E)-(3 S, 4 S, 7 S)-1-0$-tert-butyldimethylsilyloxy-cis-3,4-methylenedec-5-en-7-yl acetate (16).

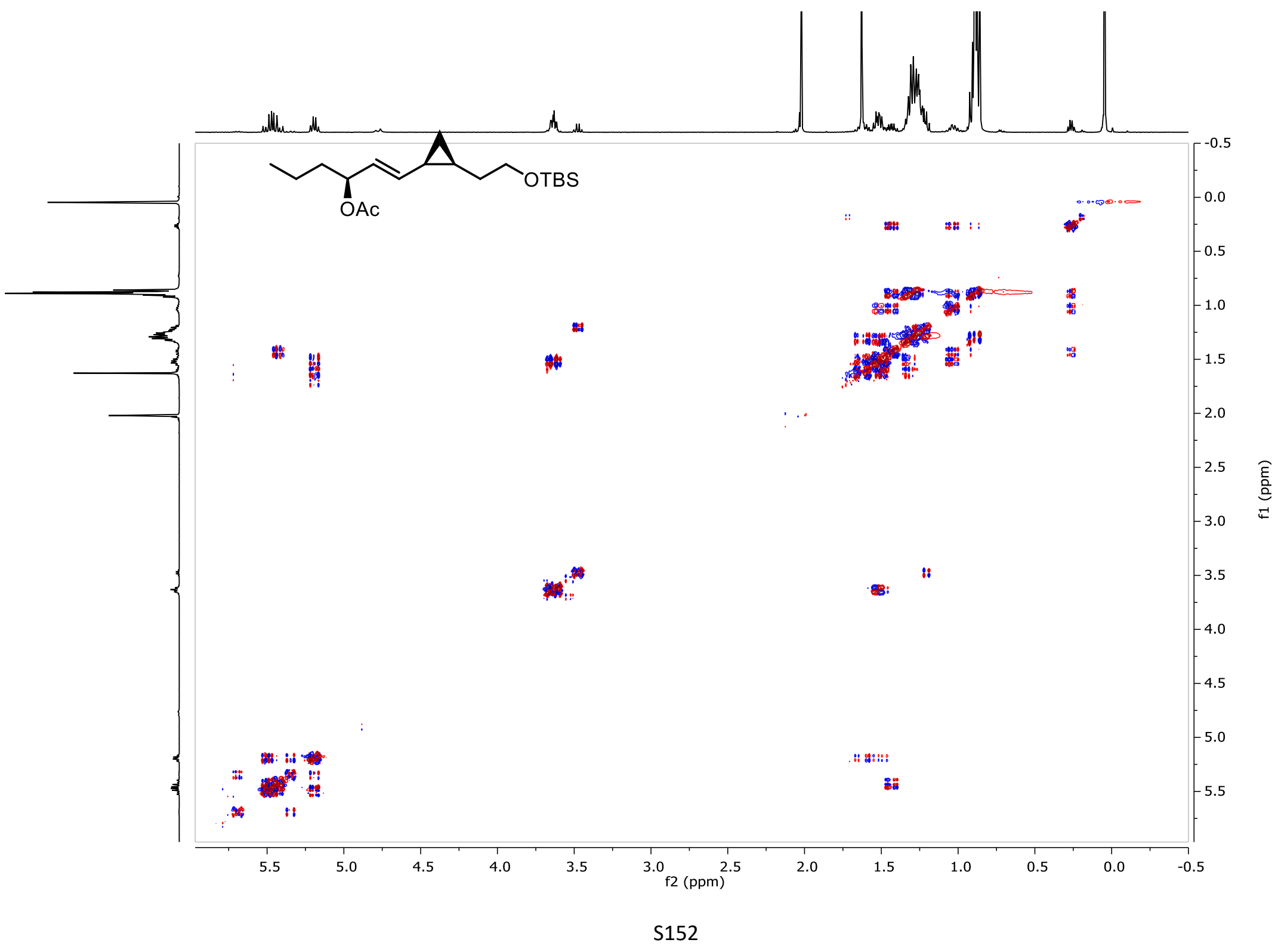


Figure S79: ${ }^{1} \mathrm{H}$ NMR $\left(400 \mathrm{MHz}, \mathrm{CDCl}_{3}\right)$ of $(3 S, 4 S, 7 S)-1-O-t e r t-b u t y l d i m e t h y l s i l y l o x y-c i s-3,4-m e t h y l e n e d e c a n-7-y l$ acetate (17).

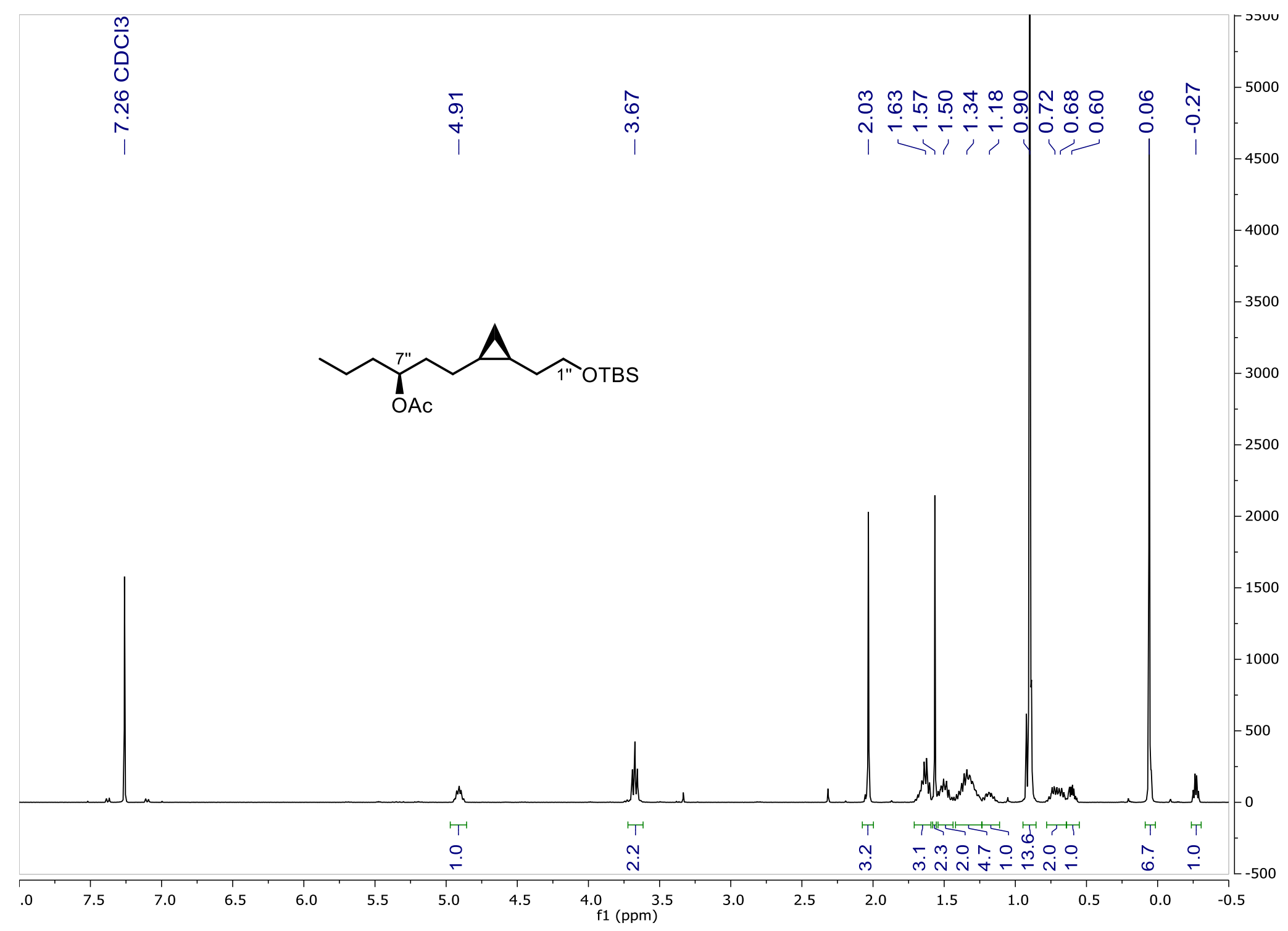


Figure S80: ${ }^{13} \mathrm{C}$ NMR (100 MHz, $\left.\mathrm{CDCl}_{3}\right)$ of (3S,4S,7S)-1-O-tert-butyldimethylsilyloxy-cis-3,4-methylenedecan-7-yl acetate (17).

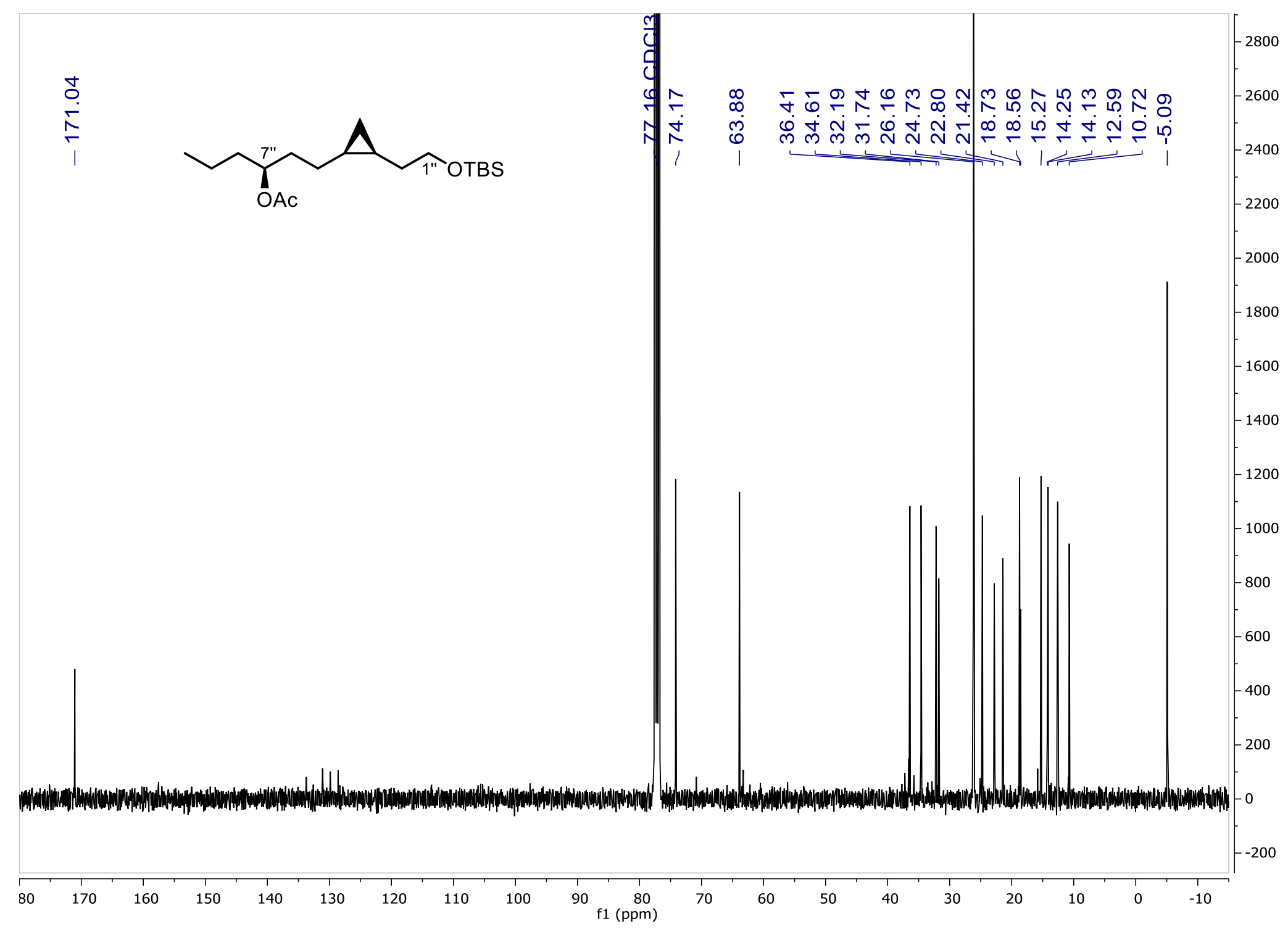


Figure S81: dqf-COSY spectrum (400 MHz, $\left.\mathrm{CDCl}_{3}\right)$ of $(3 S, 4 S, 7 S)-1-O-t e r t-b u t y l d i m e t h y l s i l y l o x y-c i s-3,4-$ methylenedecan-7-yl acetate (17).

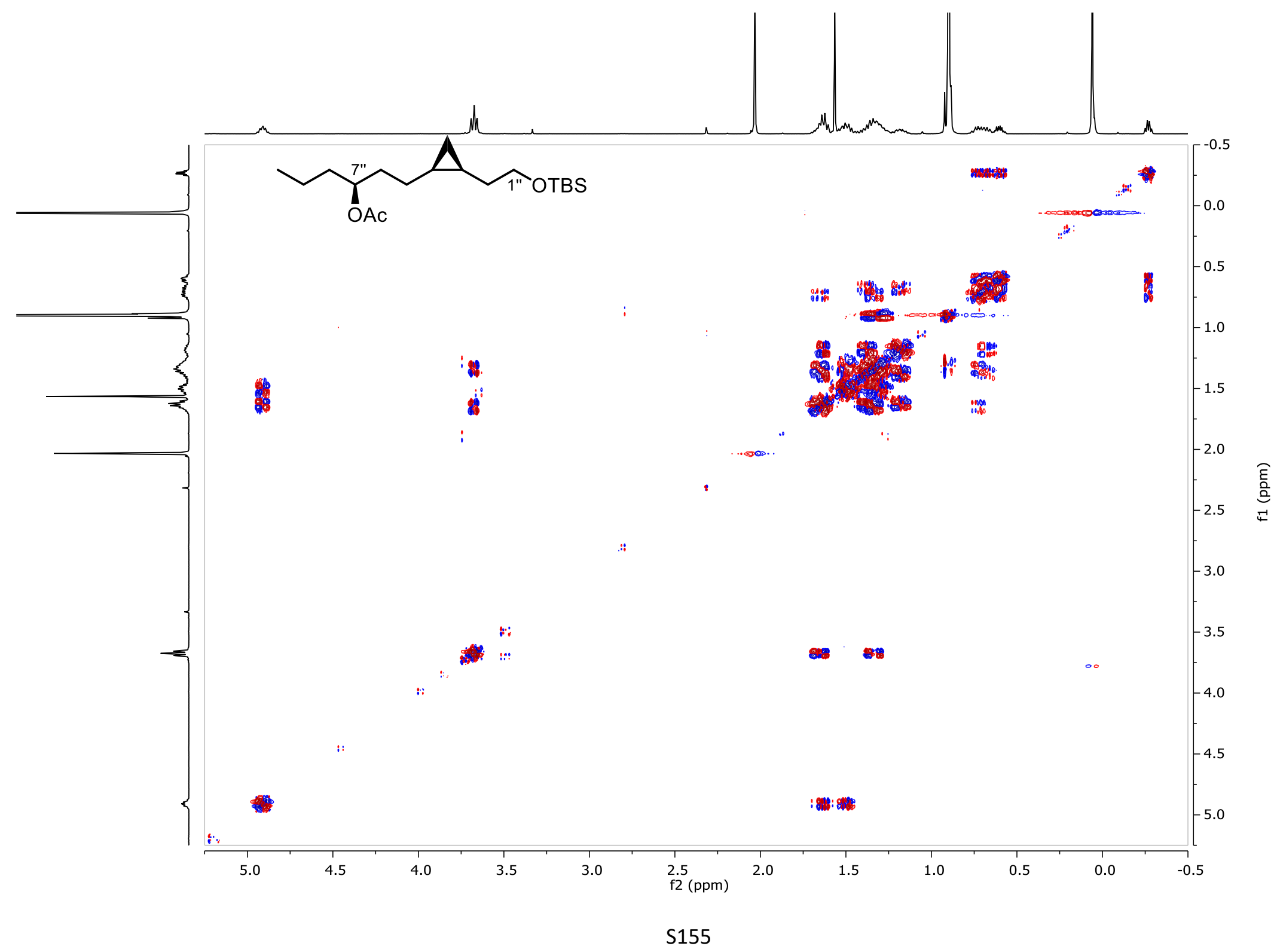


Figure S82: HSQC spectrum (400 MHz, $\left.\mathrm{CDCl}_{3}\right)$ of $(3 S, 4 S, 7 S)-1-O-t e r t-b u t y l d i m e t h y l s i l y l o x y-c i s-3,4-m e t h y l e n e d e c a n-7-y l$ acetate (17).

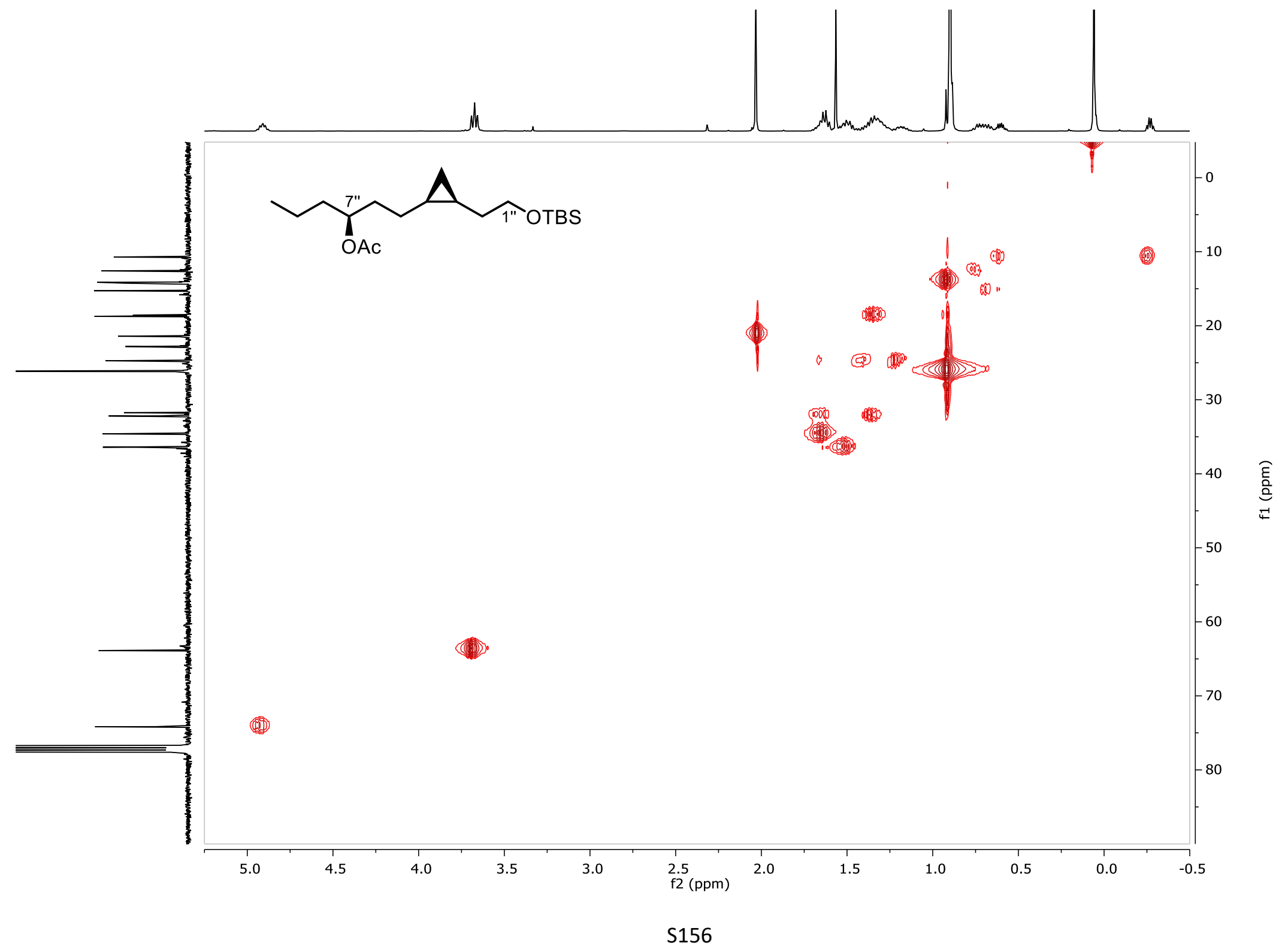


Figure S83: ${ }^{1} \mathrm{H}$ NMR (400 MHz, $\left.\mathrm{CDCl}_{3}\right)$ of (3S,4S,7S)-1-O-tert-butyldimethylsilyloxy-cis-3,4-methylenedecan-7-ol (18).

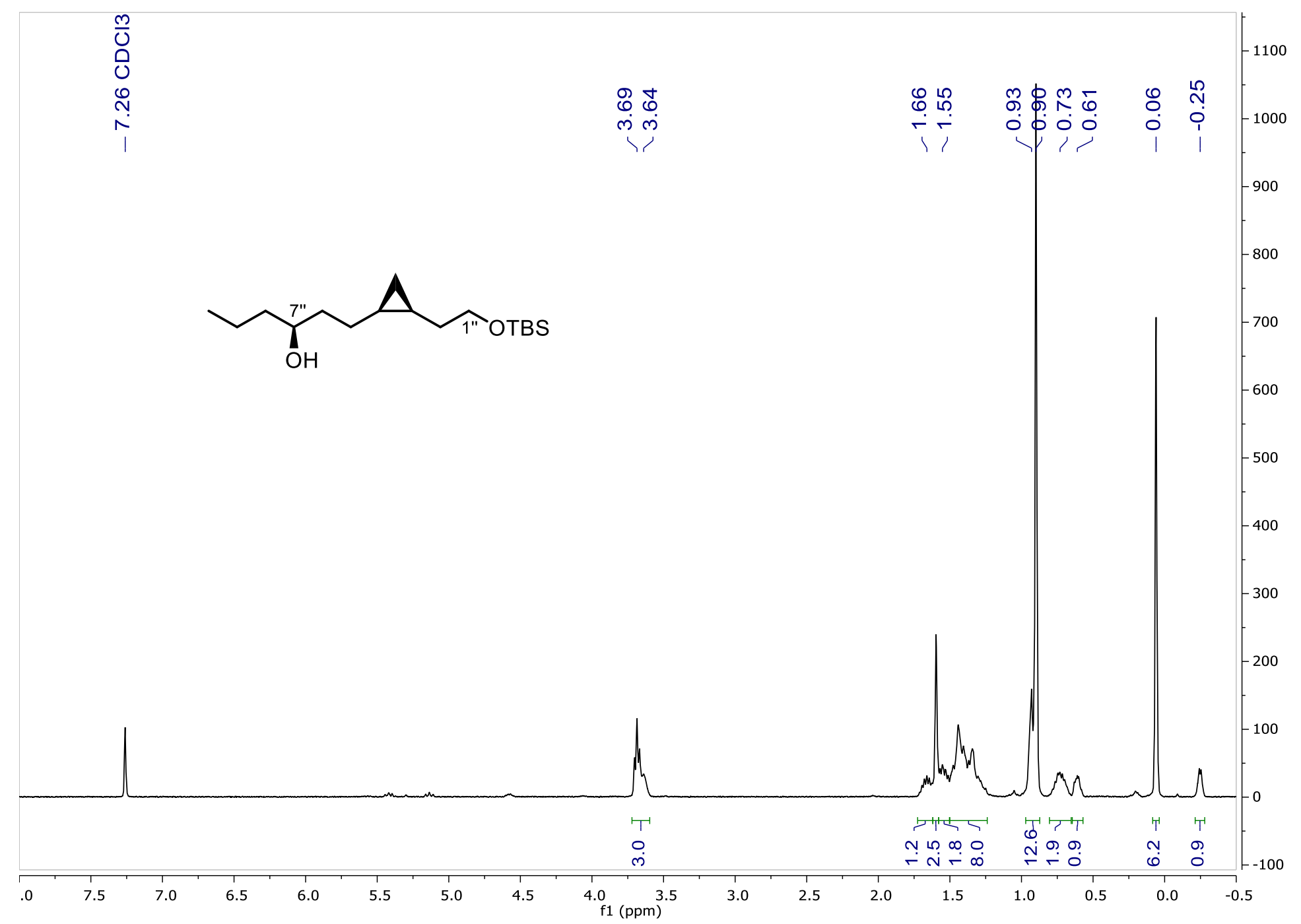


Figure S84: ${ }^{1} \mathrm{H}$ NMR (400 MHz, $\mathrm{CDCl}_{3}$ ) of (3R)-methyl 3-[(3,6-dideoxy- $\alpha$-L-arabino-hexopyranosyl)oxy]-butanoate.

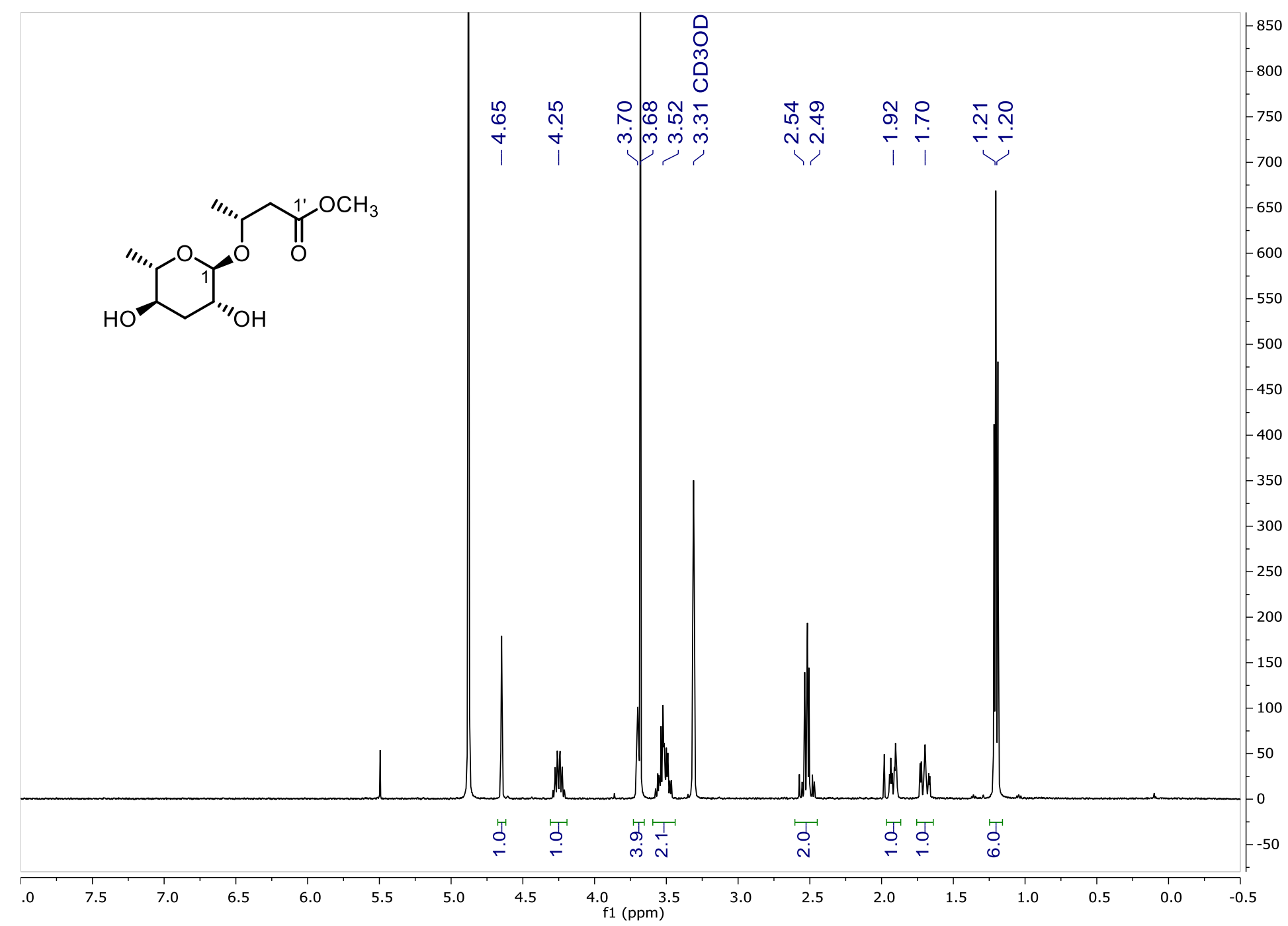


Figure S85: ${ }^{13} \mathrm{C}$ NMR (100 MHz, $\left.\mathrm{CDCl}_{3}\right)$ of (3R)-methyl 3-[(3,6-dideoxy-a-L-arabino-hexopyranosyl)oxy]-butanoate.

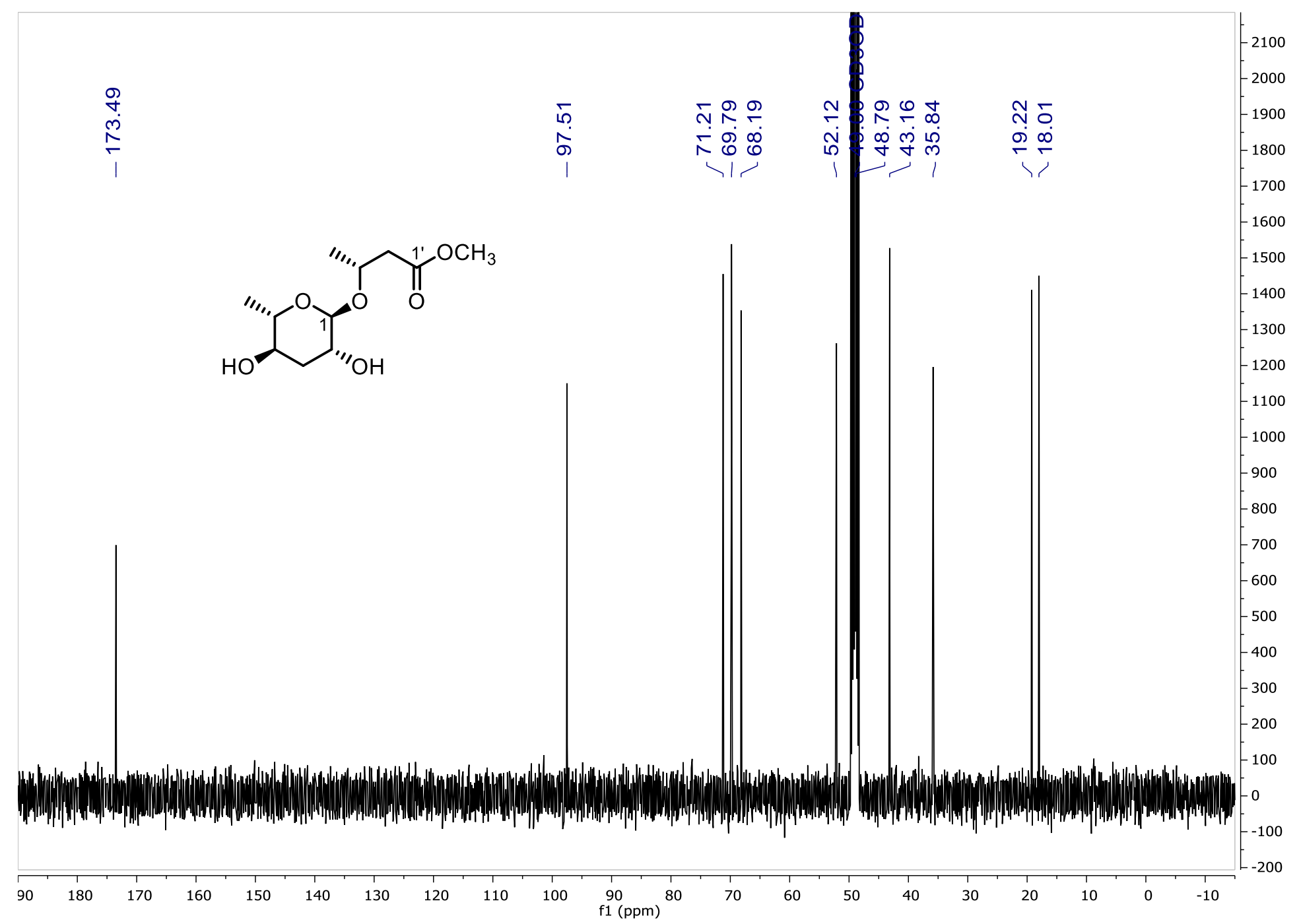


Figure S86: ${ }^{1} \mathrm{H}$ NMR (400 MHz, $\left.\mathrm{CD}_{3} \mathrm{OD}\right)$ of (3R)-methyl 3-[(2,4-di-O-tert-butyldimethylsilyloxy-3,6-dideoxy-a-L-arabinohexopyranosyl)oxy]-butanoate.

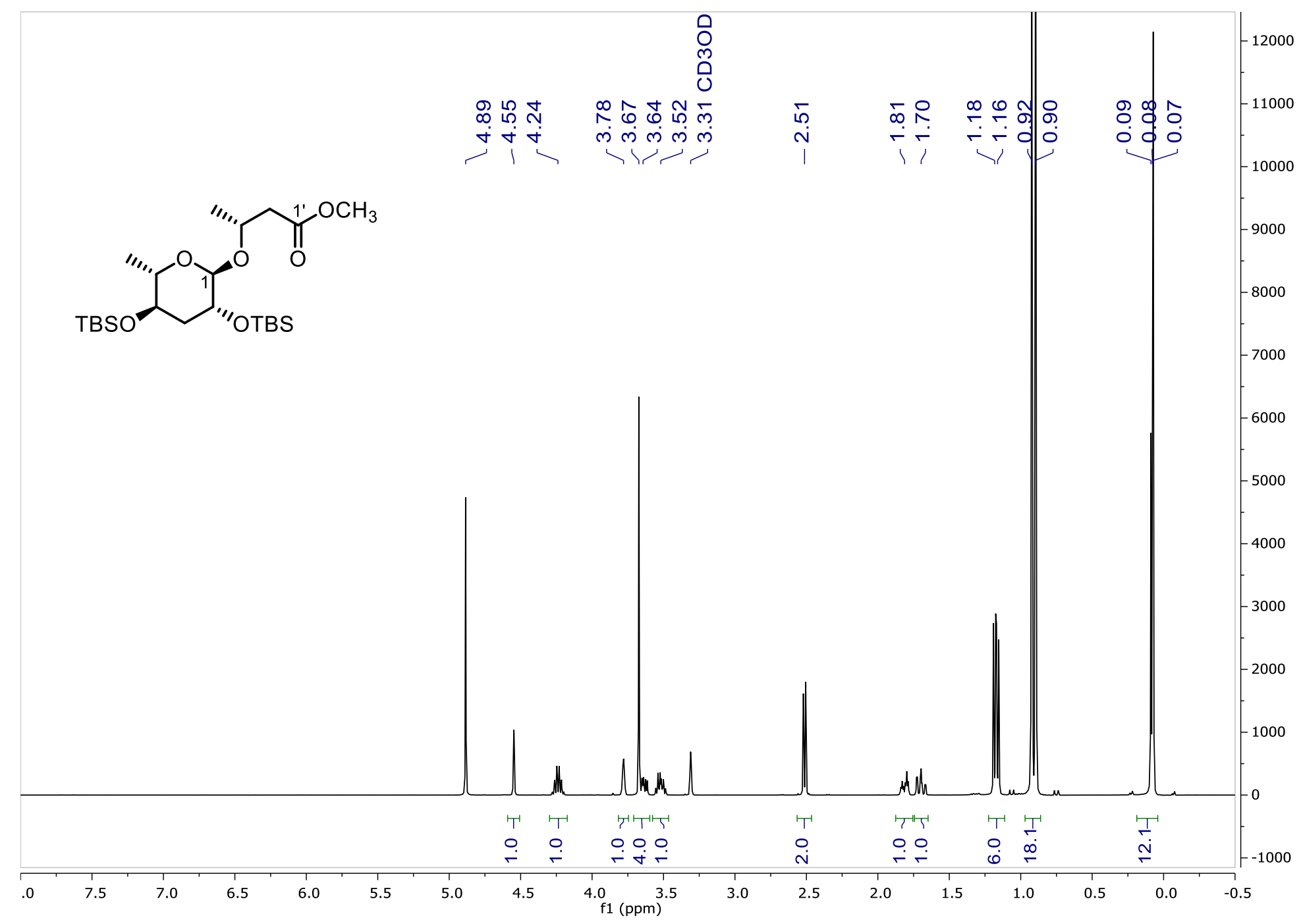


Figure S87: ${ }^{13} \mathrm{C}$ NMR (100 MHz, CD $\left.{ }_{3} \mathrm{OD}\right)$ of (3R)-methyl 3-[(2,4-di-O-tert-butyldimethylsilyloxy-3,6-dideoxy-a-L-arabinohexopyranosyl)oxy]-butanoate.

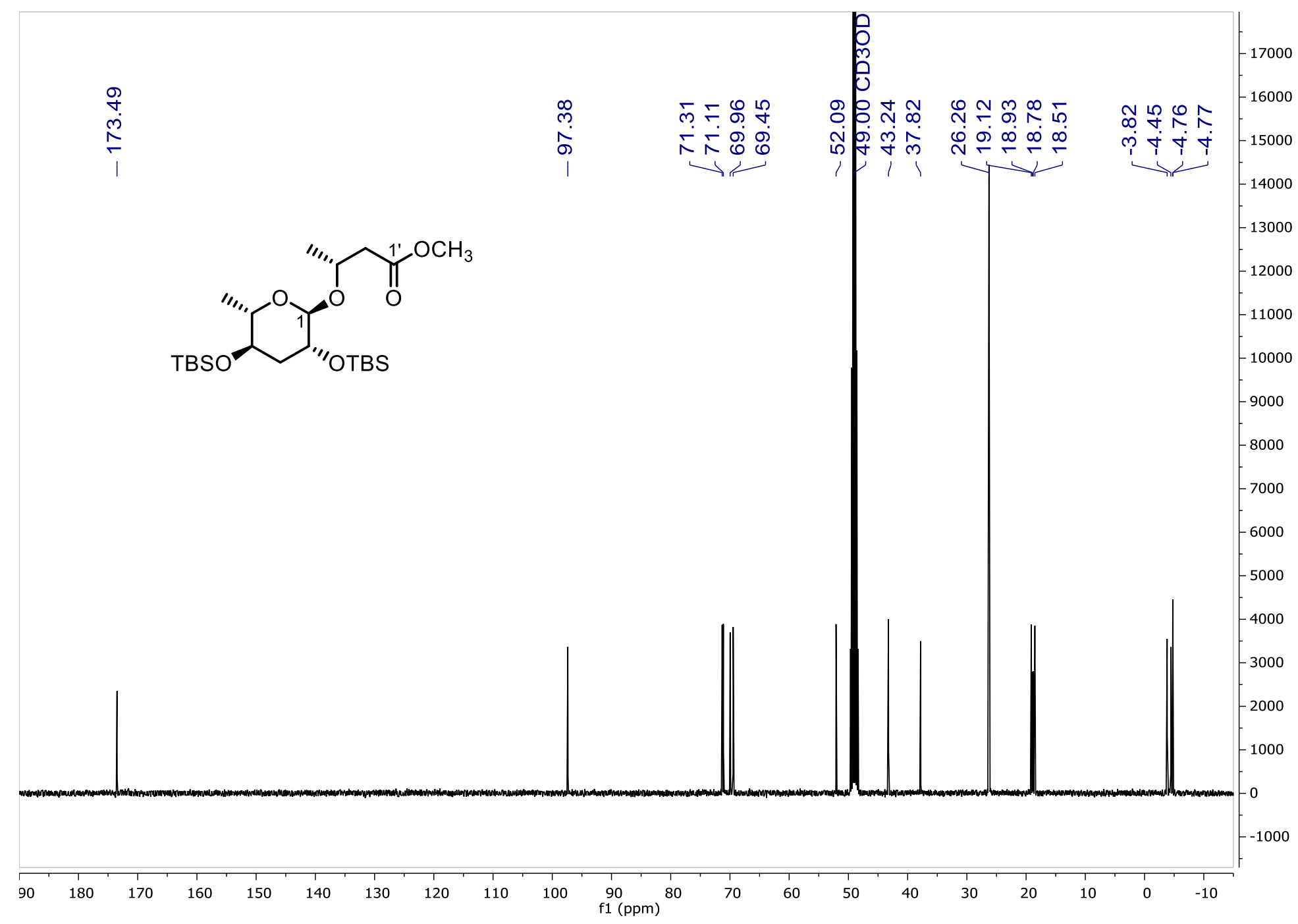


Figure S88: dqf-COSY (400 MHz, $\left.\mathrm{CD}_{3} \mathrm{OD}\right)$ of (3R)-methyl 3-[(2,4-di-O-tert-butyldimethylsilyloxy-3,6-dideoxy- $\alpha$-L-arabinohexopyranosyl)oxy]-butanoate.

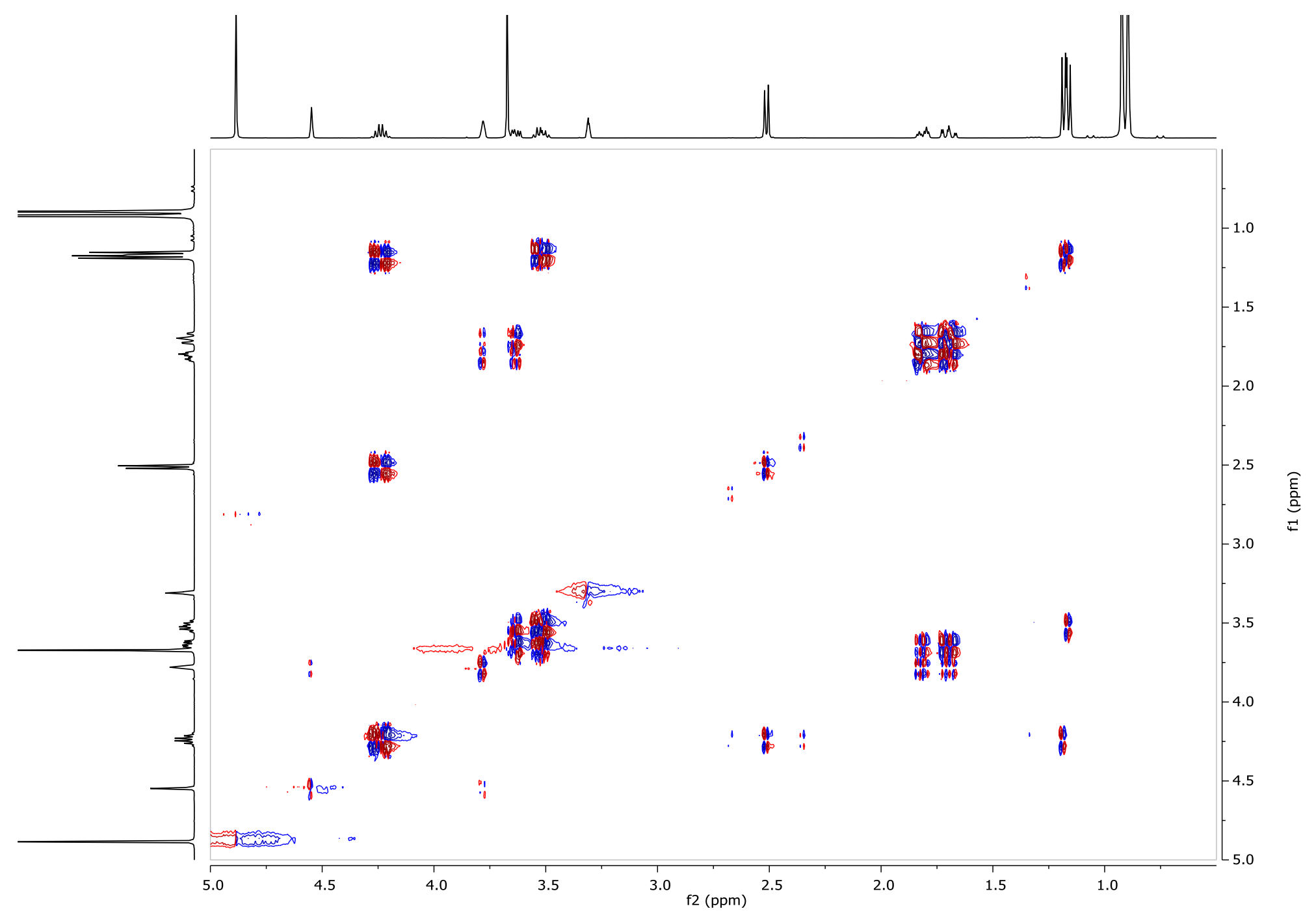


Figure S89: ${ }^{1} \mathrm{H}$ NMR $\left(400 \mathrm{MHz}, \mathrm{CDCl}_{3}\right)$ of (3R)-[(2,4-di-O-tert-butyldimethylsilyloxy-3,6-dideoxy-a-L-arabino-hexopyranosyl)oxy]butanoic acid (20).

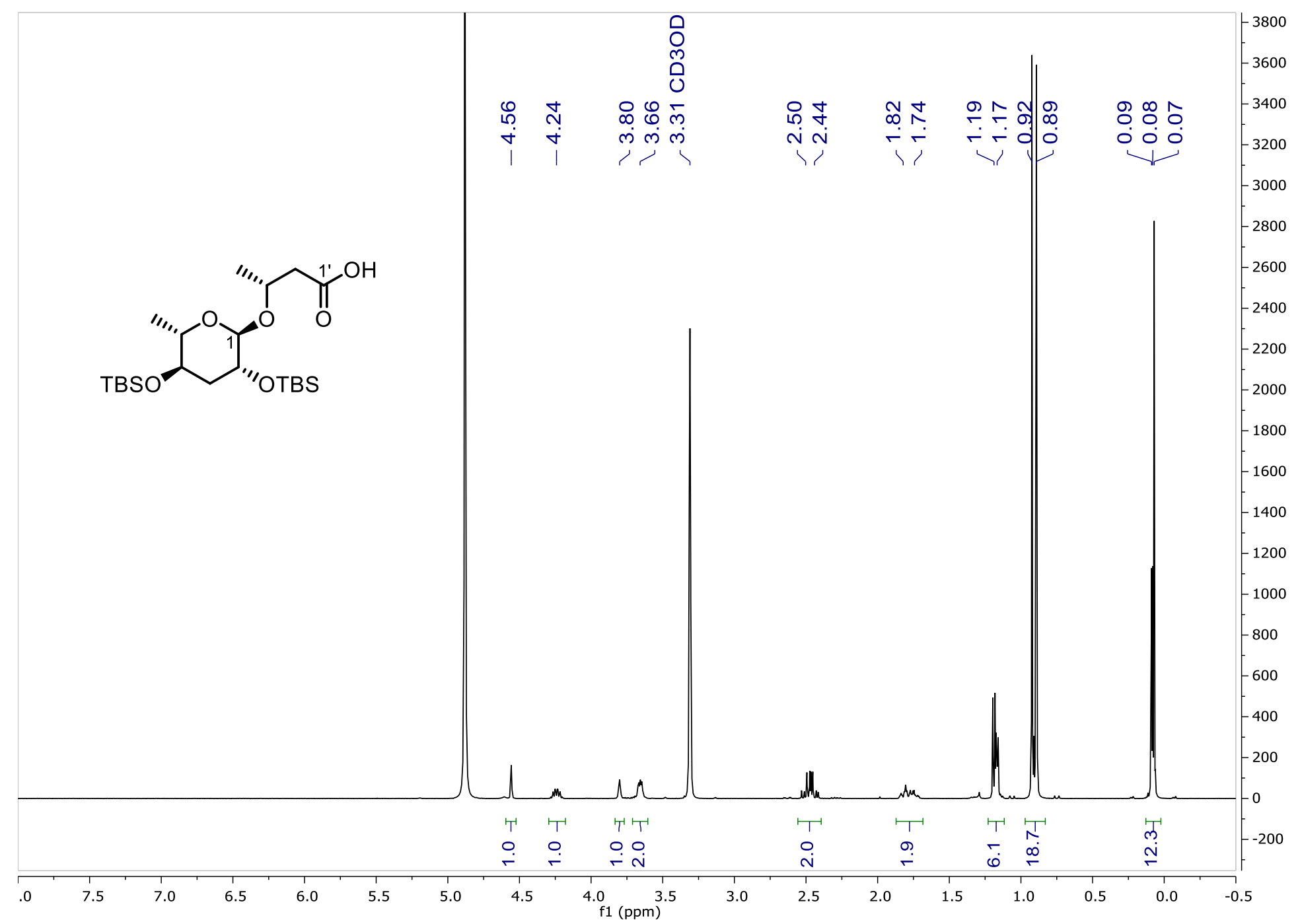


Figure S90: ${ }^{1} \mathrm{H}$ NMR (400 MHz, $\left.\mathrm{CDCl}_{3}\right)$ of (3'R,3S,4S,7S)-7-[(3'-[(2,4-di-O-tert-butyldimethylsilyloxy-3",6"'-dideoxy-a-L-arabinohexopyranosyl)-oxy]-butanoyl)oxy]-3,4-cis-methylene-1-O-tert-butyldimethylsilyloxy decane (21)

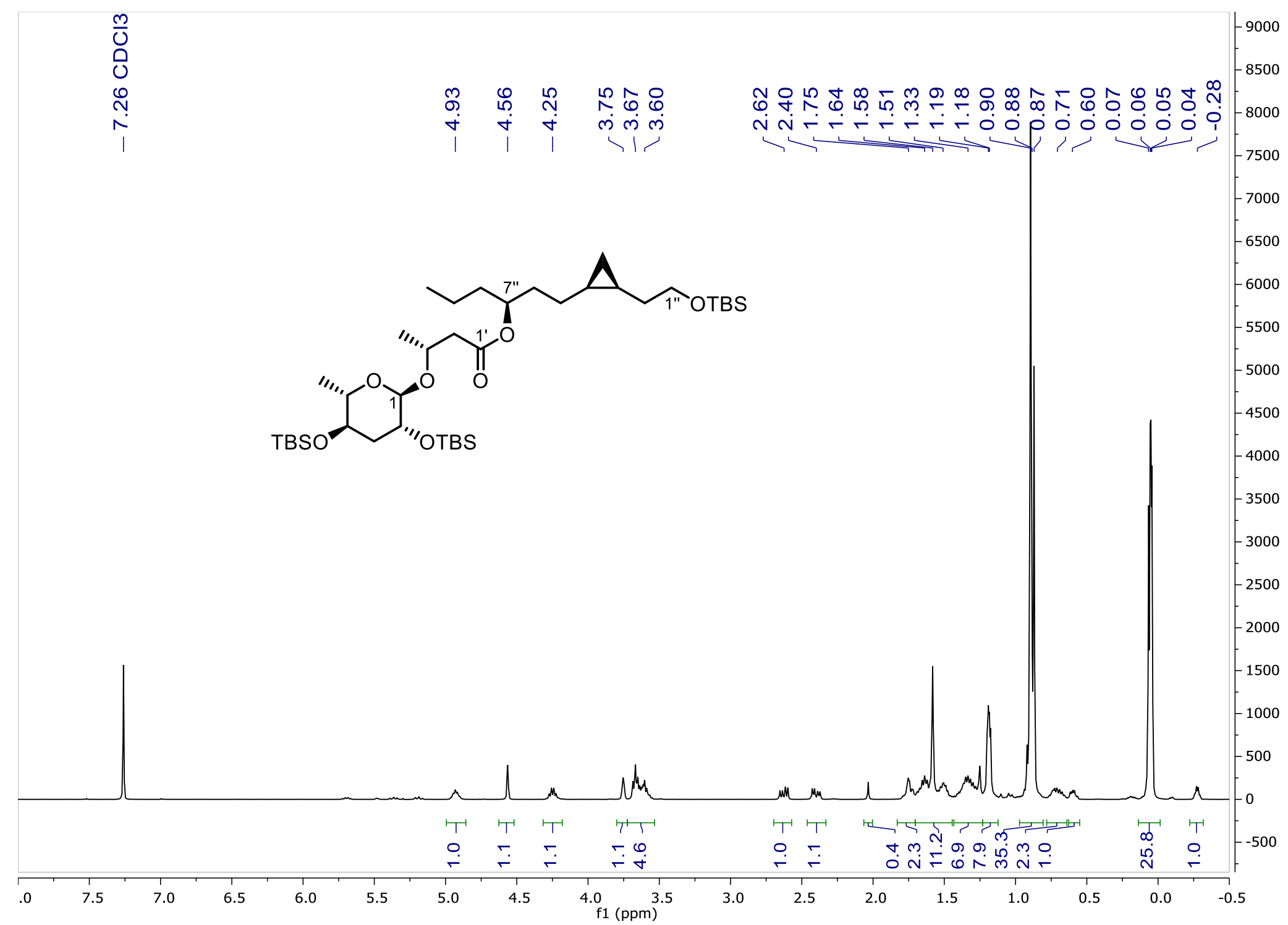


Figure S91: ${ }^{1} \mathrm{H}$ NMR $\left(400 \mathrm{MHz}, \mathrm{CD}_{3} \mathrm{OD}\right)$ of (3'R,3S,4S,7S)-7-[(3'-[(2,4-di-O-tert-butyldimethylsilyloxy-3",6"-dideoxy-a-L-arabinohexopyranosyl)-oxy]-butanoyl)oxy]-3,4-cis-methylene-1-O-tert-butyldimethylsilyloxy decane (21)

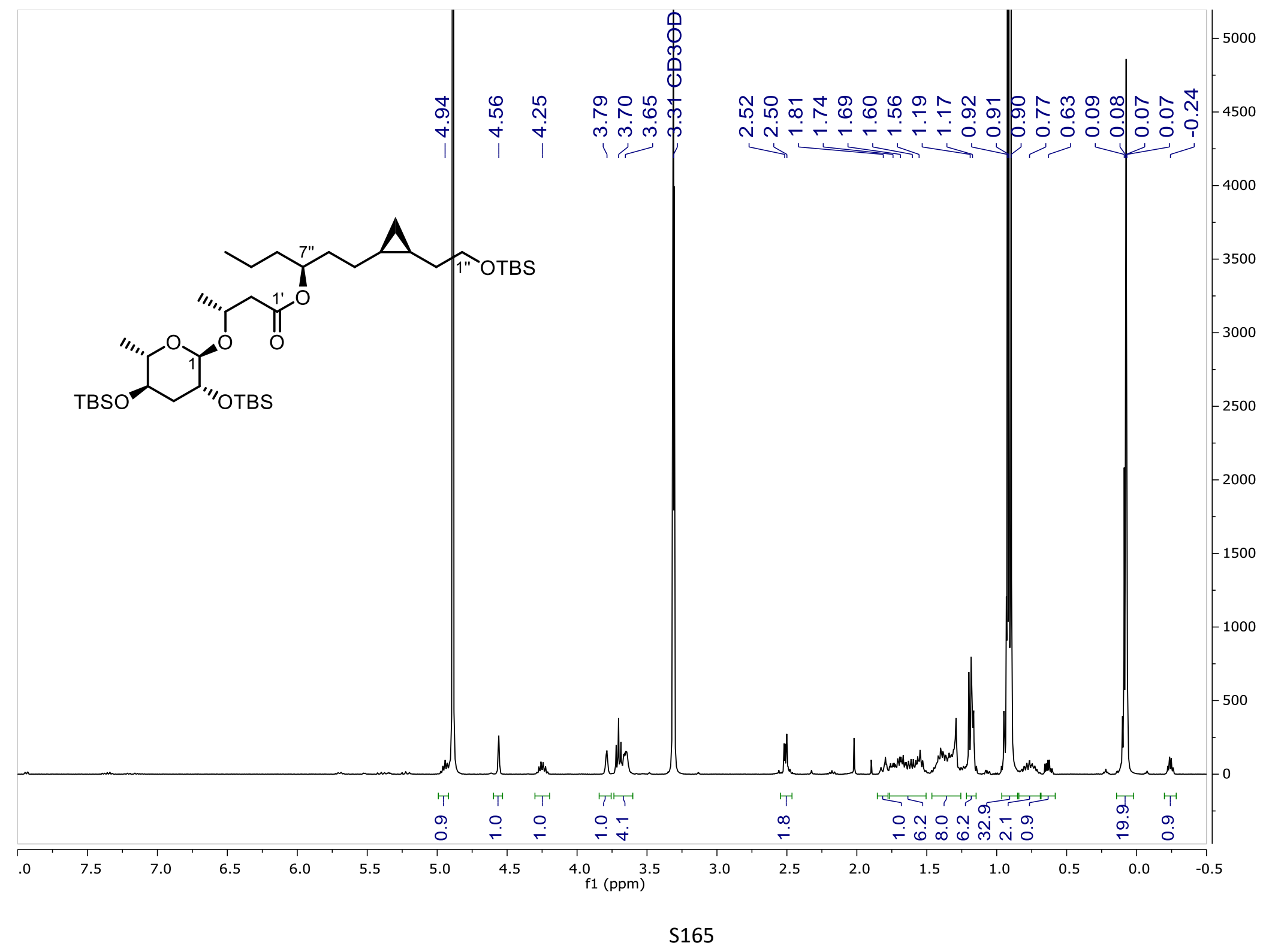


Figure S92: ${ }^{13} \mathrm{C}$ NMR $\left(100 \mathrm{MHz}, \mathrm{CDCl}_{3}\right)$ of (3'R,3S,4S,7S)-7-[(3'-[(2,4-di-O-tert-butyldimethylsilyloxy-3",6"'-dideoxy-a-L-arabinohexopyranosyl)-oxy]-butanoyl)oxy]-3,4-cis-methylene-1-O-tert-butyldimethylsilyloxy decane (21)

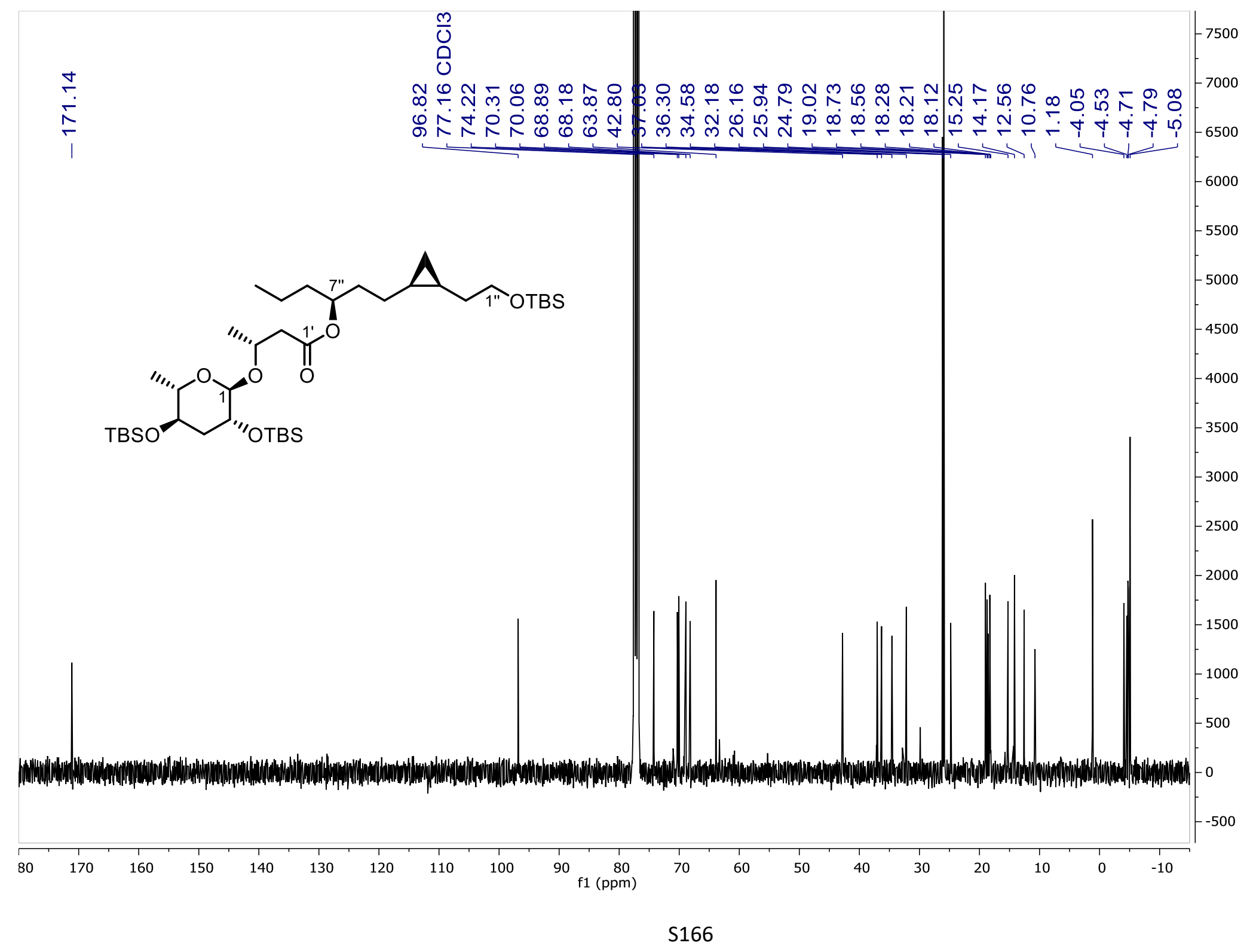


Figure S93: dqf-COSY (400 MHz, CDCl 3 ) of (3'R,3S,4S,7S)-7-[(3'-[(2,4-di-O-tert-butyldimethylsilyloxy-3",6"'-dideoxy-a-L-arabinohexopyranosyl)-oxy]-butanoyl)oxy]-3,4-cis-methylene-1-O-tert-butyldimethylsilyloxy decane (21).

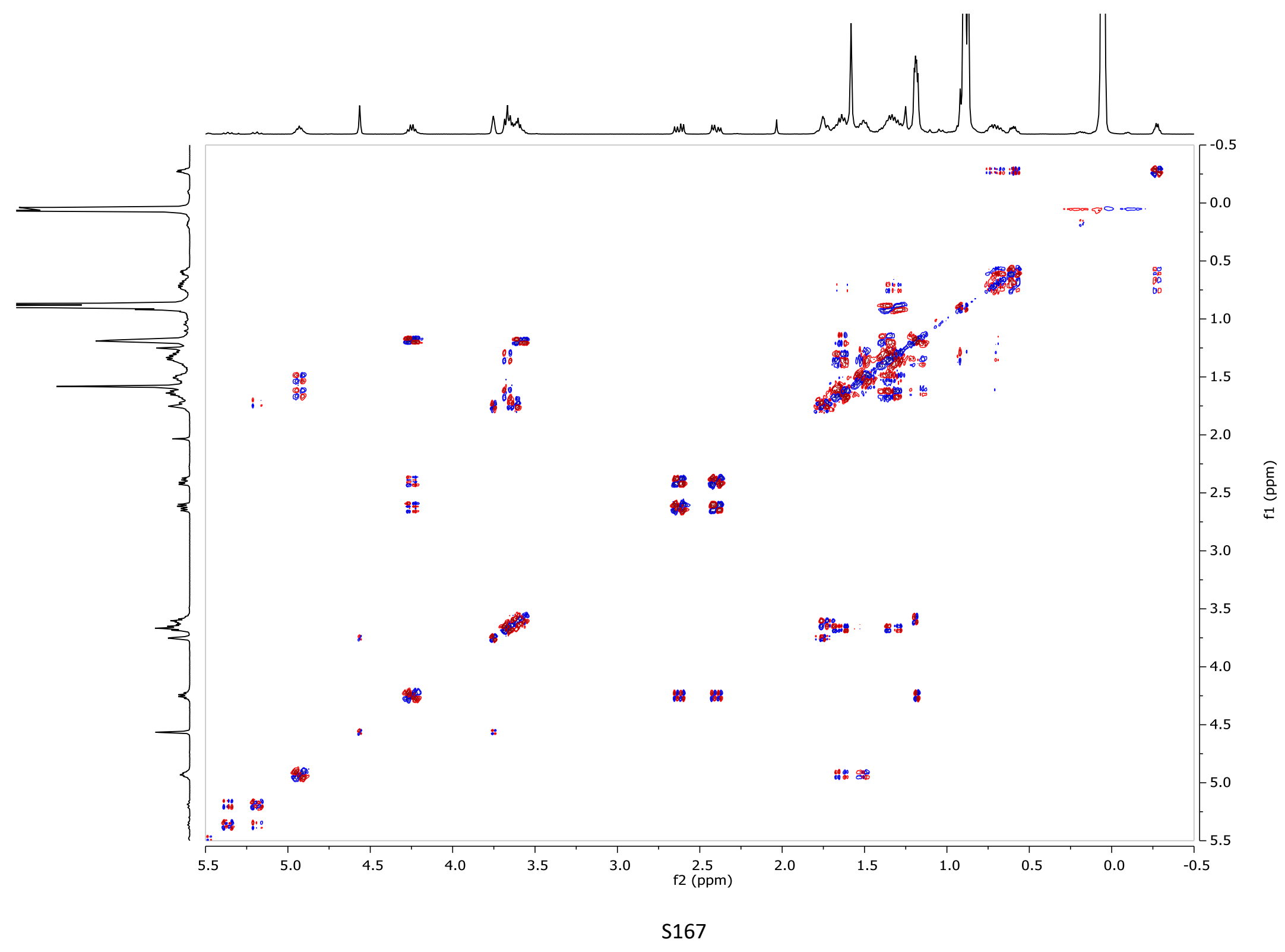


Figure S94: HSQC (400 MHz, $\left.\mathrm{CDCl}_{3}\right)$ of (3'R,3S,4S,7S)-7-[(3'-[(2,4-di-O-tert-butyldimethylsilyloxy-3",6"'-dideoxy-a-L-arabinohexopyranosyl)-oxy]-butanoyl)oxy]-3,4-cis-methylene-1-O-tert-butyldimethylsilyloxy decane (21).

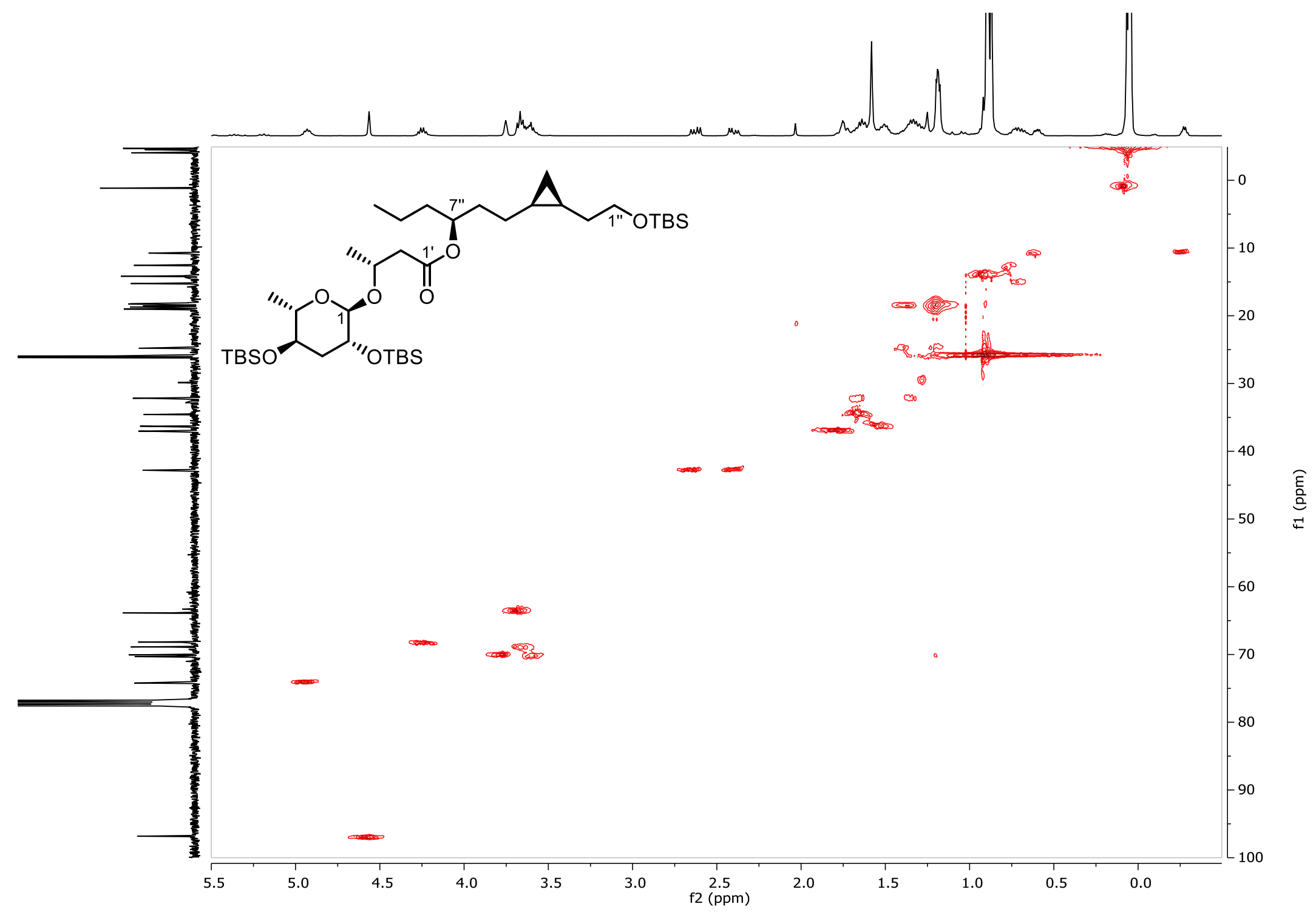


Figure S95: ${ }^{1} \mathrm{H}$ NMR (400 MHz, CD $\left.{ }_{3} \mathrm{OD}\right)$ of (3'R,3S,4S,7S)-7-[(3'-[(3",6"'-dideoxy-a-L-arabino-hexopyranosyl)-oxy]-butanoyl)oxy]-3,4cis-methylene-1-decanol (22)

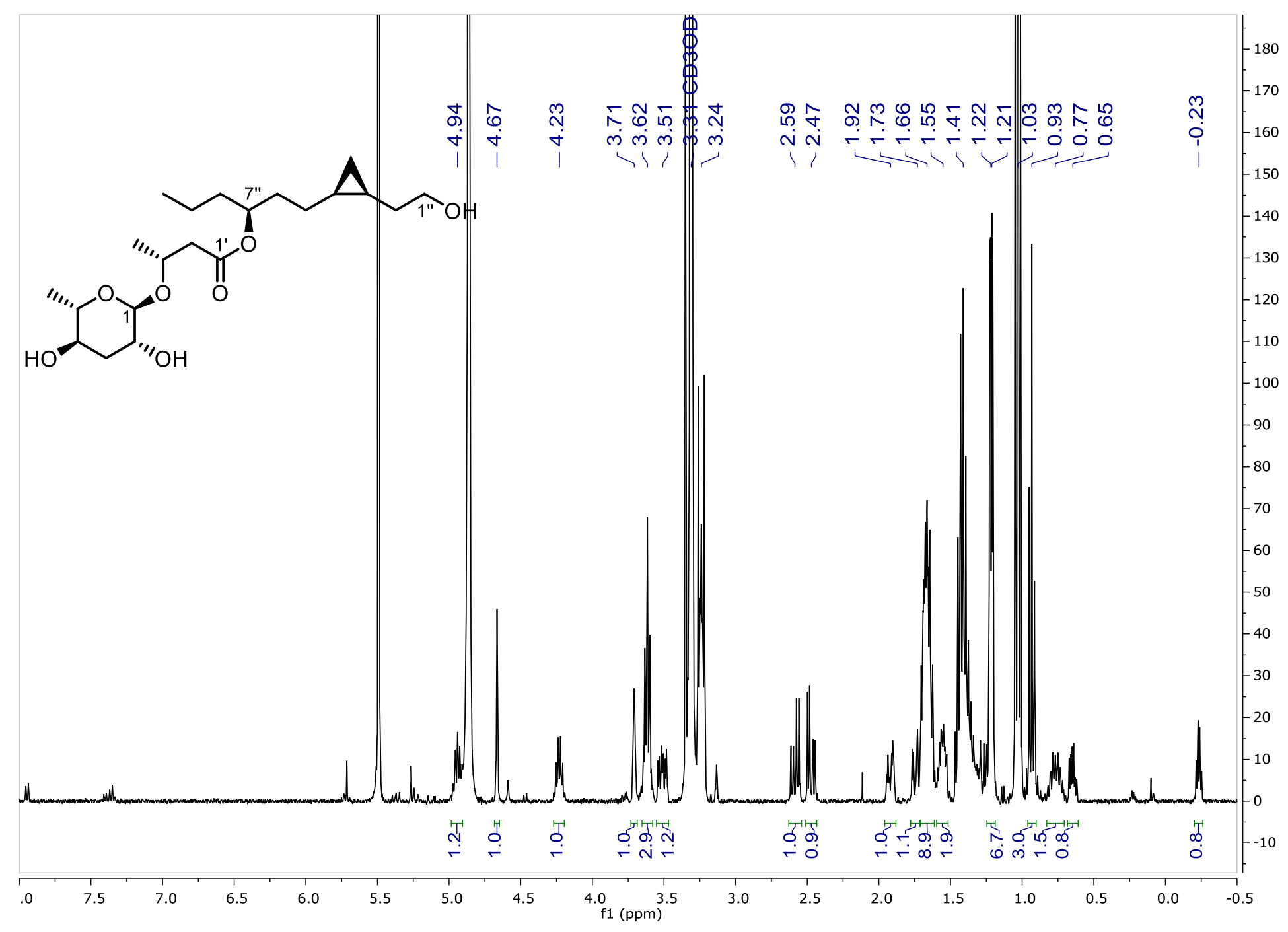


Figure S96: dqf-COSY (400 MHz, CD 3 OD) of (3'R,3S,4S,7S)-7-[(3'-[(3",6"-dideoxy-a-L-arabino-hexopyranosyl)-oxy]-butanoyl)oxy]3,4-cis-methylene-1-decanol (22)

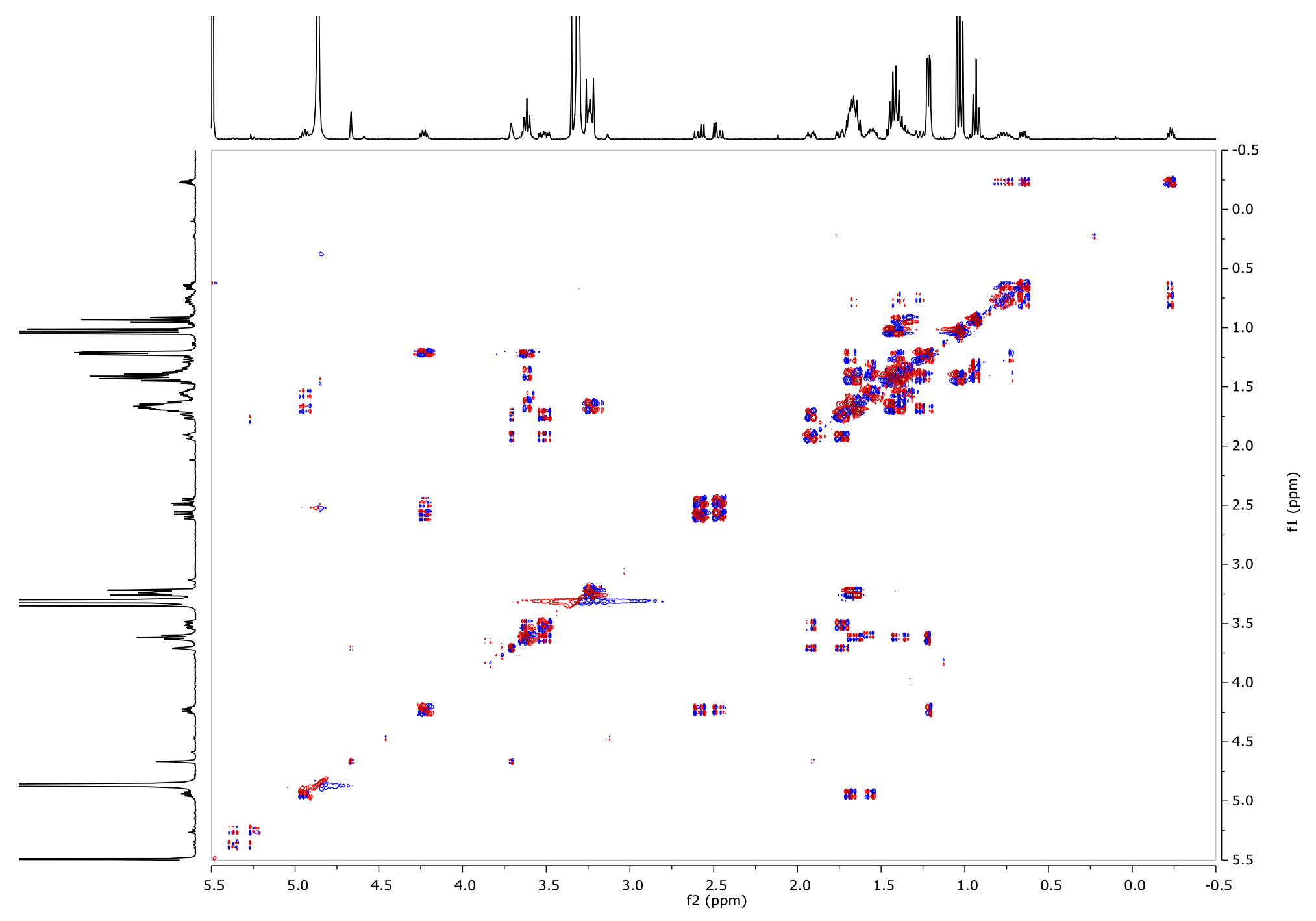


Figure S97: HSQC (400 MHz, CD $\left.{ }_{3} \mathrm{OD}\right)$ of (3'R,3S,4S,7S)-7-[(3'-[(3',6"'-dideoxy-a-L-arabino-hexopyranosyl)-oxy]-butanoyl)oxy]-3,4cis-methylene-1-decanol (22)

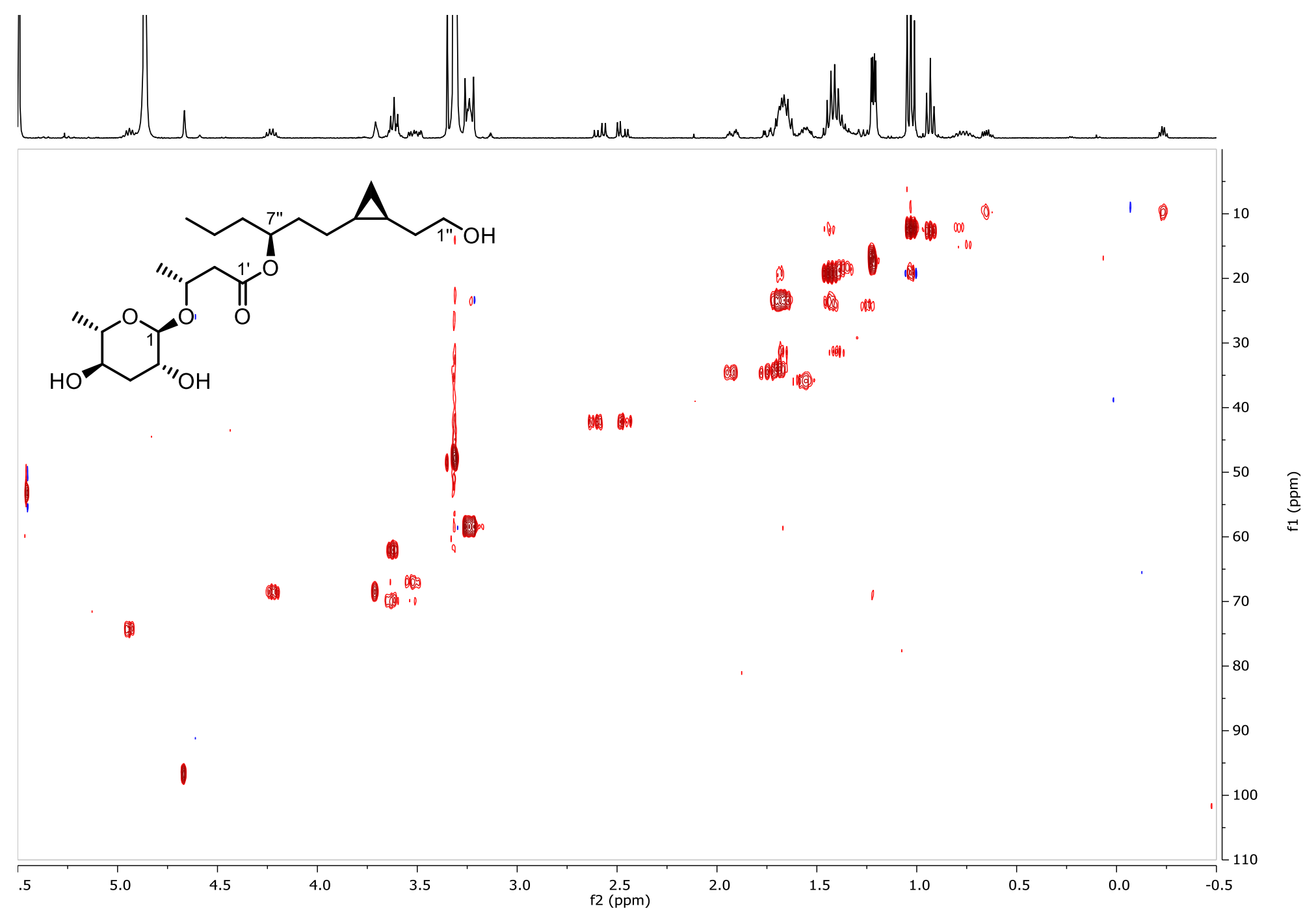


Figure S98: 'H NMR (700 MHz, CD 3 OD) of synthetic (3'R,3S,4S,7S)-7-[(3'-[(3"',6"-dideoxy-a-L-arabino-hexopyranosyl)-oxy]butanoyl)oxy]-3,4-cis-methylene decanoic acid (fasc\#1, 3)

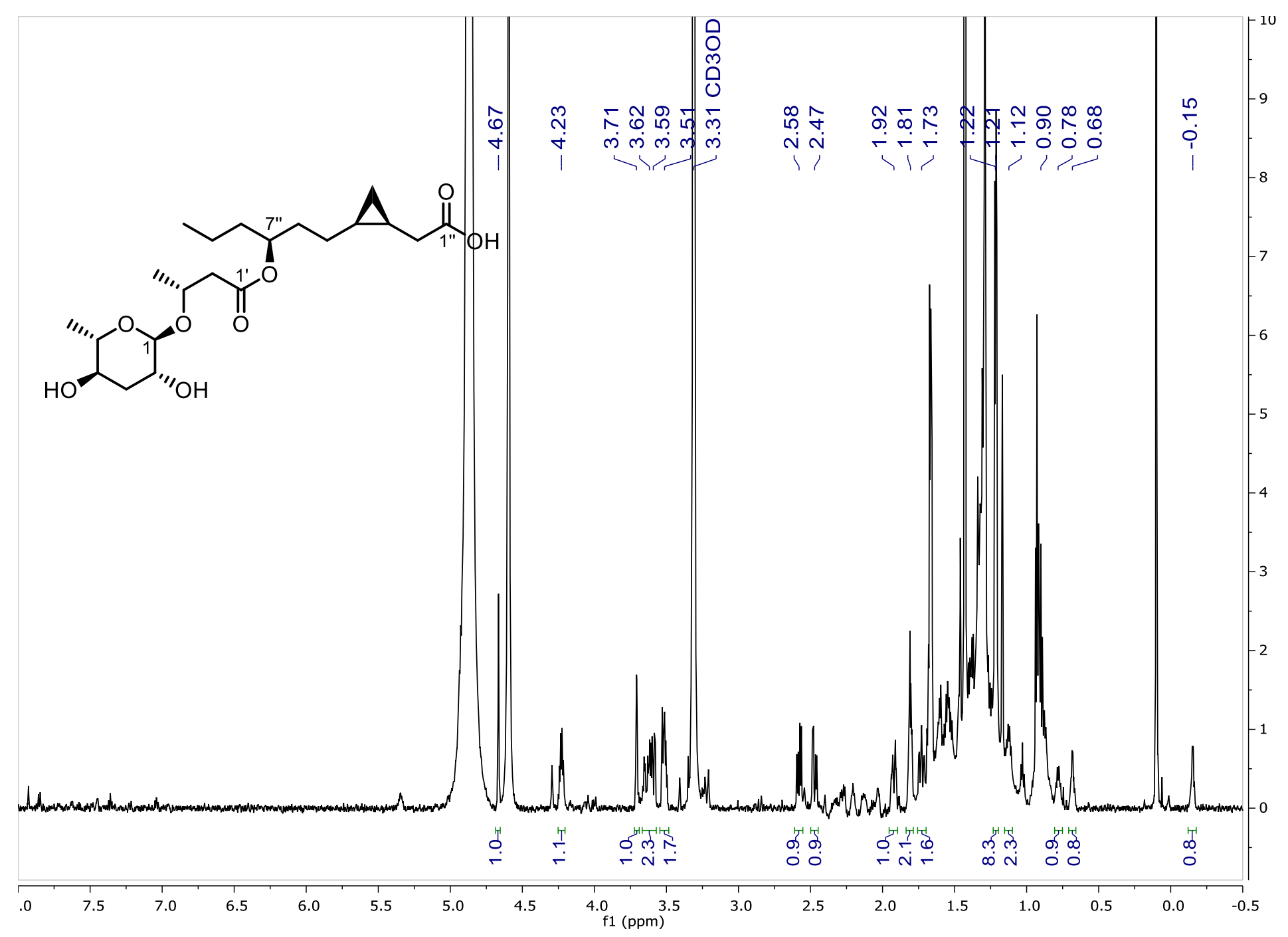


Figure S99: dqf-COSY (700 MHz, CD $\left.{ }_{3} \mathrm{OD}\right)$ of synthetic (3'R,3S,4S,7S)-7-[(3'-[(3",6"'-dideoxy-a-L-arabino-hexopyranosyl)-oxy]butanoyl)oxy]-3,4-cis-methylene decanoic acid (fasc\#1, 3)

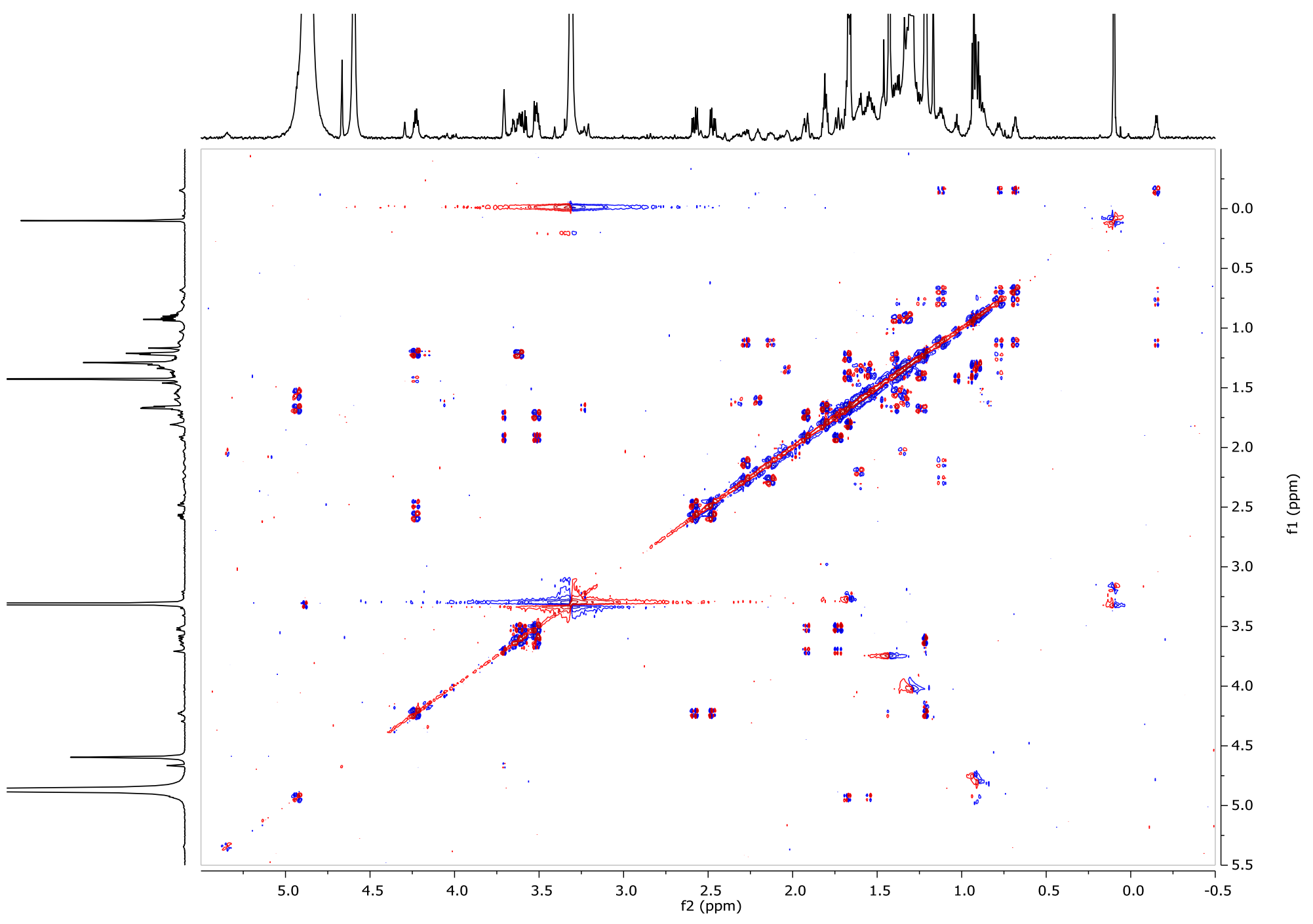


Figure S100 : HSQC spectrum (700 MHz, CD $\left.{ }_{3} \mathrm{OD}\right)$ of synthetic (3'R,3S,4S,7S)-7-[(3'-[(3",6"'-dideoxy-a-L-arabino-hexopyranosyl)oxy]-butanoyl)oxy]-3,4-cis-methylene decanoic acid (fasc\#1, 3)

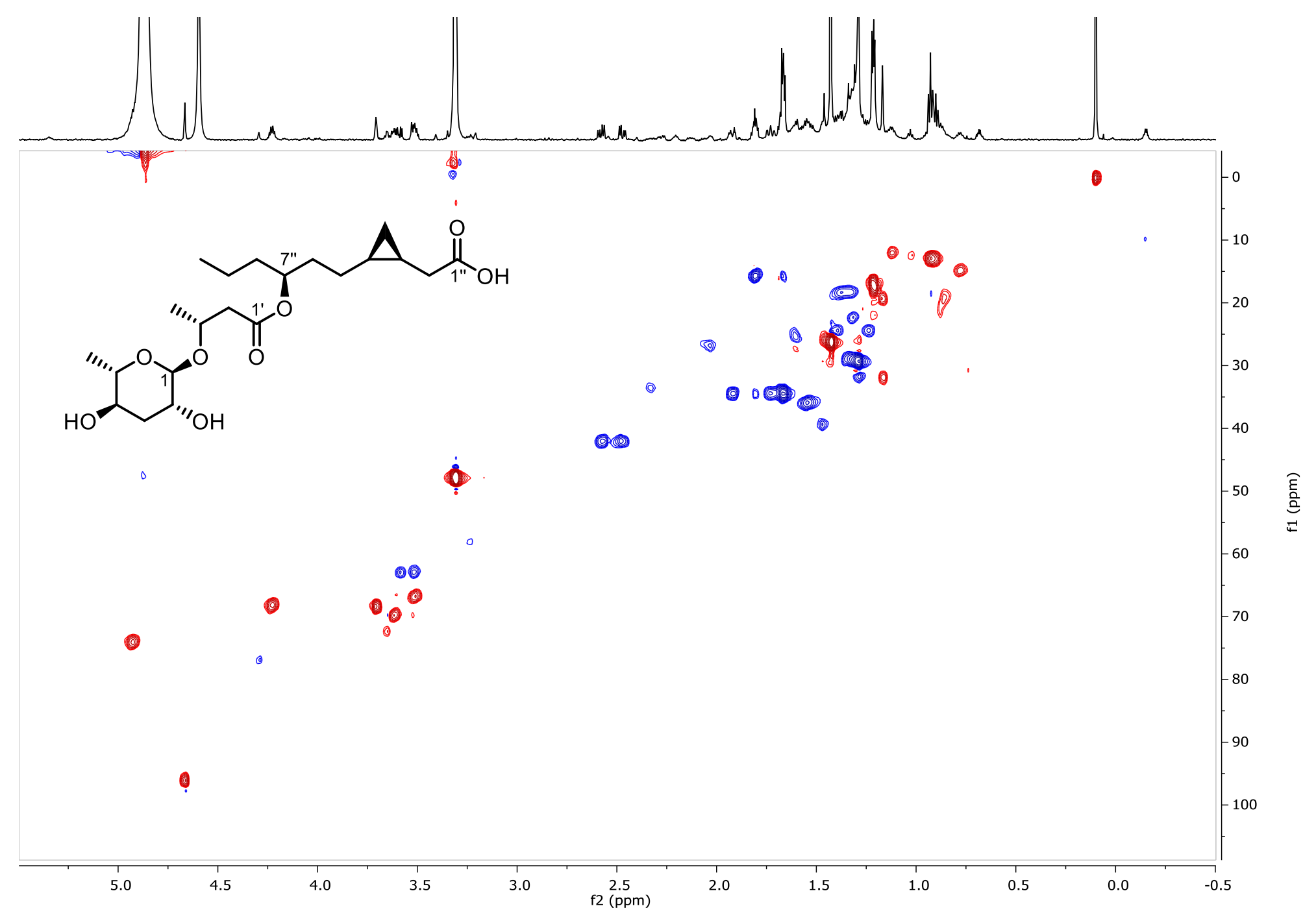


Figure S101: $\mathrm{HMBC}\left(700 \mathrm{MHz}, \mathrm{CD}_{3} \mathrm{OD}\right)$ of synthetic (3'R,3S,4S,7S)-7-[(3'-[(3",6"'-dideoxy-a-L-arabino-hexopyranosyl)-oxy]butanoyl)oxy]-3,4-cis-methylene decanoic acid (fasc\#1, 3)

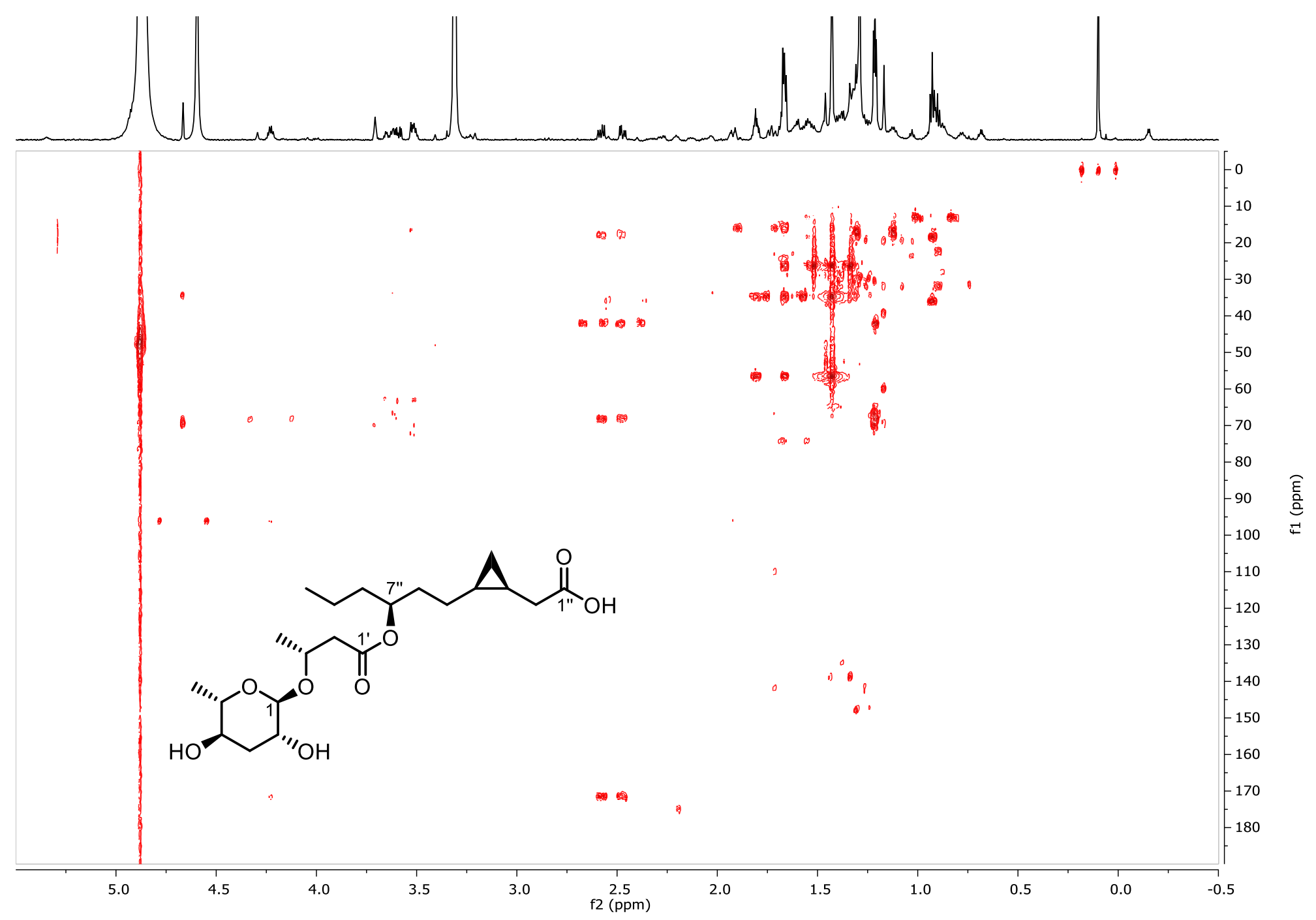


Figure S102: ${ }^{\mathrm{H}} \mathrm{NMR}\left(400 \mathrm{MHz}, \mathrm{CD}_{3} \mathrm{OD}\right)$ of synthetic (3'R,3S,4S,7S)-7-[(3'-[(3",6"'-dideoxy-a-L-arabino-hexopyranosyl)-oxy]butanoyl)oxy]-3,4-cis-methylene decanoic acid (fasc\#1, 3)

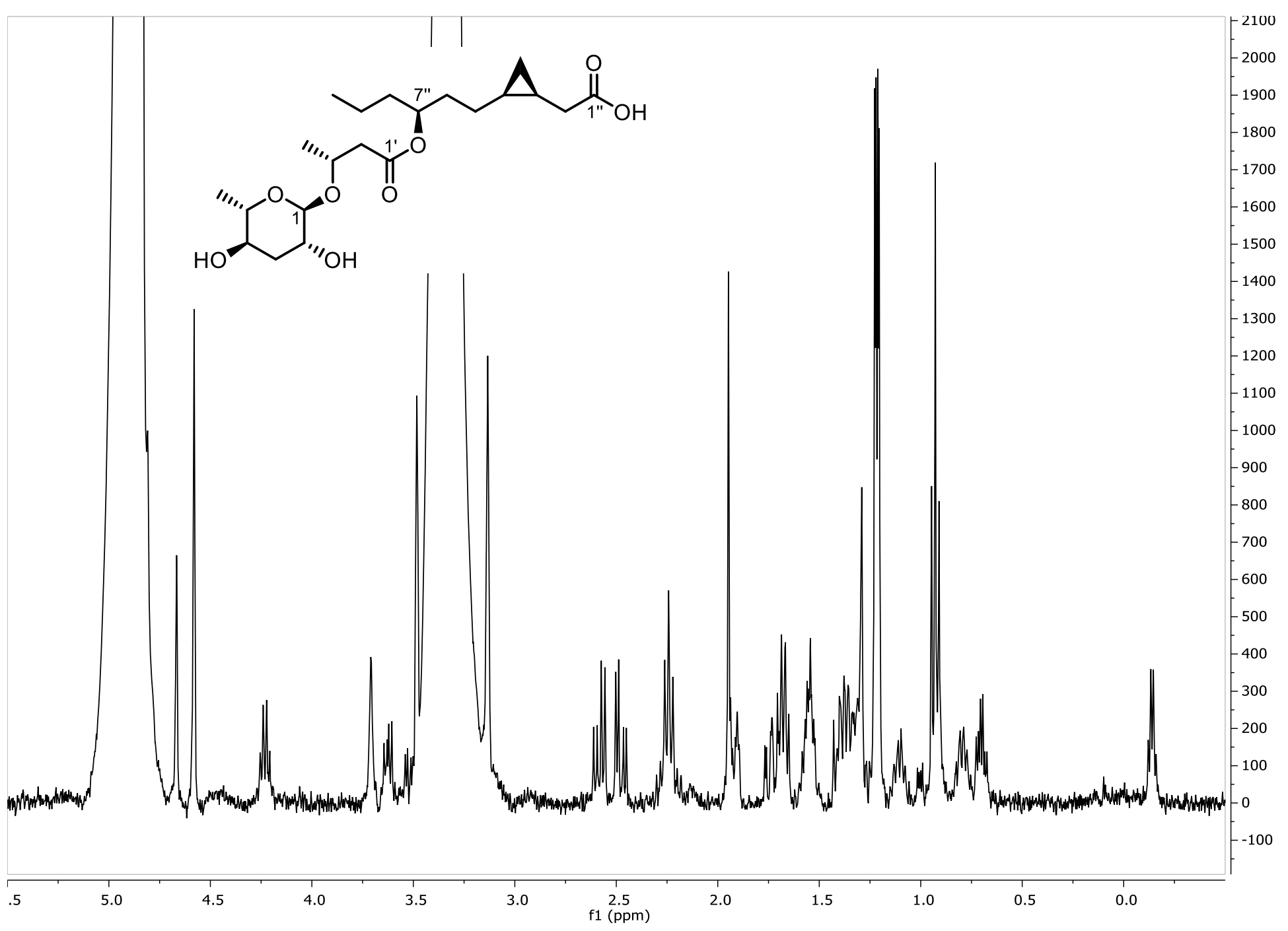


Figure S103: ${ }^{1} \mathrm{H}$ NMR (400 MHz, CD $\left.{ }_{3} \mathrm{OD}\right)$ of natural (3'R,3S,4S,7S)-7-[(3'-[(3", 6"-dideoxy-a-L-arabino-hexopyranosyl)-oxy]butanoyl)oxy]-3,4-cis-methylene decanoic acid (fasc\#1, 3) from C. remanei PB4641

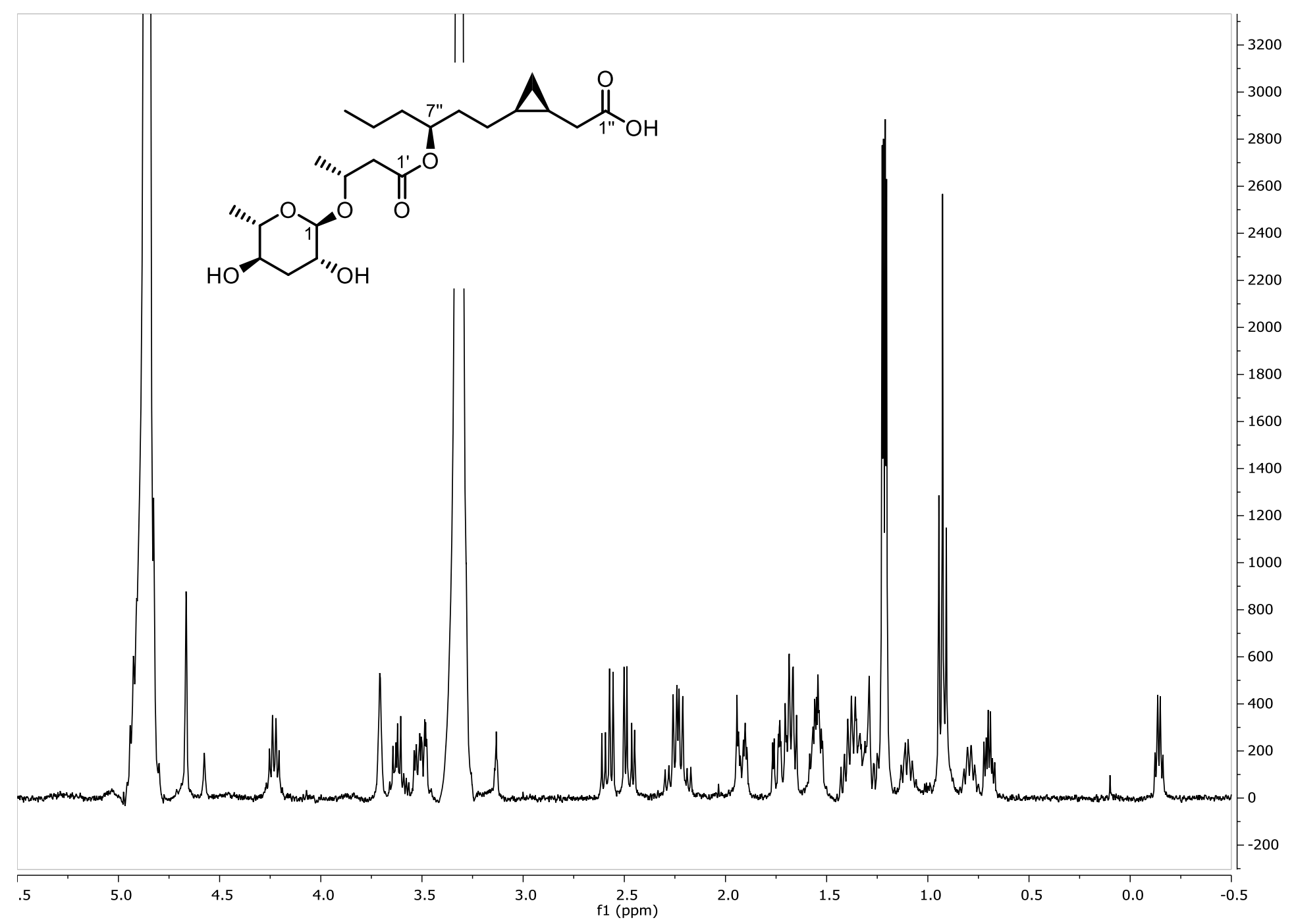


Figure S104: ${ }^{1} \mathrm{H}$ NMR (400 MHz, $\left.\mathrm{CDCl}_{3}\right)$ of methyl cis-9-hexadecenoate.

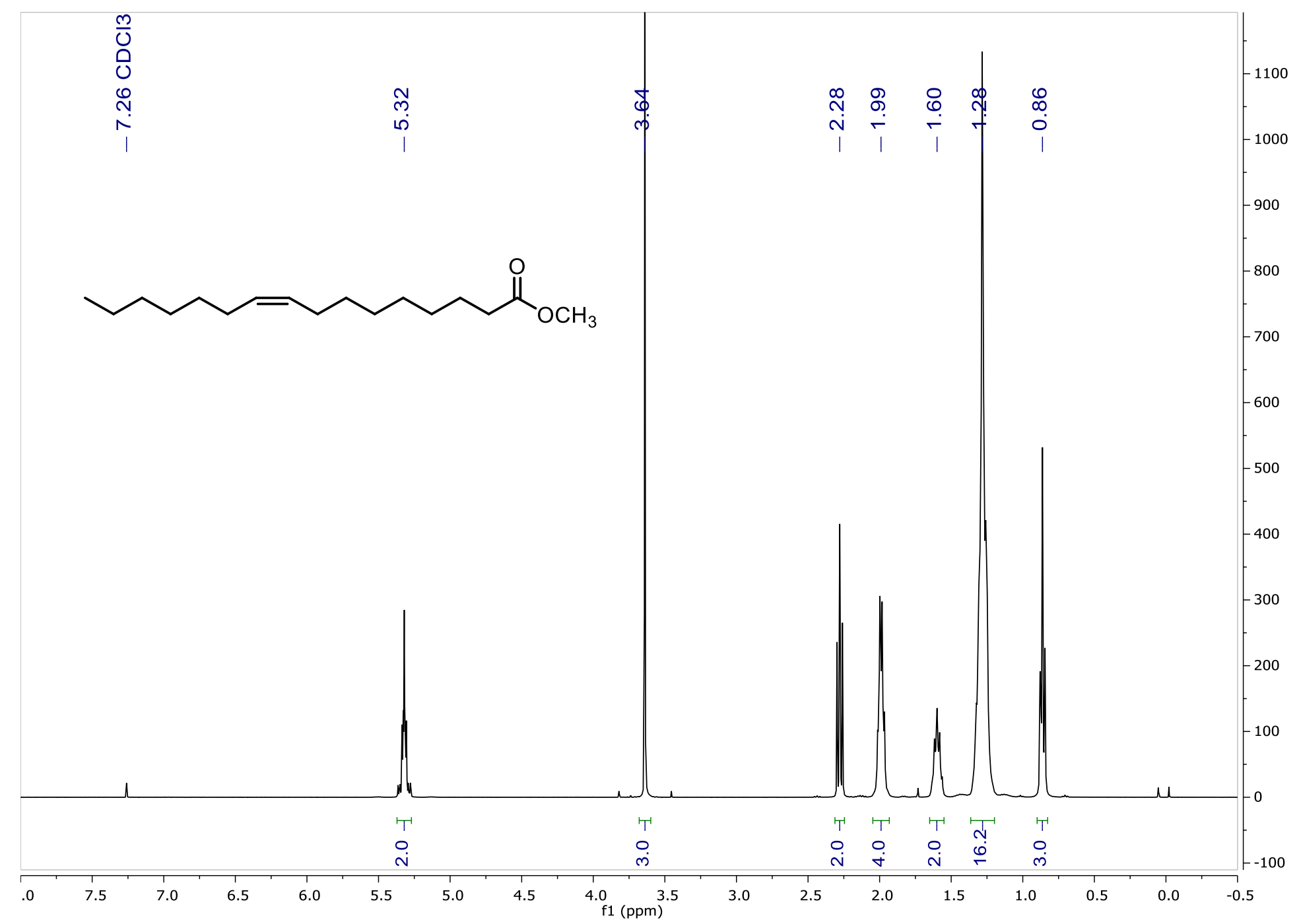


Figure S105: ${ }^{13} \mathrm{C}$ NMR (100 MHz, $\mathrm{CDCl}_{3}$ ) of methyl cis-9-hexadecenoate.

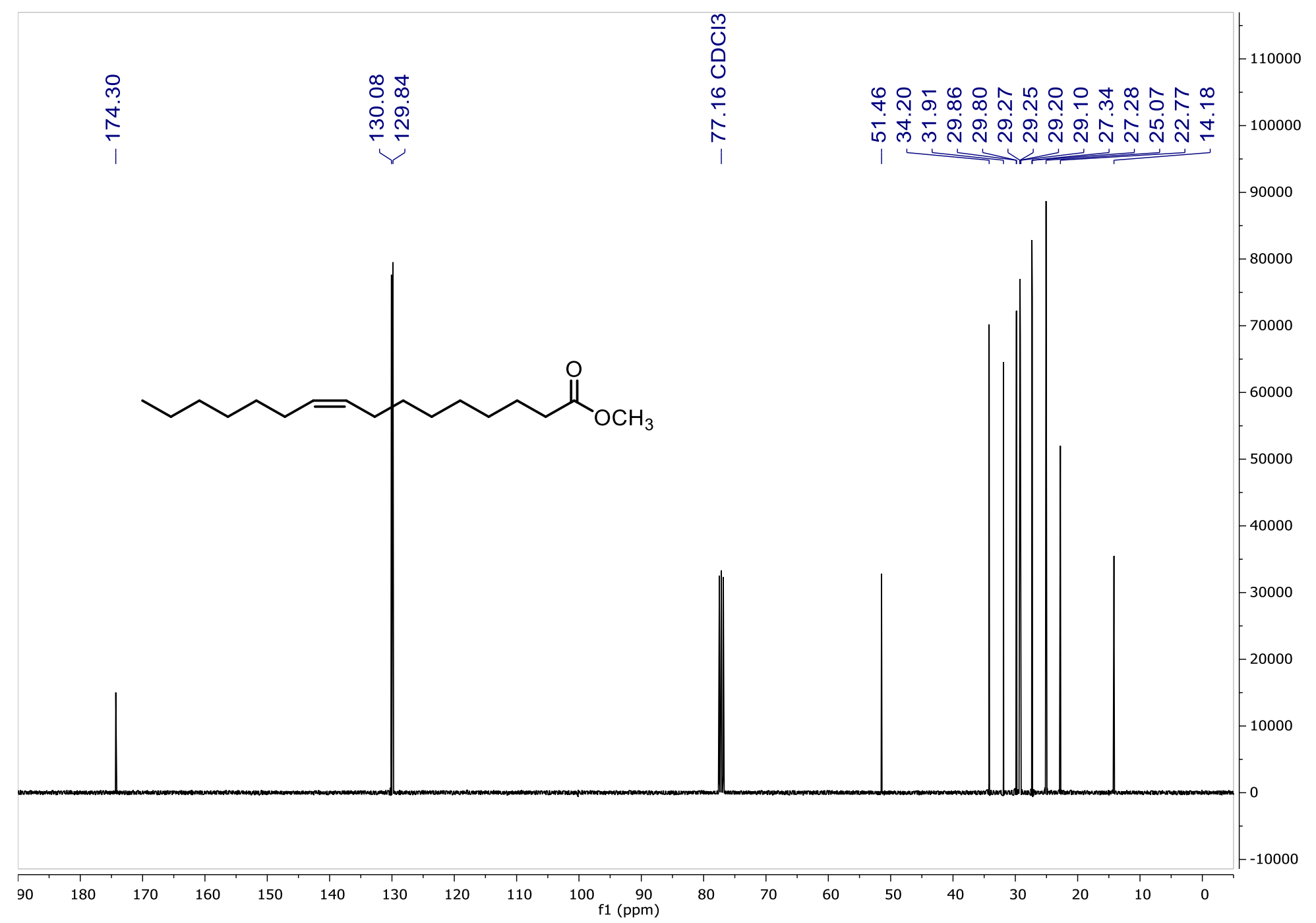


Figure S106: ${ }^{1} \mathrm{H}$ NMR $\left(400 \mathrm{MHz}, \mathrm{CDCl}_{3}\right)$ of $( \pm)$-methyl cis-9,10-metylenehexadecanoate.

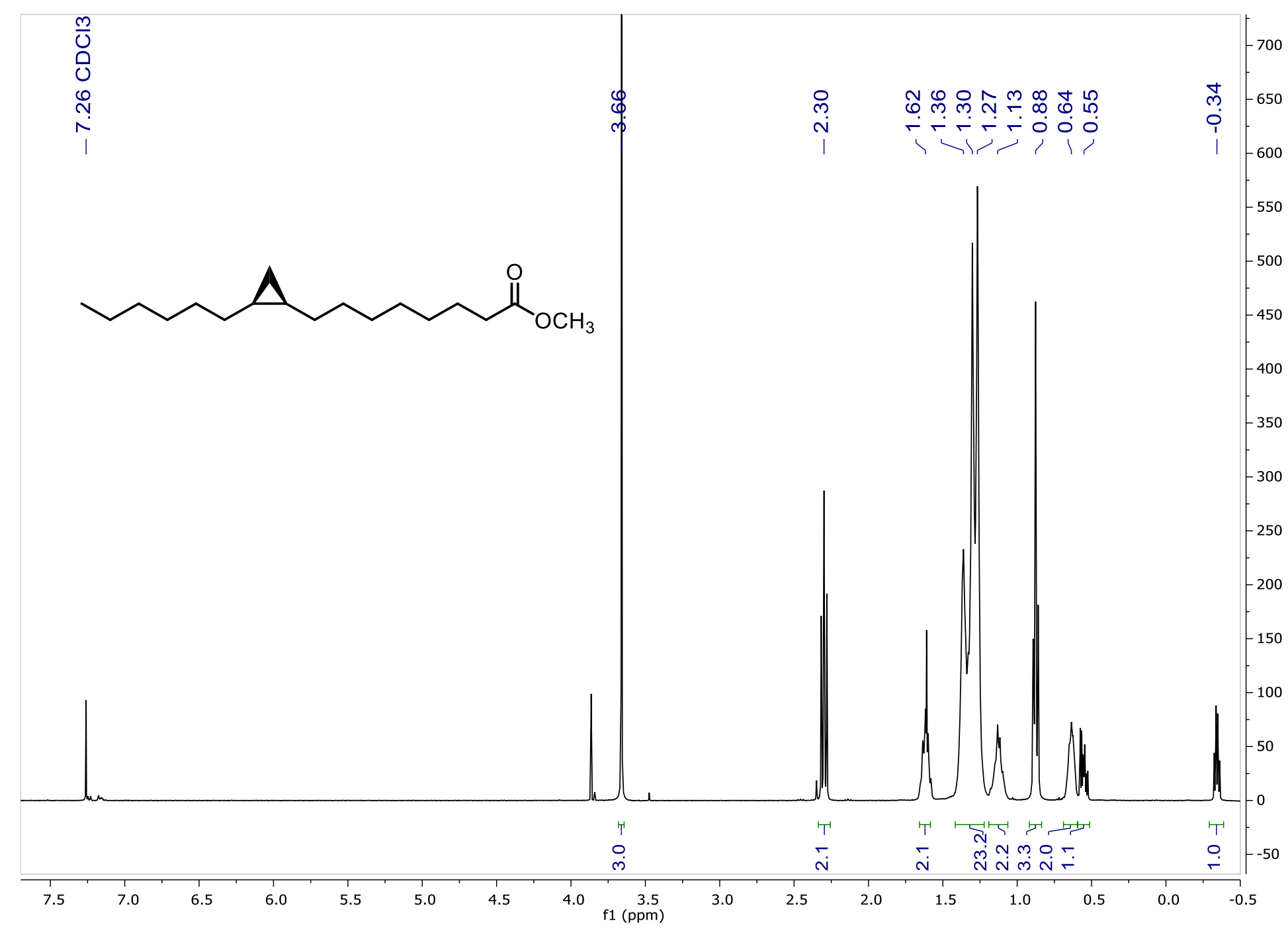


Figure S107: ${ }^{13} \mathrm{C}$ NMR (100 MHz, $\left.\mathrm{CDCl}_{3}\right)$ of $( \pm)$-methyl cis-9,10-metylenehexadecanoate.

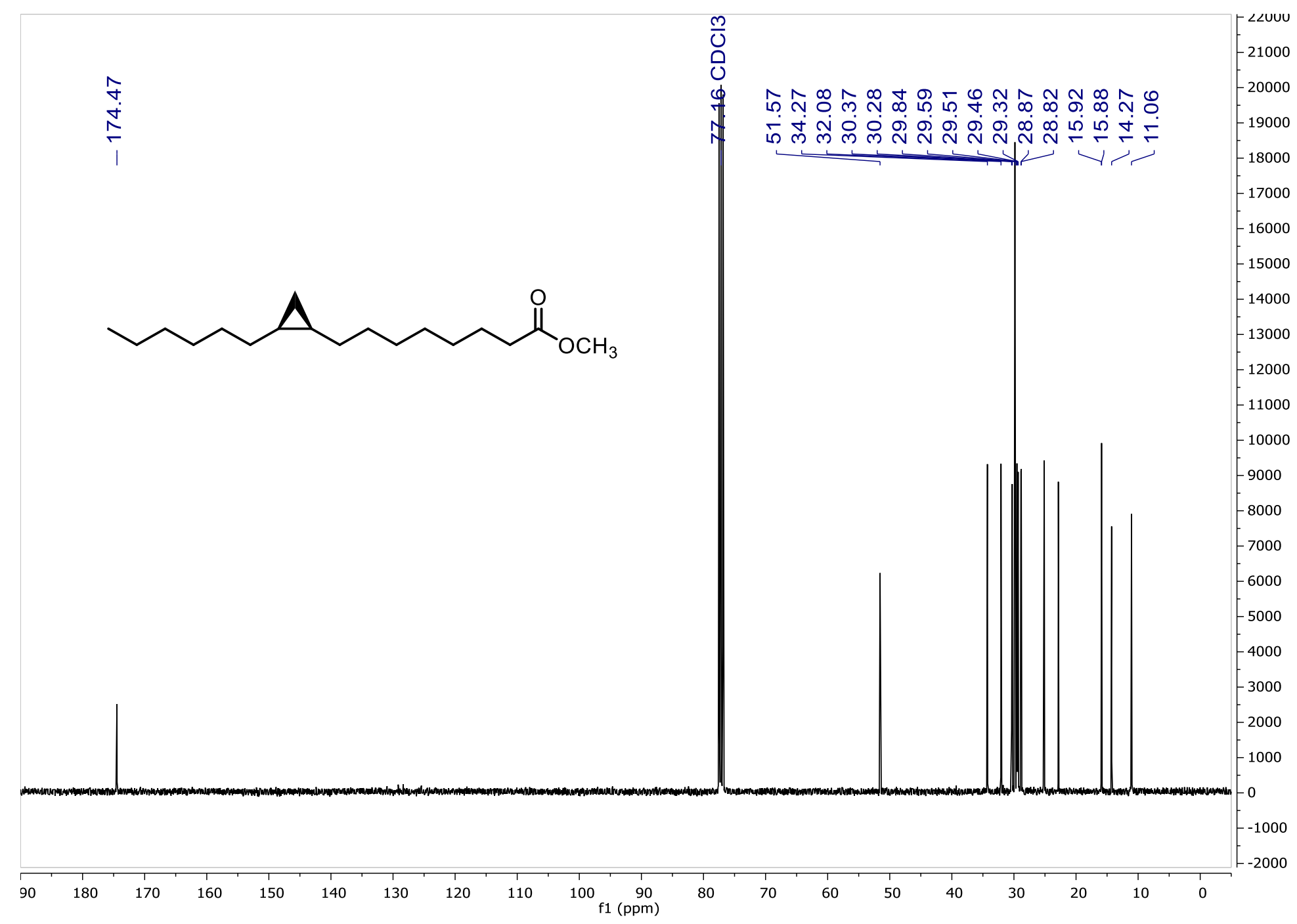


Figure S108: ${ }^{1} \mathrm{H}$ NMR $\left(400 \mathrm{MHz}, \mathrm{CDCl}_{3}\right)$ of $( \pm)$-cis-9,10-metylenehexadecanoic acid $(25, c y C 17)$.

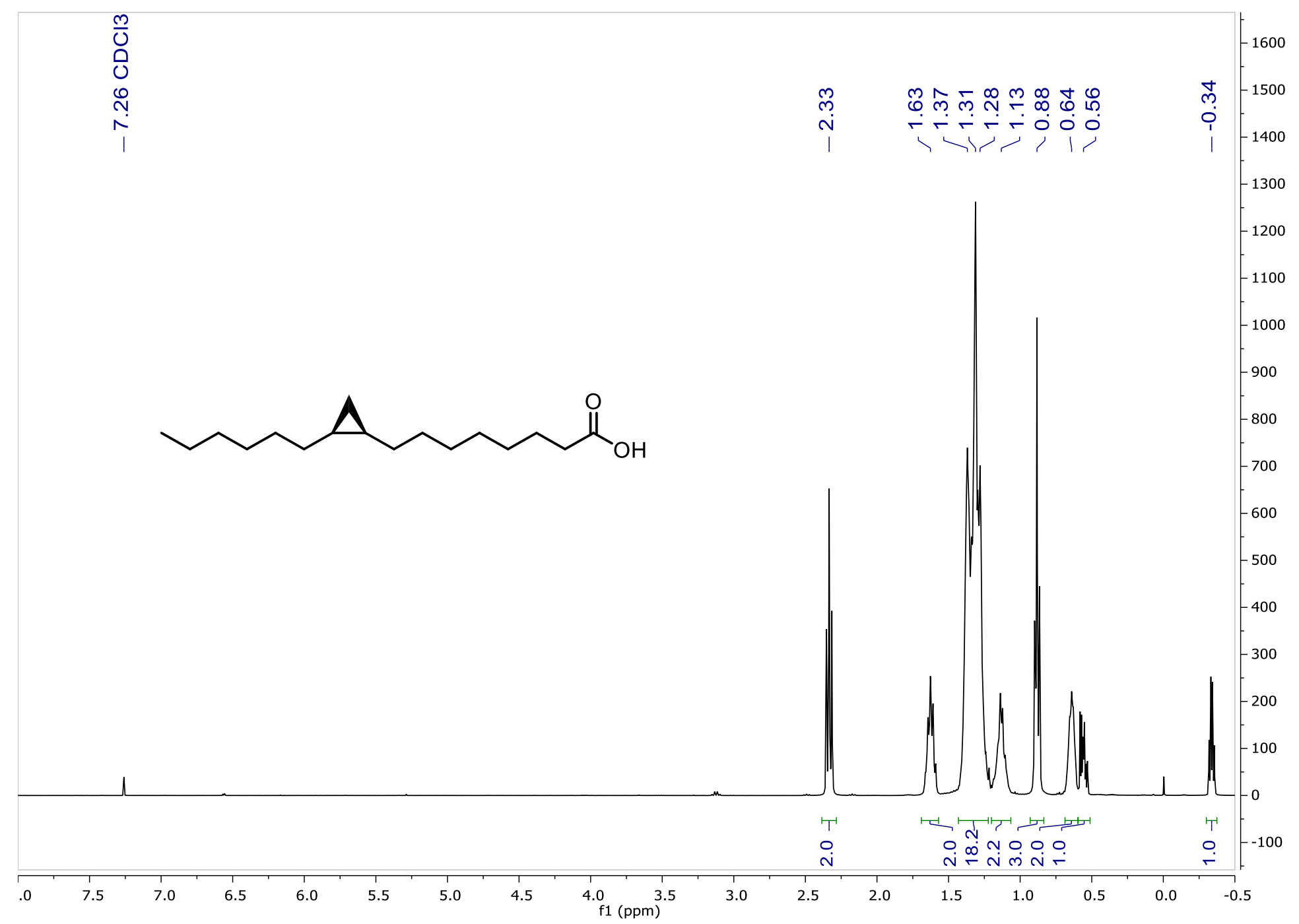


Figure S109: ${ }^{13} \mathrm{C}$ NMR $\left(100 \mathrm{MHz}, \mathrm{CDCl}_{3}\right)$ of $( \pm)$-cis-9,10-metylenehexadecanoic acid $(25, \mathrm{cyC} 17)$.

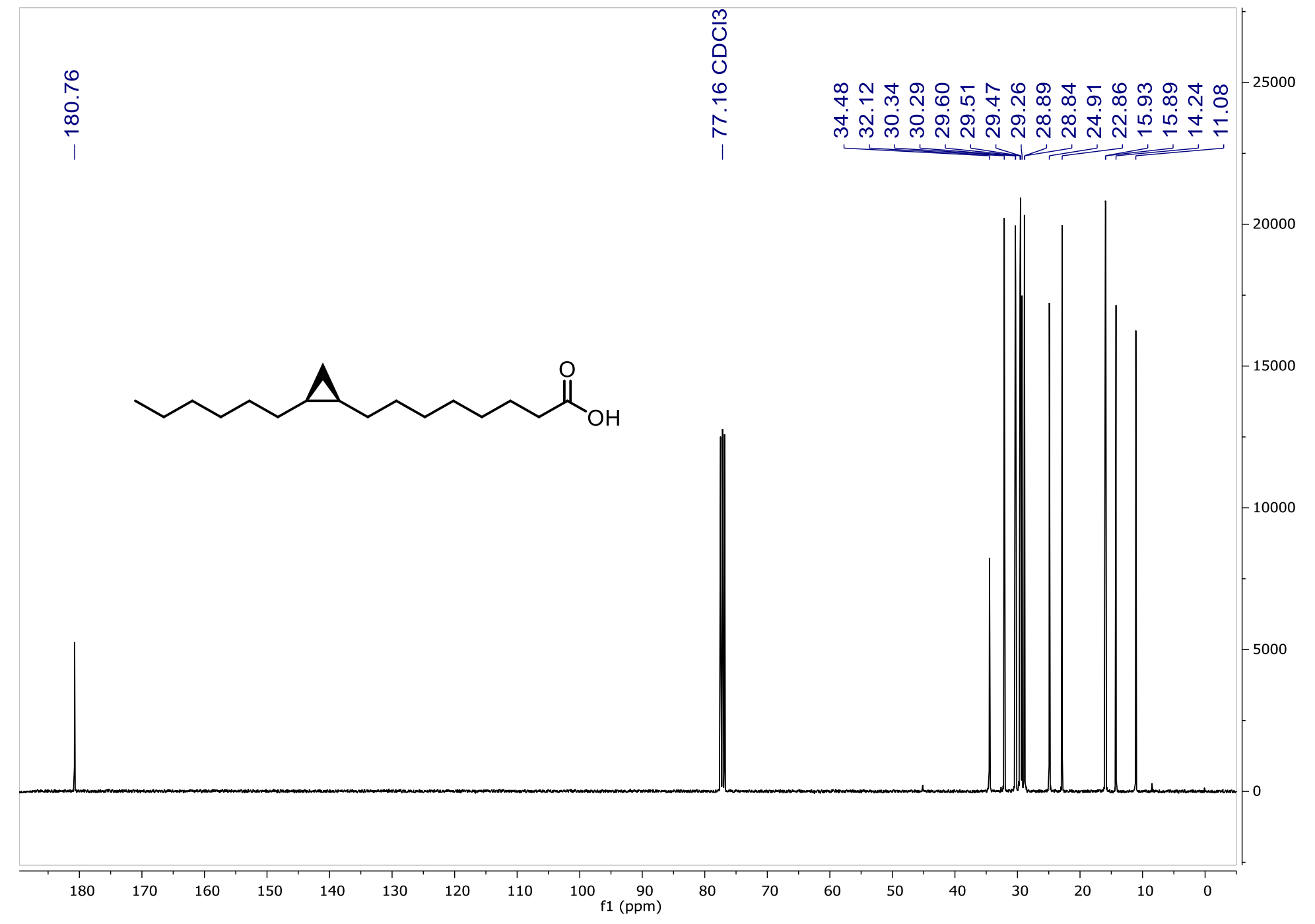


Figure S110: ${ }^{1} \mathrm{H}$ NMR (400 MHz, $\left.\mathrm{CD}_{3} \mathrm{OD}\right)$ of natural cis-3,4-methylenedecanoic acid (26, cyC11).

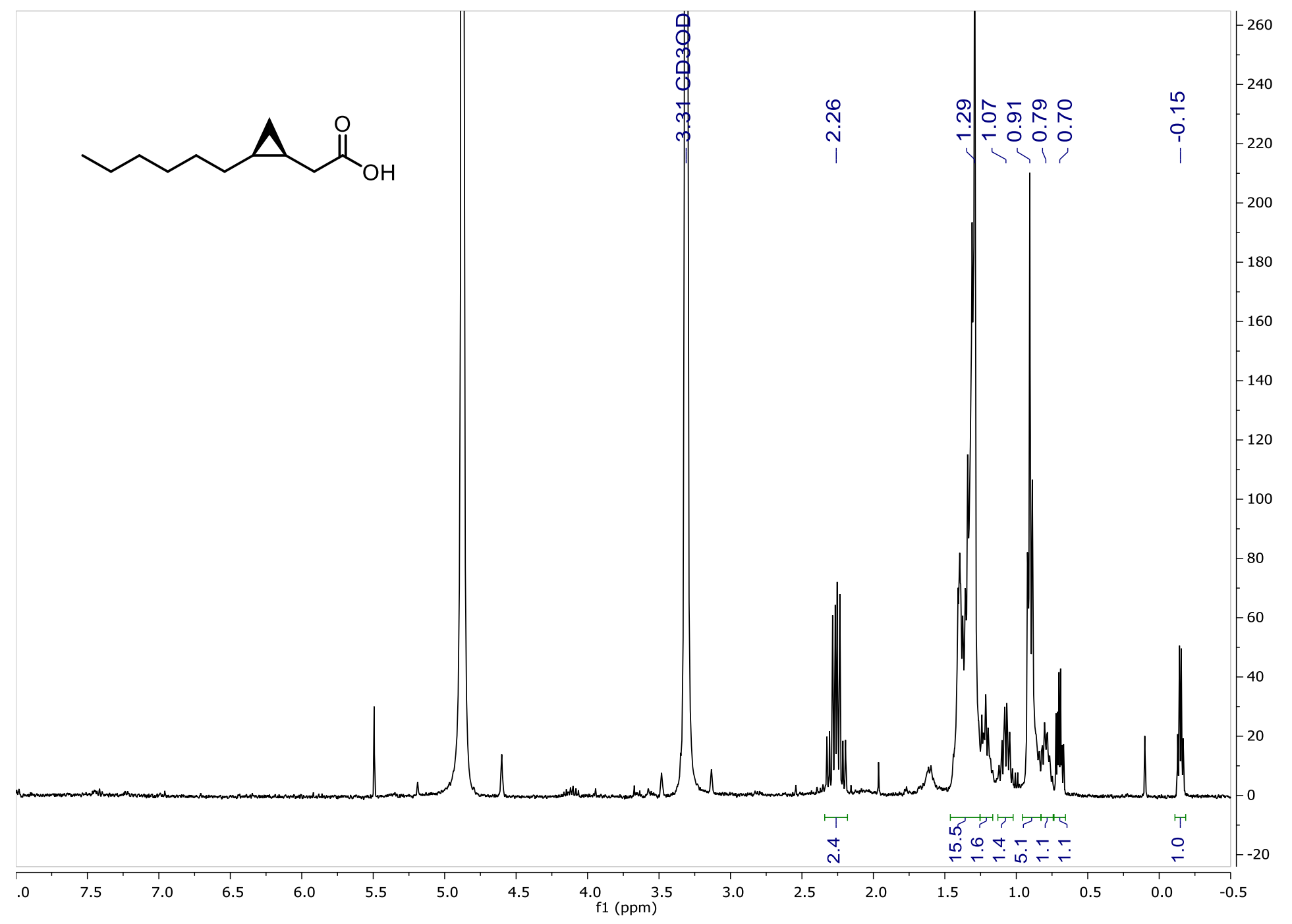


Figure S111: dqf-COSY (400 MHz, CD $\left.{ }_{3} \mathrm{OD}\right)$ of natural cis-3,4-methylenedecanoic acid (26, cyC11).

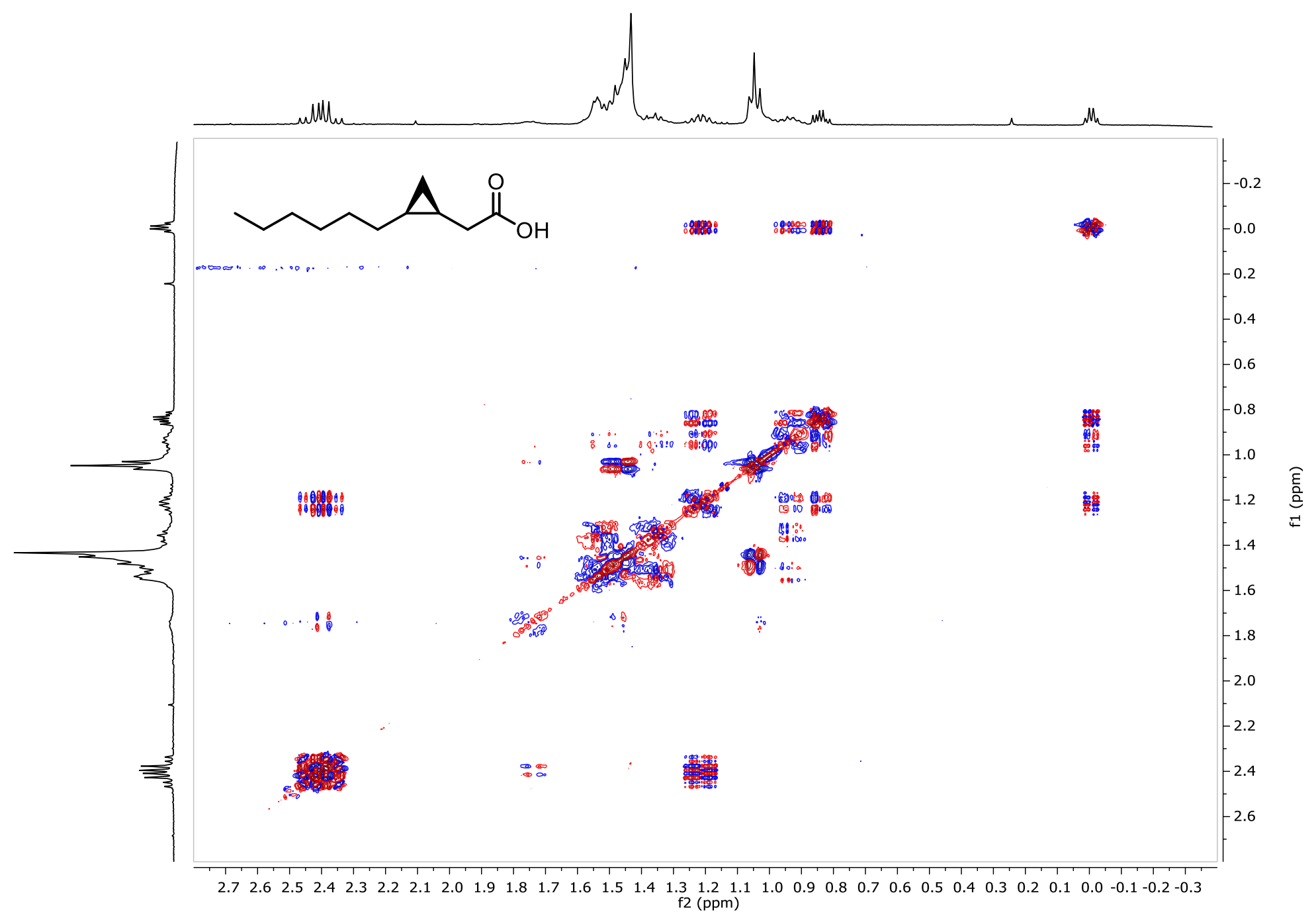


Figure S112: $\mathrm{HSQC}\left(400 \mathrm{MHz}, \mathrm{CD}_{3} \mathrm{OD}\right)$ of natural cis-3,4-methylenedecanoic acid (26, cyC11).

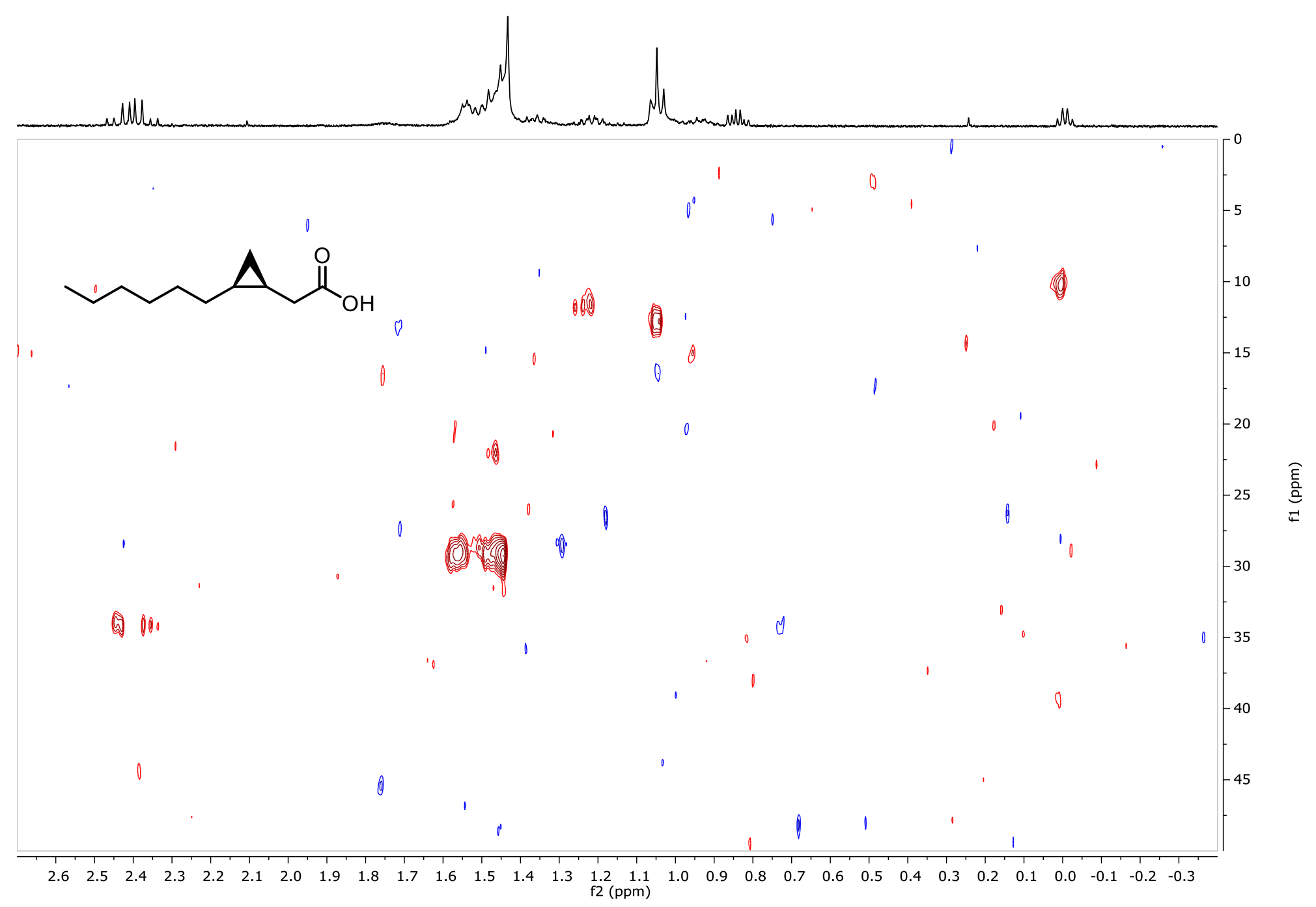


Figure S113: ${ }^{1} \mathrm{H}$ NMR (400 MHz, $\left.\mathrm{CD}_{3} \mathrm{OD}\right)$ of natural cis-3,4-methylenedecanedioic acid (27, cyC11DA).

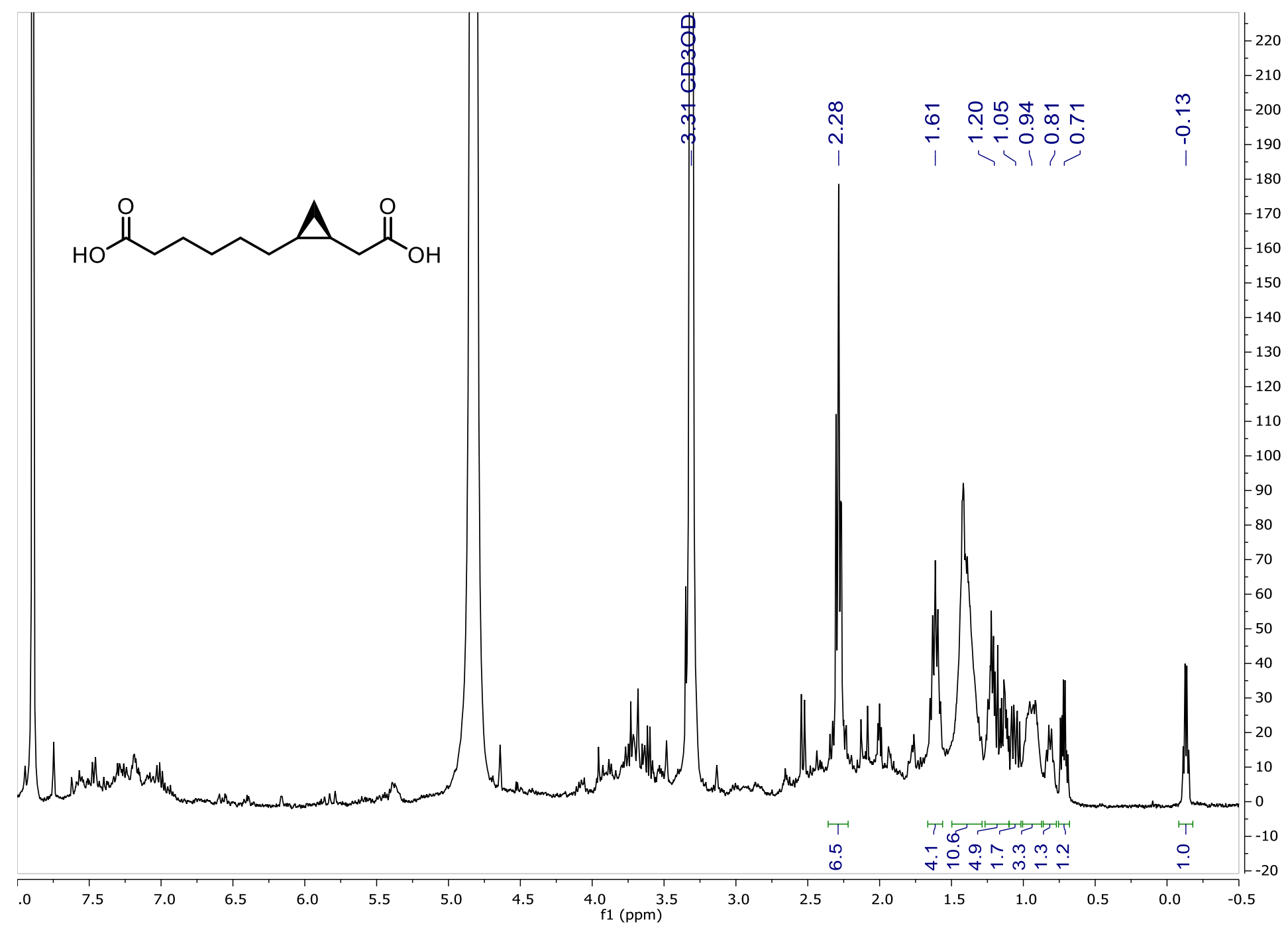


Figure S114: dqf-COSY (400 MHz, $\mathrm{CD}_{3} \mathrm{OD}$ ) of natural cis-3,4-methylenedecanedioic acid (27, cyC11DA).

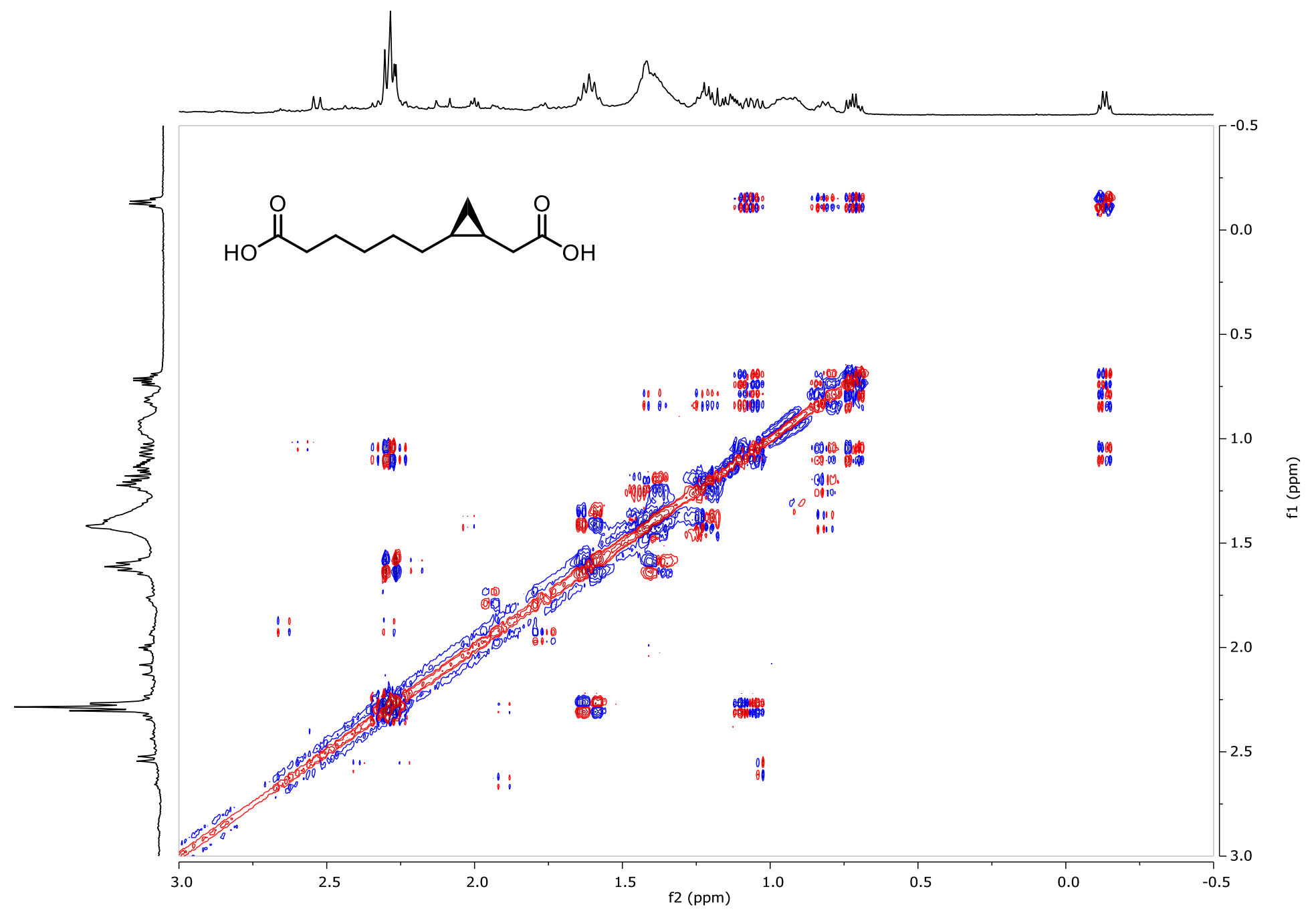


Figure S115: ${ }^{1} \mathrm{H}$ NMR $\left(400 \mathrm{MHz}, \mathrm{CD}_{3} \mathrm{OD}\right)$ of natural cis-3,4-methyleneoctanedioic acid (28, cyC9DA).

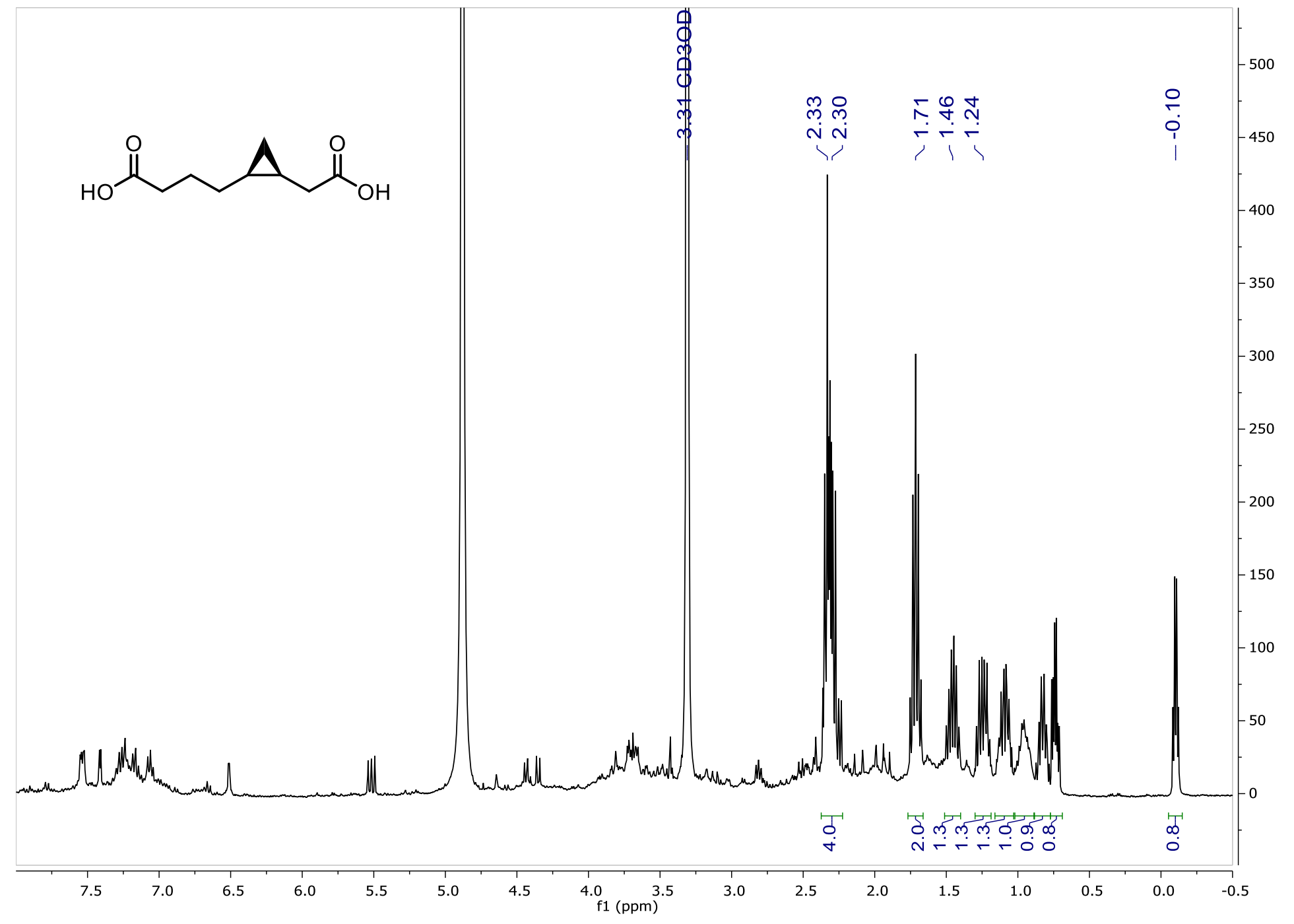


Figure S116: ${ }^{1} \mathrm{H}$ NMR (400 MHz, $\left.\mathrm{CD}_{3} \mathrm{OD}\right)$ of natural cis-3,4-methyleneoctanedioic acid (28, cyC9DA).

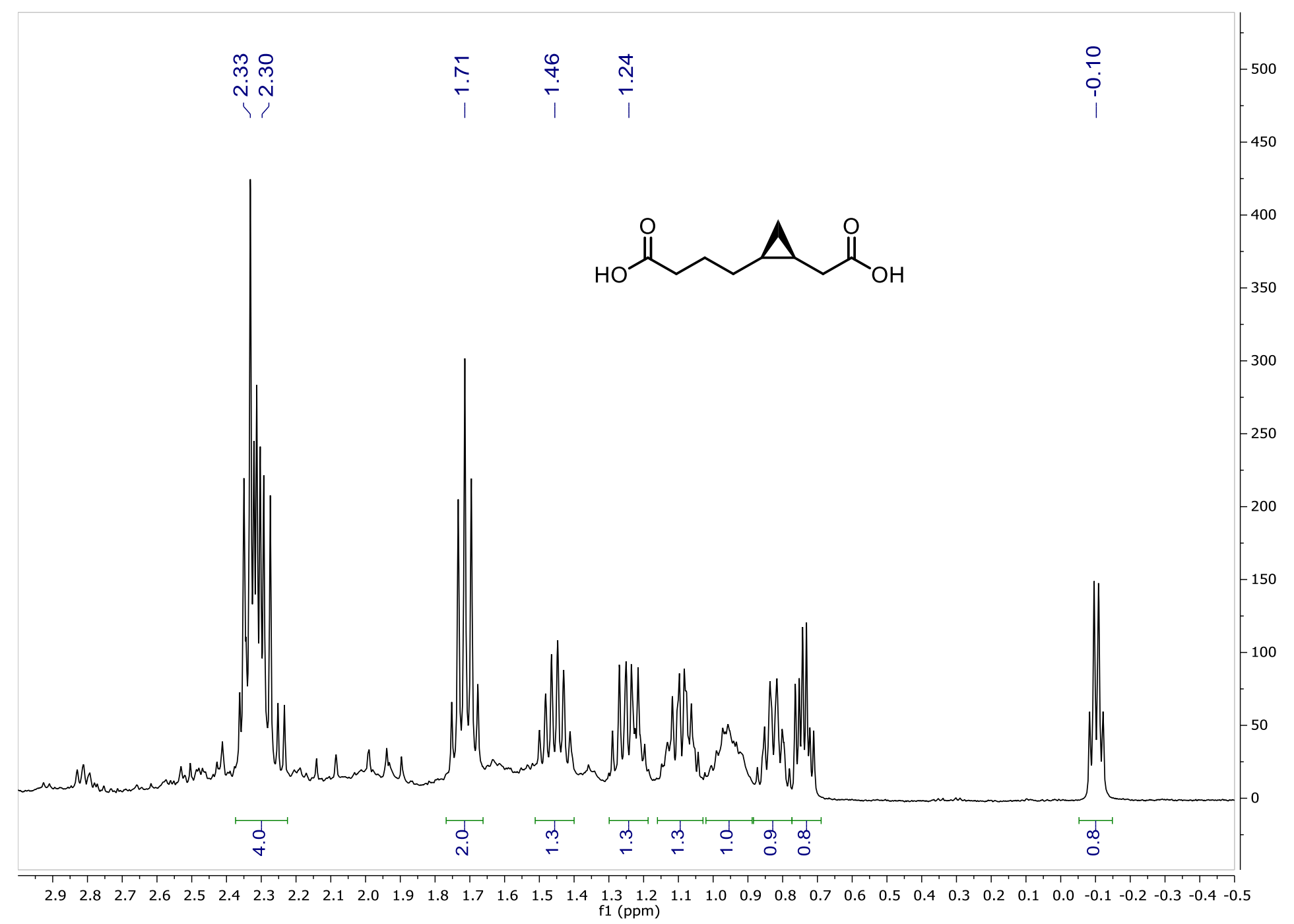


Figure S117: ${ }^{13} \mathrm{C}$ NMR (400 MHz, $\mathrm{CD}_{3} \mathrm{OD}$ ) of natural cis-3,4-methyleneoctanedioic acid (28, cyC9DA).

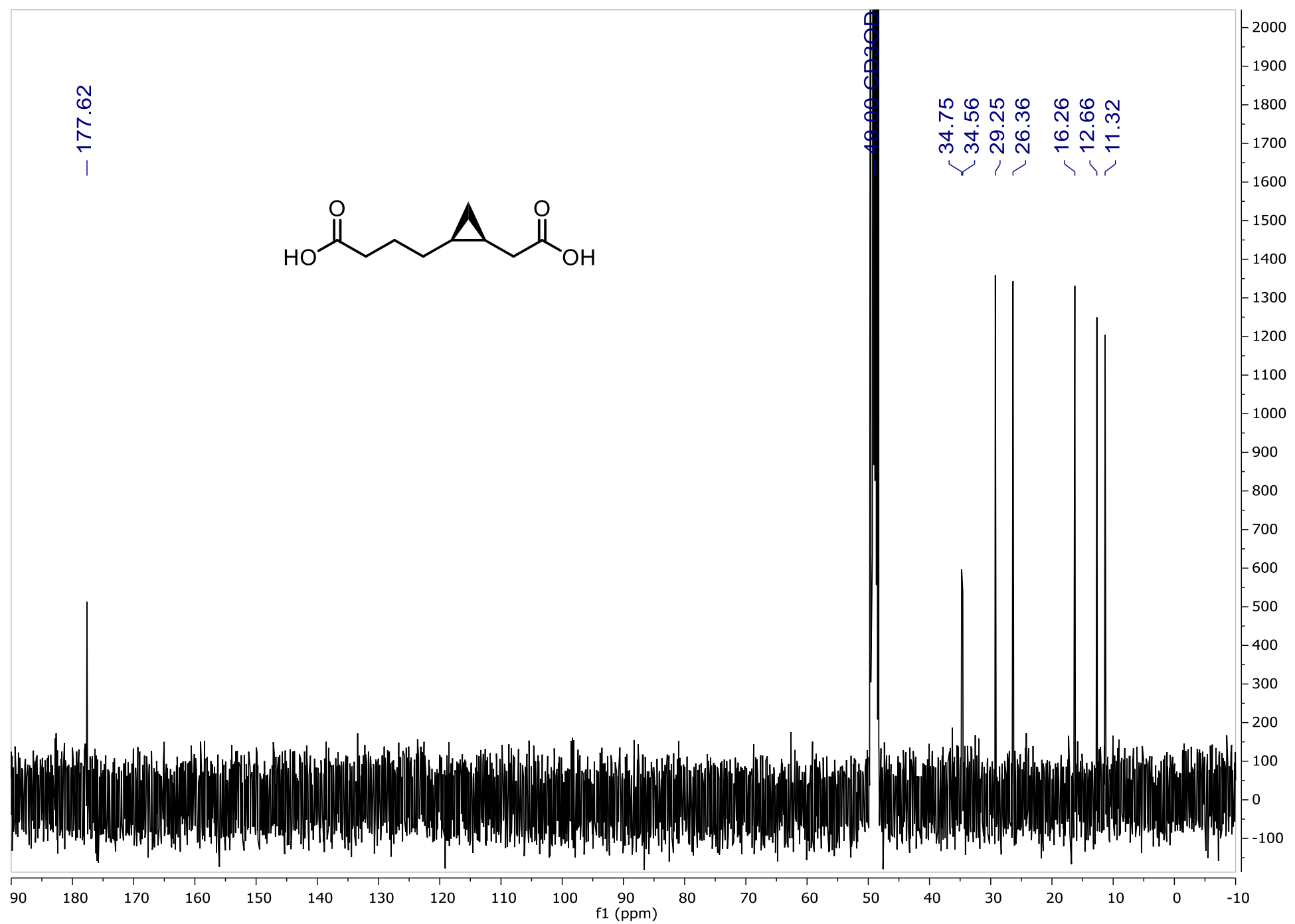


Figure S118: dqf-COSY (400 MHz, $\left.\mathrm{CD}_{3} \mathrm{OD}\right)$ of natural cis-3,4-methyleneoctanedioic acid (28, cyC9DA).

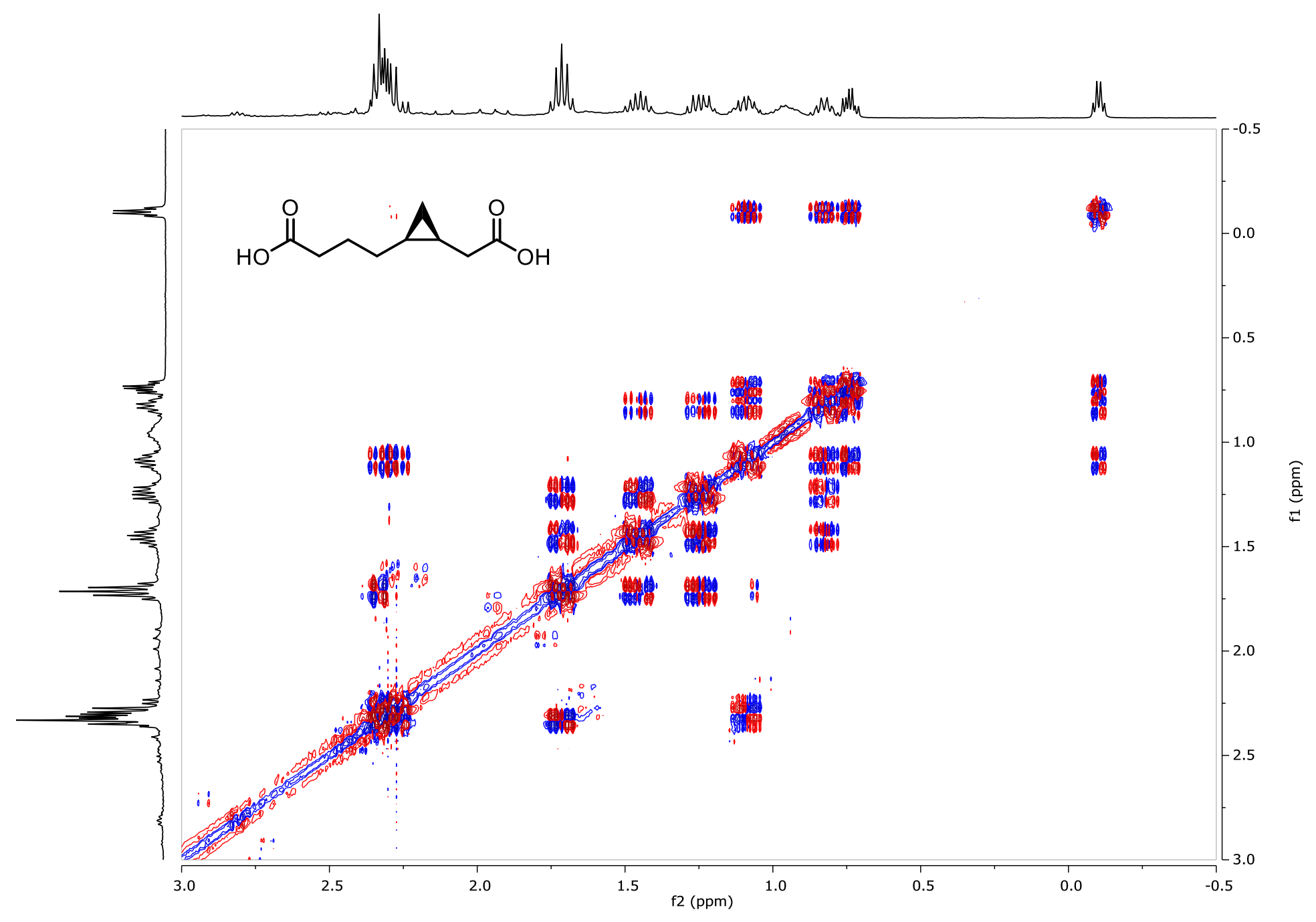


Figure S119: HSQC (400 MHz, $\left.\mathrm{CD}_{3} \mathrm{OD}\right)$ of natural cis-3,4-methyleneoctanedioic acid (28, cyC9DA).

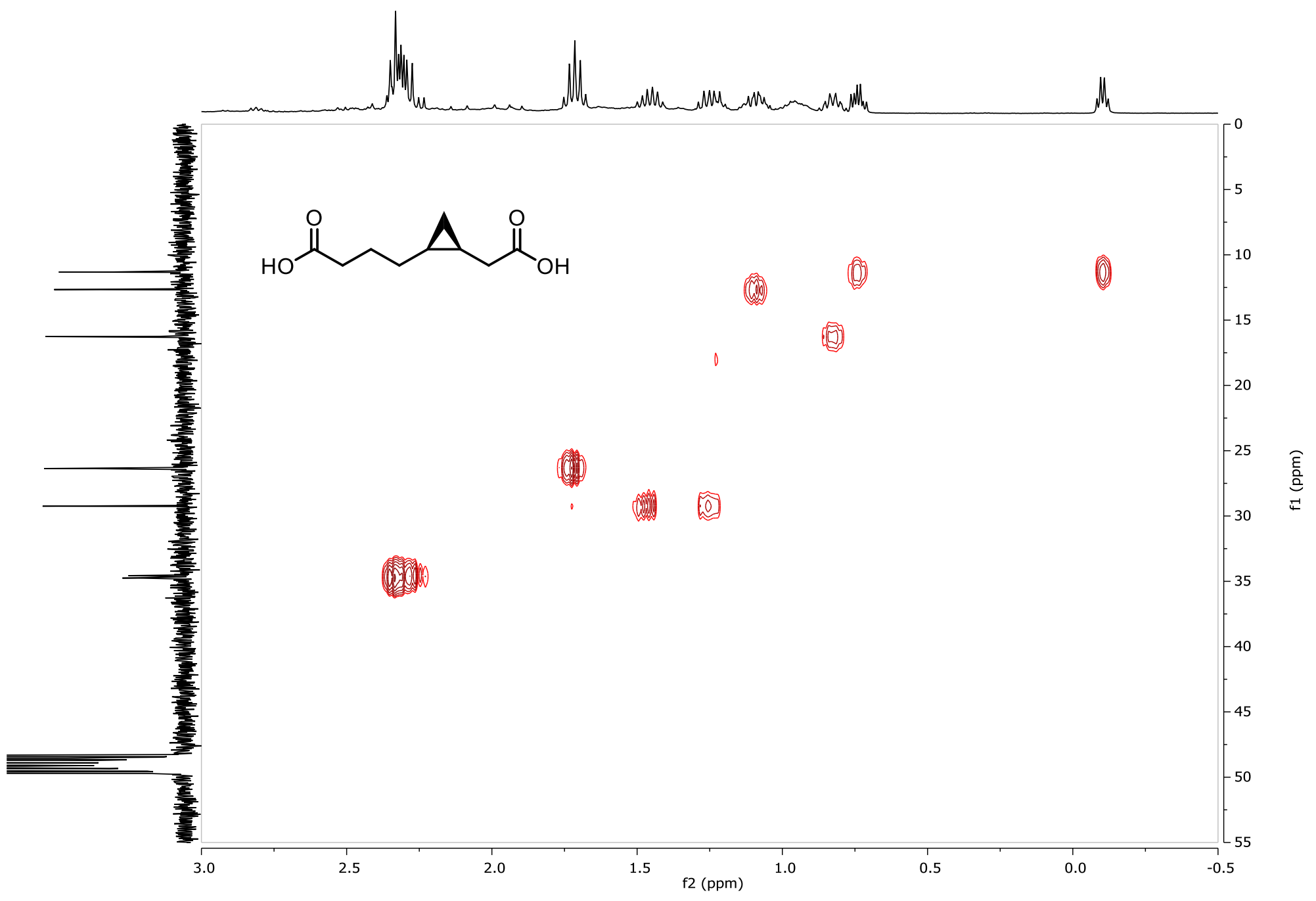


Figure S120: dqf-COSY (400 MHz, $\left.\mathrm{CD}_{3} \mathrm{OD}\right)$ of natural cis-3,4-methylenehexanedioic acid (29, cyC7DA).

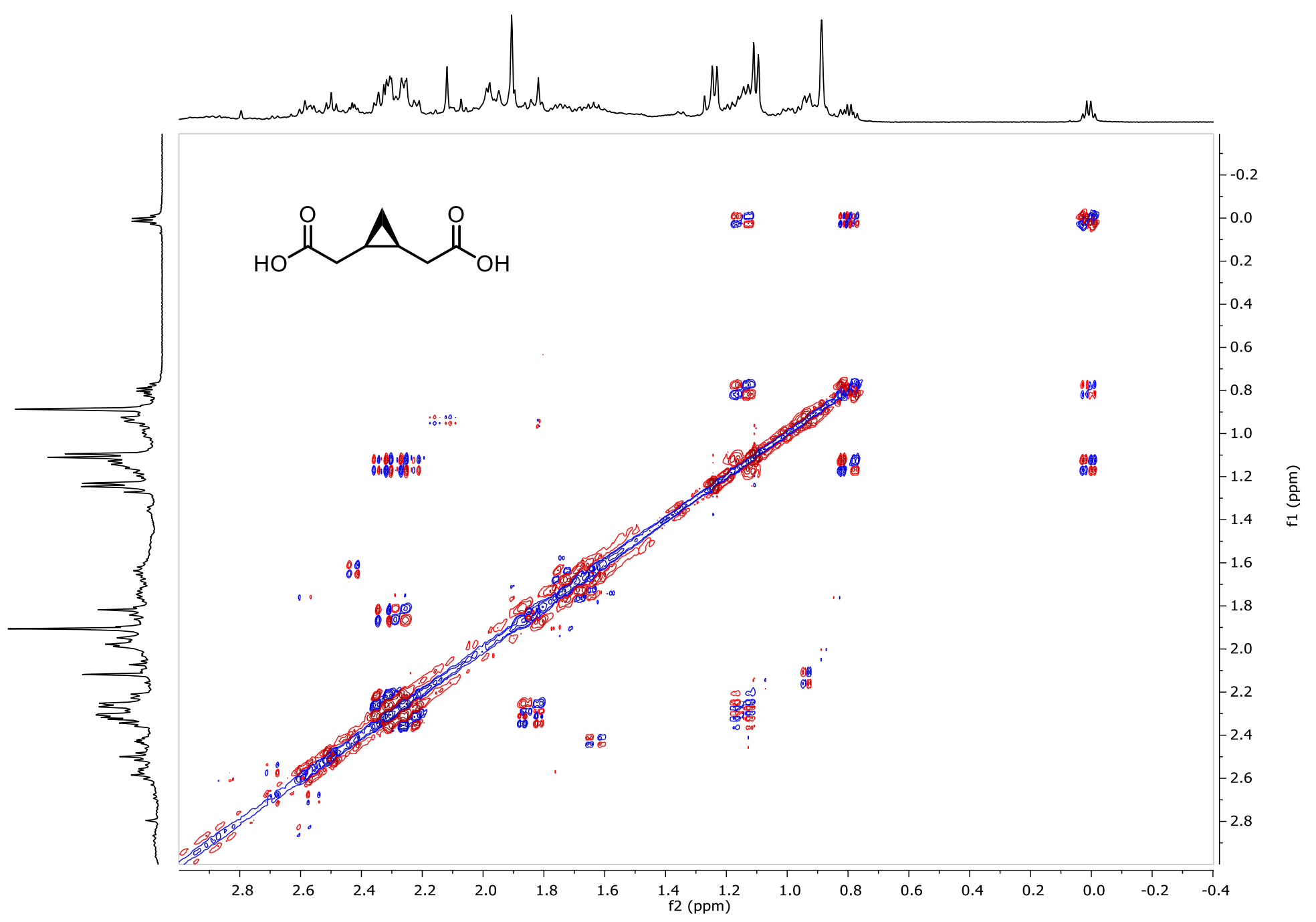


Figure S121: ${ }^{1} \mathrm{H}$ NMR $\left(400 \mathrm{MHz}, \mathrm{CD}_{3} \mathrm{OD}\right)$ of $E$. coli OP50 endometabolome extract.

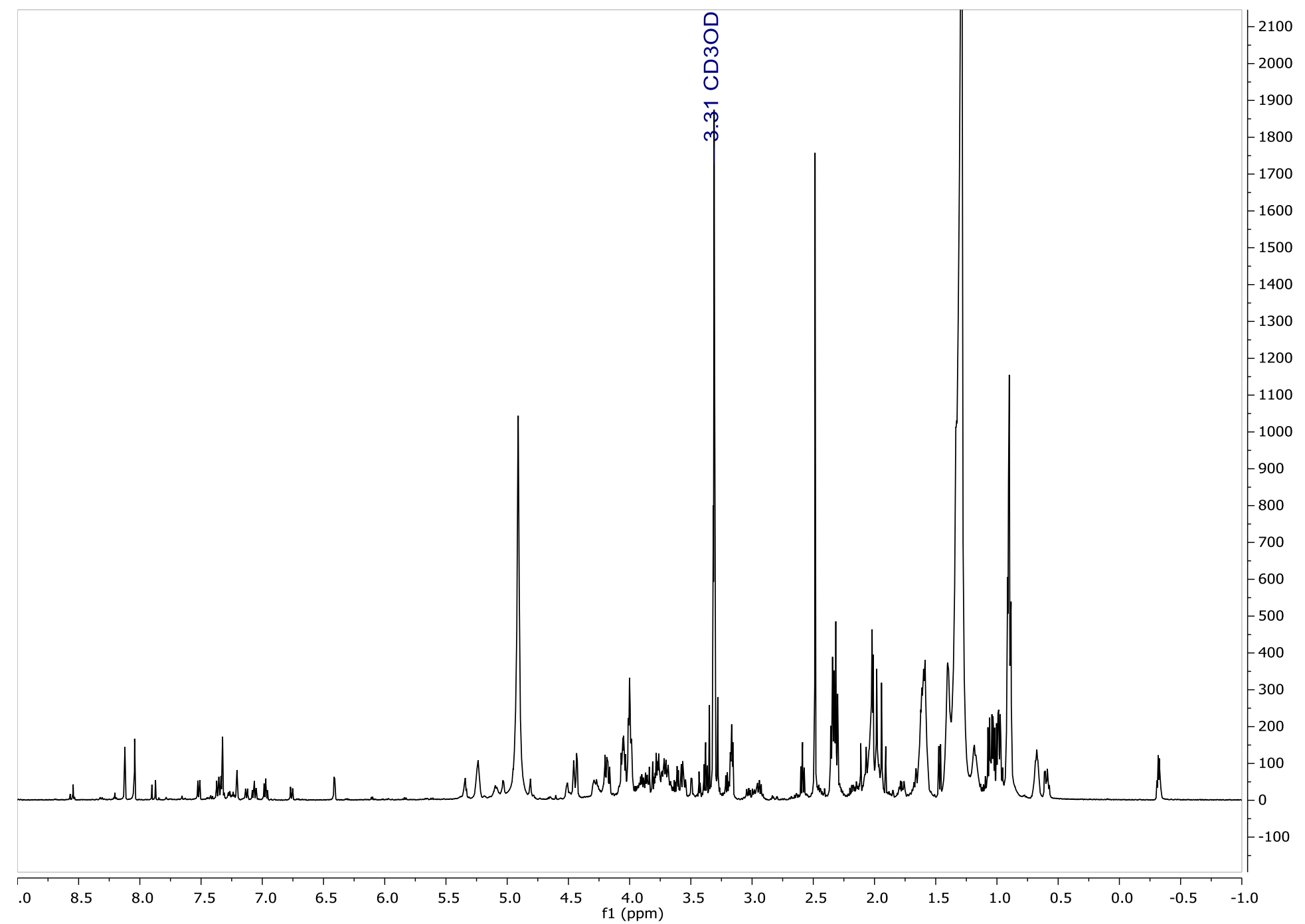


Figure S122: ${ }^{1} \mathrm{H}$ NMR $\left(400 \mathrm{MHz}, \mathrm{CD}_{3} \mathrm{OD}\right)$ of $E$. coli $\Delta c f a$ endometabolome extract.

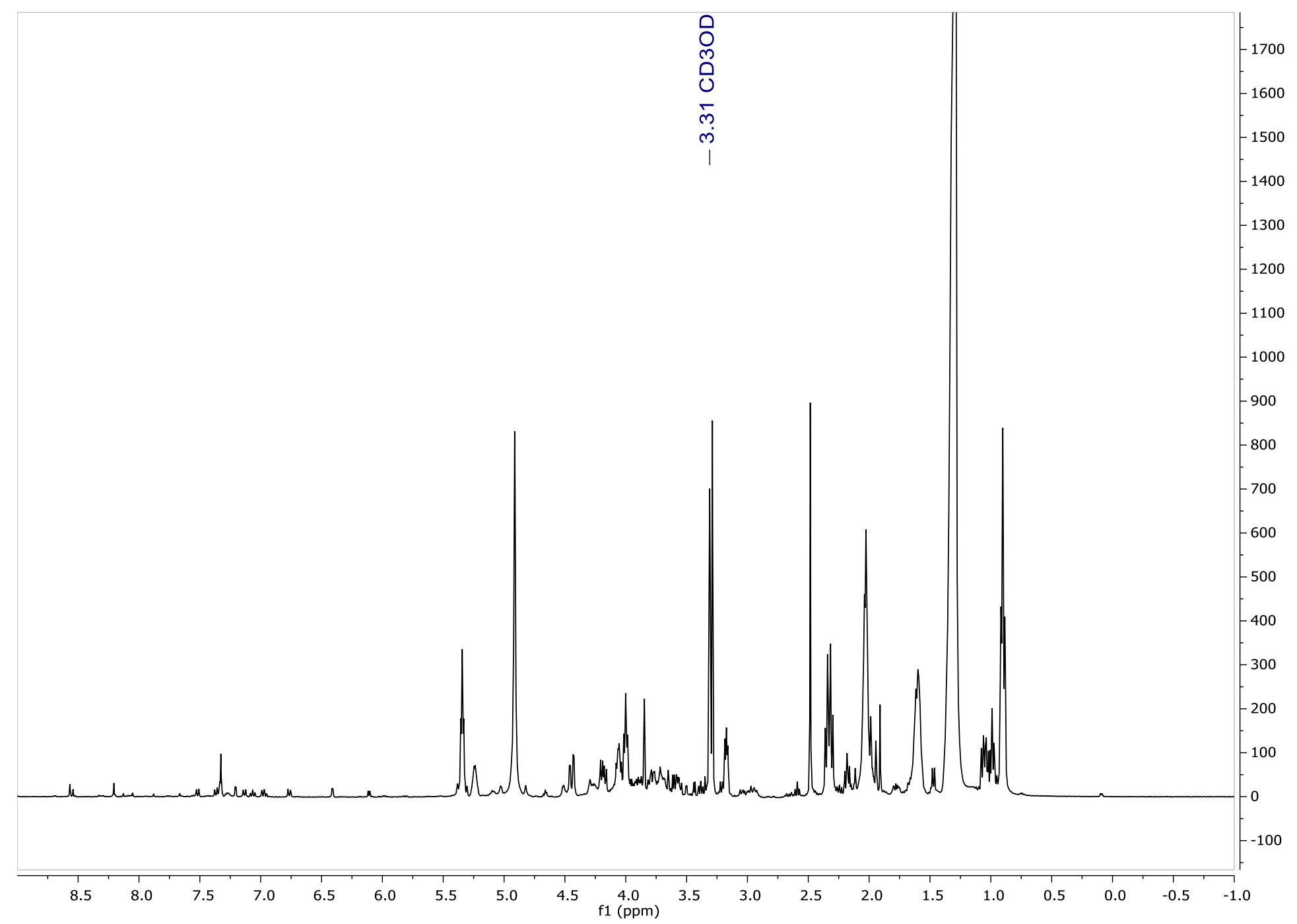


Figure S123: dqf-COSY (400 MHz, $\left.\mathrm{CD}_{3} \mathrm{OD}\right)$ of $E$. coli OP50 endometabolome extract.

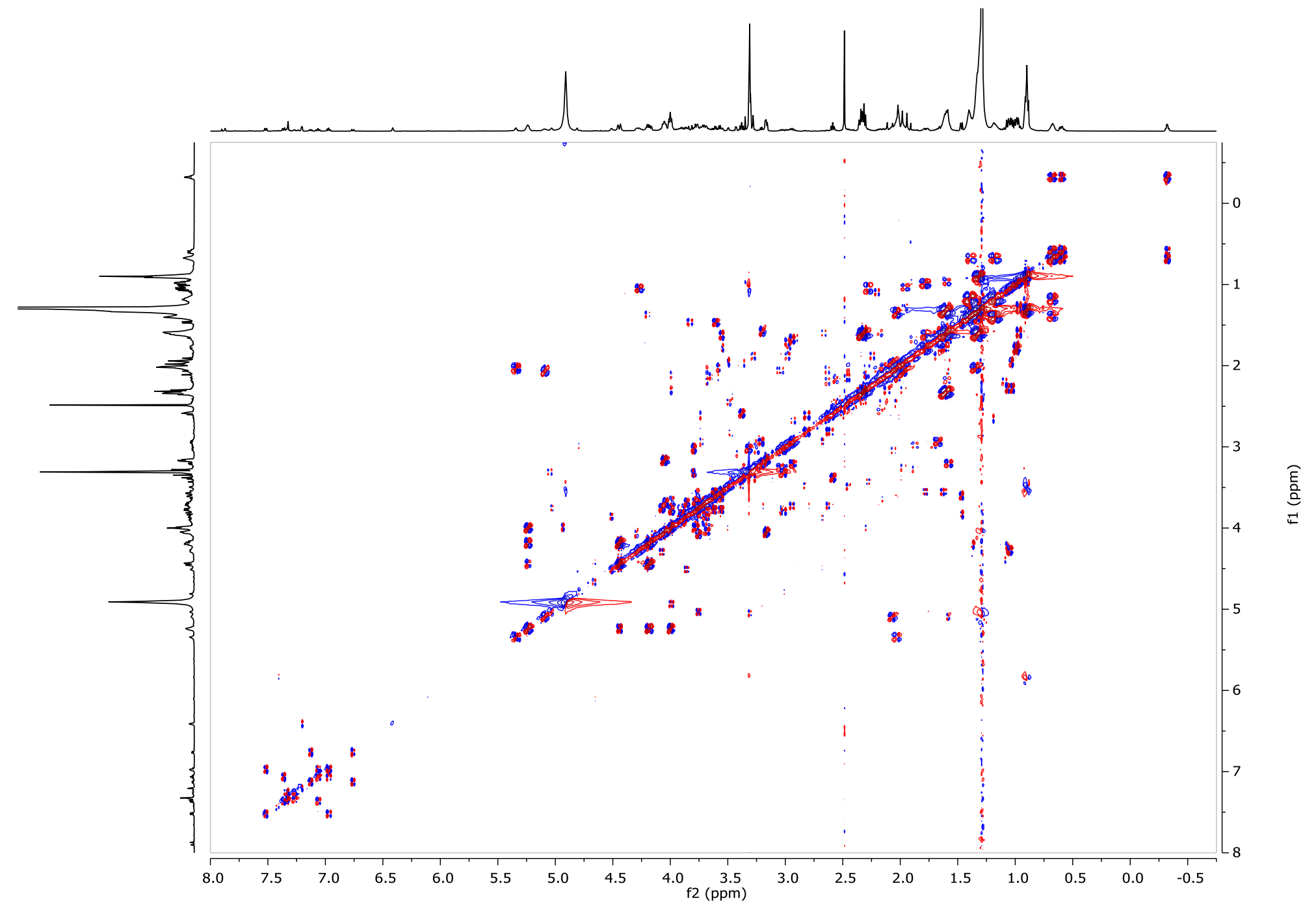


Figure S124: dqf-COSY (400 MHz, $\left.\mathrm{CD}_{3} \mathrm{OD}\right)$ of $E$. coli $\Delta c f a$ endometabolome extract.

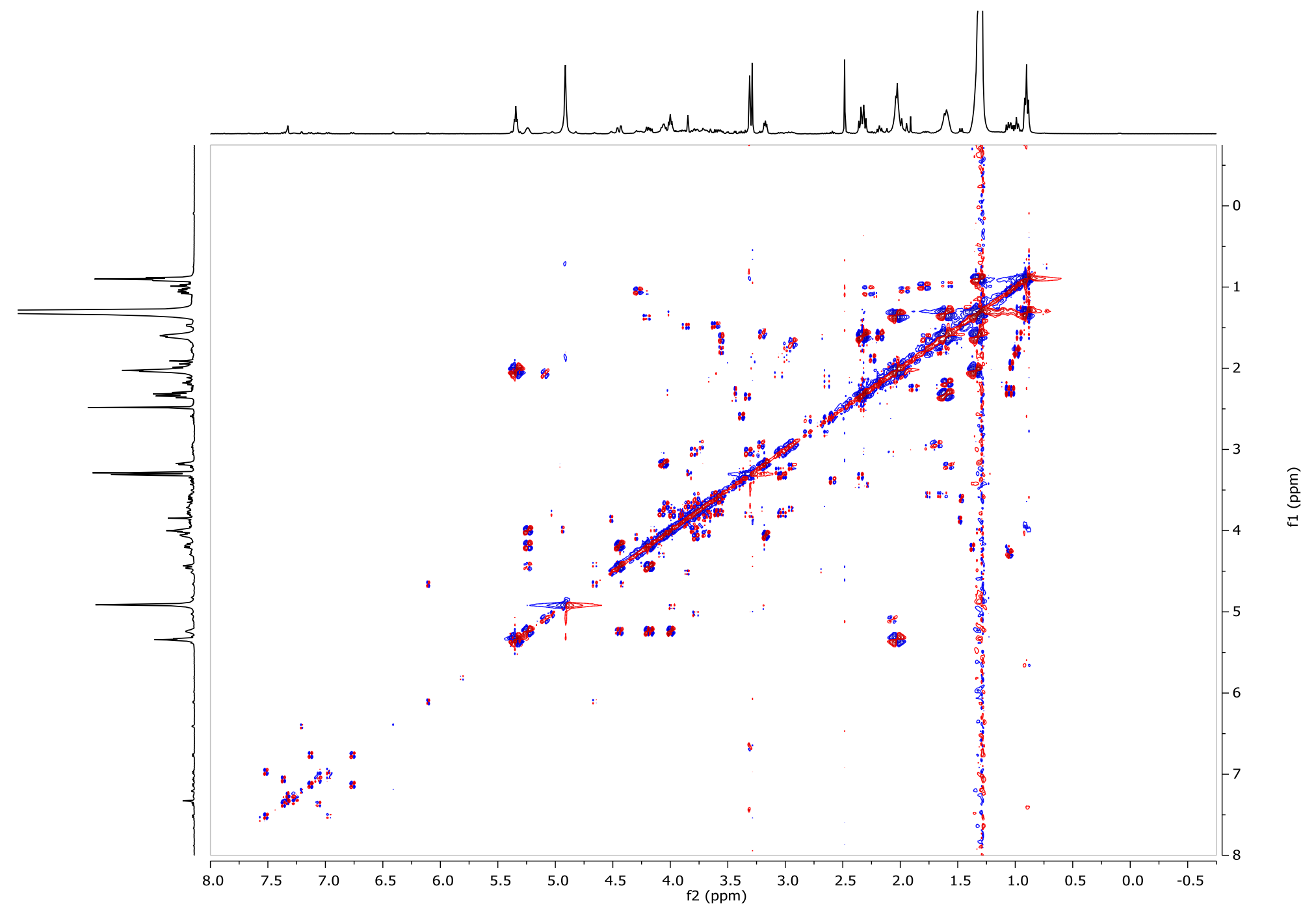




\section{References}

(1) Baba, T.; Ara, T.; Hasegawa, M.; Takai, Y.; Okumura, Y.; Baba, M.; Datsenko, K. A.; Tomita, M.; Wanner, B. L.; Mori, H. Mol. Syst. Biol. 2006, 2, 2006.0008.

(2) a) Félix, M. A. Curr. Biol. 2007, 17, 103-114; b) Diaz, S. A.; Lindström, J.; Haydon, D. T. J. Nematol. 2008, 40, 167-178; c) Diaz, S. A.; Lindström, J.; Haydon, D. T. Biol. J. Linnean Society 2010, 99, 362-369.

(3) Dey, A.; Jeon, Y; Wang, G. X.; Cutter, A. D. Genetics 2012, 191, 1257-1269.

(4) Dong, C.; Dolke, F.; von Reuss, S. H. Org. Biomol. Chem. 2016, 14, 7217-7225.

(5) Simon, J. M.; Sternberg, P. W. Proc. Natl. Acad. Sci. U. S. A. 2002, 99, 1598-1603.

(6) Yang, Z.; Lorenz, J. C.; Shi, Y. Tetrahedron Lett. 1998, 39, 8621-8624.

(7) Charette, A. B.; Juteau, H.; Lebel, H.; Molinaro, C. J. Am. Chem. Soc. 1998, 120, 11943-11952.

(8) von Reuss, S. H.; Dolke, F.; Dong, C. Anal. Chem. 2017, 89, 10570-10577.

(9) a) Kiontke, K. C.; Félix, M. A.; Ailion, M.; Rockman, M. V.; Braendle, C.; Pénigault J. B.; Fitch, D. H. A. BMC Evol. Biol. 2011, 11, 339; b) Félix, M. A.; Braendle, C.; Cutter, A. D. PLoS One 2014, 9, e94723; c) Slos, D.; Sudhaus, W.; Stevens, L.; Bert, W.; Blaxter, M. BMC Zool. 2018, 2, 4. 